\title{
Rhodium-Stabilized Diarylcarbenes Behaving as Donor/Acceptor Carbenes
}

Maizie Lee ${ }^{\ddagger}, 1$, Zhi Ren ${ }^{\ddagger 1}$, Djamaladdin G. Musaev ${ }^{*}, 2$, and Huw M. L. Davies ${ }^{*}, 1$

${ }^{1}$ Department of Chemistry, Emory University, 1515 Dickey Drive, Atlanta, Georgia 30322.

${ }^{2}$ Cherry L. Emerson Center for Scientific Computation, Emory University, 1521 Dickey Drive, Atlanta, Georgia, 30322.

E-mail: hmdavie@emory.edu

E-mail: dmusaev@emory.edu

Table of Contents

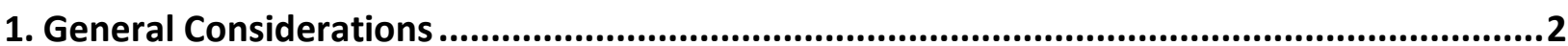

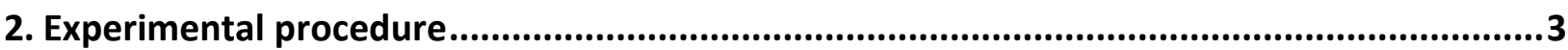

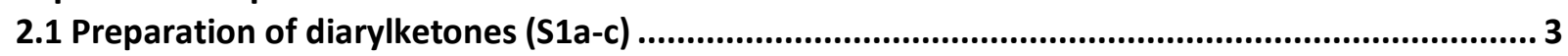

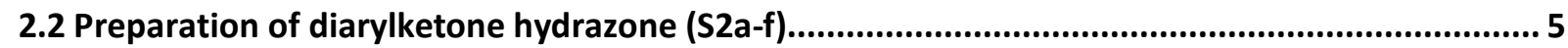

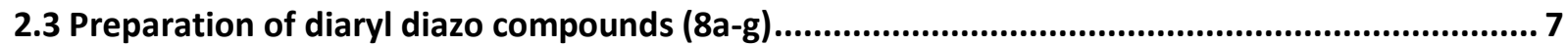

2.4 Dirhodium catalyzed cyclopropanation reaction $(11 \mathrm{a}-\mathrm{g})$............................................................. 10

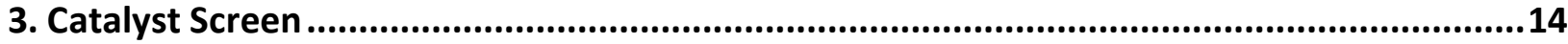

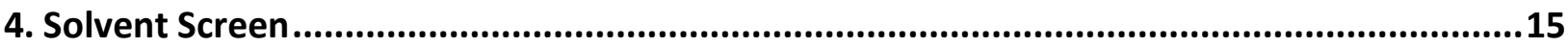

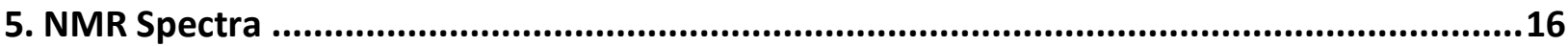

5.1 NMR of purified compounds ................................................................................ 16

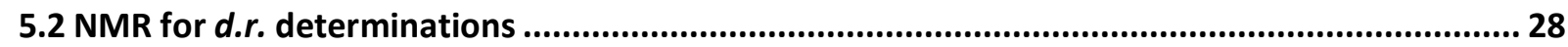

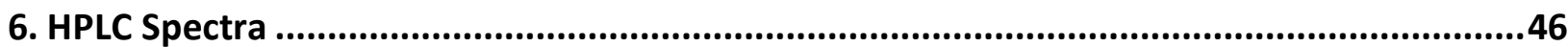

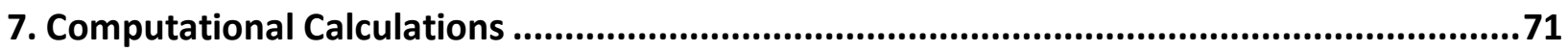

8. X-ray Crystallographic Data .............................................................................................135

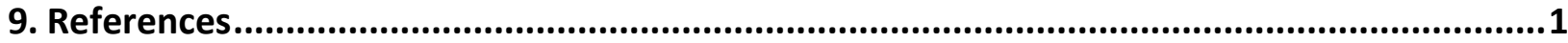




\section{General Considerations}

Substrates and reagents were purchased from the following suppliers and used without further purification: Sigma-Aldrich, Alfa-Aesar, Oakwood Chemical America, and Fisher Scientific.

All solvents were purified and dried by a Glass Contour Solvent System, and stored over $4 \AA$ molecular sieves 24 hours before use. All reactions were carried out in flamed-dried glassware unless otherwise stated. ${ }^{1} \mathrm{H}$ NMR spectra were recorded at $600 \mathrm{MHz}$ on Bruker- 600 spectrometer, Varian INOVA $500 \mathrm{MHz}$ or Varian $400 \mathrm{MHz} .{ }^{13} \mathrm{C}$ NMR spectra were recorded at $150 \mathrm{MHz}$ on Bruker-600. NMR spectra samples were prepared using deuterated chloroform $\left(\mathrm{CDCl}_{3}\right)$ with residual solvent serving as internal standard $7.26 \mathrm{ppm}$ for ${ }^{1} \mathrm{H}$ and $77.16 \mathrm{ppm}$ for ${ }^{13} \mathrm{C}$; or with Deuterated chloroform $0.03 \%$ TMS with residual TMS serving at internal standard $(0.00 \mathrm{ppm})$. Abbreviations for signal multiplicity are as follows: $\mathrm{s}=$ singlet, $\mathrm{d}=$ doublet, $\mathrm{t}=$ triplet, $\mathrm{m}=$ multiplet, $\mathrm{dd}=$ doublet of doublets $\mathrm{dt}=$ doublet of triplets. Coupling constants(J-values) were calculated directly from spectra. IR spectra were collected on a Nicolet Is10 FT-IR spectrometer. Mass spectra were taken on a Thermo Finnigan LTQ-FTMS spectrometer with APCI or NSI. Thin layer chromatographic analysis (TLC) was performed on aluminum-sheet silica gel plates, and visualized with UV light. Melting point were measured in open capillary tubes with a Mel-Temp apparatus and recorded as a range. Racemic standards for enantiomeric determination were generated with reactions with $\mathrm{Rh}_{2}(\mathrm{OAc})_{4}$ or from $\mathrm{Rh}_{2}((R) \text { and }(S) \text {-DOSP })_{4}$ which was generated by dissolving equimolar mixture of $\mathrm{R}$ and $\mathrm{S}$ catalyst in a minimal amount of benzene and lyophilizing. High performance liquid chromatography analysis (HPLC) was performed on Agilent 1100 Technologies HPLC instrument. 


\section{Experimental procedure}

\subsection{Preparation of diarylketones (S1a-c)}

\section{(4-Methoxyphenyl)(4-nitrophenyl)methanone (S1a):}

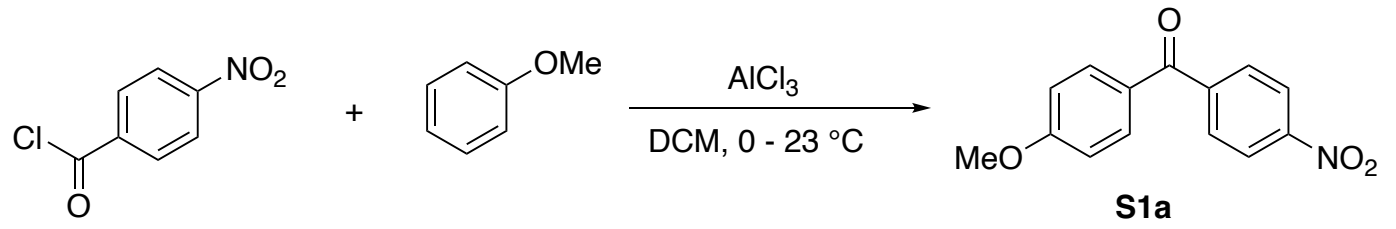

A dried 250-mL round-bottom flask was charged with anisole (15 mL, $140 \mathrm{mmol})$, 4-nitrobenzoyl chloride $(4.65 \mathrm{~g}, 25.0 \mathrm{mmol})$ in $\mathrm{CH}_{2} \mathrm{Cl}_{2}(30 \mathrm{~mL})$. The reaction mixture was cooled in an ice bath and aluminum chloride ( $3.80 \mathrm{~g}, 29 \mathrm{mmol}$ ) was slowly added. After $1 \mathrm{~h}$, the mixture was stirred under argon at $23{ }^{\circ} \mathrm{C}$ for $15 \mathrm{~h}$. The mixture was poured into ice-water $(100 \mathrm{~mL})$. Hydrochloric acid was added until emulsion disappeared $(15 \mathrm{~mL})$. The mixture was extracted with $\mathrm{CH}_{2} \mathrm{Cl}_{2}(3 \times 50$ $\mathrm{mL})$, and washed with brine $(3 \times 50 \mathrm{~mL})$. The combined organic layers were dried over $\mathrm{Na}_{2} \mathrm{SO}_{4}$ and filtered through alumina plug. The solution was concentrated in vacuum and recrystallized in hexanes to give title compound (4.8 g, $75 \%$ yield). ${ }^{1} \mathrm{H}$ NMR $\left(400 \mathrm{MHz}, \mathrm{CDCl}_{3}\right) \delta 8.32(\mathrm{~d}, 2 \mathrm{H})$, $7.89(\mathrm{~d}, J=8.7 \mathrm{~Hz}, 2 \mathrm{H}), 7.82(\mathrm{~d}, J=8.9 \mathrm{~Hz}, 2 \mathrm{H}), 7.00(\mathrm{~d}, J=8.9 \mathrm{~Hz}, 2 \mathrm{H}), 3.91(\mathrm{~s}, 3 \mathrm{H})$. This is a known compound in literature. ${ }^{1}$

General procedure for diarylketone preparation (S1b-c):<smiles>[R]c1ccc(C(=O)Cl)c(Cl)c1</smiles><smiles>COc1ccccc1</smiles><smiles>[R]c1ccc(C(=O)c2ccc([R])cc2Cl)cc1</smiles>

Procedure adopted from literature ${ }^{2}$. A $30 \mathrm{~mL}$ microwave vial was charged with desired benzoyl chloride (4.5 mmol), aluminum chloride $(1.82 \mathrm{~g}, 13.6 \mathrm{mmol})$ in $\mathrm{CH}_{2} \mathrm{Cl}_{2}(8 \mathrm{~mL})$. The mixture was irradiated in the microwave for $5 \mathrm{~min}$ at $30^{\circ} \mathrm{C}$. Benzene $(8 \mathrm{~mL}, 90 \mathrm{mmol})$ or anisole $(10 \mathrm{~mL}, 90$ mmol) was then added and irradiated for $15 \mathrm{~min}$ at $70{ }^{\circ} \mathrm{C}$. Reaction was cooled to $23^{\circ} \mathrm{C}$, and ice was then slowly added. The mixture was extracted with $\mathrm{CH}_{2} \mathrm{Cl}_{2}(3 \times 25 \mathrm{~mL})$ and washed with brine $(3 \times 25 \mathrm{~mL})$. The organic layer was dried over $\mathrm{Na}_{2} \mathrm{SO}_{4}$ and filtered through alumina and concentrated in vacuum. The product was recrystallized in hexanes to afford the corresponding desired product.<smiles>O=C(c1ccccc1)c1ccc([N+](=O)[O-])cc1Cl</smiles>

S1b

\section{(2-chloro-4-nitrophenyl)(phenyl)methanone (S1b)}

Prepared using general procedure, 2-chloro-4-nitrobenzyol chloride (1.00 g, $4.5 \mathrm{mmol})$ and benzene $(8 \mathrm{~mL}, 90 \mathrm{mmol})$ were used to afford the titled ketone $(1.01 \mathrm{~g}, 84 \%$ yield $)$, which was carried forward crude. ${ }^{1} \mathrm{H}$ NMR $\left(400 \mathrm{MHz}, \mathrm{CDCl}_{3}\right) \delta 8.36(\mathrm{~d}, J=2.1, \mathrm{~Hz}, 1 \mathrm{H}), 8.25(\mathrm{dd}, J=8.4$, $2.1 \mathrm{~Hz}, 1 \mathrm{H}), 7.81-7.76(\mathrm{~m}, 2 \mathrm{H}), 7.69-7.63(\mathrm{~m}, 1 \mathrm{H}), 7.59-7.48(\mathrm{~m}, 3 \mathrm{H})$. This is a known compound in literature. ${ }^{2}$ 
<smiles>COc1ccc(C(=O)c2ccccc2Cl)cc1</smiles>

(2-chlorophenyl)(4-methoxyphenyl)methanone (S1c)

Prepared using general procedure, 2-chlorobenzyol chloride $(0.57 \mathrm{~mL}, 4.5 \mathrm{mmol})$ and anisole $(10$ $\mathrm{mL}, 90 \mathrm{mmol})$ were used to afford the titled ketone $(0.86 \mathrm{~g}, 76 \%$ yield $) .{ }^{1} \mathrm{H}$ NMR $(400 \mathrm{MHz}$, $\left.\mathrm{CDCl}_{3}\right) \delta 7.80(\mathrm{~d}, 2 \mathrm{H}), 7.45-7.39(\mathrm{~m}, 2 \mathrm{H}), 7.38-7.34(\mathrm{~m}, 2 \mathrm{H}), 6.95(\mathrm{~d}, 2 \mathrm{H}), 3.88(\mathrm{~s}, 3 \mathrm{H})$. This is a known compound in literature. ${ }^{3}$ 


\subsection{Preparation of diarylketone hydrazone (S2a-f)}<smiles></smiles>

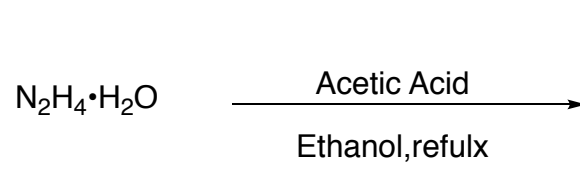<smiles></smiles>

Procedure adopted from literature. ${ }^{4}$ Hydrazine monohydrate $(8.5 \mathrm{~mL}, 88 \mathrm{mmol})$ was added to the corresponding diarylketone $(8.8 \mathrm{mmol})$ in ethanol $(100 \mathrm{~mL})$. Glacial acetic acid $(0.5 \mathrm{~mL})$ was added dropwise and mixture was heated at reflux for $20 \mathrm{~h}$. Reaction was monitored by TLC until completion. After cooling reaction was concentrated with toluene to remove excess water. The crude material was then recrystallized in ethanol to afford desired product.<smiles>N=C(c1ccccc1)c1ccc([N+](=O)[O-])cc1</smiles>

\section{((4-nitrophenyl)(phenyl)methylene)hydrazone (S2a)}

Prepared using general procedure, (4-Nitrophenyl)(phenyl)methanone $(2.0 \mathrm{~g}, 8.8 \mathrm{mmol})$ was used to afford the titled hydrazone (1.60g, 75\% yield). ${ }^{1} \mathrm{H}$ NMR $\left(400 \mathrm{MHz}, \mathrm{CDCl}_{3}\right) \delta 8.10(\mathrm{~d}, J=9.0$ $\mathrm{Hz}, 2 \mathrm{H}), 7.60-7.47(\mathrm{~m}, 5 \mathrm{H}), 7.27-7.19(\mathrm{~m}, 2 \mathrm{H}), 5.75(\mathrm{~s}, 2 \mathrm{H})$. This is a known compound in literature. $^{5}$<smiles>COc1ccc(C(=N)c2ccccc2)cc1</smiles>

\section{((4-methoxyphenyl)(phenyl)methylene)hydrazone (S2b)}

Prepared using general procedure, 4-methoxybenzophenone $(1.87 \mathrm{~g}, 8.8 \mathrm{mmol})$ was used to afford the titled hydrazone $(1.42 \mathrm{~g}, 71 \%$ yield $) .{ }^{1} \mathrm{H}$ NMR $\left(400 \mathrm{MHz}, \mathrm{CDCl}_{3}\right) \delta 7.53-7.50(\mathrm{~m}, 2 \mathrm{H}), 7.48$ $-7.41(\mathrm{~m}, 1 \mathrm{H}), 7.38(\mathrm{~d}, J=9.06 \mathrm{~Hz}, 1 \mathrm{H}) 7.31-7.27(\mathrm{~m}, 2 \mathrm{H}), 6.81(\mathrm{~d}, J=9.07 \mathrm{~Hz}, 2 \mathrm{H})$.), 5.31 (s, $2 \mathrm{H}), 3.80(\mathrm{~s}, 3 \mathrm{H})$. This is a known compound in literature. ${ }^{4,6}$<smiles>COc1ccc(C(=N)c2ccc([N+](=O)[O-])cc2)cc1</smiles>

\section{((4-methoxyphenyl)(4-nitrophenyl)methylene)hydrazone (S2c)}

Prepared using general procedure, (4-methoxyphenyl)(4-nitrophenyl)methanone (2.30 g, 8.8 mmol) was used to afford the titled hydrazone (1.63 g, 68\% yield). ${ }^{1} \mathrm{H} \mathrm{NMR}\left(600 \mathrm{MHz}, \mathrm{CDCl}_{3}\right) \delta$ $8.13(\mathrm{~d}, J=9.10 \mathrm{~Hz}, 2 \mathrm{H}), 7.61(\mathrm{~d}, J=9.15 \mathrm{~Hz}, 2 \mathrm{H}), 7.21(\mathrm{~d}, J=8.79 \mathrm{~Hz}, 2 \mathrm{H}), 7.08$ (d, $J=8.79$ $\mathrm{Hz}, 2 \mathrm{H}), 5.76$ (s, 2H), 3.89 (s, 3H). ${ }^{13} \mathrm{C} \mathrm{NMR}\left(150 \mathrm{MHz}, \mathrm{CDCl}_{3}\right) \delta 160.30,147.05,145.80,144.96$, 130.24, 126.75, 123.48, 123.41 115.21, 55.40. IR (in $\left.\mathrm{CDCl}_{3}\right)$ 3419, 3297, 1607, 1508, 1334, 1247, 854, 835. HRMS (+p APCL) calculated for $\mathrm{C}_{14} \mathrm{H}_{13} \mathrm{~N}_{3} \mathrm{O}_{3}[\mathrm{M}+\mathrm{H}]^{+} 272.1030$ found 272.10264. Melting point: $140-142{ }^{\circ} \mathrm{C}$. 
<smiles>N=C(c1ccccc1)c1ccccc1Cl</smiles>

S2d

((2-chlorophenyl)(phenyl)methylene)hydrazone (S2d)

Prepared using general procedure, 2-chlorobenzophenone $(1.90 \mathrm{~g}, 8.8 \mathrm{mmol})$ was used to afford the titled hydrazone $\left(1.68 \mathrm{~g}, 83 \%\right.$ yield). ${ }^{1} \mathrm{H}$ NMR $\left(400 \mathrm{MHz}, \mathrm{CDCl}_{3}\right) \delta 7.61-7.54(\mathrm{~m}, 1 \mathrm{H}), 7.48$ $-7.42(\mathrm{~m}, 4 \mathrm{H}), 7.35-7.28(\mathrm{~m}, 3 \mathrm{H}), 7.25-7.22(\mathrm{~m}, 1 \mathrm{H}), 5.40(\mathrm{~s}, 2 \mathrm{H}) .^{7}$<smiles>N=C(c1ccccc1)c1ccc([N+](=O)[O-])cc1Cl</smiles>

\section{$\mathrm{S} 2 \mathrm{e}$}

(2-chloro-4-nitrophenyl)(phenyl)methylene)hydrazone (S2e)

Prepared using general procedure, (2-chloro-4-nitrophenyl)(phenyl)methanone (2.30 g, $8.8 \mathrm{mmol})$ was used to afford the titled hydrazone $\left(1.00 \mathrm{~g}, 41 \%\right.$ yield). ${ }^{1} \mathrm{H}$ NMR $\left(600 \mathrm{MHz}, \mathrm{CDCl}_{3}\right) \delta 8.46$ $(\mathrm{d}, J=2.2 \mathrm{~Hz}, 1 \mathrm{H}), 8.30(\mathrm{dd}, J=8.3,2.2 \mathrm{~Hz}, 1 \mathrm{H}), 7.48(\mathrm{dd}, J=8.3,0.4 \mathrm{~Hz}, 1 \mathrm{H}), 7.40-7.37(\mathrm{~m}$, $2 \mathrm{H}), 7.34-7.31(\mathrm{~m}, 3 \mathrm{H}), 5.42(\mathrm{~s}, 2 \mathrm{H}) .{ }^{13} \mathrm{C} \mathrm{NMR}\left(150 \mathrm{MHz}, \mathrm{CDCl}_{3}\right) \delta$ 148.90, 144.10, 139.30, 136.13, 134.81, 131.73, 128.77, 128.57, 125.63, 122.85. IR(in $\mathrm{CDCl}_{3}$ ) 3413, 3300, 3098, 1519, 1352, 771, 695. HRMS (+p APCL) calculated for $\mathrm{C}_{13} \mathrm{H}_{10} \mathrm{ClN}_{3} \mathrm{O}_{2}[\mathrm{M}+\mathrm{H}]^{+} 276.0535$ found 276.05314. Melting point: $145-146^{\circ} \mathrm{C}$.<smiles>COc1ccc(C(=N)c2ccccc2Cl)cc1</smiles>

\section{S2f}

((2-chlorophenyl)(4-methoxyphenyl)methylene)hydrazone (S2f)

Prepared using general procedure, (2-chlorophenyl)(4-methoxyphenyl)methanone (2.20 g, 8.8 $\mathrm{mmol})$ was used to afford the titled hydrazone $(1.50 \mathrm{~g}, 65 \%$ yield $) .{ }^{1} \mathrm{H}$ NMR $\left(600 \mathrm{MHz}, \mathrm{CDCl}_{3}\right) \delta$ $7.57-7.55(\mathrm{~m}, 1 \mathrm{H}), 7.44-7.41(\mathrm{~m}, 2 \mathrm{H}), 7.38(\mathrm{~d}, \mathrm{~J}=8.90 \mathrm{~Hz}, 2 \mathrm{H}), 7.24-7.22(\mathrm{~m}, 1 \mathrm{H}), 6.84(\mathrm{~d}, J$ $=8.9 \mathrm{~Hz}, 2 \mathrm{H}), 5.27(\mathrm{~s}, 2 \mathrm{H}), 3.80(\mathrm{~s}, 3 \mathrm{H}) .{ }^{13} \mathrm{C} \mathrm{NMR}\left(150 \mathrm{MHz}, \mathrm{CDCl}_{3}\right) \delta 159.88,146.75,133.04$, 132.47, 130.48, 130.46, 130.35, 130.04, 127.86, 127.23, 113.77, 55.29.IR (in $\mathrm{CDCl}_{3}$ ) 3404, 2957, $2836,1510,1249,1166,1033,835,766,749$. HRMS (+p APCL) calculated for $\mathrm{C}_{14} \mathrm{H}_{13} \mathrm{ClN}_{2} \mathrm{O}$ $[\mathrm{M}+\mathrm{H}]^{+} 261.0789$ found 261.07852 . Melting point: $90-92{ }^{\circ} \mathrm{C}$. 


\subsection{Preparation of diaryl diazo compounds (8a-g)}<smiles>[R2]c1ccc(C(=N)c2ccc(Br)cc2)cc1</smiles><smiles>[CH]=[N+]S(=O)(=O)c1ccc(C)cc1</smiles><smiles>[R][R]1ccc(C(=[W])c2ccc(Br)cc2)cc1</smiles>

Procedure adopted from literature. ${ }^{8}$ A $50 \mathrm{~mL}$ dried round bottom flask was charged with hydrazone $(1.1 \mathrm{mmol})$ and THF $(4 \mathrm{~mL})$ followed by tsNIK $(1.2 \mathrm{mmol})$ prepared by following the literature procedure. ${ }^{4}$ The potassium hydroxide in $1 \mathrm{M}$ solution $(1 \mathrm{~mL})$ was slowly added to the flask. The reaction was monitored by TLC, with disappearance of all starting hydrazone derivatives by 1.5 $\mathrm{h}$. The reaction was poured into $5 \mathrm{~mL}$ potassium hydroxide in $1 \mathrm{M}$ solution and extracted with diethyl ether $(2 \times 30 \mathrm{~mL})$. The organic layers were combined then washed with brine $(2 \times 30 \mathrm{~mL})$ and dried over $\mathrm{MgSO}_{4}$. After removal of the solvent, the desired diazo compound was obtained. If necessary diazo was purified using an alumina column under gradient 0 to $5 \%$ diethyl ether in hexanes. Product was stored under Argon at $-20{ }^{\circ} \mathrm{C}$.<smiles>N#Cc1ccccc1</smiles>

$8 a$

\section{(Diazomethylene)dibenzene (8a)}

Prepared using general procedure, benzophenone hydrazone $(216 \mathrm{mg}, 1.10 \mathrm{mmol})$ was used to afford the titled diazo compound (200 mg, $94 \%$ yield). ${ }^{1} \mathrm{H}$ NMR $\left(500 \mathrm{MHz}, \mathrm{CDCl}_{3}\right) \delta 7.42-7.38$ $(\mathrm{m}, 2 \mathrm{H}), 7.31(\mathrm{~d}, \mathrm{~J}=7.3 \mathrm{~Hz} 2 \mathrm{H}), 7.20(\mathrm{t}, J=7.3 \mathrm{~Hz}, 1 \mathrm{H})$. This is a known compound in literature. ${ }^{8}$<smiles>N#CC(c1ccccc1)c1ccc([N+](=O)[O-])cc1</smiles>

\section{1-(Diazo(phenyl)methyl)-4-nitrobenzene (8b)}

Prepared using general procedure, ((4-nitrophenyl)(phenyl)methylene)hydrazone (270 mg, 1.10 mmol) was used to afford the titled diazo compound (194 mg, $74 \%$ yield). ${ }^{1} \mathrm{H}$ NMR (600 MHz, $\left.\mathrm{CDCl}_{3}\right) \delta 7.78(\mathrm{~d}, J=8.99 \mathrm{~Hz}, 2 \mathrm{H}), 7.07-7.00(\mathrm{~m}, 2 \mathrm{H}), 6.95(\mathrm{t}, J=7.46 \mathrm{~Hz}, 1 \mathrm{H}), 6.88(\mathrm{dd}, J=$ $8.32,1.25 \mathrm{~Hz}, 2 \mathrm{H}), 6.52(\mathrm{~d}, J=8.97 \mathrm{~Hz}, 2 \mathrm{H})$. This is a known compound in literature. ${ }^{9}$<smiles>COc1ccc(C(=N)c2ccccc2)cc1</smiles>

\section{1-(Diazo(phenyl)methyl)-4-methoxybenzene (8c)}

Prepared using general procedure, 4-methoxyphenyl)(phenyl)methylene)hydrazone (250 mg, 1.10 mmol) was used to afford the titled diazo compound (198 mg, 80\% yield). ${ }^{1} \mathrm{H}$ NMR $(500 \mathrm{MHz}$, $\left.\mathrm{CDCl}_{3}\right) \delta 7.38-7.33(\mathrm{~m}, 2 \mathrm{H}), 7.28(\mathrm{~d}, J=8.97 \mathrm{~Hz}, 2 \mathrm{H}), 7.25-7.18(\mathrm{~m}, 2 \mathrm{H}), 7.17-7.11(\mathrm{~m}, 1 \mathrm{H})$, $6.98(\mathrm{~d}, J=9.01 \mathrm{~Hz}, 2 \mathrm{H}), 3.84(\mathrm{~s}, 3 \mathrm{H})$. This compound is known in the literature. ${ }^{9}$ 
<smiles>COc1ccc(C(=N)c2ccc([N+](=O)[O-])cc2)cc1</smiles>

1-(Diazo(4-methoxyphenyl)methyl)-4-nitrobenzene (8d)

Prepared using general procedure, ((4-methoxyphenyl)(4-nitrophenyl)methylene)hydrazone (300 mg, $1.1 \mathrm{mmol}$ ) was used to afford the titled diazo compound (231 mg, 78\% yield). ${ }^{1} \mathrm{H}$ $\operatorname{NMR}\left(600 \mathrm{MHz}, \mathrm{CDCl}_{3}\right) \delta 7.94(\mathrm{~d}, J=8.99 \mathrm{~Hz}, 2 \mathrm{H}), 6.96(\mathrm{~d}, J=8.76 \mathrm{~Hz}, 2 \mathrm{H}), 6.80(\mathrm{~d}, J=8.76$ $\mathrm{Hz}, 2 \mathrm{H}), 6.64(\mathrm{~d}, J=8.96 \mathrm{~Hz}, 2 \mathrm{H}), 3.37$ (s, 3H). ${ }^{13} \mathrm{C} \mathrm{NMR}\left(126 \mathrm{MHz}, \mathrm{CDCl}_{3}\right) \delta 159.55,143.90$, $139.77,129.56,124.59,122.20,118.47,115.21,55.45$, the signal due to $\mathrm{CN}_{2}$ was not observed. IR (in $\mathrm{CDCl}_{3}$ ) 2836, 2035, 1585, 1505, 1319, 1248, 1105, 850, 830, 748, 690. HRMS (+p NSI) calculated for $\mathrm{C}_{14} \mathrm{H}_{12} \mathrm{O}_{3} \mathrm{~N}\left[\mathrm{M}+\mathrm{H}-\mathrm{N}_{2}\right]^{+} 242.0822$ found 242.0810 . Melting point: 93-94 ${ }^{\circ} \mathrm{C}$.<smiles>N#Cc1ccccc1Cl</smiles>

$8 \mathrm{e}$

\section{1-Chloro-2-(diazo(phenyl)methyl)benzene (8e)}

Prepared using general procedure, ((2-chlorophenyl)(phenyl)methylene)hydrazone (250 mg, 1.1 $\mathrm{mmol}$ ) was used to afford the titled diazo compound (213 $\mathrm{mg}, 83 \%$ yield). This compound is unstable and was immediately subject to the cyclopropanation reaction and was not stable enough to obtain a pure ${ }^{13} \mathrm{C}$ NMR. ${ }^{1} \mathrm{H}$ NMR $\left(400 \mathrm{MHz}, \mathrm{CDCl}_{3}\right) \delta 7.52-7.46(\mathrm{~m}, 2 \mathrm{H}), 7.36-7.29(\mathrm{~m}$, $4 \mathrm{H}), 7.12-7.07(\mathrm{~m}, 1 \mathrm{H}), 7.03-6.99(\mathrm{~m}, 2 \mathrm{H})$. This is a known compound in literature. ${ }^{7}$<smiles>N#Cc1ccc([N+](=O)[O-])cc1Cl</smiles>

$8 f$

2-Chloro-1-(diazo(phenyl)methyl)-4-nitrobenzene (8f)

Prepared using general procedure, (2-chloro-4-nitrophenyl)(phenyl)methylene)hydrazone (303 mg, $1.10 \mathrm{mmol}$ ) was used to afford the titled diazo compound ( $250 \mathrm{mg}, 81 \%$ yield). ${ }^{1} \mathrm{H}$ NMR $\left(600 \mathrm{MHz}, \mathrm{CDCl}_{3}\right) \delta 8.35(\mathrm{~d}, J=2.4 \mathrm{~Hz}, 1 \mathrm{H}), 8.10(\mathrm{dd}, J=8.7,2.4 \mathrm{~Hz}, 1 \mathrm{H}), 7.53(\mathrm{~d}, J=8.8 \mathrm{~Hz}$, $1 \mathrm{H}), 7.40(\mathrm{dd}, J=8.4,7.5 \mathrm{~Hz}, 2 \mathrm{H}), 7.25-7.20(\mathrm{~m}, 1 \mathrm{H}), 7.16-7.13(\mathrm{~m}, 2 \mathrm{H}) .{ }^{13} \mathrm{C} \mathrm{NMR}(150 \mathrm{MHz}$, $\left.\mathrm{CDCl}_{3}\right) \delta 146.41,135.79,132.96,129.69,129.44,128.87,126.45,126.28,124.43,122.02$, the signal due to $\mathrm{CN}_{2}$ was not observed. IR (in $\mathrm{CDCl}_{3}$ ) 3099, 2053, 1674, 1520, 1344, 1280, 878, 765, 744, 696. HRMS (+p NSI) calculated for $\mathrm{C}_{13} \mathrm{H}_{9} \mathrm{ClNO}_{2}\left[\mathrm{M}+\mathrm{H}_{-} \mathrm{N}_{2}\right]^{+} 246.0327$ found 246.03148 . Melting point: $72-74{ }^{\circ} \mathrm{C}$.<smiles>COc1ccc(C(=N)c2ccccc2Cl)cc1</smiles>

$8 \mathrm{~g}$

1-Chloro-2-(diazo(4-methoxyphenyl)methyl)benzene (8g)

Prepared using general procedure, ((2-chlorophenyl)(4-methoxyphenyl)methylene)hydrazone (287 $\mathrm{mg}, 1.10 \mathrm{mmol})$ was used to afford the titled diazo compound (216 $\mathrm{mg}, 76 \%$ yield). The desired diazo was sensitive to acid and light, and was not stable enough to obtain a pure ${ }^{13} \mathrm{C} N M R$. 
Diazo compound was prepared, concentrated in vacuum in the dark and immediately subject to the cyclopropanation reaction. ${ }^{1} \mathrm{H}$ NMR $\left(500 \mathrm{MHz}, \mathrm{CDCl}_{3}\right) \delta 7.47(\mathrm{dd}, J=7.9,1.5 \mathrm{~Hz}, 1 \mathrm{H}), 7.41$ $(\mathrm{dd}, J=7.5,2.0 \mathrm{~Hz}, 1 \mathrm{H}), 7.31-7.23(\mathrm{~m}, 2 \mathrm{H}), 6.97-6.89(\mathrm{~m}, 4 \mathrm{H}), 3.80(\mathrm{~s}, 3 \mathrm{H})$. The product is sensitive to acid and was unstable in chloroform and other deuterated solvents. 


\subsection{Dirhodium catalyzed cyclopropanation reaction (11a-g)}<smiles>[R][14c]1cccc(C(=[W])c2ccccc2)c1</smiles><smiles>C=Cc1ccccc1</smiles>

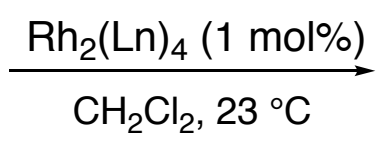<smiles>[R2]c1ccc(C2(c3ccccc3)CC2c2ccccc2)cc1</smiles>

4 or 10 equiv

\section{General procedure for cyclopropanation:}

To a flame-dried the desired dirhodium catalyst $\mathrm{Rh}_{2}(\mathrm{OAc})_{4}(0.88 \mathrm{mg}, 1 \mathrm{~mol} \%)$ added by stock ( $1.0 \mathrm{~mL}$ of $0.002 \mathrm{M}$ in $\mathrm{CH}_{2} \mathrm{Cl}_{2}$ or $\mathrm{Rh}_{2}(S-\mathrm{NTTL})_{4}(2.90 \mathrm{mg}, 1 \mathrm{~mol} \%)$ or $\mathrm{Rh}_{2}(S \text {-PTAD })_{4}(3.12 \mathrm{mg}$, $1 \mathrm{~mol} \%$ ) was added, and the reaction vial was purged with argon three times and dissolved in $\mathrm{CH}_{2} \mathrm{Cl}_{2}(1.0 \mathrm{~mL})$ and was charged with styrene. The corresponding diazo compound $(0.2 \mathrm{mmol})$ was weighed in a $20 \mathrm{~mL}$ vial and dissolved in dry degassed DCM $(4.0 \mathrm{~mL})$. The diazo compound solution was then added to the reaction vial dropwise over 1 hour at room temperature $\left(23^{\circ} \mathrm{C}\right)$ via a syringe pump. Reaction was stopped after $14 \mathrm{~h}$ and concentrated under vacuum for crude ${ }^{1} \mathrm{H}$ NMR to determine the diasteromeric ratio. The product was purified via flash column chromatography (a mixture of diastereomers and enantiomers) with a gradient of 0 to $25 \%$ diethyl ether in hexanes.

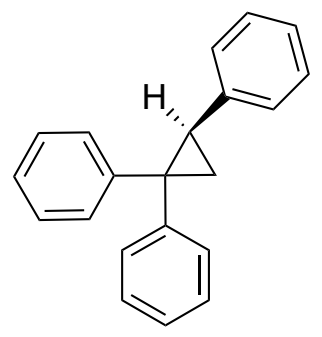

$11 \mathrm{a}$

(R)-cyclopropane-1,1,2-triyltribenzene (11a)

Prepared using general procedure, styrene $(0.23 \mathrm{~mL}, 2.0 \mathrm{mmol}, 10$ equiv) with diazo compound $8 \mathbf{a}(39 \mathrm{mg}, 0.50 \mathrm{mmol})$ using dirhodium catalyst $(1 \mathrm{~mol} \%)$ with a reaction time of $14 \mathrm{~h}$. Flash chromatography (gradient 0 to $10 \%$ diethyl ether/hexanes) to afford the titled product $11 \mathrm{a} .{ }^{1} \mathrm{H}$ NMR $\left(600 \mathrm{MHz} \mathrm{CDCl}_{3}\right) \delta 7.27(\mathrm{tdd}, J=10.0,7.3,1.8 \mathrm{~Hz}, 4 \mathrm{H}), 7.16(\mathrm{td}, J=7.0,1.9 \mathrm{~Hz}, 1 \mathrm{H})$, $7.12-7.02(\mathrm{~m}, 8 \mathrm{H}), 6.87-6.84(\mathrm{~m}, 2 \mathrm{H}), 2.85$ (ddd, $J=8.6,6.5,1.7 \mathrm{~Hz}, 1 \mathrm{H}), 1.99-1.96(\mathrm{~m}, 1 \mathrm{H})$, $1.80(\mathrm{ddd}, J=9.4,5.3,1.7 \mathrm{~Hz}, 1 \mathrm{H}) .{ }^{13} \mathrm{C} \mathrm{NMR}\left(150 \mathrm{MHz}, \mathrm{CDCl}_{3}\right) \delta 147.00,140.20,138.69,131.16$, $128.33,127.92,127.90,127.62,127.41,126.21,125.89,125.57,39.31,32.37,20.86$. This is a known compound in literature. ${ }^{10}$

With $\mathrm{Rh}_{2}(\mathrm{OAc})_{4}$ : obtained 11a, $12 \mathrm{mg}$ in $21 \%$ yield.

With $\mathrm{Rh}_{2}(S \text {-PTAD) })_{4}$ : obtained 11a, $48 \mathrm{mg}, 88 \%$ yield, $92 \%$ ee.

With $\mathrm{Rh}_{2}(S \text {-NTTL })_{4}$ : obtained 11a, $47 \mathrm{mg}, 86 \%$ yield, $93 \%$ ee.

HPLC: (4900 column, hexane, $1 \mathrm{~mL} \mathrm{~min}^{-1} 0.5 \mathrm{mg} \mathrm{mL}^{-1} 30 \mathrm{~min}$, UV $230 \mathrm{~nm}$ ) retention times of 15. $4 \mathrm{~min}$ (minor) and $18.6 \mathrm{~min}$ (major) $92 \%$ ee with $\mathrm{Rh}_{2}(S$-PTAD) 4 and of $16.60 \mathrm{~min}$ (minor) and 19.7 min (major) $93 \%$ ee with $\mathrm{Rh}_{2}(S-\mathrm{NTTL}) 4$. $[\alpha]^{20}{ }_{\mathrm{D}}+133.8^{\circ}\left(\mathrm{c}=0.58, \mathrm{CHCl}_{3}\right)$ for $92 \%$ ee.<smiles>O=[N+]([O-])c1ccc(C2[C@H](c3ccccc3)C[C@H]2c2ccccc2)cc1</smiles>

11b 
((1S,2R)-1-(4-nitrophenyl)cyclopropane-1,2-diyl)dibenzene (11b)

Prepared using general procedure, styrene $(0.09 \mathrm{~mL}, 0.80 \mathrm{mmol}, 4$ equiv) with diazo compound 8b (48 mg, $0.50 \mathrm{mmol})$ using dirhodium catalyst $(1 \mathrm{~mol} \%)$ with a reaction time of $14 \mathrm{~h}$. Flash chromatography (gradient 0 to $15 \%$ diethyl ether/hexanes) afforded the titled product $\mathbf{1 1 b} .{ }^{1} \mathrm{H}$ NMR $\left(600 \mathrm{MHz}, \mathrm{CDCl}_{3}\right) \delta 8.13-8.09(\mathrm{~m}, 2 \mathrm{H}), 7.36-7.32(\mathrm{~m}, 2 \mathrm{H}), 7.25-7.01(\mathrm{~m}, 8 \mathrm{H}), 6.86-$ $6.83(\mathrm{~m}, 2 \mathrm{H}), 2.89(\mathrm{dd}, J=9.1,6.8 \mathrm{~Hz}, 1 \mathrm{H}), 2.13(\mathrm{dd}, J=6.8,5.7 \mathrm{~Hz}, 1 \mathrm{H}), 1.90(\mathrm{dd}, J=9.1,5.7$ $\mathrm{Hz}, 1 \mathrm{H}) .{ }^{13} \mathrm{C} \mathrm{NMR}\left(150 \mathrm{MHz}, \mathrm{CDCl}_{3}\right) \delta 154.54,145.93,138.38,137.49,131.48,128.31,127.90$, $127.81,127.55,127.00,126.13,123.63,39.02,34.08,22.07$. This is a known compound in literature. ${ }^{11}$

With $\mathrm{Rh}_{2}(\mathrm{OAc})_{4}$ : obtained 11b, $27 \mathrm{mg}, 43 \%$ yield.

With $\mathrm{Rh}_{2}(S \text {-PTAD })_{4}$ : obtained $\mathbf{1 1 b}, 57 \mathrm{mg}, 90 \%$ yield, $2: 1$ d.r. $92 \%$ ee.

With $\mathrm{Rh}_{2}(S \text {-NTTL })_{4}$ : obtained $11 \mathrm{~b}, 52 \mathrm{mg} 82 \%$ yield, $1.2: 1$ d.r. $87 \%$ ee.

With $\mathrm{Rh}_{2}(S \text {-DOSP })_{4}$ : obtained $11 \mathrm{~b}, 39 \mathrm{mg}, 62 \%$ yield, $12: 1$ d.r. $18 \%$ ee.

HPLC (ADH column, hexane, $1.0 \mathrm{~mL} \mathrm{~min}^{-1} 0.5 \mathrm{mg} \mathrm{mL}^{-1} 30 \mathrm{~min}$, UV $230 \mathrm{~nm}$ ) retention times of 12.65 min (major) and 14.12 min (minor) $92 \%$ ee with $\mathrm{Rh}_{2}(S \text {-PTAD) })_{4}, 12.70$ min (major) and 14.17 min (minor) $97 \%$ ee with $\mathrm{Rh}_{2}(S$-NTTL) 4 , and of 14.20 min (major) and 12.69 min (minor) $20 \%$ ee with $\mathrm{Rh}_{2}(S \text {-DOSP })_{4}$. The diastereomer was also visible with retention times of $14.12 \mathrm{~min}$ (major) and $15.61 \mathrm{~min}$ (minor) $68 \%$ ee with $\mathrm{Rh}_{2}(S$-PTAD) 4 and $14.2 \mathrm{~min}$ (major) and $15.64 \mathrm{~min}$ (minor) $87 \%$ ee with $\mathrm{Rh}_{2}(S$-NTTL) 4 , and of $12.69 \mathrm{~min}$ (major) and $13.56 \mathrm{~min}$ (minor) $20 \%$ ee with $\mathrm{Rh}_{2}(S \text {-DOSP })_{4}$.

$[\alpha]^{20} \mathrm{D}+154.1^{\circ}\left(\mathrm{c}=1.00, \mathrm{CHCl}_{3}\right)$ for $92 \%$ ee.

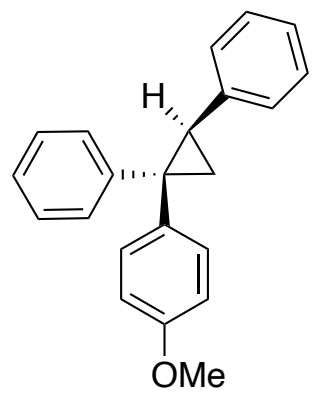

$11 \mathrm{C}$

((1R,2R)-1-(4-methoxyphenyl)cyclopropane-1,2-diyl)dibenzene (11c)

Prepared using general procedure, styrene $(0.09 \mathrm{~mL}, 0.80 \mathrm{mmol}, 4$ equiv) with diazo compound $8 c$ (45 mg, $0.50 \mathrm{mmol})$ using dirhodium catalyst $(1 \mathrm{~mol} \%)$ with a reaction time of $14 \mathrm{~h}$. Flash chromatography (gradient 0 to $15 \%$ diethyl ether/hexanes) afforded the titled product $11 \mathrm{c} .{ }^{1} \mathrm{H}$ NMR (400 MHz,, $\left.\mathrm{CDCl}_{3}\right) \delta 7.35-7.22(\mathrm{~m}, 4 \mathrm{H}), 7.14(\mathrm{~d}, J=14.77 \mathrm{~Hz}, 1 \mathrm{H}), 7.14-7.03(\mathrm{~m}, 3 \mathrm{H})$, $7.01(\mathrm{~d}, J=8.73 \mathrm{~Hz}, 2 \mathrm{H}), 6.92-6.80(\mathrm{~m}, 2 \mathrm{H}), 6.66(\mathrm{~d}, J=8.73 \mathrm{~Hz}, 2 \mathrm{H}), 3.71(\mathrm{~s}, 3 \mathrm{H}), 2.81(\mathrm{dd}, J$ $=9.00,6.58 \mathrm{~Hz}, 1 \mathrm{H}), 1.93(\mathrm{dd}, J=6.61,5.31 \mathrm{~Hz}, 1 \mathrm{H}), 1.80(\mathrm{dd}, J=8.99,5.31 \mathrm{~Hz}, 1 \mathrm{H})$. This is a known compound in literature. ${ }^{12}$

With $\mathrm{Rh}_{2}(\mathrm{OAc})_{4}$ : obtained 11c, $23 \mathrm{mg}, 39 \%$ yield)

With $\mathrm{Rh}_{2}(S \text {-PTAD) })_{4}$ : obtained 11c, $40 \mathrm{mg}, 66 \%$ yield, $20: 1$ d.r., $97 \%$ ee.

With $\mathrm{Rh}_{2}(S \text {-NTTL })_{4}$ : obtained 11c, $36 \mathrm{mg} 60 \%$ yield, $8: 1$ d.r., $98 \%$ ee.

With $\mathrm{Rh}_{2}(S \text {-DOSP })_{4}$ : obtained 11c, $21 \mathrm{mg}, 36 \%$ yield, $10: 1 \mathrm{dr}, 21 \%$ ee.

HPLC (ADH column, hexane, $1.5 \mathrm{~mL} \mathrm{~min}^{-1} 0.5 \mathrm{mg} \mathrm{mL}^{-1} 30 \mathrm{~min}$, UV $230 \mathrm{~nm}$ ) retention times of 4.6 min (minor) and 5.3 min (major) $97 \%$ ee with $\mathrm{Rh}_{2}(S \text {-PTAD) })_{4}, 4.6 \mathrm{~min}$ (minor) and $5.3 \mathrm{~min}$ (major) $98 \%$ ee with $\mathrm{Rh}_{2}\left(S\right.$-NTTL) 4 and of 4.6 min (minor) 5.3 min (major) $21 \%$ ee with $\mathrm{Rh}_{2}(S$ DOSP $)_{4}$.

$[\alpha]^{20} \mathrm{D}+99.0^{\circ}\left(\mathrm{c}=0.25, \mathrm{CHCl}_{3}\right)$ for $97 \%$ ee. 
<smiles>COc1ccc([C@H]2C[C@H]2c2ccccc2)cc1</smiles>

11d

1-methoxy-4-((1R,2R)-1-(4-nitrophenyl)-2-phenylcyclopropyl)benzene (11d)

Prepared using general procedure, styrene $(0.09 \mathrm{~mL}, 0.90 \mathrm{mmol}, 4$ equiv) with diazo compound $\mathbf{8 d}$ $(54 \mathrm{mg}, 0.50 \mathrm{mmol})$ using dirhodium catalyst $(1 \mathrm{~mol} \%)$ with a reaction time of $14 \mathrm{~h}$. Flash chromatography (gradient 0 to $15 \%$ diethyl ether/hexanes) afforded the titled product $11 d .{ }^{1} \mathrm{H}$ NMR $\left(600 \mathrm{MHz}, \mathrm{CDCl}_{3}\right) \delta 8.10(\mathrm{~d}, J=8.90 \mathrm{~Hz}, 2 \mathrm{H}), 7.30(\mathrm{~d}, J=8.96 \mathrm{~Hz}, 2 \mathrm{H}), 7.14-7.07(\mathrm{~m}$, $3 \mathrm{H}), 6.96(\mathrm{~d}, J=8.80 \mathrm{~Hz}, 2 \mathrm{H}), 6.89-6.80(\mathrm{~m}, 2 \mathrm{H}), 6.70(\mathrm{~d}, J=8.77 \mathrm{~Hz}, 2 \mathrm{H}), 3.73(\mathrm{~s}, 3 \mathrm{H}), 2.85$ $(\mathrm{dd}, J=9.1,6.8 \mathrm{~Hz}, 1 \mathrm{H}), 2.08(\mathrm{dd}, J=6.9,5.6 \mathrm{~Hz}, 1 \mathrm{H}), 1.89(\mathrm{dd}, J=9.1,5.7 \mathrm{~Hz}, 1 \mathrm{H}) .{ }^{13} \mathrm{C} \mathrm{NMR}$ $\left(150 \mathrm{MHz}, \mathrm{CDCl}_{3}\right) \delta 158.44,155.01,145.83,137.64,132.56,130.37,127.92,127.82,127.31$, $126.08, \quad 123.60,113.73,55.15,38.28,34.37,22.50$. IR(in $\left.\mathrm{CDCl}_{3}\right) \quad 3004,2932,2835$, 2360,1593,1510,1343,1245,1031,860, 757,697. HRMS $\left(+\mathrm{p}\right.$ APCl) calculated for $\mathrm{C}_{22} \mathrm{H}_{20} \mathrm{O}_{3} \mathrm{~N}$ $[\mathrm{M}+\mathrm{H}]^{+} 346.1438$ found 346.1431 .

With $\mathrm{Rh}_{2}(\mathrm{OAc})_{4}$ : obtained 11d, $50 \mathrm{mg}, 73 \%$ yield.

With $\mathrm{Rh}_{2}(S \text {-PTAD })_{4}$ : obtained 11d, $60 \mathrm{mg}, 88 \%$ yield, $>50: 1$ d.r., $92 \%$ ee.

With $\mathrm{Rh}_{2}(S \text {-NTTL })_{4}$ : obtained 11d, $62 \mathrm{mg} 90 \%$ yield, $>50: 1$ d.r. , $87 \%$ ee.

HPLC (ADH column, hexane, $1.0 \mathrm{~mL} \mathrm{~min}^{-1} 0.5 \mathrm{mg} \mathrm{mL}^{-1} 60 \mathrm{~min}$, UV $320 \mathrm{~nm}$ ) retention times of 26.9 min (minor) and 32.8 min (major) $92 \%$ ee with $\mathrm{Rh}_{2}(S \text {-PTAD) })_{4}$ and of $27.13 \mathrm{~min}$ (minor) and 33.74 min (major) $87 \%$ ee with $\mathrm{Rh}_{2}(S$-NTTL) 4 .

$[\alpha]^{20}{ }_{\mathrm{D}}+213.3^{\circ}\left(\mathrm{c}=1.00, \mathrm{CHCl}_{3}\right)$ for $92 \%$ ee.

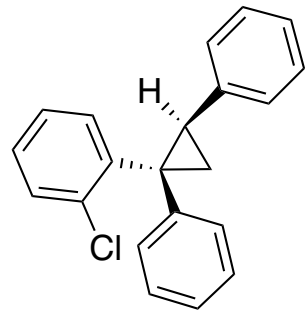

$11 \mathrm{e}$

((1S,2R)-1-(2-chlorophenyl)cyclopropane-1,2-diyl)dibenzene (11e)

Prepared using general procedure, styrene $(0.23 \mathrm{~mL}, 2.0 \mathrm{mmol}, 10$ equiv) with diazo compound $8 \mathrm{e}$ $(46 \mathrm{mg}, 0.50 \mathrm{mmol})$ using dirhodium catalyst $(1 \mathrm{~mol} \%)$ with a reaction time of $14 \mathrm{~h}$. Flash chromatography (gradient 0 to $5 \%$ diethyl ether/hexanes) afforded the titled product 11e. ${ }^{1} \mathrm{H} \mathrm{NMR}$ $\left(400 \mathrm{MHz}, \mathrm{CDCl}_{3}\right) \delta 7.74(\mathrm{dd}, J=7.6,1.7 \mathrm{~Hz}, 1 \mathrm{H}), 7.32(\mathrm{dd}, J=7.9,1.4 \mathrm{~Hz}, 2 \mathrm{H}), 7.22-6.96(\mathrm{~m}$, $11 \mathrm{H}), 2.89(\mathrm{dd}, J=9.2,6.6 \mathrm{~Hz}, 1 \mathrm{H}), 2.11(\mathrm{dd}, J=6.6,5.6 \mathrm{~Hz}, 1 \mathrm{H}), 1.72(\mathrm{dd}, J=9.2,5.6 \mathrm{~Hz}, 1 \mathrm{H})$. ${ }^{13} \mathrm{C}$ NMR $\left(100 \mathrm{MHz}, \mathrm{CDCl}_{3}\right) \delta 143.93,139.22,138.27,135.43,132.28,130.21,130.11,128.29$, $128.02,127.67,127.62,126.93,126.09,125.75,38.20,31.45,19.65$. This is a known compound in literature. ${ }^{10}$

With $\mathrm{Rh}_{2}(\mathrm{OAc})_{4}$ : obtained 11e, $24 \mathrm{mg}, 39 \%$ yield.

With $\mathrm{Rh}_{2}(S \text {-PTAD) })_{4}$ : obtained 11e, $55 \mathrm{mg}, 91 \%$ yield, $>50: 1$ d.r., $85 \%$ ee.

With $\mathrm{Rh}_{2}(S \text {-NTTL })_{4}$ : obtained 11e, $44 \mathrm{mg} \mathrm{72 \%} \mathrm{yield,} \mathrm{,}>50: 1$ d.r., $82 \%$ ee.

HPLC (ADH column, hexane, $1.0 \mathrm{~mL} \mathrm{~min}^{-1} 0.5 \mathrm{mg} \mathrm{mL}^{-1} 30 \mathrm{~min}$, UV $230 \mathrm{~nm}$ ) retention times of $4.72 \mathrm{~min}$ (minor) and 5.00 min (major) $85 \%$ ee with $\mathrm{Rh}_{2}(S$-PTAD) 4 and of $4.72 \mathrm{~min}$ (minor) and 5.02 min (major) $82 \%$ ee with $\mathrm{Rh}_{2}(S \text {-NTTL })_{4}$.

$[\alpha]^{20}{ }_{\mathrm{D}}+141.4^{\circ}\left(\mathrm{c}=0.90, \mathrm{CHCl}_{3}\right)$ for $85 \%$ ee. 
<smiles>O=[N+]([O-])c1ccc([C@H]2C[C@H]2c2ccccc2)c(Cl)c1</smiles>

\section{((1S,2R)-1-(2-chloro-4-nitrophenyl)cyclopropane-1,2-diyl)dibenzene (11f)}

Prepared using general procedure, styrene $(0.23 \mathrm{~mL}, 2.0 \mathrm{mmol}, 10$ equiv) with diazo compound $\mathbf{8 f}$ $(55 \mathrm{mg}, 0.50 \mathrm{mmol})$ using dirhodium catalyst $(1 \mathrm{~mol} \%)$ with a reaction time of $14 \mathrm{~h}$. Flash chromatography (gradient 0 to $15 \%$ diethyl ether/hexanes) afforded the titled product $11 \mathrm{f} .{ }^{1} \mathrm{H}$ NMR $\left(600 \mathrm{MHz}, \mathrm{CDCl}_{3}\right) \delta 8.22(\mathrm{~d}, J=2.3 \mathrm{~Hz}, 1 \mathrm{H}), 8.14(\mathrm{dd}, J=8.5,2.4 \mathrm{~Hz}, 1 \mathrm{H}), 7.91(\mathrm{~d}, J=$ $8.5 \mathrm{~Hz}, 1 \mathrm{H}), 7.18-7.16(\mathrm{~m}, 2 \mathrm{H}), 7.14-7.11(\mathrm{~m}, 2 \mathrm{H}), 7.10-7.03(\mathrm{~m}, 4 \mathrm{H}), 6.99-6.95(\mathrm{~m}, 2 \mathrm{H})$, $2.90(\mathrm{dd}, J=9.3,6.7 \mathrm{~Hz}, 1 \mathrm{H}), 2.20(\mathrm{dd}, J=6.7,5.9 \mathrm{~Hz}, 1 \mathrm{H}), 1.74(\mathrm{dd}, J=9.3,5.9 \mathrm{~Hz}, 1 \mathrm{H}) .{ }^{13} \mathrm{C}$ NMR $\left(150 \mathrm{MHz}, \mathrm{CDCl}_{3}\right) \delta 150.96,146.96,137.53,137.21,136.48,132.82,130.28,128.17$, $127.96,127.83,126.78,126.16,125.45,121.97,38.07,31.46,19.59$. IR(in $\left.\mathrm{CDCl}_{3}\right) 3027$, $1519,1347,1121,892,775,740,720,697 . \mathrm{HRMS}\left(+\mathrm{p}\right.$ APCI) calculated for $\mathrm{C}_{21} \mathrm{H}_{16} \mathrm{ClNO}_{2}[\mathrm{M}+\mathrm{H}]^{+}$ 350.0943 found 350.09369 .

With $\mathrm{Rh}_{2}(\mathrm{OAc})_{4}$ : obtained 11f, $32 \mathrm{mg}, 46 \%$ yield)

With $\mathrm{Rh}_{2}(S \text {-PTAD })_{4}$ : obtained 11f, $64 \mathrm{mg}, 91 \%$ yield, $>50: 1$ d.r., $20 \%$ ee.

With $\mathrm{Rh}_{2}(S \text {-NTTL })_{4}$ : obtained 11f, $50 \mathrm{mg} 72 \%$ yield, $>50: 1$ d.r., $57 \%$ ee.

HPLC (ODH column, hexane, $1.5 \mathrm{~mL} \mathrm{~min}^{-1} 0.5 \mathrm{mg} \mathrm{mL}^{-1} 60 \mathrm{~min}$, UV $280 \mathrm{~nm}$ ) retention times of 21.04 min (major) and 31.17 min (minor) $20 \%$ ee with $\mathrm{Rh}_{2}(S$-PTAD) 4 and of 20.81 min (major) and 31.10 min (minor) $57 \%$ ee with $\mathrm{Rh}_{2}(S-\mathrm{NTTL})_{4}$.

$[\alpha]^{20}{ }_{\mathrm{D}}+107.5^{\circ}\left(\mathrm{c}=0.52, \mathrm{CHCl}_{3}\right)$ for $57 \%$ ee.

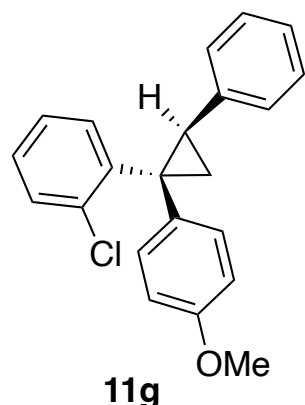

1-chloro-2-((1S,2R)-1-(4-methoxyphenyl)-2-phenylcyclopropyl)benzene (11g)

Prepared using general procedure, styrene $(0.23 \mathrm{~mL}, 2.0 \mathrm{mmol}, 4$ equiv) with diazo compound $\mathbf{8 g}$ $(52 \mathrm{mg}, 0.50 \mathrm{mmol})$ using dirhodium catalyst $(1 \mathrm{~mol} \%)$ with a reaction time of $14 \mathrm{~h}$. Flash chromatography (gradient 0 to $15 \%$ diethyl ether/hexanes) afforded the titled product $11 \mathrm{~g} .{ }^{1} \mathrm{H}$ $\operatorname{NMR}\left(600 \mathrm{MHz}, \mathrm{CDCl}_{3}\right) \delta 7.70(\mathrm{dd}, J=7.7,1.7 \mathrm{~Hz}, 1 \mathrm{H}), 7.31(\mathrm{dd}, J=7.9,1.3 \mathrm{~Hz}, 1 \mathrm{H}), 7.26(\mathrm{dd}$, $J=15.0,1.3 \mathrm{~Hz}, 1 \mathrm{H}), 7.16-7.10(\mathrm{~m}, 5 \mathrm{H}), 7.08-7.05(\mathrm{~m}, 1 \mathrm{H}), 7.00-6.97(\mathrm{~m}, 2 \mathrm{H}), 6.60(\mathrm{~d}, J=$ $8.85 \mathrm{~Hz}, 2 \mathrm{H}), 3.66(\mathrm{~s}, 3 \mathrm{H}), 2.83(\mathrm{dd}, J=9.1,6.6 \mathrm{~Hz}, 1 \mathrm{H}), 2.02(\mathrm{dd}, J=6.6,5.5 \mathrm{~Hz}, 1 \mathrm{H}), 1.71(\mathrm{dd}$, $\left.J=9.1,5.6 \mathrm{~Hz}, 1 \mathrm{H}) .{ }^{13} \mathrm{C} \mathrm{NMR} 150 \mathrm{MHz}, \mathrm{CDCl}_{3}\right) \delta 157.77,144.27,138.48,135.22,132.09$, $131.43,131.33,130.09,128.22,127.87,127.67,126.92,125.65,113.06,37.65,31.23,19.88$. IR (in $\mathrm{CDCl}_{3}$ ) 3003, 2834, 1608, 1510, 1470, 1244, 1176, 1032, 907, 734, 695, 614. HRMS (+p NSI) calculated for $\mathrm{C}_{22} \mathrm{H}_{19} \mathrm{ClONa}[\mathrm{M}+\mathrm{Na}]^{+} 357.1016$ found 357.1015 .

With $\mathrm{Rh}_{2}(\mathrm{OAc})_{4}$ : obtained 11g, $18 \mathrm{mg}, 27 \%$ yield

With $\mathrm{Rh}_{2}(S \text {-PTAD })_{4}$ : obtained $\mathbf{1 1 g}, 37 \mathrm{mg}, 56 \%$ yield, , $>50: 1$ d.r., $93 \%$ ee.

With $\mathrm{Rh}_{2}(S \text {-NTTL })_{4}$ : obtained $11 \mathrm{~g}, 23 \mathrm{mg} 34 \%$ yield, , $>50: 1$ d.r., $92 \%$ ee.

HPLC (ADH column, hexane, $1.0 \mathrm{~mL} \mathrm{~min}^{-1} 0.5 \mathrm{mg} \mathrm{mL}^{-1} 30 \mathrm{~min}$, UV $230 \mathrm{~nm}$ ) retention times6 min (minor) and 8.51 min (major) $93 \%$ ee with $\mathrm{Rh}_{2}(S$-PTAD) 4 and of $7.16 \mathrm{~min}$ (minor) and 8.50 min (major) $92 \%$ ee with $\mathrm{Rh}_{2}(S \text {-NTTL })_{4}$.

$[\alpha]^{20} \mathrm{D}+73.8^{\circ}\left(\mathrm{c}=0.58, \mathrm{CHCl}_{3}\right)$ for $93 \%$ ee. 


\section{Catalyst Screen}

To a flame-dried the desired dirhodium catalyst ( 1 mol \%) was added, and the reaction vial was purged with argon three times and dissolved in $\mathrm{CH}_{2} \mathrm{Cl}_{2}(1.0 \mathrm{~mL})$ and was charged with styrene (4 equiv.). The corresponding diazo compound $(0.20 \mathrm{mmol})$ was weighed in a $20 \mathrm{~mL}$ vial and dissolved in dry degassed DCM $(4.0 \mathrm{~mL})$. The diazo compound solution was then added to the reaction vial dropwise over 1 hour at room temperature $\left(23^{\circ} \mathrm{C}\right)$ via a syringe pump. The reaction was stopped after $14 \mathrm{~h}$ and concentrated under vacuum for crude ${ }^{1} \mathrm{H}$ NMR to determine the diasteromeric ratio. The product was purified via flash column chromatography (a mixture of diastereomers and enantiomers) with a gradient of 0 to $25 \%$ diethyl ether in hexanes.

\section{Table S1. Catalyst screen}

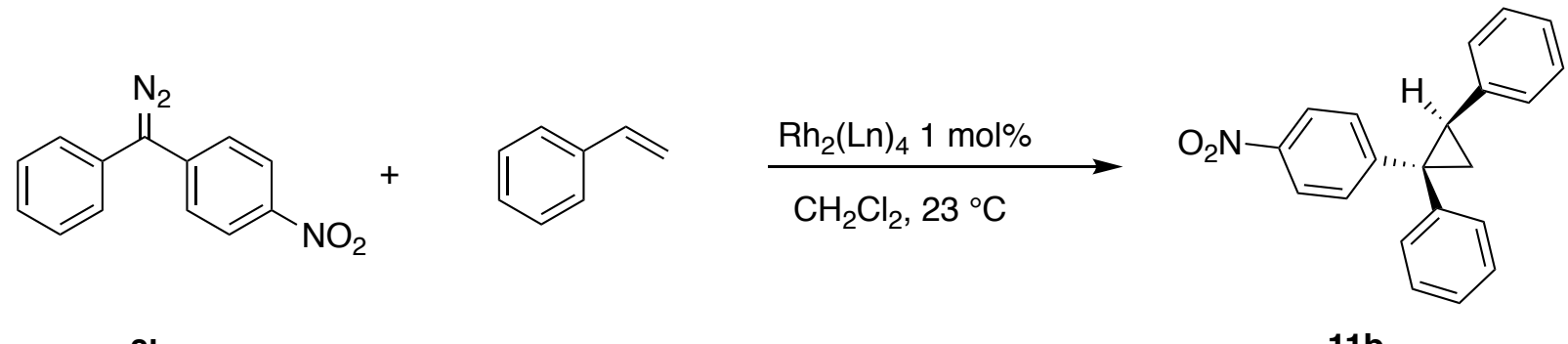

$8 b$

11b

\begin{tabular}{ccccc}
\hline Entry & Catalyst & yield $(\%)$ & d.r. & e.e. $(\%)$ major, minor \\
\hline 1 & $\mathrm{Rh}_{2}(\mathrm{OAC})_{4}$ & 43 & $6: 1$ & n.a. \\
2 & $\mathrm{Rh}_{2}(S-\mathrm{DOSP})_{4}$ & 62 & $12: 1$ & 19,18 \\
3 & $\mathrm{Rh}_{2}(\mathrm{~S}-\mathrm{PTAD})_{4}$ & 90 & $2: 1$ & 92,67 \\
4 & $\mathrm{Rh}_{2}(\mathrm{~S}-\mathrm{TCPTAD})_{4}$ & 31 & $1.5: 1$ & 81,71 \\
5 & $\mathrm{Rh}_{2}(\mathrm{~S}-\mathrm{TPPTTL})_{4}$ & 61 & $7: 1$ & 62,15 \\
6 & $\mathrm{Rh}_{2}(\mathrm{~S}-\mathrm{p}-\mathrm{PhTPCP})_{4}$ & 31 & $8: 1$ & 37,58 \\
7 & $\mathrm{Rh}_{2}(S-\mathrm{p}-\mathrm{BrTPCP})_{4}$ & 41 & $3: 1$ & 41,33 \\
8 & $\mathrm{Rh}_{2}(S-\mathrm{NTTL})_{4}$ & 82 & $1.2: 1$ & 97,89 \\
\hline
\end{tabular}




\section{Solvent Screen}

To a flame-dried $\mathrm{Rh}_{2}(S \text {-NTTL })_{4}(2.90 \mathrm{mg}, 1 \mathrm{~mol} \%)$ was added, and the reaction vial was purged with argon three times and dissolved in solvent $(1.0 \mathrm{~mL})$ and was charged with styrene (4 equiv.). The diazo compound $\mathbf{8 b}(48 \mathrm{mg}, 0.50 \mathrm{mmol}$ ) was weighed in a $20 \mathrm{~mL}$ vial and dissolved in dry degassed DCM $(4.0 \mathrm{~mL})$. The diazo compound solution was then added to the reaction vial dropwise over 1 hour at room temperature $\left(23^{\circ} \mathrm{C}\right)$ via a syringe pump. Reaction was stopped after $14 \mathrm{~h}$ and concentrated under vacuum for crude ${ }^{1} \mathrm{H}$ NMR to determine the diasteromeric ratio. The product was purified via flash column chromatography (a mixture of diastereomers and enantiomers) with a gradient of 0 to $25 \%$ diethyl ether in hexanes.

\section{Table S2. Solvent screen}<smiles></smiles>

$8 b$<smiles>C=Cc1ccccc1</smiles>
$\mathrm{Rh}_{2}(\mathrm{~S}-\mathrm{NTTL})_{4} 1 \mathrm{~mol} \%$ $\mathrm{CH}_{2} \mathrm{Cl}_{2}, 23^{\circ} \mathrm{C}$

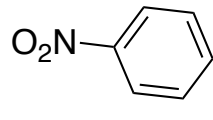

$\mathrm{H}$<smiles>C1CCCCC1</smiles><smiles>C/C=C\CCc1ccccc1</smiles>

11b

\begin{tabular}{ccccc}
\hline Entry & Solvent & yield $(\%)$ & d.r. & e.e. $(\%)$ major, minor \\
\hline 1 & Dichloromethane & 82 & $1.2: 1$ & 97,89 \\
2 & Toluene & 93 & $3: 1$ & 94,60 \\
3 & Trifluorotoluene & 87 & $2: 1$ & 96,33 \\
\hline
\end{tabular}




\section{NMR Spectra}

\subsection{NMR of purified compounds}
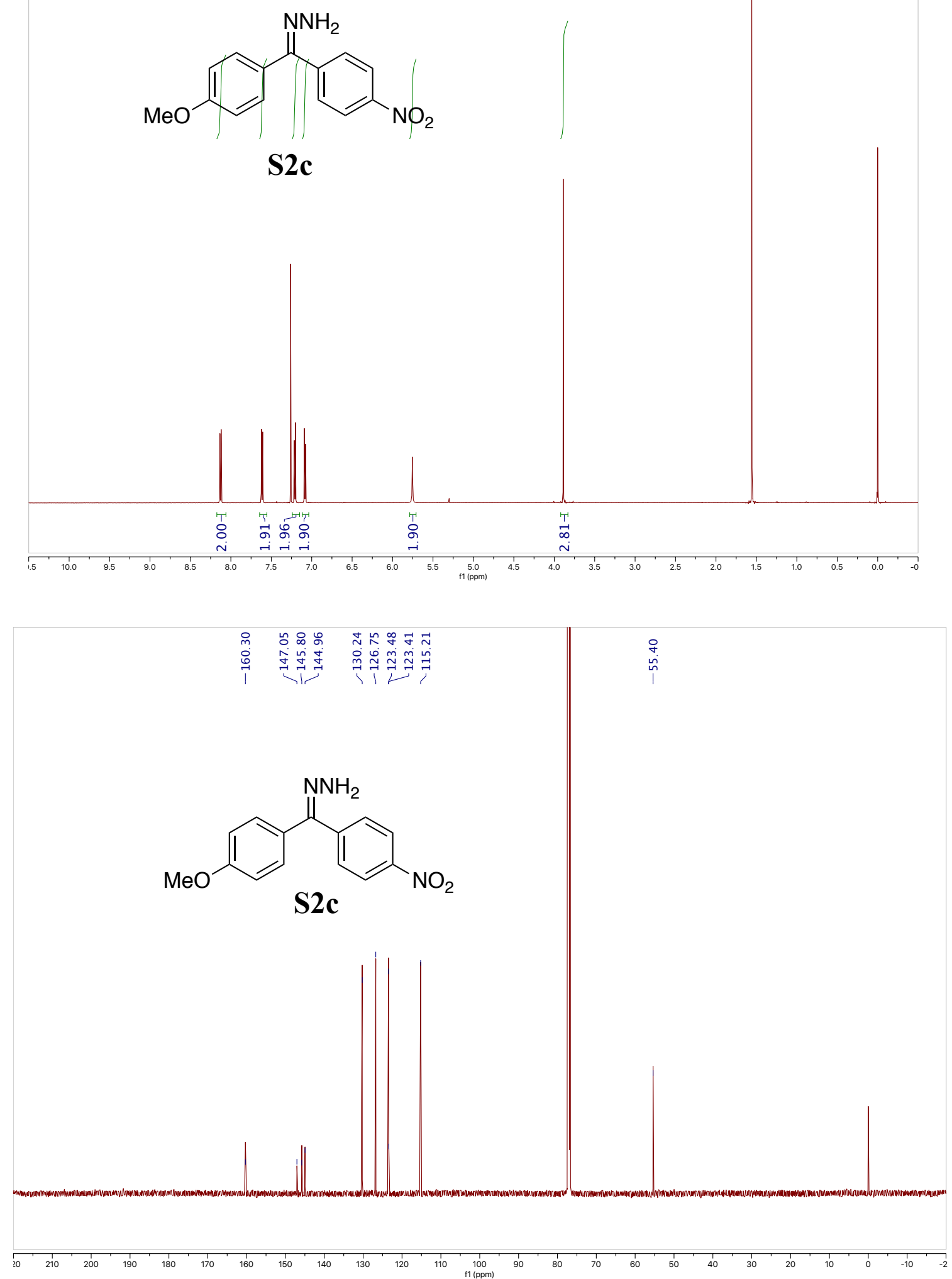

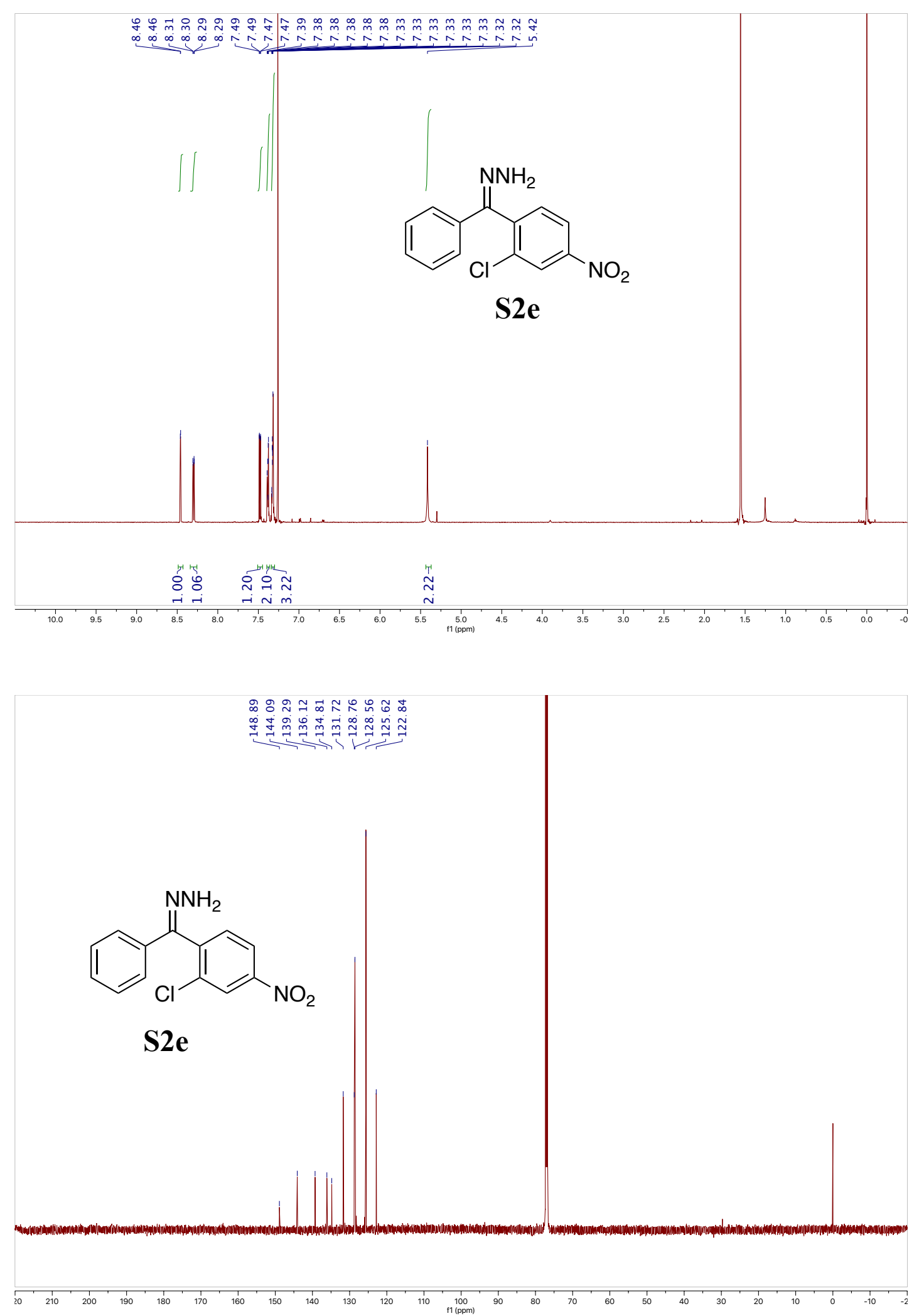

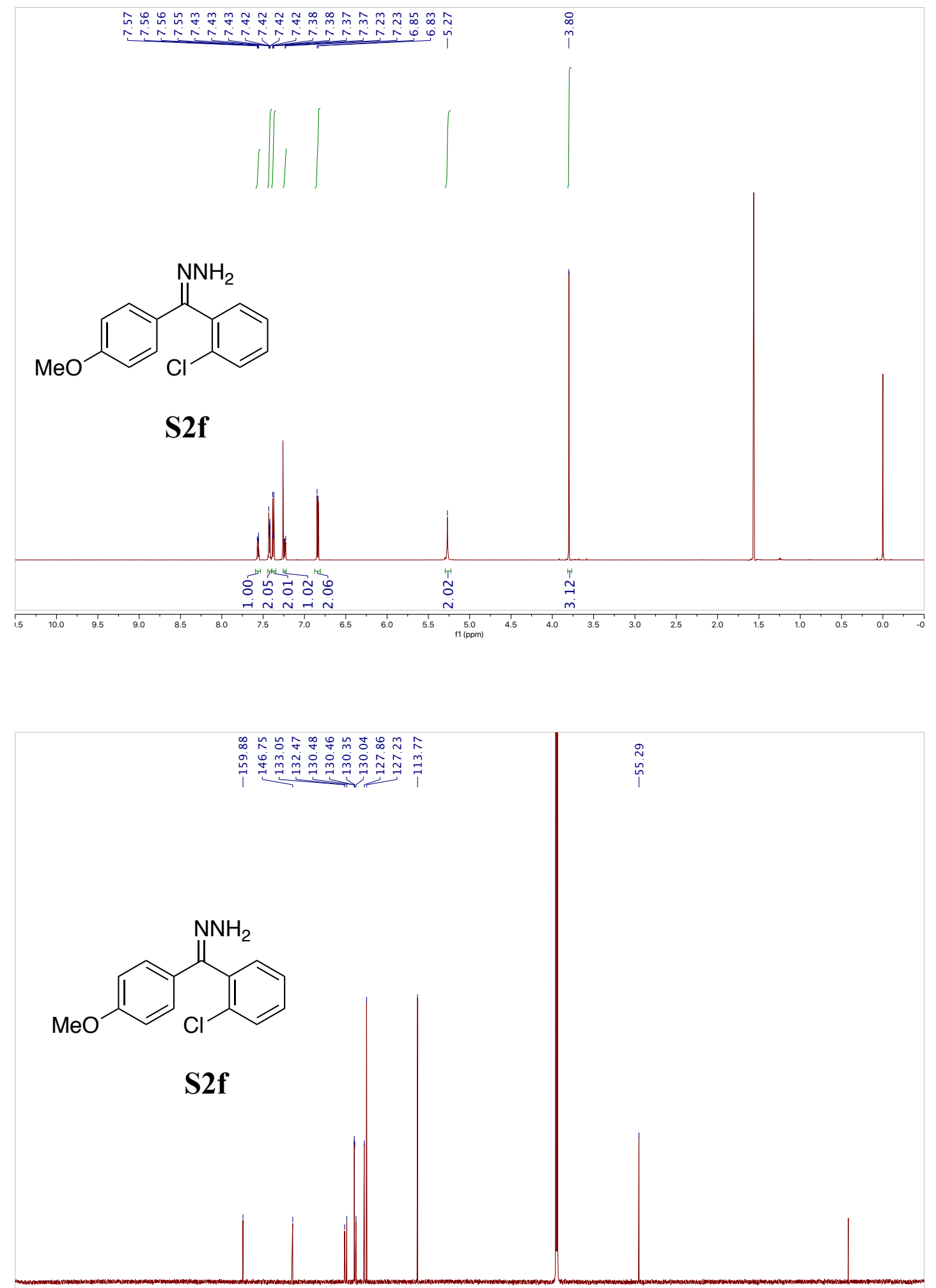

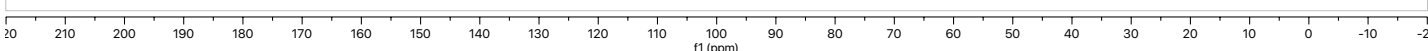



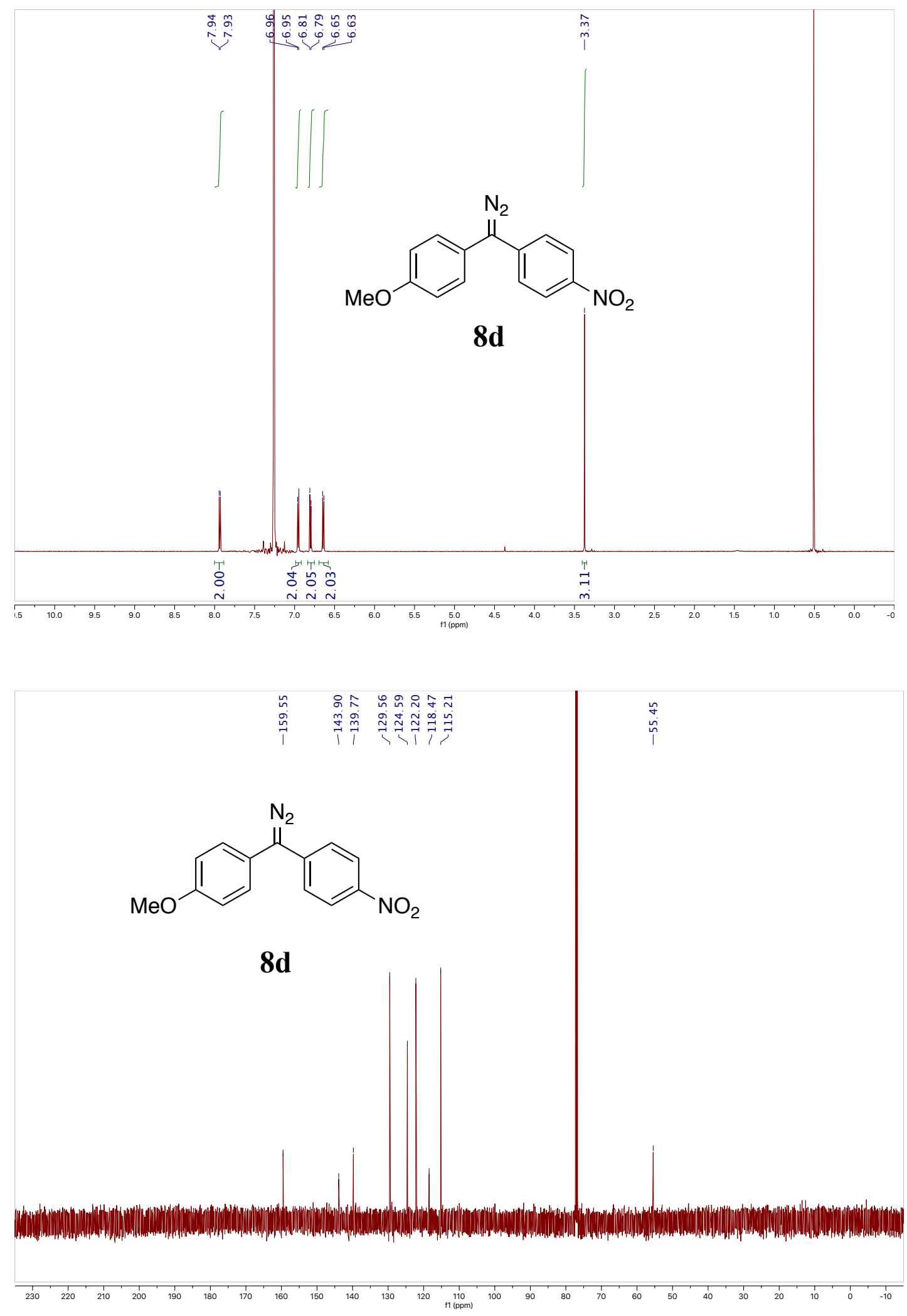

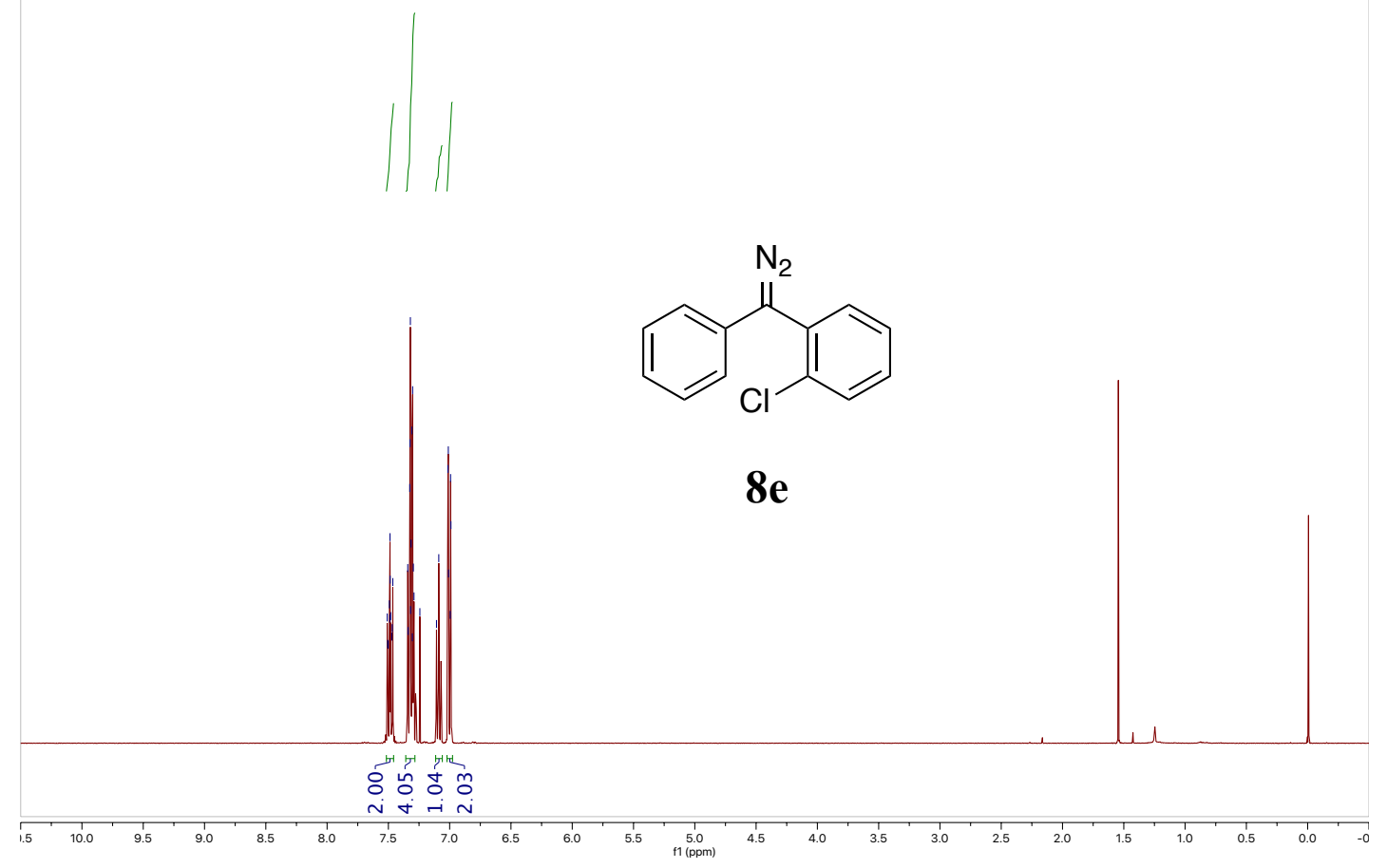

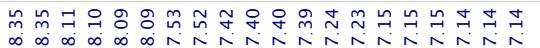

$\underbrace{\infty} \infty \infty^{\infty} \infty N^{\infty} N_{1}^{\infty}$
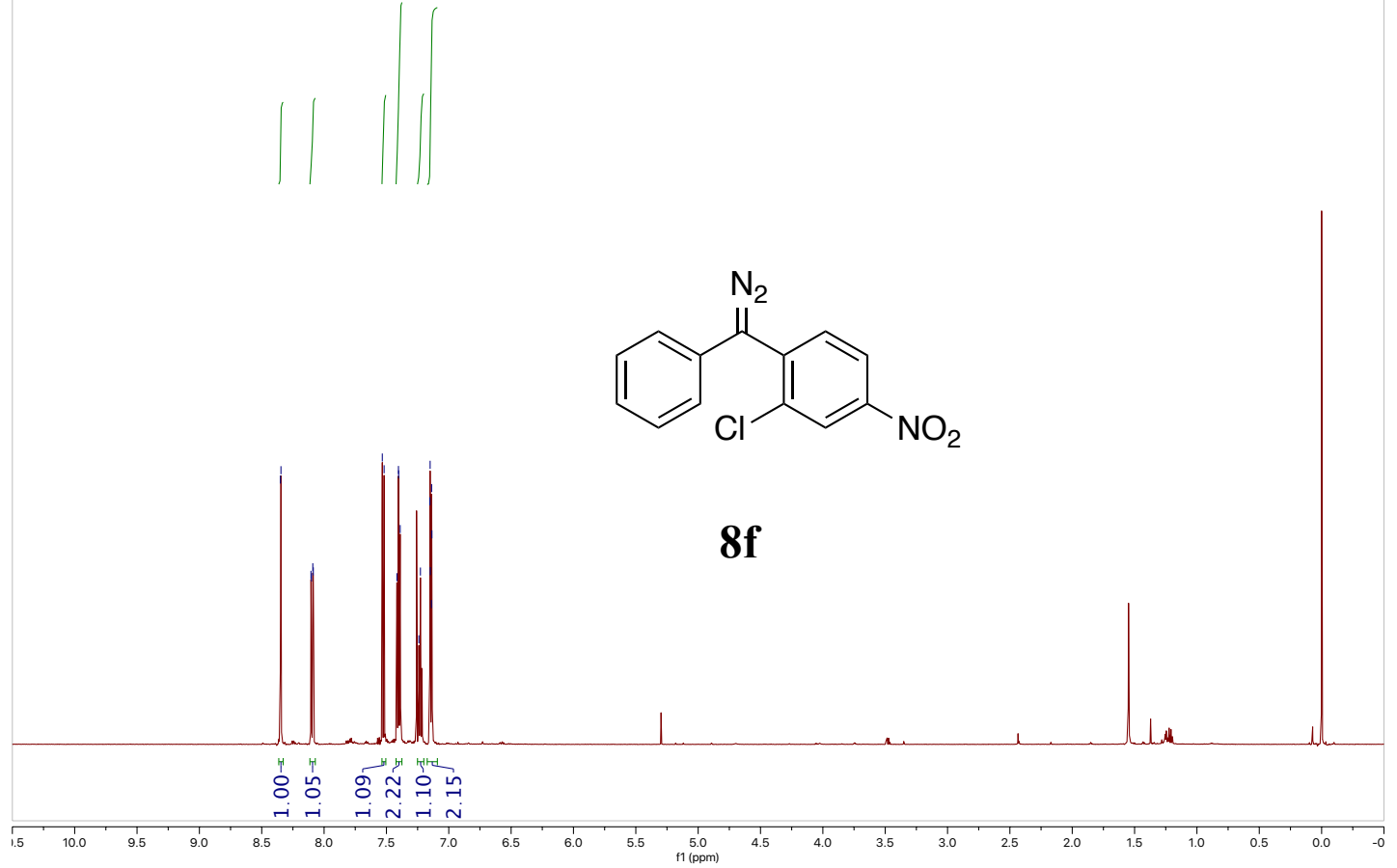


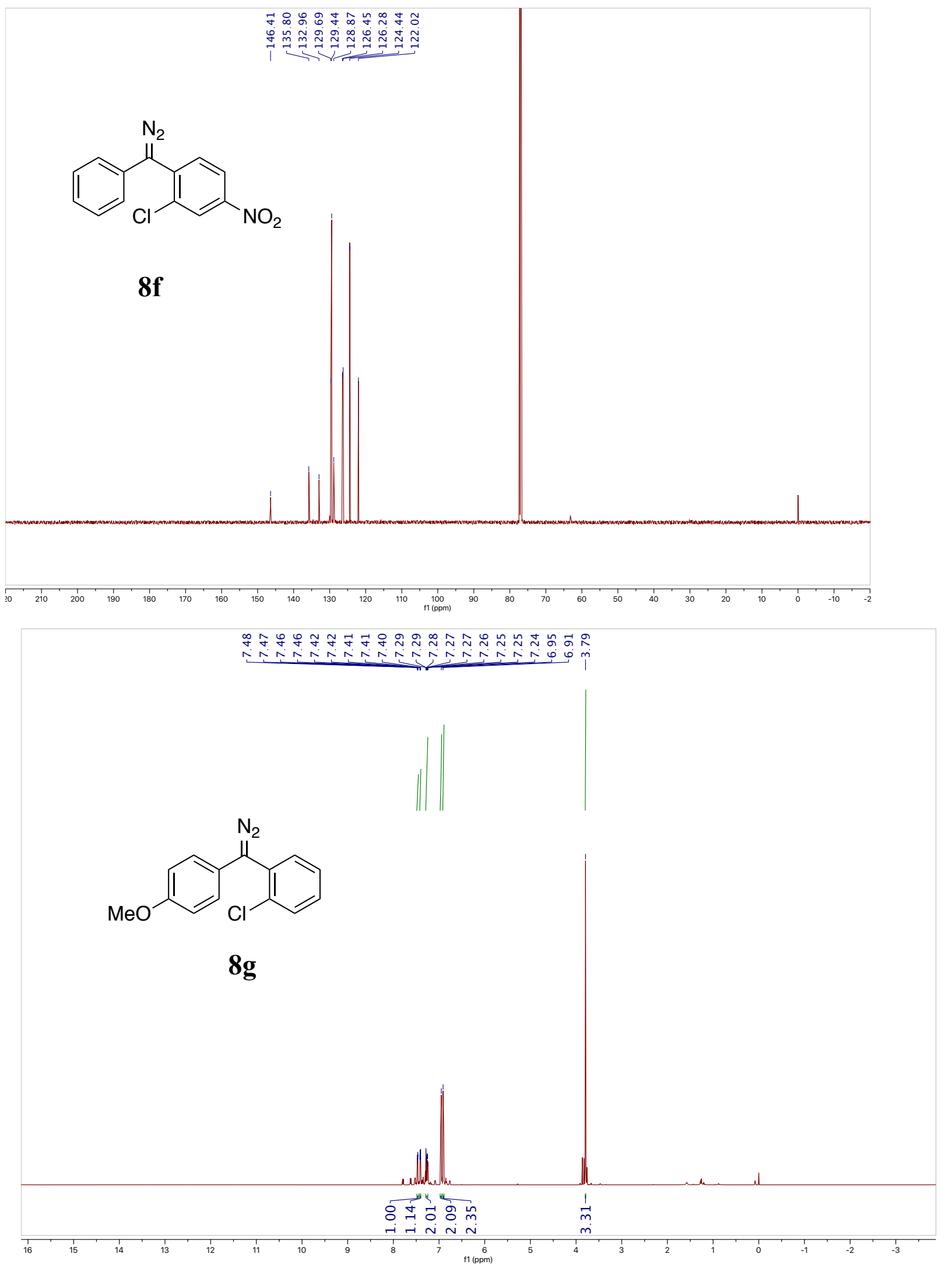



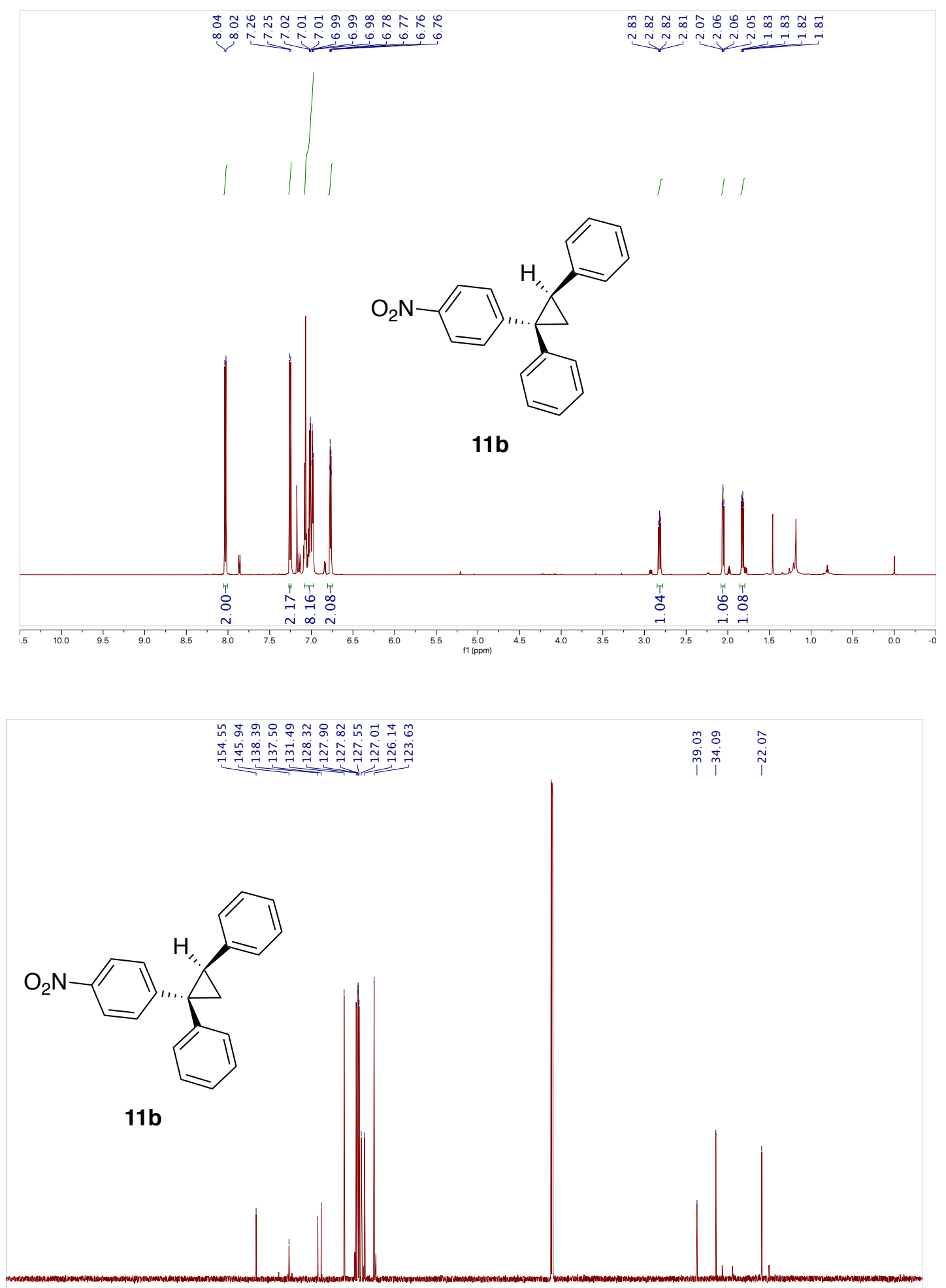


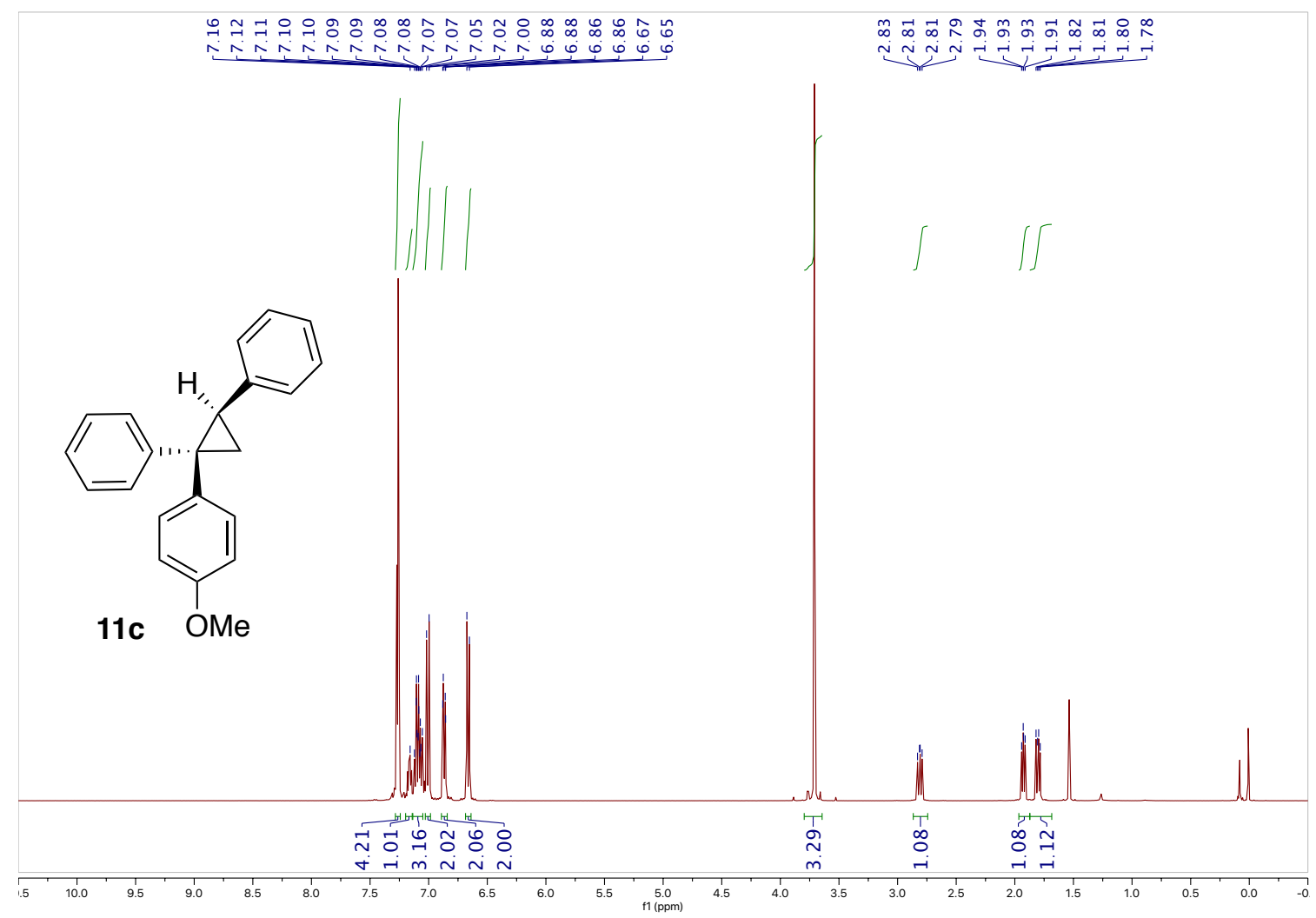

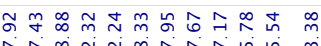

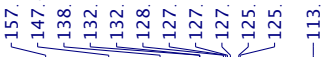

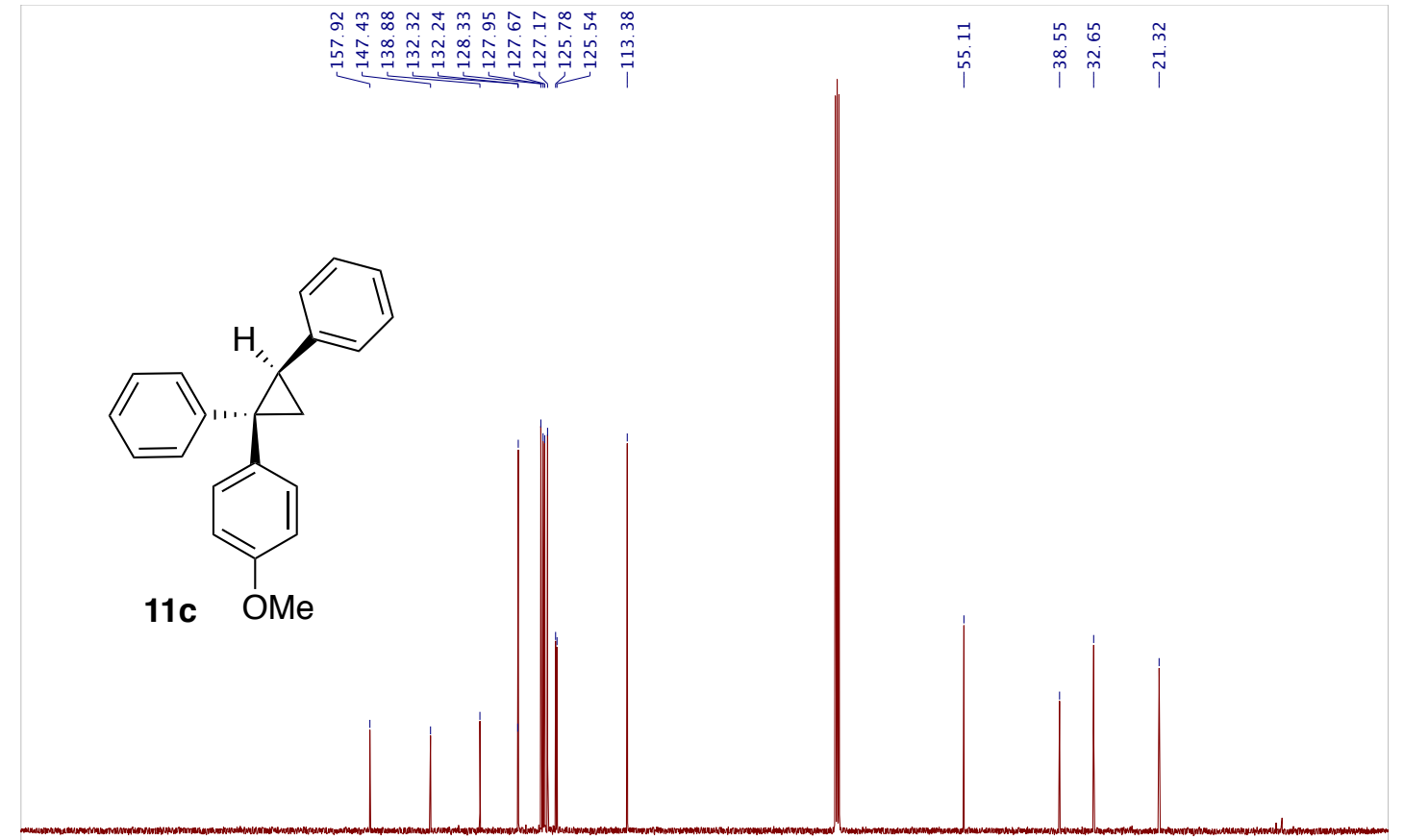



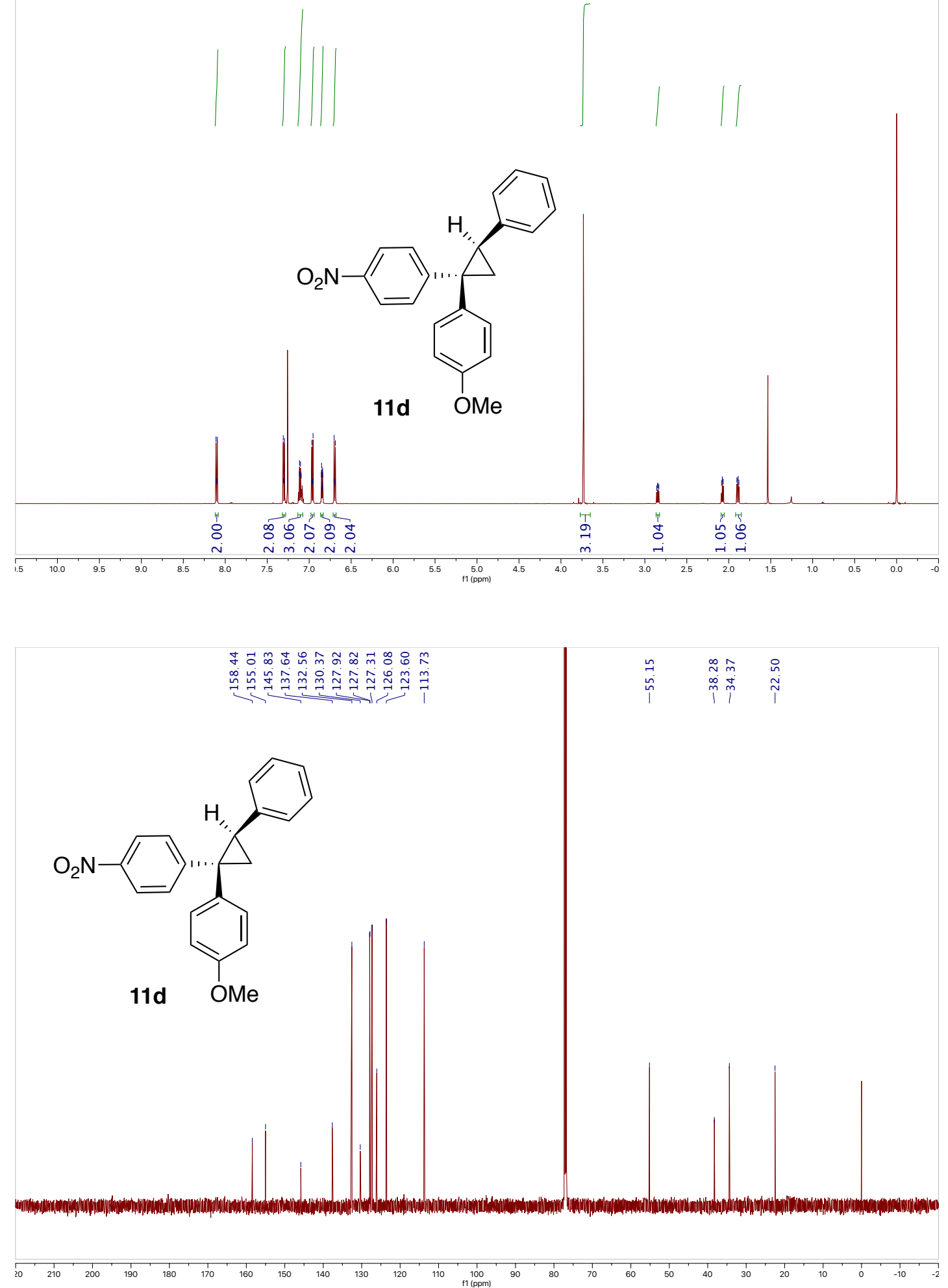

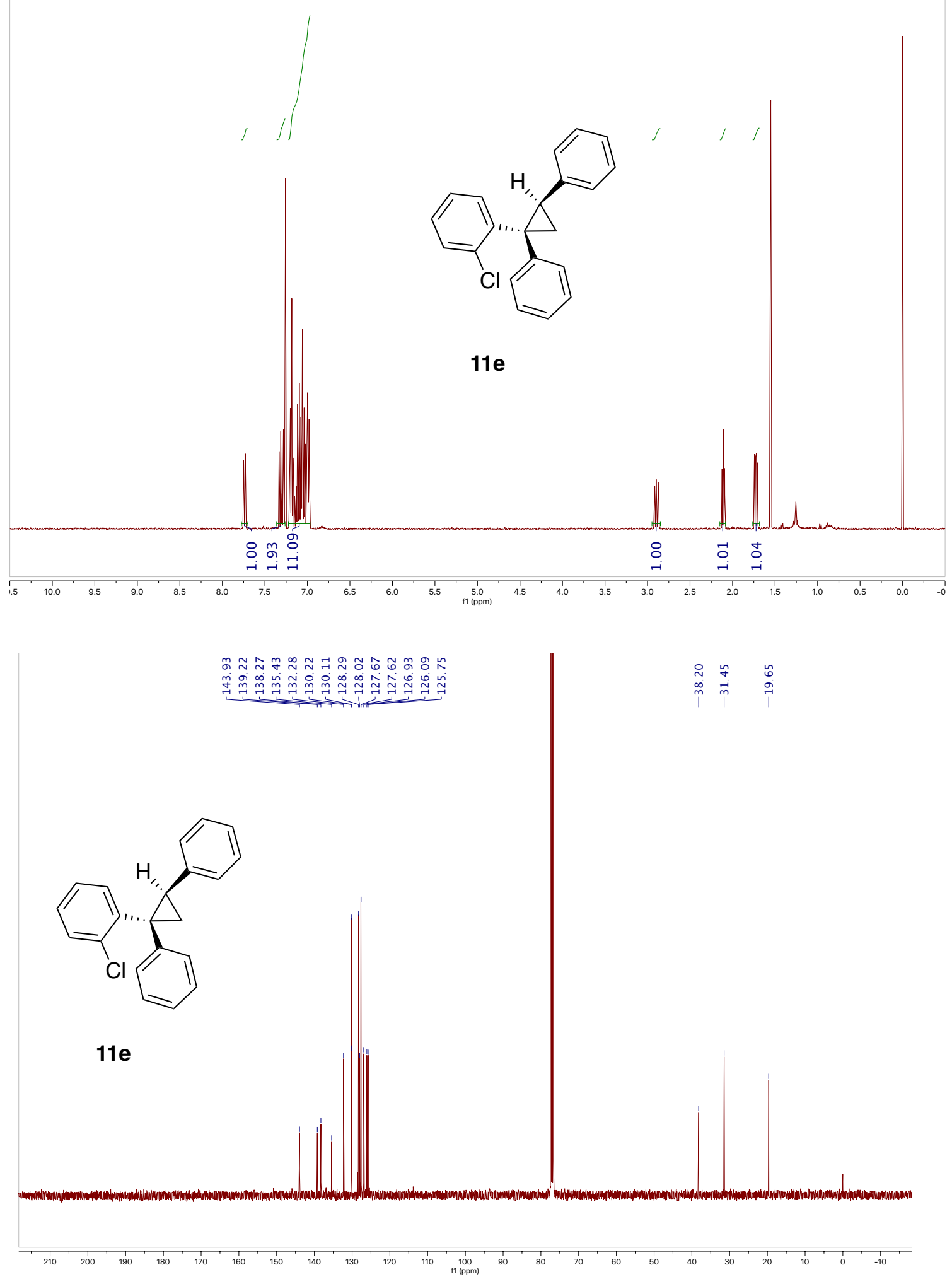

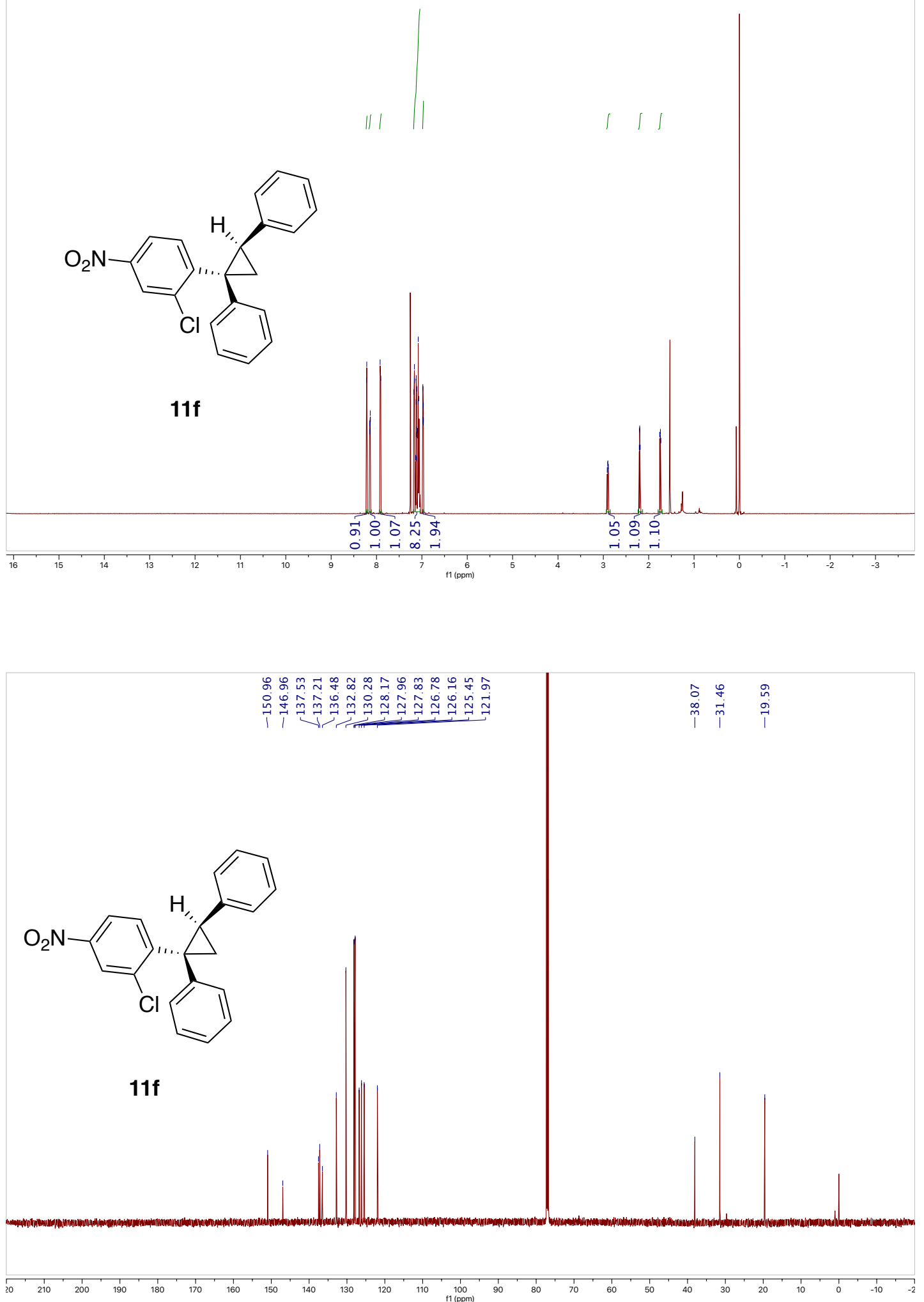

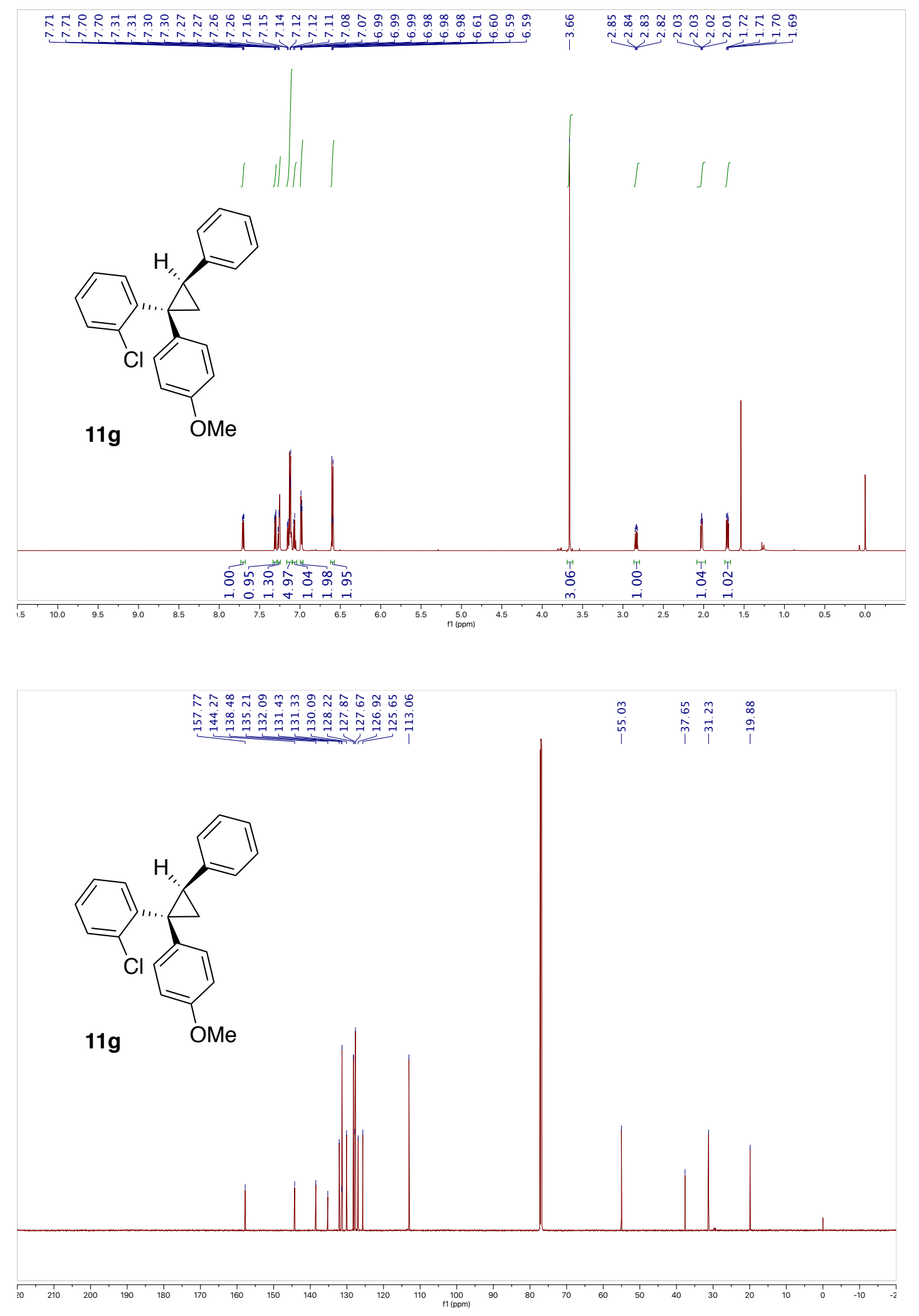
5.2 NMR for d.r. determinations<smiles>N#CC(c1ccccc1)c1ccc([N+](=O)[O-])cc1</smiles>

$8 b$<smiles>C=Cc1ccccc1</smiles>

$\mathrm{CH}_{2} \mathrm{Cl}_{2}, 23^{\circ} \mathrm{C}$ 


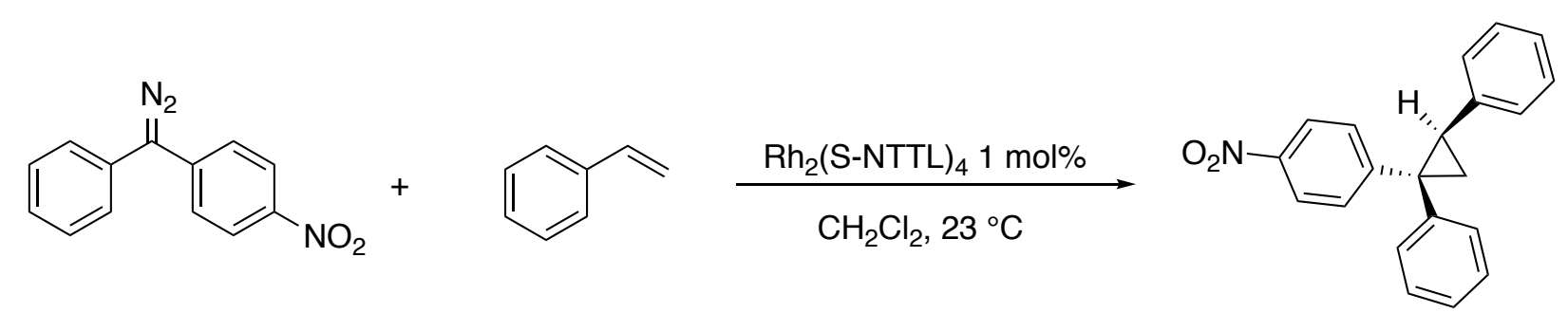

$8 b$

$11 b$

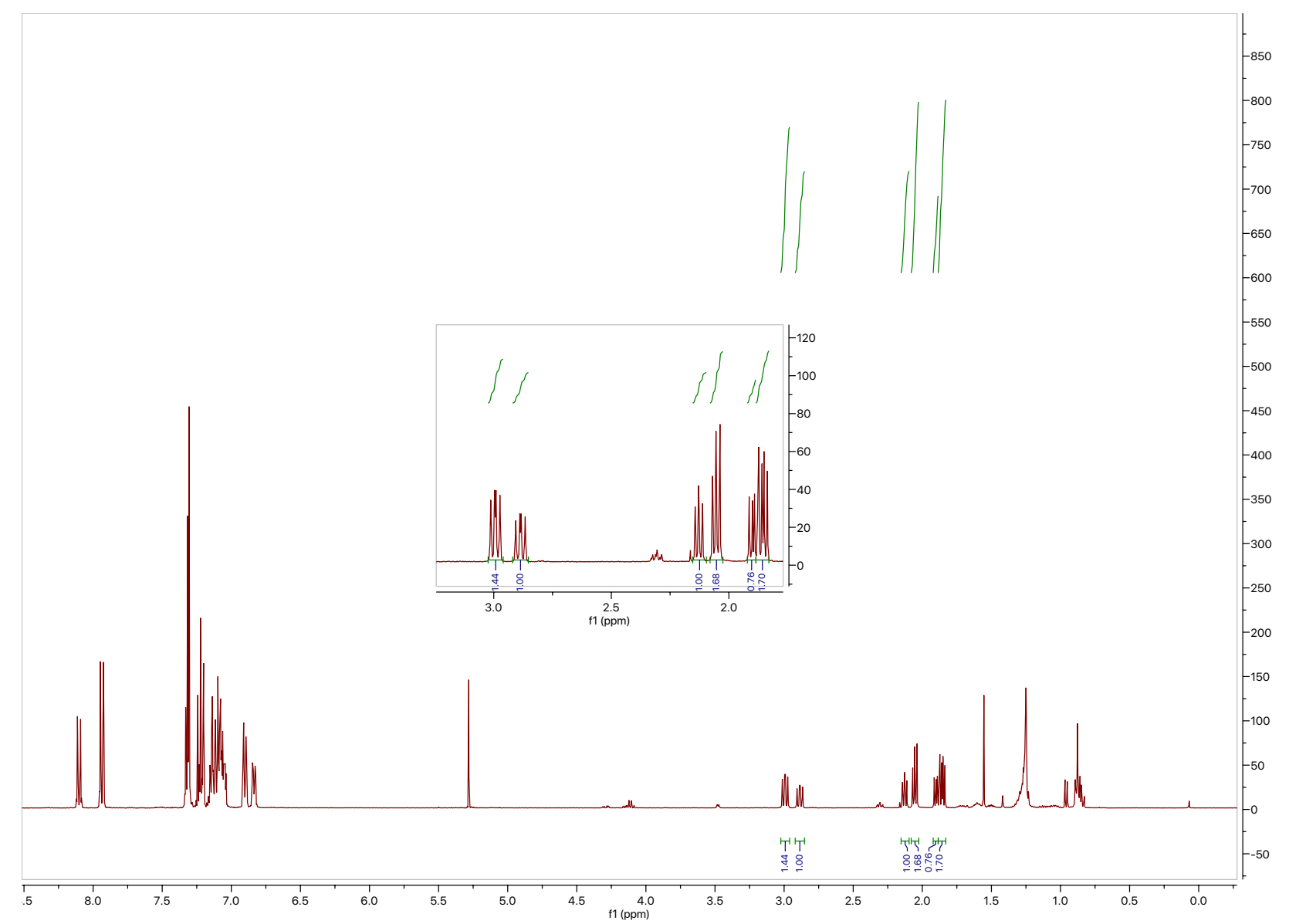




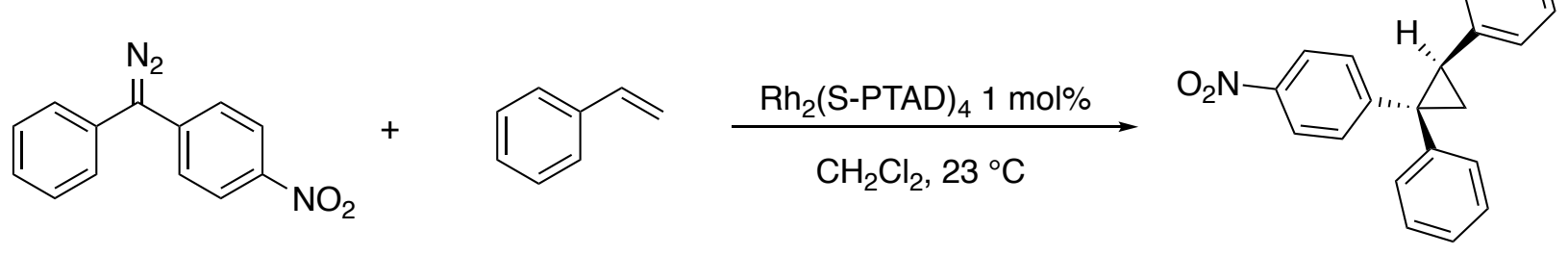

11b

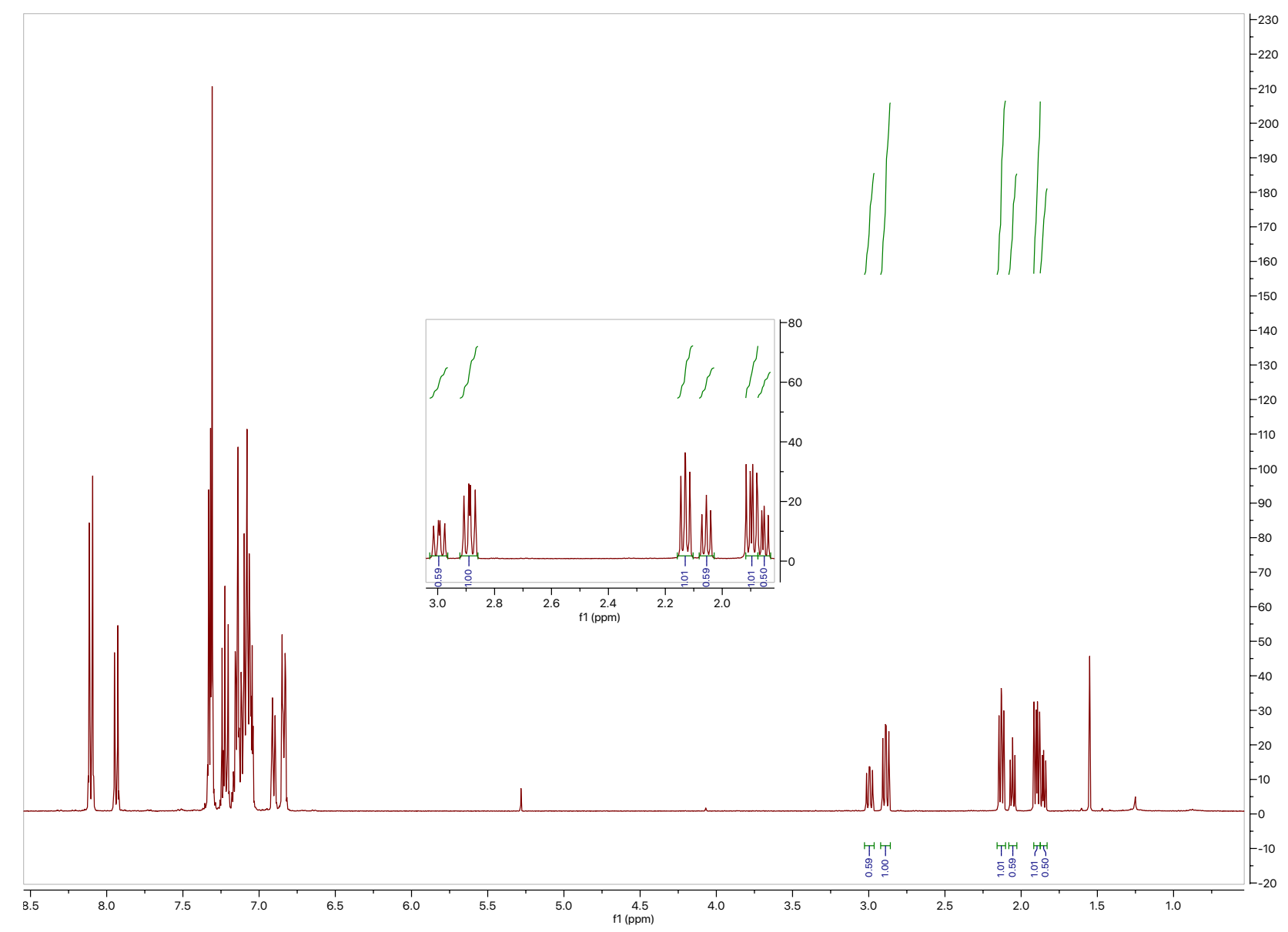



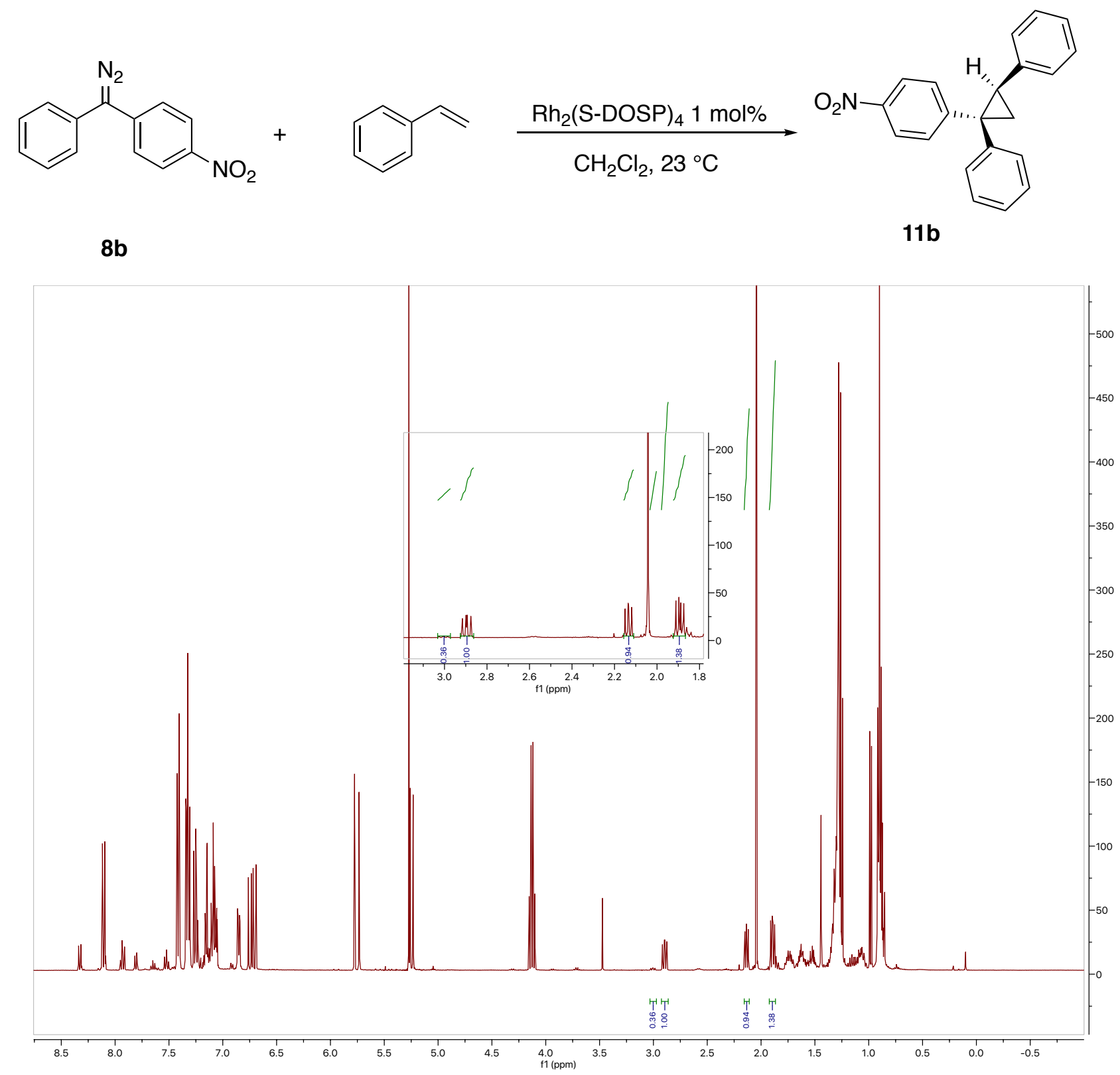
Stacked Spectrum

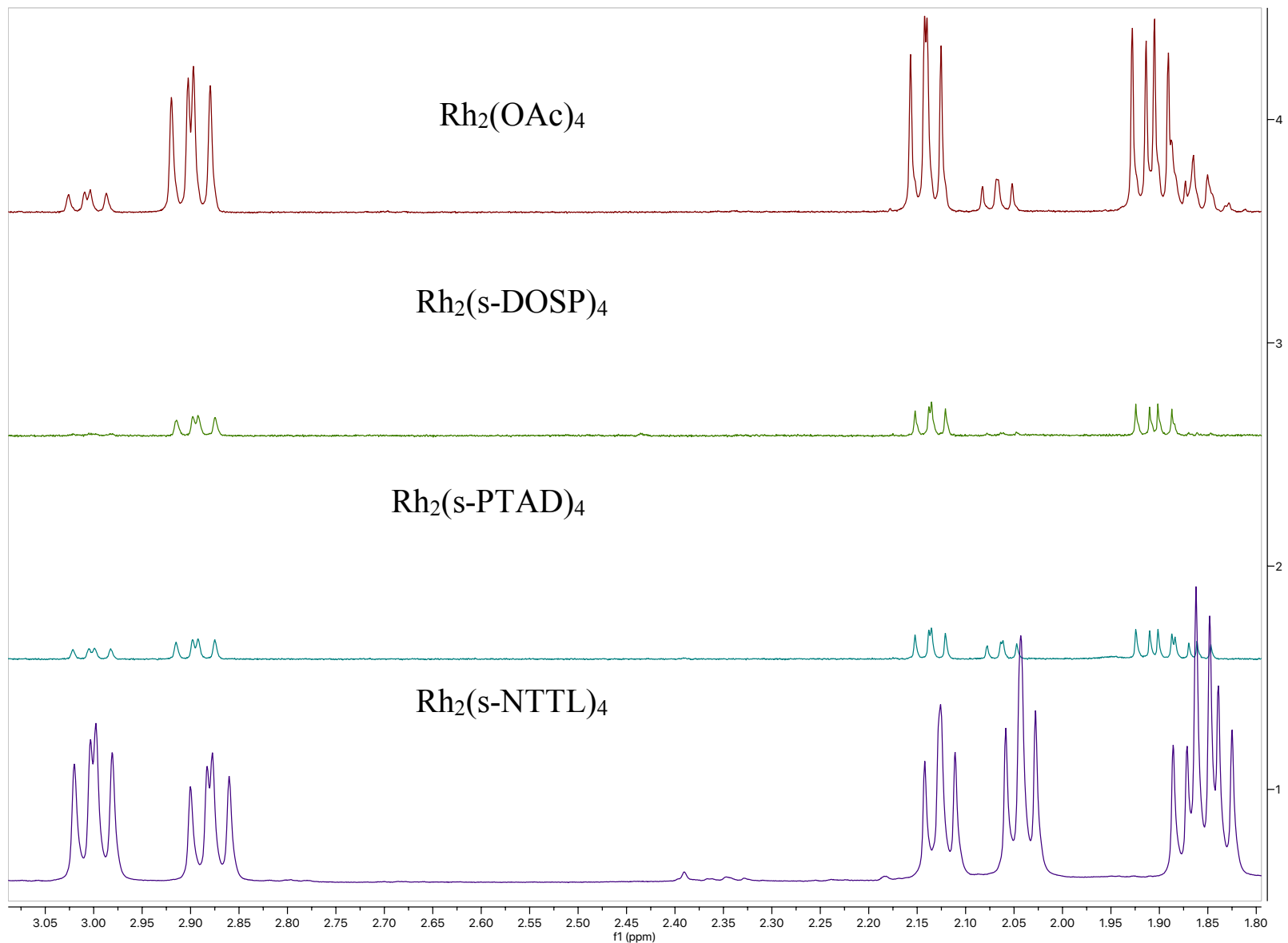


(c)

$8 c$

11c

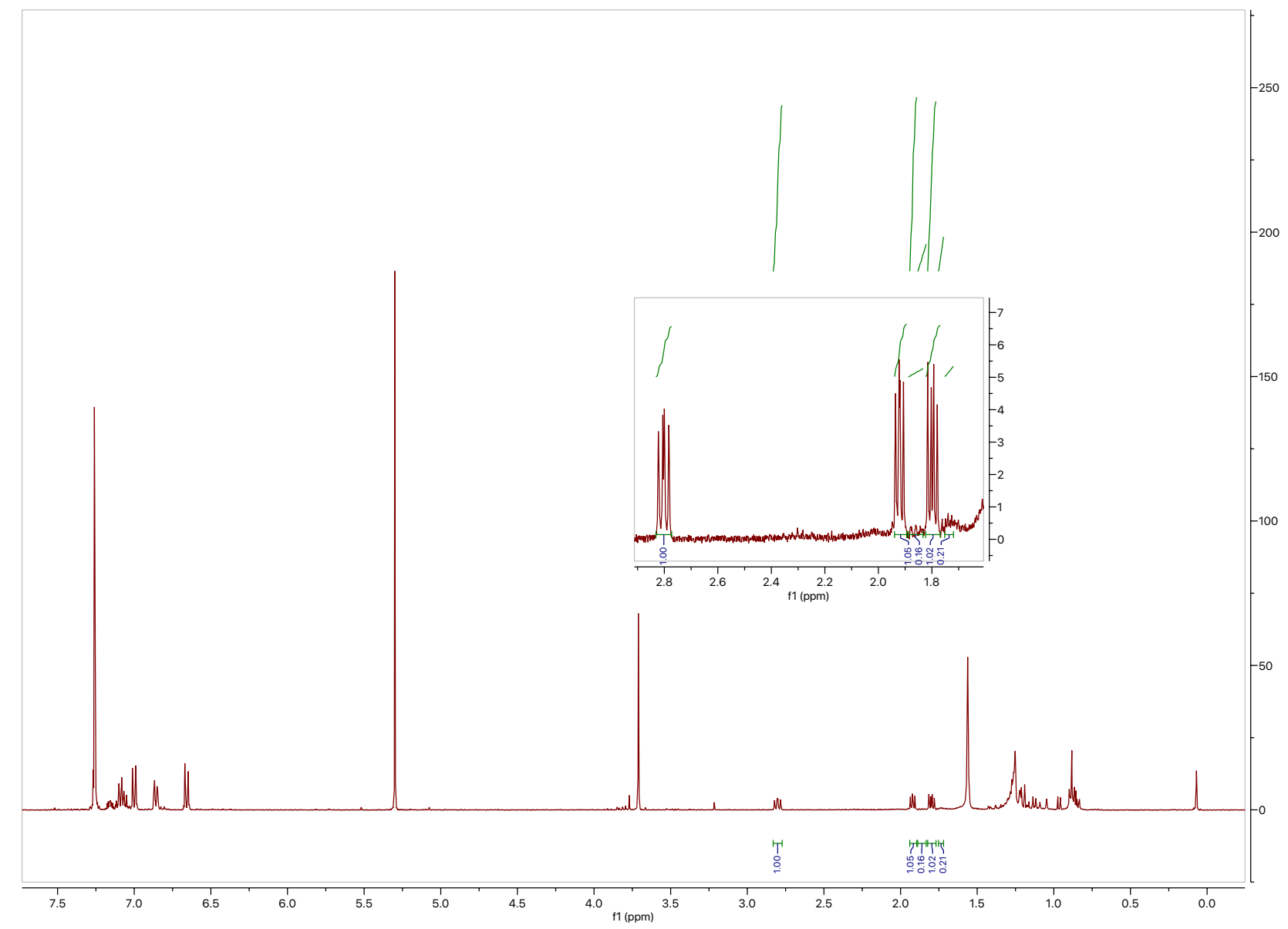




8c

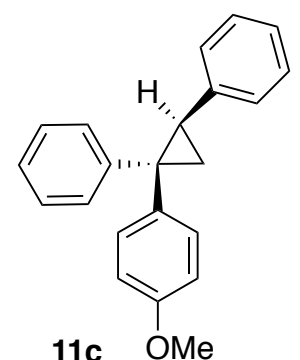

11c $\mathrm{OMe}$

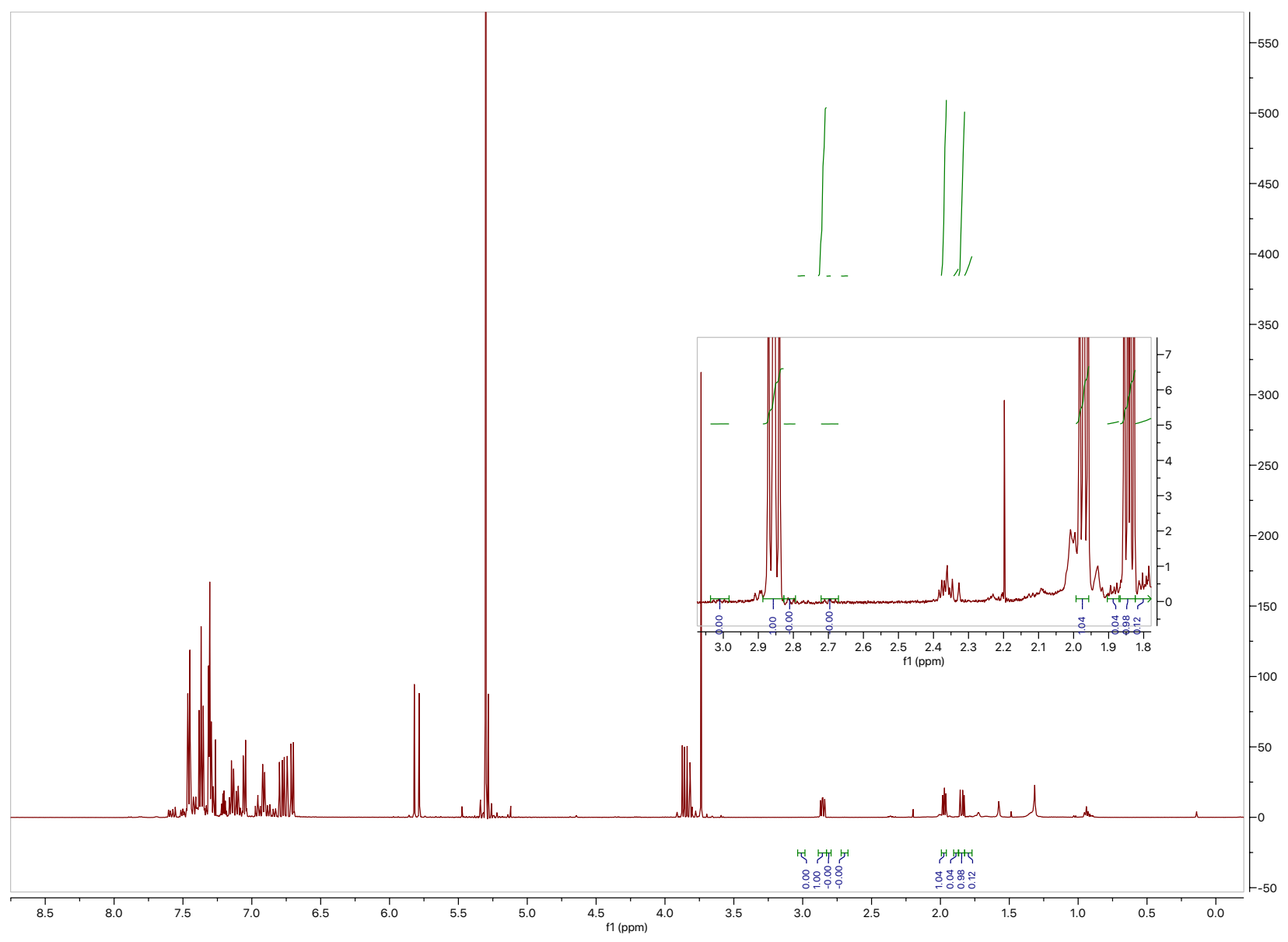




$8 c$

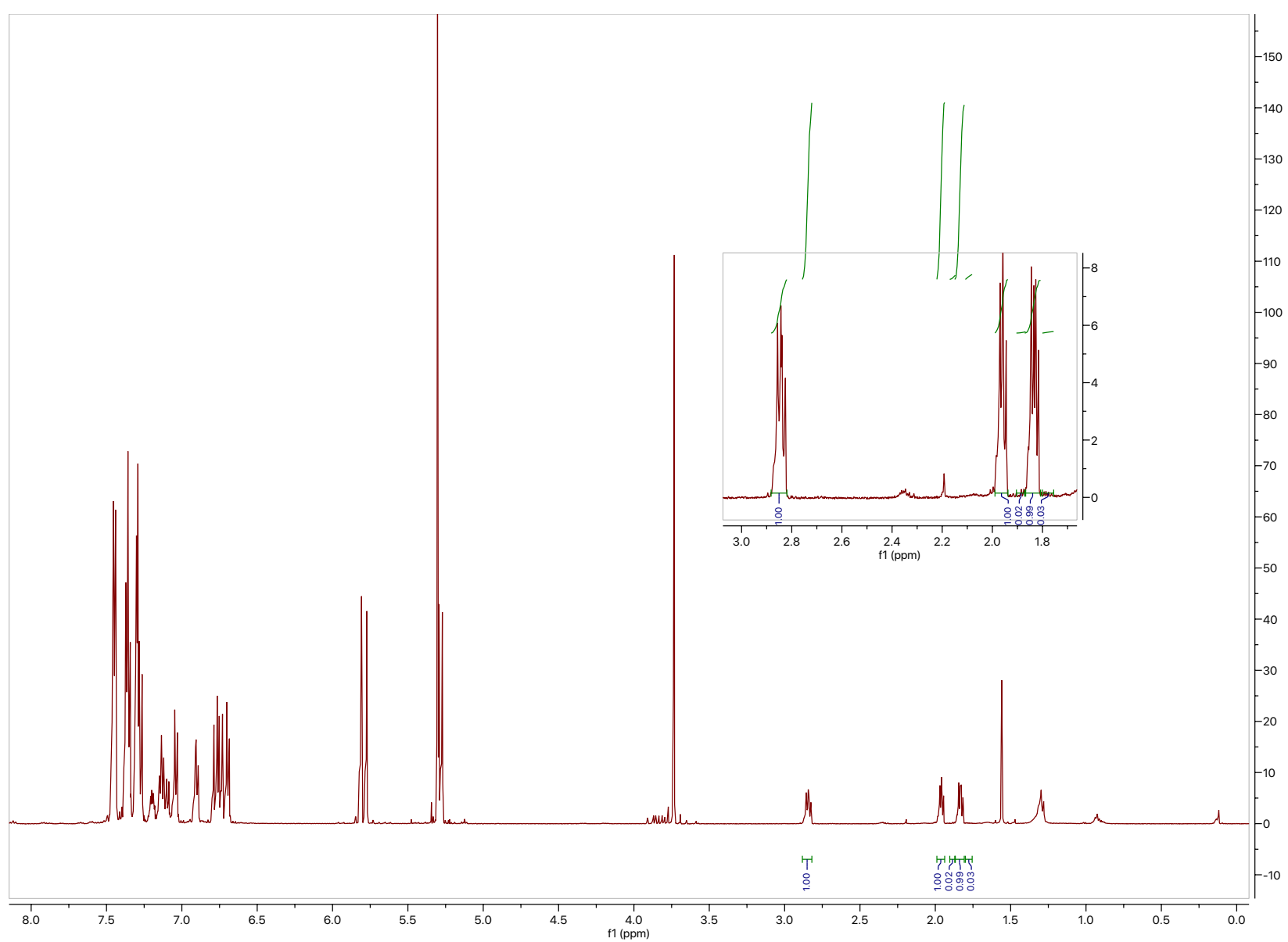


到

$8 c$

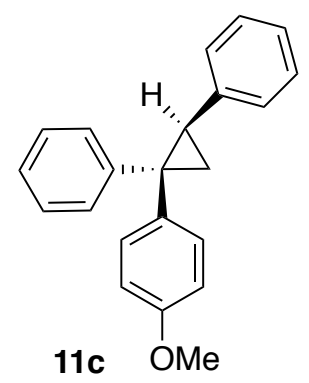

11c $\mathrm{OMe}$

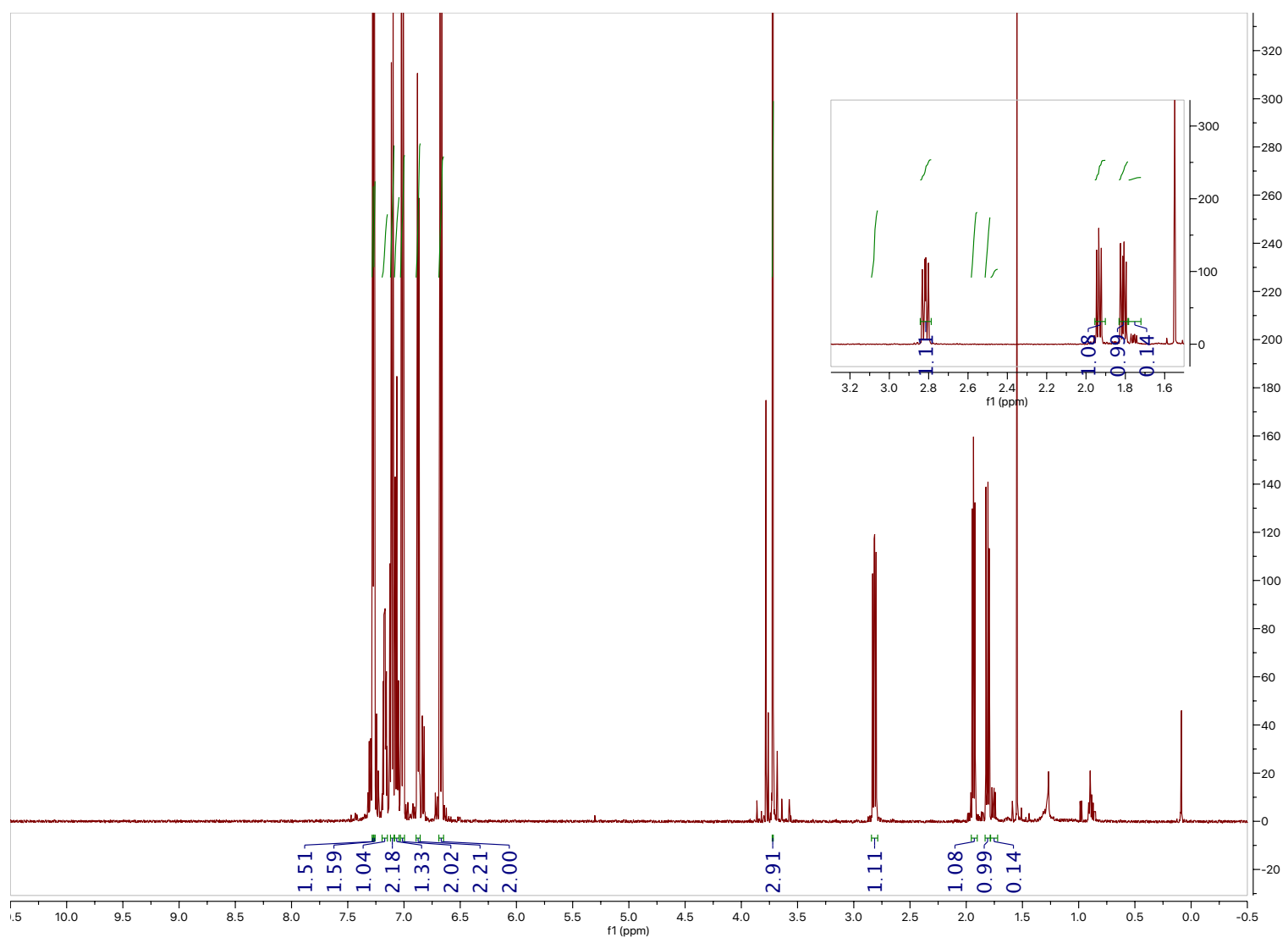


<smiles>C=Cc1ccccc1C=CC(=O)OCC</smiles>

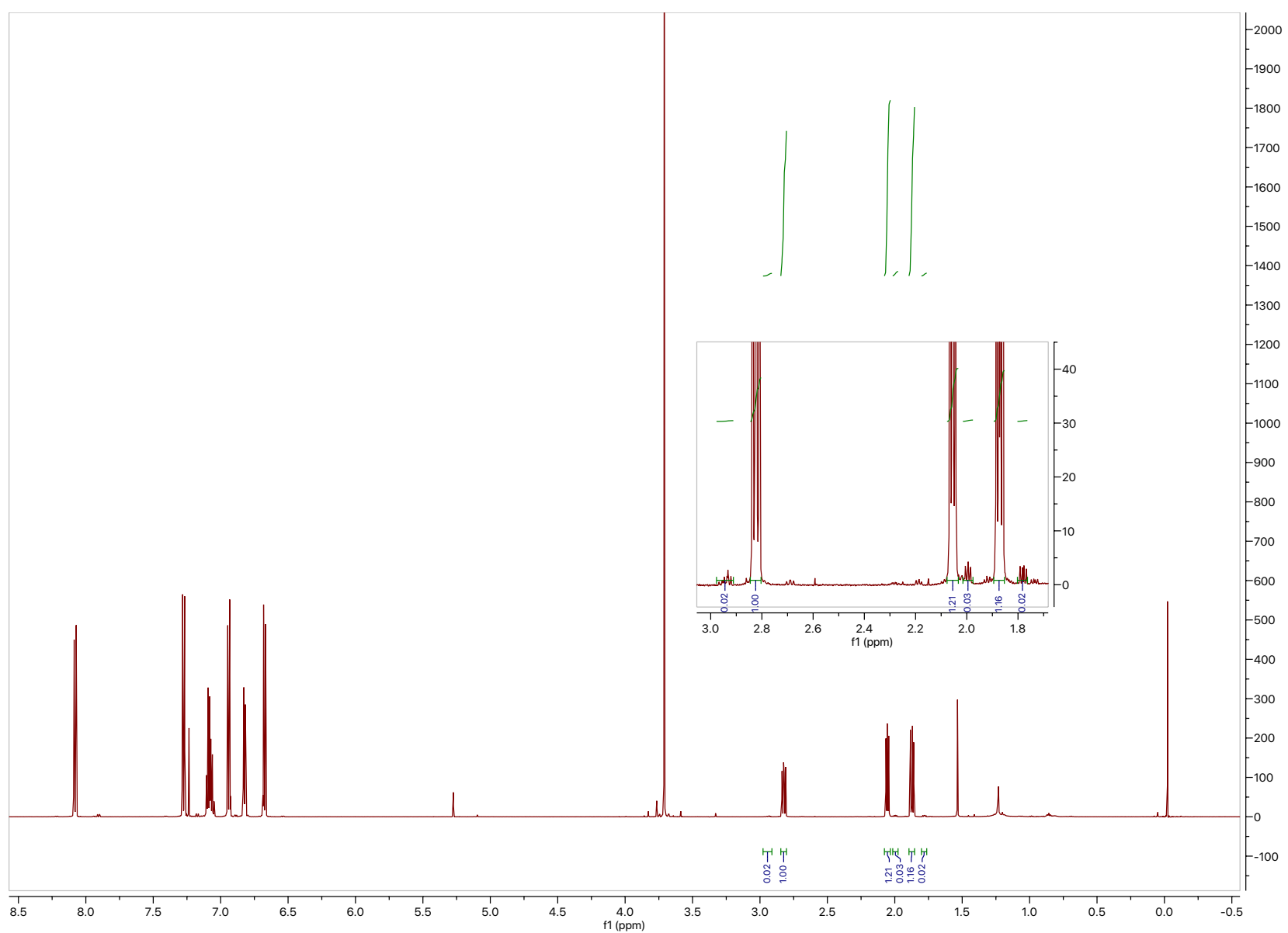


<smiles>C=Cc1ccccc1C=[Pb]Cc1ccc([N+](=O)[O-])cc1</smiles>

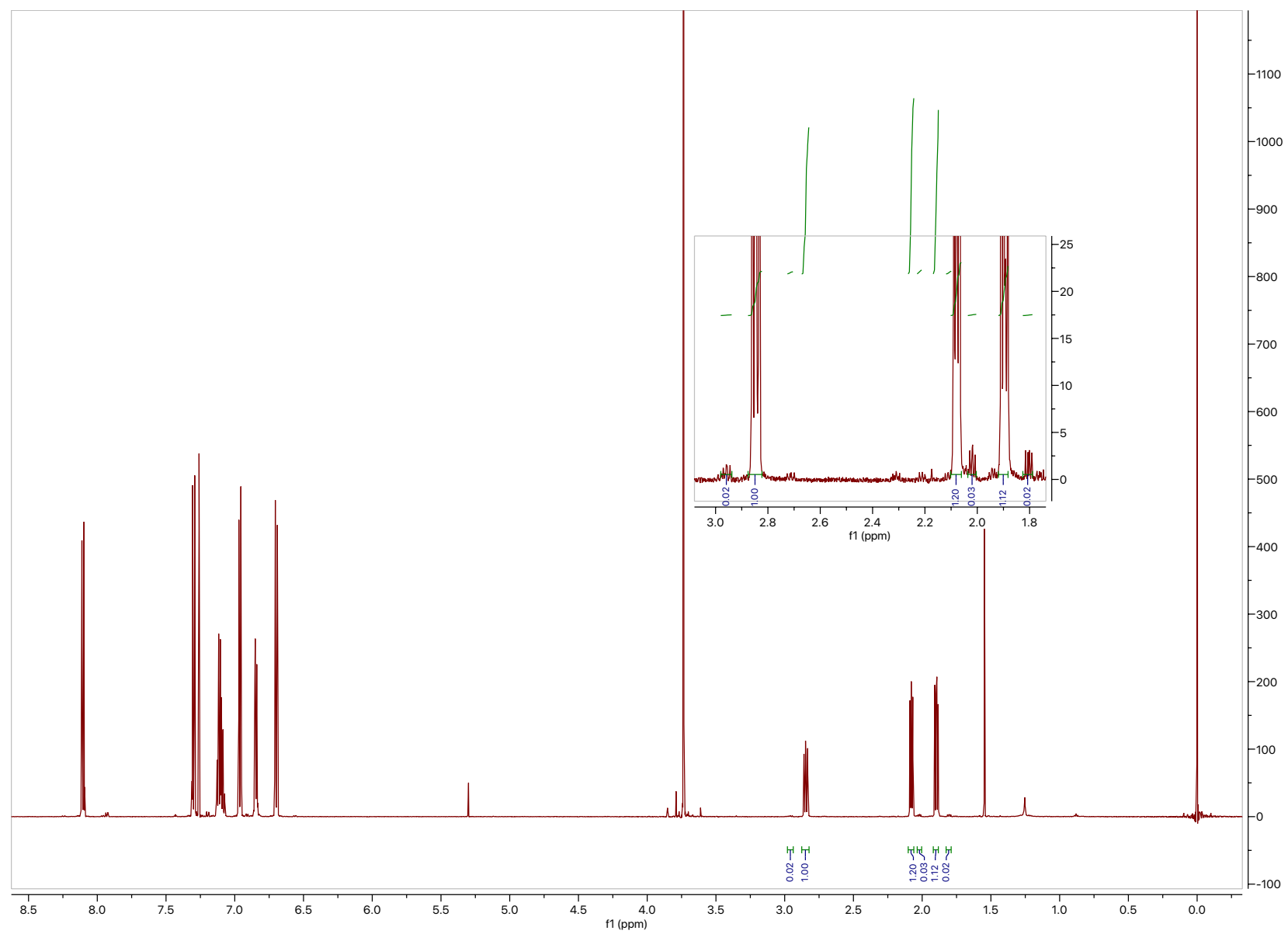


<smiles>COc1ccc(C(=N)c2ccc([N+](=O)[O-])cc2)cc1</smiles>

8d

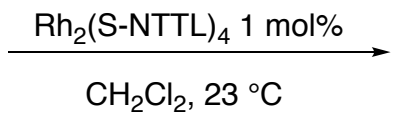

$\mathrm{CH}_{2} \mathrm{Cl}_{2}, 23^{\circ} \mathrm{C}$

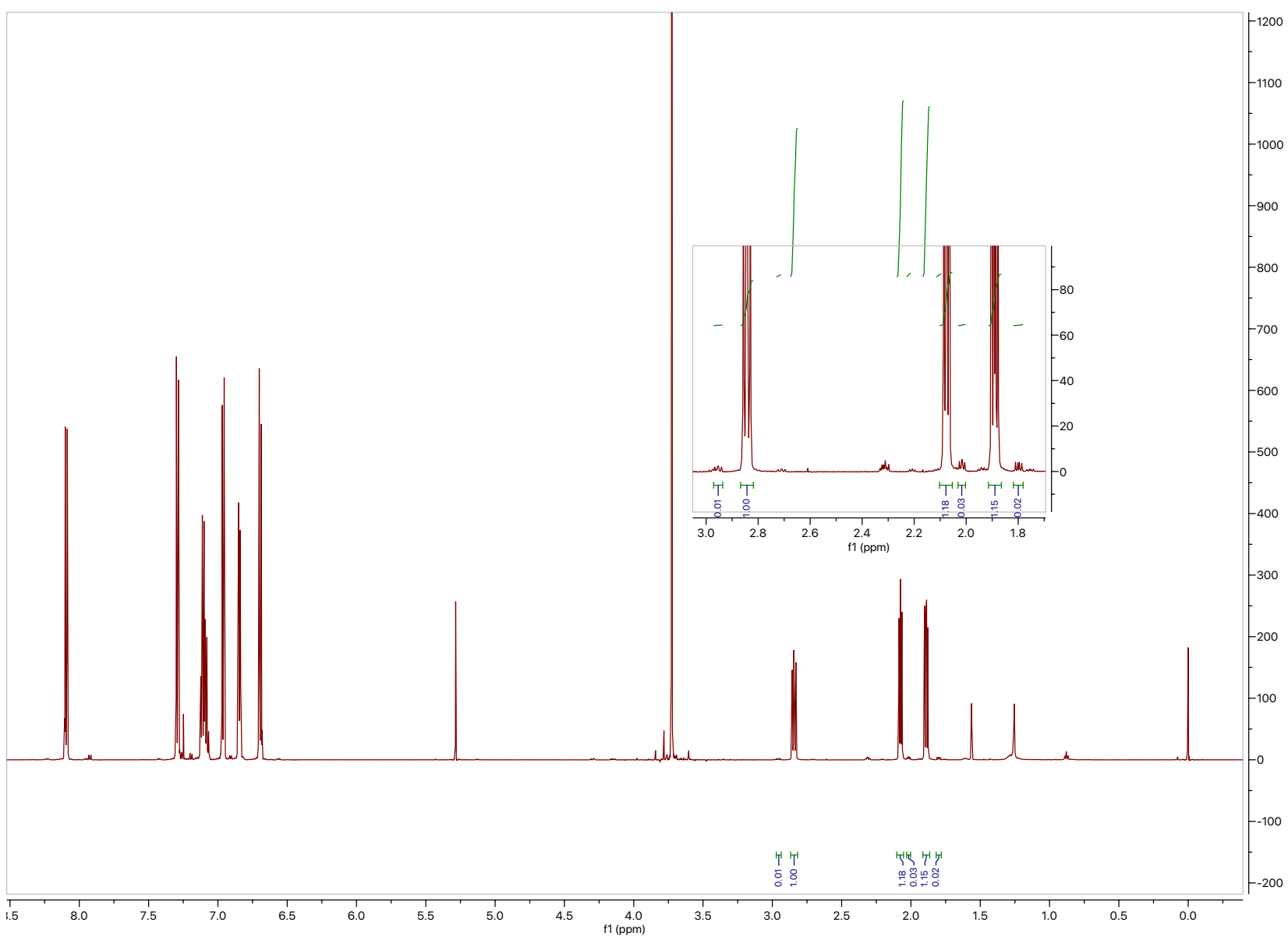

11d

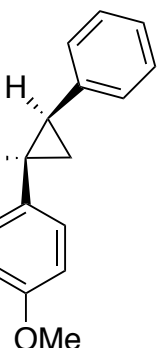


<smiles>N#Cc1ccccc1Cl</smiles>

$8 e$

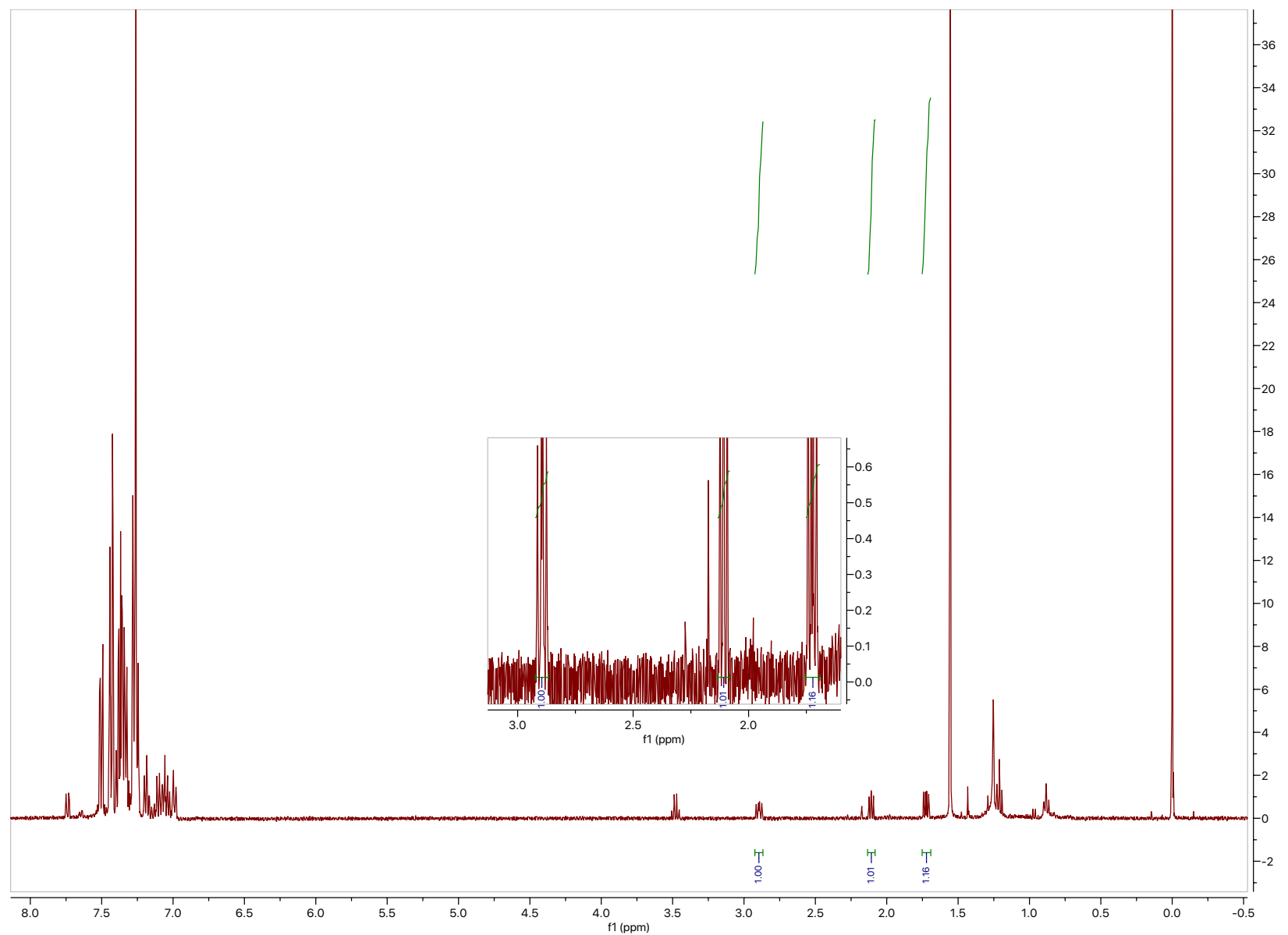




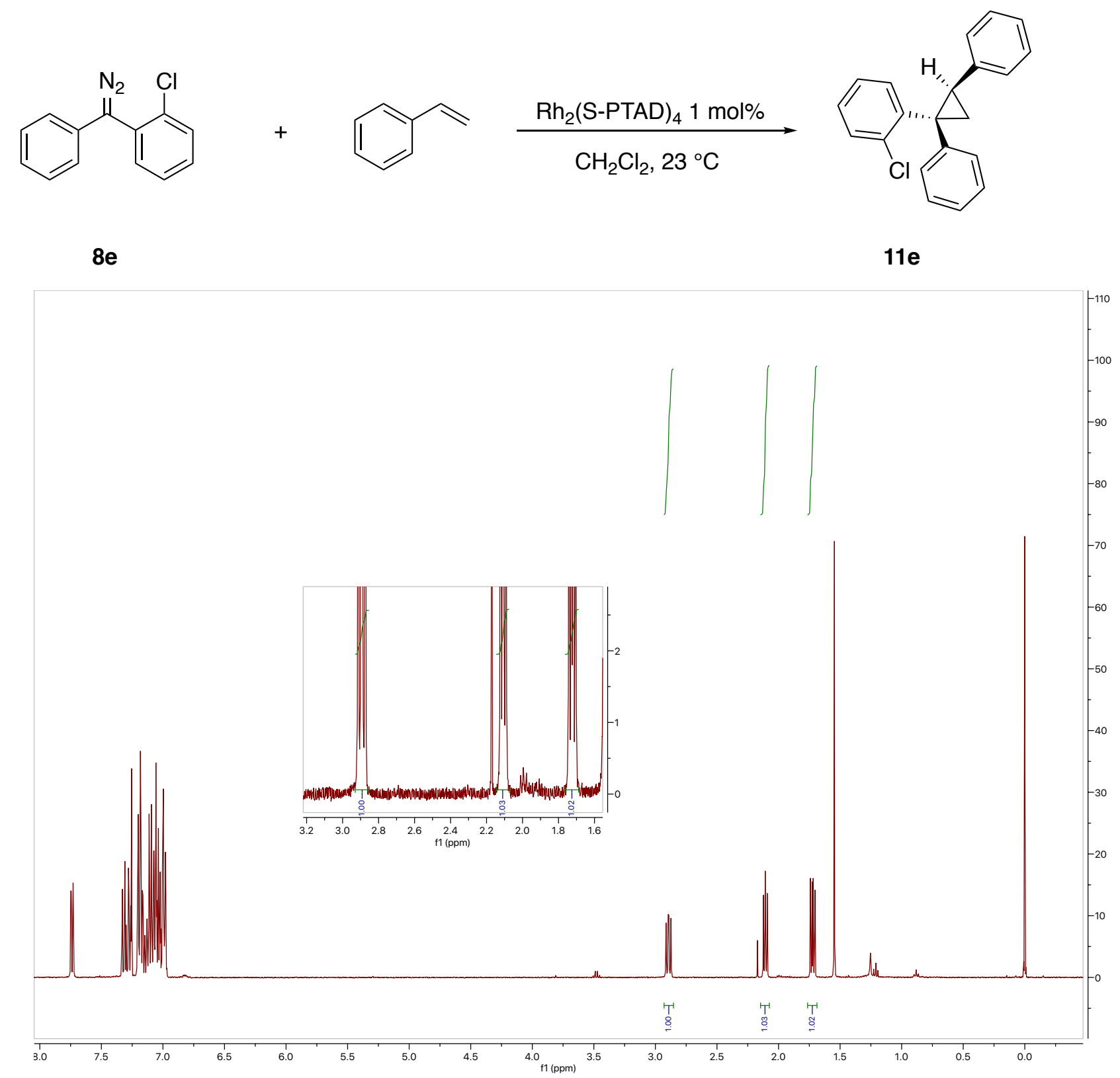


(1)

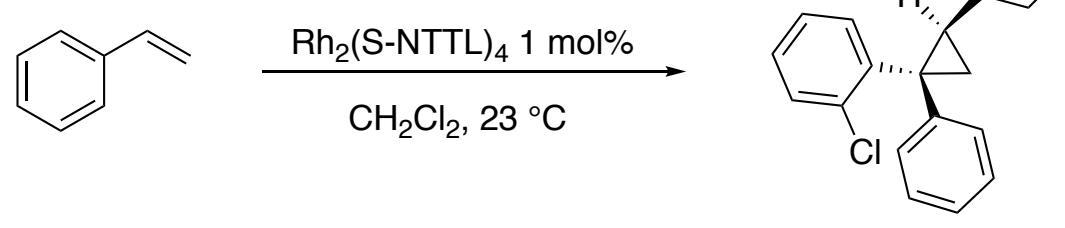

$11 \mathrm{e}$

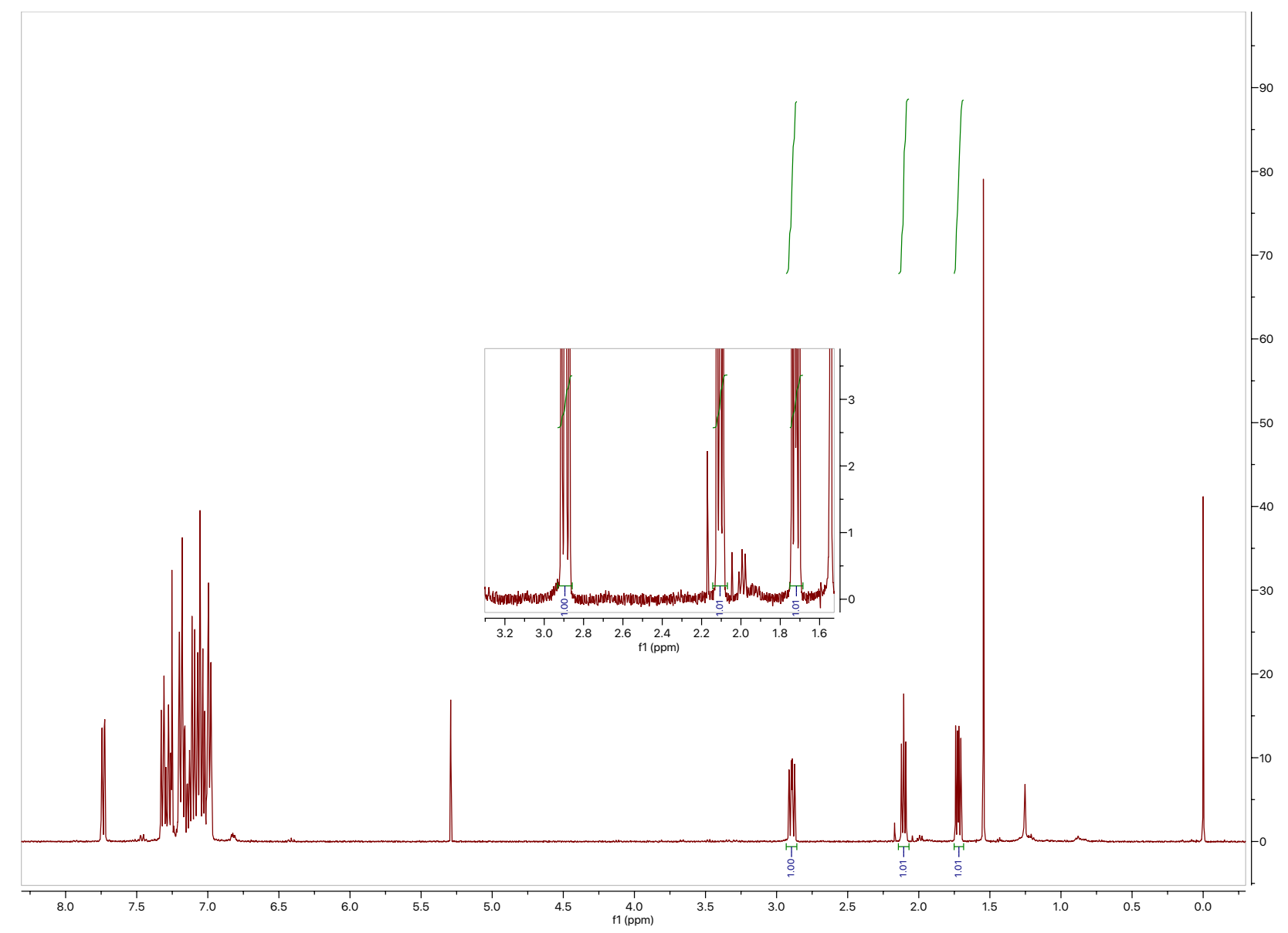


<smiles>N#Cc1ccc([N+](=O)[O-])cc1Cl</smiles>

$8 f$

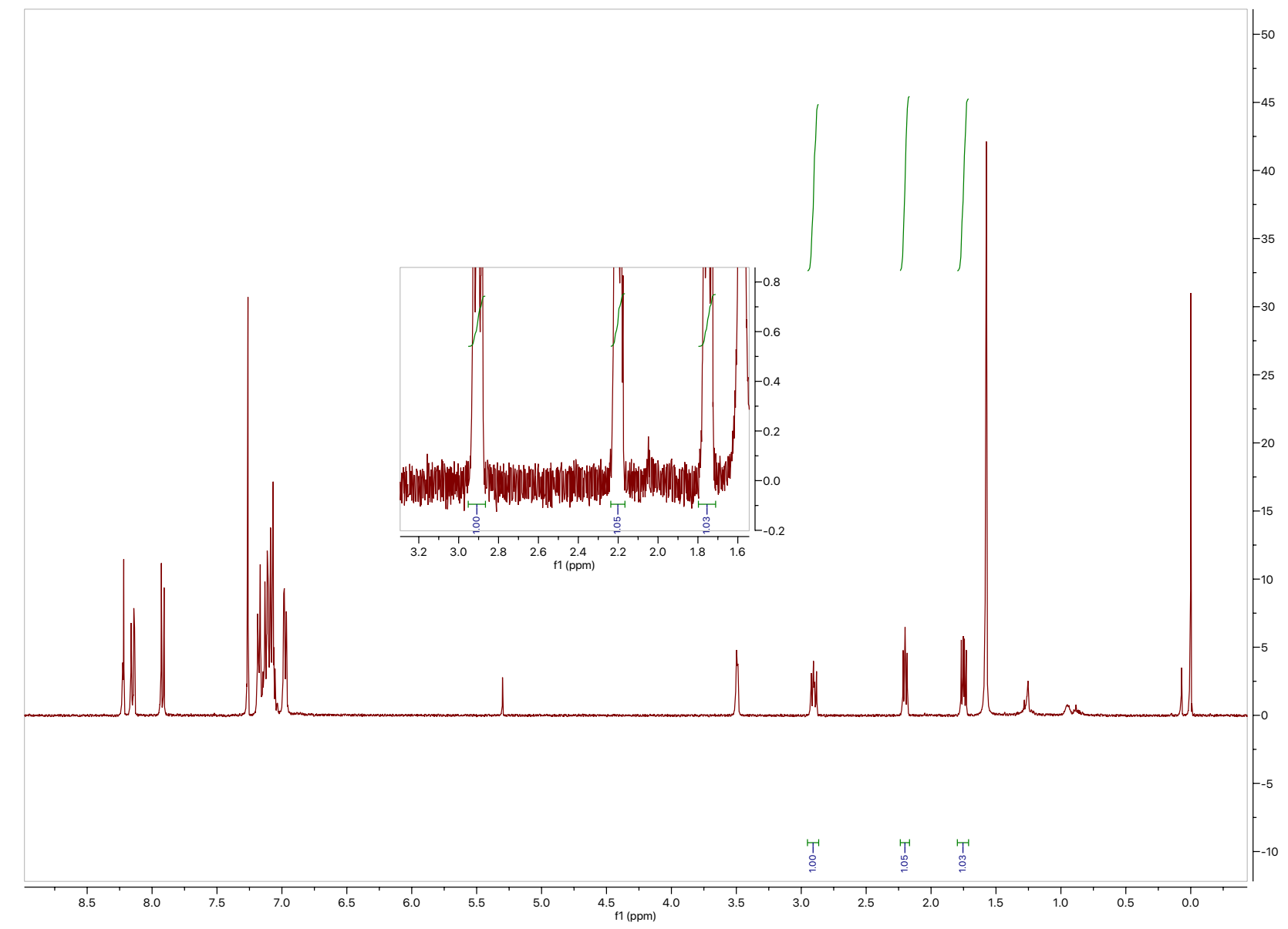




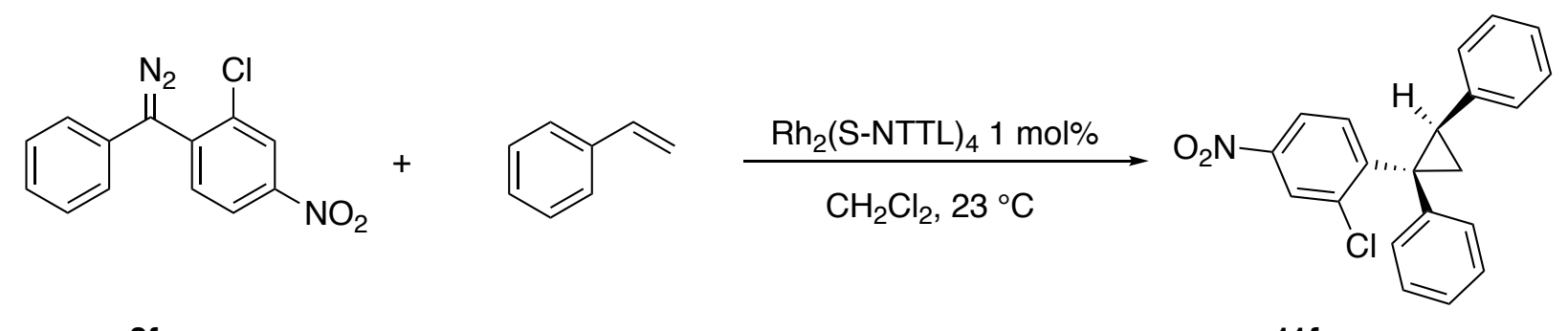

$8 f$

$11 f$

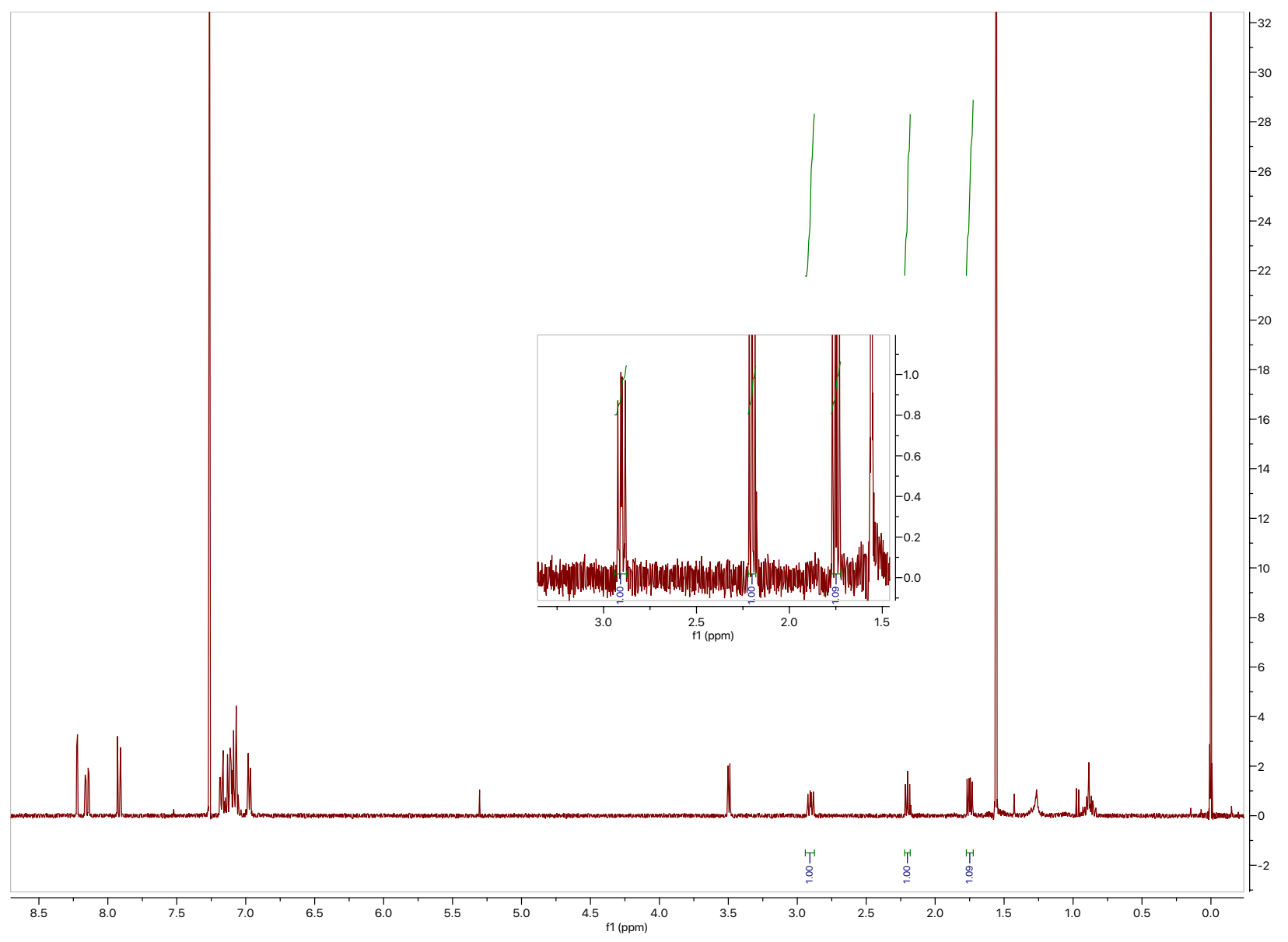




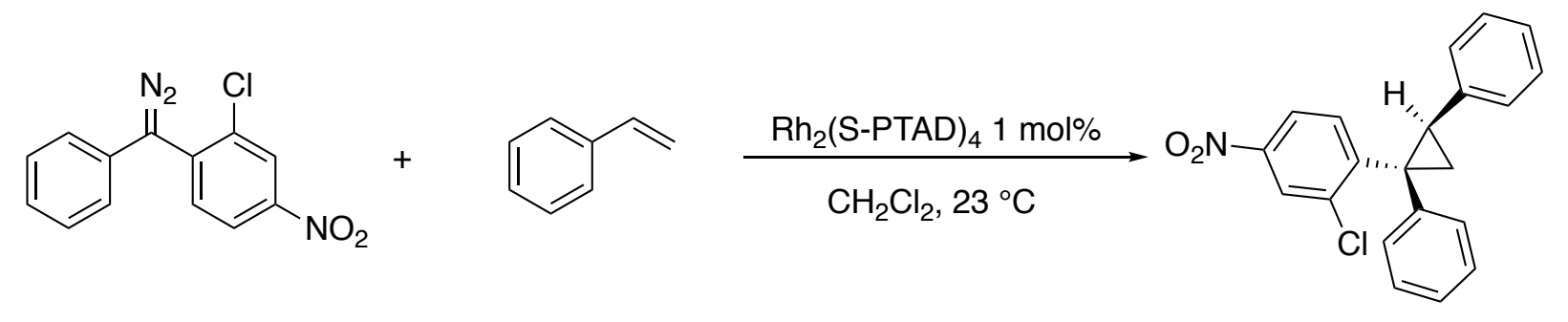

$8 f$

$11 f$

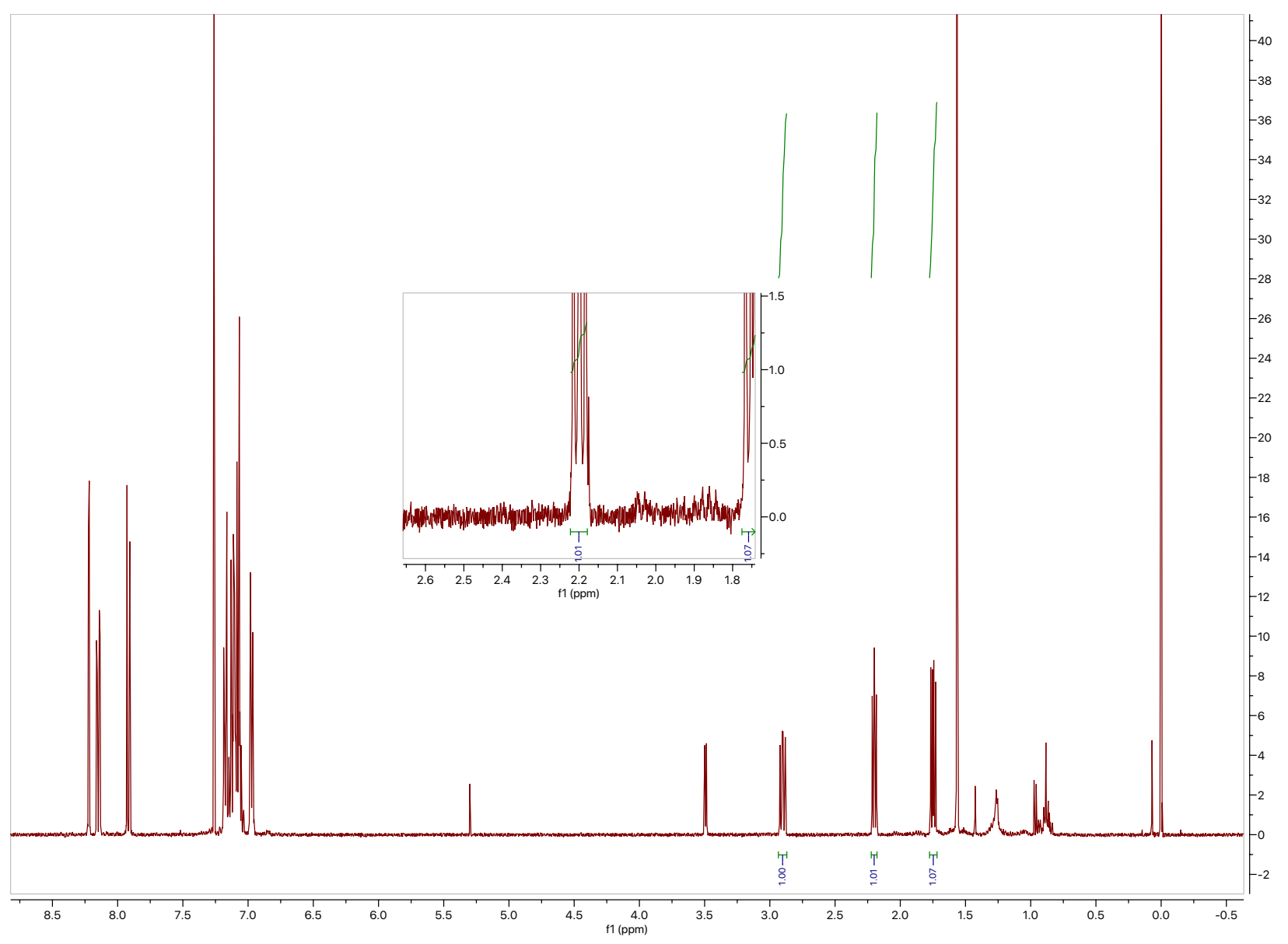




\section{HPLC Spectra}

事

$8 a$

DAD1 B, Sig=230,4 Ref=off (14-Oct-201_..ober-14-2019. 2019-10-15 09-25-491020-5-ml-eln-001-racbiphenyl.D)

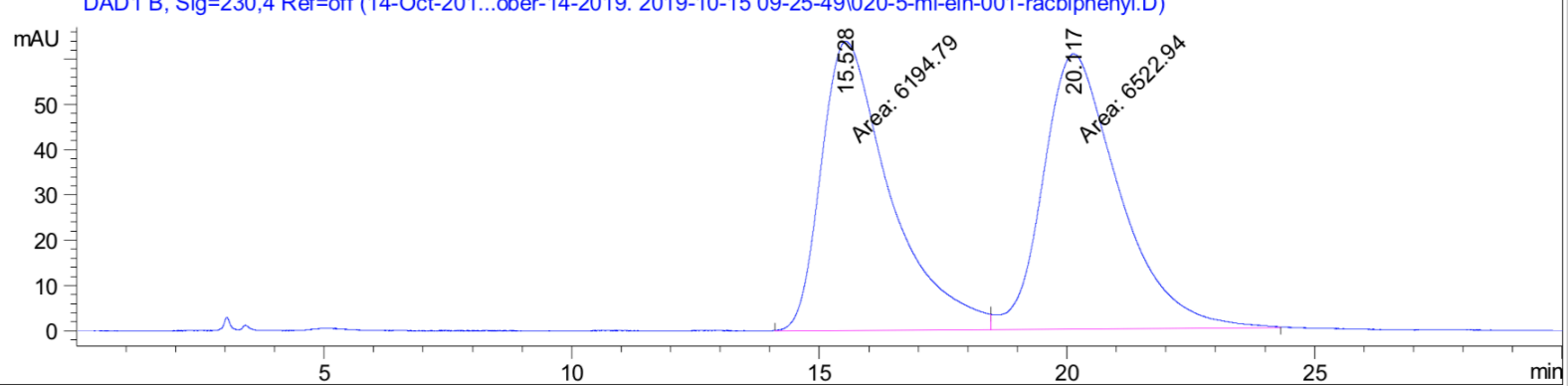

Signal 2: DAD1 B, Sig=230, 4 Ref=off

\begin{tabular}{|c|c|c|c|c|c|c|}
\hline $\begin{array}{c}\text { Peak } \\
\#\end{array}$ & $\begin{array}{c}\text { RetTime } \\
\text { [min] }\end{array}$ & Type & $\begin{array}{l}\text { Width } \\
\text { [min] }\end{array}$ & $\begin{array}{c}\text { Area } \\
{\left[\mathrm{mAU} U^{\star} \mathrm{S}\right]}\end{array}$ & $\begin{array}{l}\text { Height } \\
\text { [mAU] }\end{array}$ & $\begin{array}{c}\text { Area } \\
\frac{\circ}{0}\end{array}$ \\
\hline & & & & & ------ & - \\
\hline 1 & 15.528 & MF & 1.6156 & 6194.78613 & 63.90763 & 48.7098 \\
\hline 2 & 20.117 & FM & 1.7875 & 6522.94336 & 60.81913 & 51.2902 \\
\hline
\end{tabular}

Totals :

$1.27177 \mathrm{e} 4 \quad 124.72676$ 

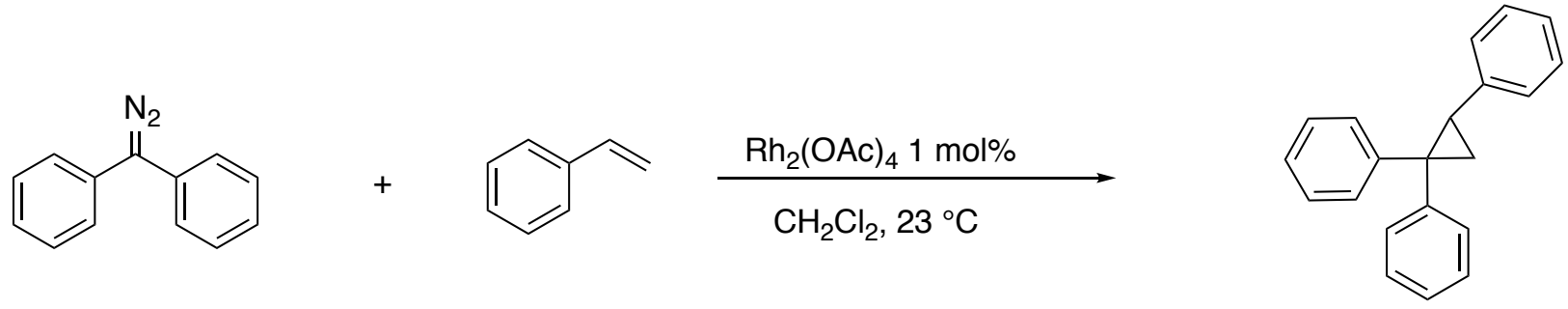

$8 a$

11a

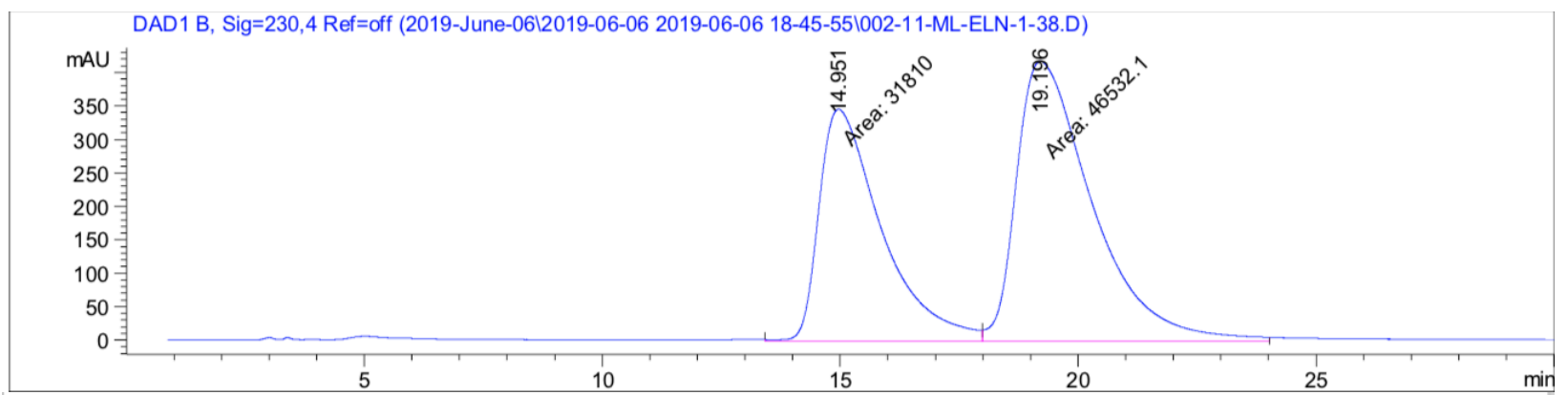

Signal 2: DAD1 B, Sig=230,4 Ref=off

\begin{tabular}{|c|c|c|c|c|c|c|}
\hline $\begin{array}{c}\text { Peak } \\
\#\end{array}$ & $\begin{array}{c}\text { RetTime } \\
\text { [min] }\end{array}$ & Type & $\begin{array}{l}\text { Width } \\
\text { [min] }\end{array}$ & $\begin{array}{c}\text { Area } \\
{\left[\mathrm{mAU}{ }^{\star} \mathrm{s}\right]}\end{array}$ & $\begin{array}{l}\text { Height } \\
\text { [mAU] }\end{array}$ & $\begin{array}{c}\text { Area } \\
\frac{\circ}{0}\end{array}$ \\
\hline \multicolumn{7}{|c|}{$----|-------|----|-------|----------|----------|-------$} \\
\hline 1 & 14.951 & $\mathrm{MF}$ & 1.5266 & $3.18100 \mathrm{e} 4$ & 347.29602 & 40.6040 \\
\hline 2 & 19.196 & FM & 1.8519 & $4.65321 e 4$ & 418.78320 & 59.3960 \\
\hline 7 & : & & & $7.83422 e 4$ & 766.07922 & \\
\hline
\end{tabular}



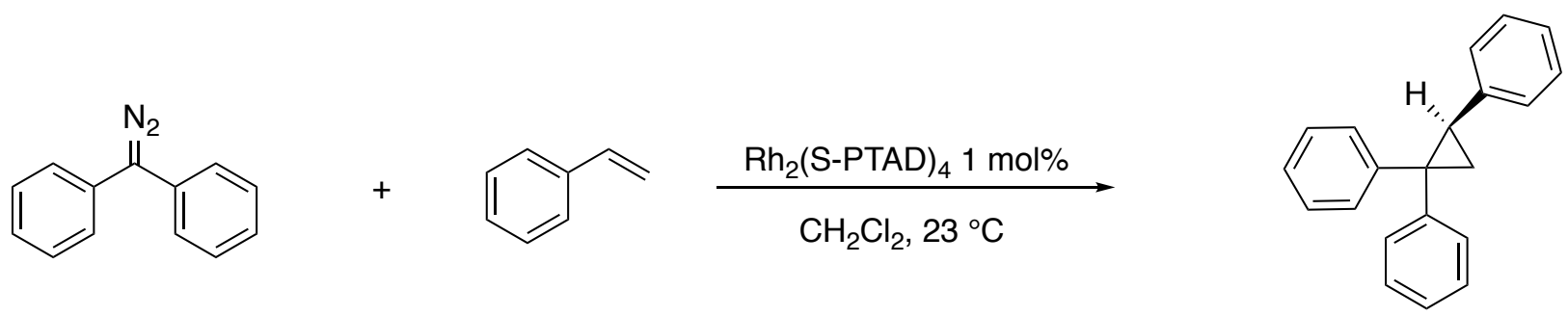

$8 a$

$11 a$

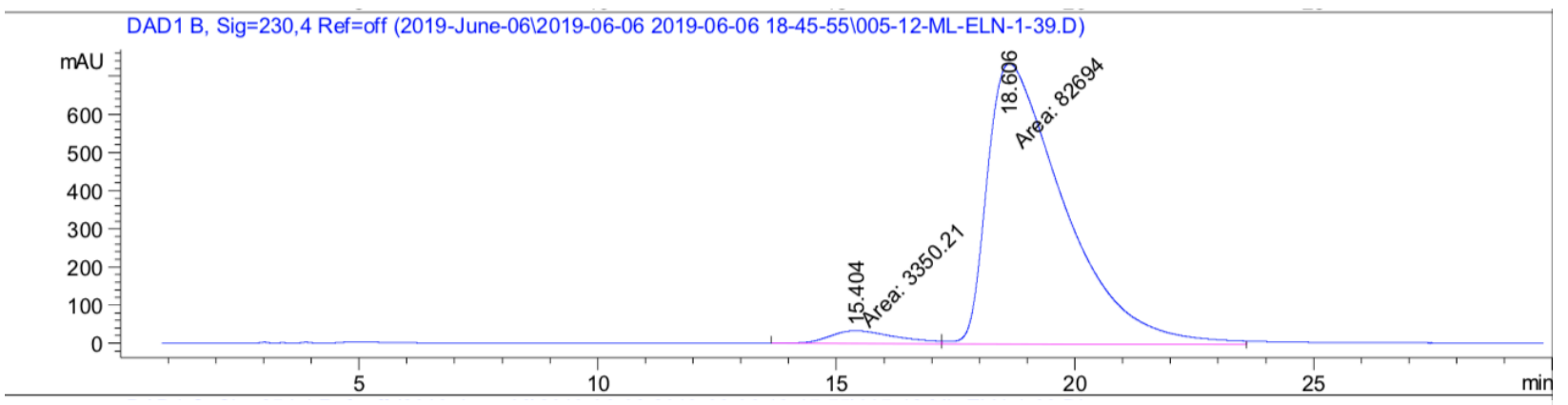

Signal 2: DAD1 B, Sig=230,4 Ref=off

\begin{tabular}{|c|c|c|c|c|c|c|}
\hline $\begin{array}{c}\text { Peak } \\
\quad \#\end{array}$ & $\begin{array}{c}\text { RetTime } \\
\text { [min] }\end{array}$ & Type & $\begin{array}{l}\text { Width } \\
\text { [min] }\end{array}$ & $\begin{array}{c}\text { Area } \\
{\left[\mathrm{mAU}{ }^{\star} \mathrm{s}\right]}\end{array}$ & $\begin{array}{l}\text { Height } \\
\text { [MAU] }\end{array}$ & $\begin{array}{c}\text { Area } \\
\quad \frac{\circ}{0}\end{array}$ \\
\hline---1 & $1-\ldots$ & -1 & $------\mid$ & |-------- & ------- & -----1 \\
\hline 1 & 15.404 & $\mathrm{MF}$ & 1.6434 & 3350.20605 & 33.97601 & 3.8936 \\
\hline 2 & 18.606 & FM & 1.8737 & $8.26940 \mathrm{e} 4$ & 735.56940 & 96.1064 \\
\hline Tota & & & & $8.60442 \mathrm{e} 4$ & 769.54541 & \\
\hline
\end{tabular}



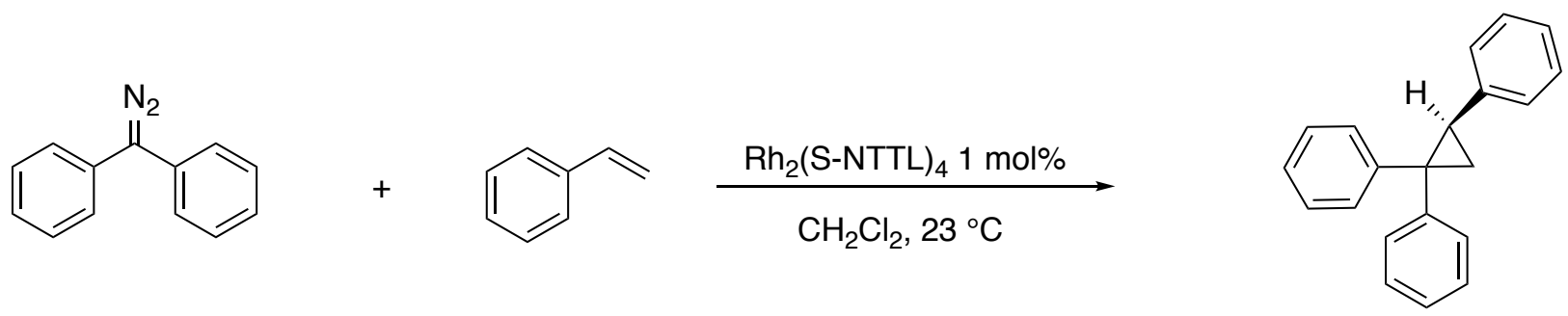

$8 a$

11a

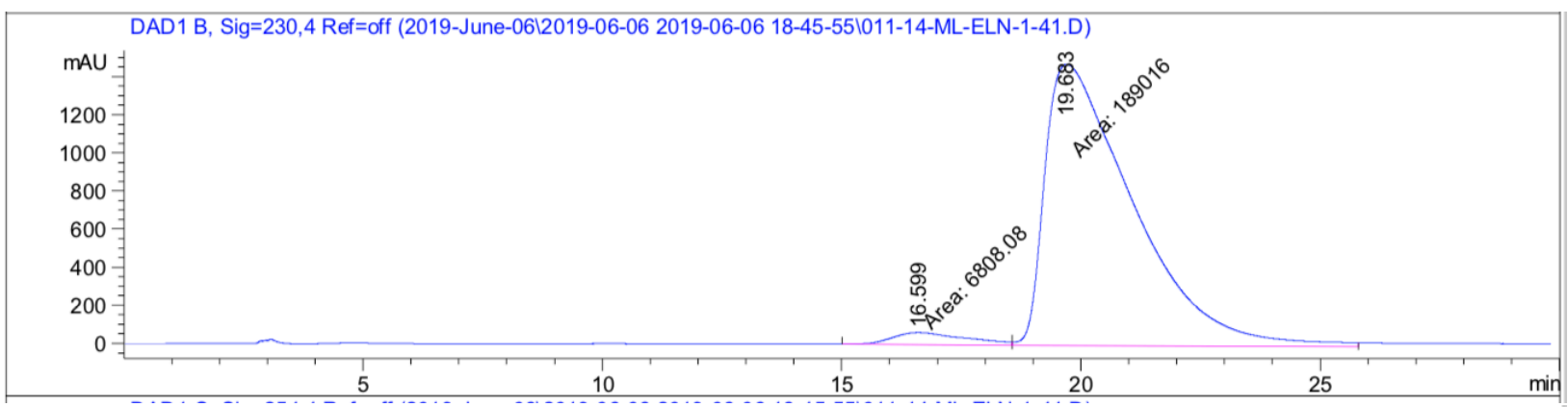

Signal 2: DAD1 B, Sig=230, 4 Ref=off

\begin{tabular}{|c|c|c|c|c|c|c|}
\hline $\begin{array}{c}\text { Peak } \\
\#\end{array}$ & $\begin{array}{c}\text { RetTime } \\
\text { [min] }\end{array}$ & Type & $\begin{array}{l}\text { Width } \\
\text { [min] }\end{array}$ & $\begin{array}{c}\text { Area } \\
{\left[\mathrm{mAU}{ }^{\star} \mathrm{s}\right]}\end{array}$ & $\begin{array}{l}\text { Height } \\
{[\mathrm{mAU}]}\end{array}$ & $\begin{array}{c}\text { Area } \\
\frac{\circ}{0}\end{array}$ \\
\hline & & & & & & \\
\hline 1 & & & & 08057 & 25 & 66 \\
\hline 0 & 83 & & 48 & $1.89016 \mathrm{e} 5$ & 1475.68250 & \\
\hline
\end{tabular}

Totals : $\quad 1.95824 \mathrm{e} 5 \quad 1539.38674$ 
<smiles>N#CC(c1ccccc1)c1ccc([N+](=O)[O-])cc1</smiles>

$8 b$<smiles>C=Cc1ccccc1</smiles>

$\mathrm{Rh}_{2}(\mathrm{OAc})_{4} 1 \mathrm{~mol} \%$

$\mathrm{CH}_{2} \mathrm{Cl}_{2}, 23^{\circ} \mathrm{C}$<smiles>O=[N+]([O-])c1ccccc1</smiles>

$H$

5

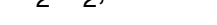

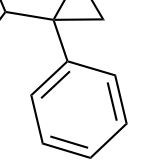

11b

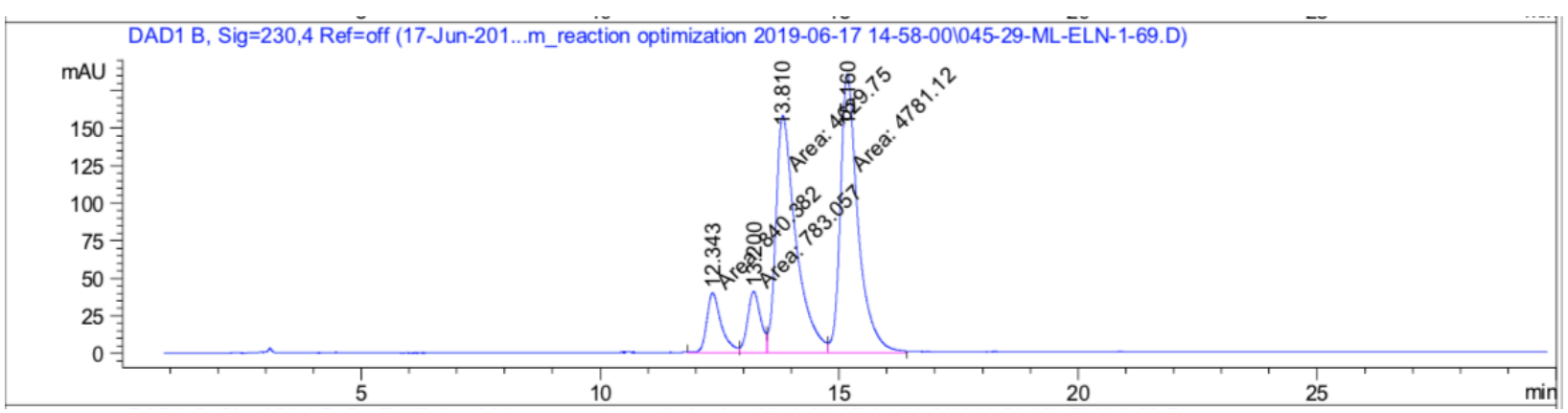

Signal 2: DAD1 B, Sig=230,4 Ref=off

\begin{tabular}{|c|c|c|c|c|c|c|}
\hline $\begin{array}{c}\text { Peak } \\
\quad \#\end{array}$ & $\begin{array}{c}\text { RetTime } \\
\text { [min] }\end{array}$ & Type & $\begin{array}{l}\text { Width } \\
\text { [min] }\end{array}$ & $\begin{array}{c}\text { Area } \\
{\left[\mathrm{mAU}^{\star} \mathrm{S}\right]}\end{array}$ & $\begin{array}{l}\text { Height } \\
\text { [mAU] }\end{array}$ & $\begin{array}{c}\text { Area } \\
\quad \frac{\circ}{0}\end{array}$ \\
\hline \multicolumn{7}{|c|}{ 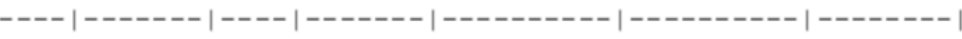 } \\
\hline 1 & 12.343 & MF & 0.3525 & 840.38214 & 39.73832 & 7.6161 \\
\hline 2 & 13.200 & MF & 0.3202 & 783.05688 & 40.76136 & 0966 \\
\hline 3 & 13.810 & MF & 0.4890 & 4629.74609 & 157.79266 & 41.9578 \\
\hline 4 & 15.160 & FM & 0.4287 & 4781.11523 & 185.89740 & 43.3296 \\
\hline
\end{tabular}

Totals : $\quad 1.10343 e 4 \quad 424.18975$ 
<smiles>N#CC(c1ccccc1)c1ccc([N+](=O)[O-])cc1</smiles>

$8 b$
$\mathrm{Rh}_{2}(\mathrm{~S}-\mathrm{NTTL})_{4} 1 \mathrm{~mol} \%$ $\mathrm{CH}_{2} \mathrm{Cl}_{2}, 23^{\circ} \mathrm{C}$

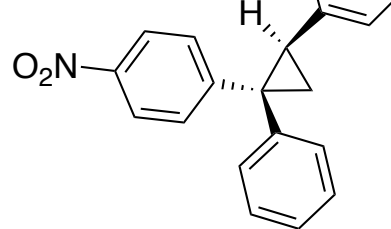

11b DAD1 B, Sig=230,4 Ref=off (Snapshot.d)

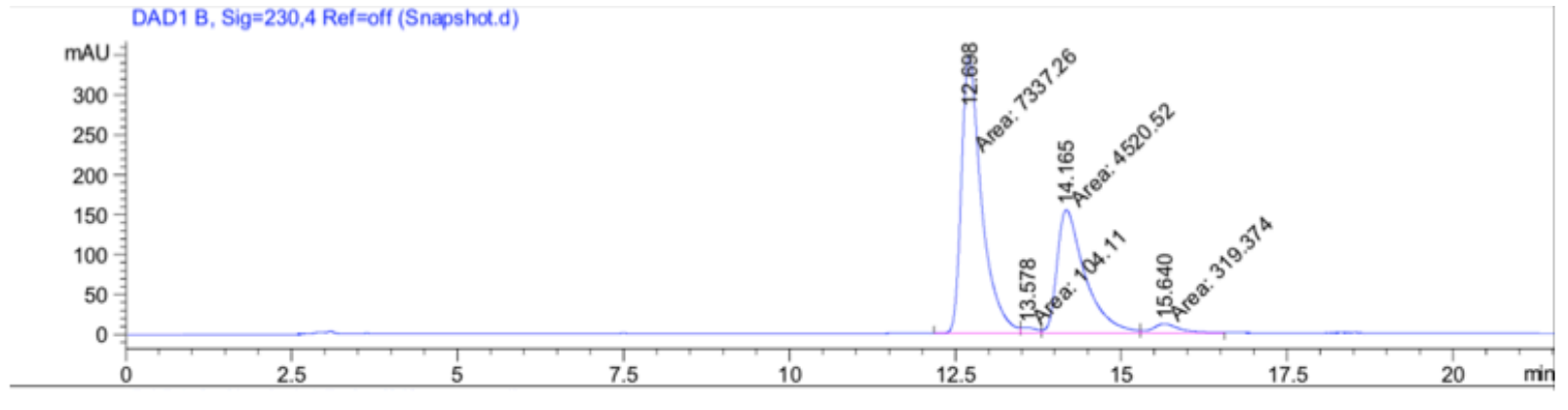

Signal 2: DAD1 B, Sig=230, 4 Ref=of $f$

\begin{tabular}{|c|c|c|c|c|c|c|}
\hline $\begin{array}{c}\text { Peak } 1 \\
\#\end{array}$ & $\begin{array}{l}\text { RetTime } \\
\text { [min] }\end{array}$ & Type & $\begin{array}{c}\text { Width } \\
\text { [min] }\end{array}$ & $\begin{array}{c}\text { Area } \\
{\left[m A U^{*} s\right]}\end{array}$ & $\begin{array}{l}\text { Height } \\
\text { [mAU] }\end{array}$ & $\begin{array}{c}\text { Area } \\
\frac{9}{6}\end{array}$ \\
\hline \multicolumn{7}{|c|}{ 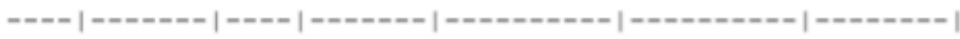 } \\
\hline 1 & 12.698 & MF & 0.3510 & 7337.25732 & 348.34833 & 59.7435 \\
\hline 2 & 13.578 & MF & 0.2654 & 104.11029 & 6.53808 & 0.8477 \\
\hline 3 & 14.165 & MF & 0.4891 & 4520.51709 & 154.05446 & 36.8083 \\
\hline 4 & 15.640 & FM & 0.4610 & 319.37375 & 11.54610 & 2.6005 \\
\hline 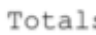 & & & & $1.22813 \mathrm{e} 4$ & .48697 & \\
\hline
\end{tabular}


<smiles></smiles>

$8 b$<smiles>C=Cc1ccccc1</smiles>

$\mathrm{Rh}_{2}(\mathrm{~S}-\mathrm{PTAD})_{4} 1 \mathrm{~mol} \%$ $\mathrm{CH}_{2} \mathrm{Cl}_{2}, 23^{\circ} \mathrm{C}$

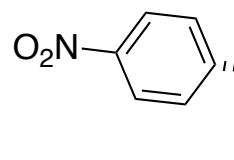

$\mathrm{H}$

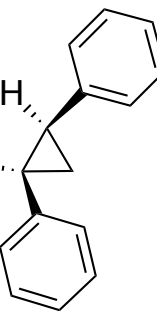

11b

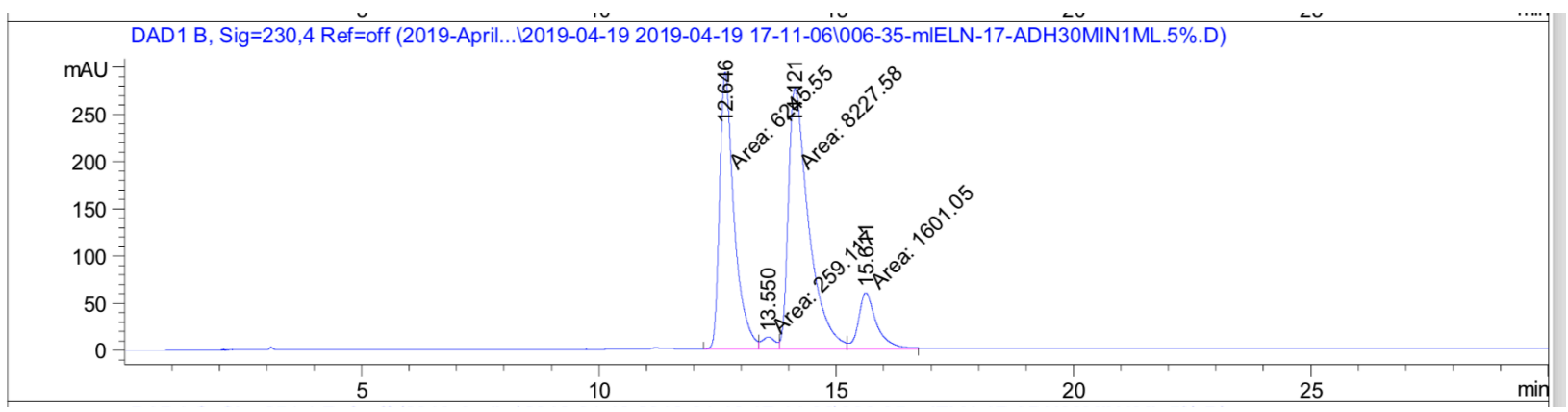

\begin{tabular}{|c|c|c|c|c|c|c|}
\hline $\begin{array}{c}\text { Peak } \\
\#\end{array}$ & $\begin{array}{c}\text { RetTime } \\
\text { [min] }\end{array}$ & Type & $\begin{array}{l}\text { Width } \\
{[\mathrm{min}]}\end{array}$ & $\begin{array}{c}\text { Area } \\
{\left[m A U^{*} s\right]}\end{array}$ & $\begin{array}{l}\text { Height } \\
{[\mathrm{mAU}]}\end{array}$ & $\begin{array}{c}\text { Area } \\
\%\end{array}$ \\
\hline & & & & & & -------- \\
\hline 1 & 12.646 & $\mathrm{MF}$ & 0.3549 & 6245.55225 & 293.26422 & 38.2382 \\
\hline 2 & 13.550 & MF & 0.3376 & 259.11688 & 12.79360 & 1.5864 \\
\hline 3 & 14.121 & MF & 0.4968 & 8227.58008 & 276.01035 & 50.3730 \\
\hline 4 & 15.611 & FM & 0.4466 & 1601.05176 & 59.75366 & 9.8024 \\
\hline
\end{tabular}

Totals :

$1.63333 e 4 \quad 641.82182$ 
<smiles></smiles>

$8 b$<smiles>C=Cc1ccccc1</smiles>

$\mathrm{Rh}_{2}(\mathrm{~S}-\mathrm{DOSP})_{4} 1 \mathrm{~mol} \%$

$\mathrm{CH}_{2} \mathrm{Cl}_{2}, 23^{\circ} \mathrm{C}$<smiles>O=[N+]([O-])c1ccccc1</smiles>

$\mathrm{H}$

$\mathrm{H}$,<smiles>C/C=C\CCc1ccccc1</smiles>

$11 b$

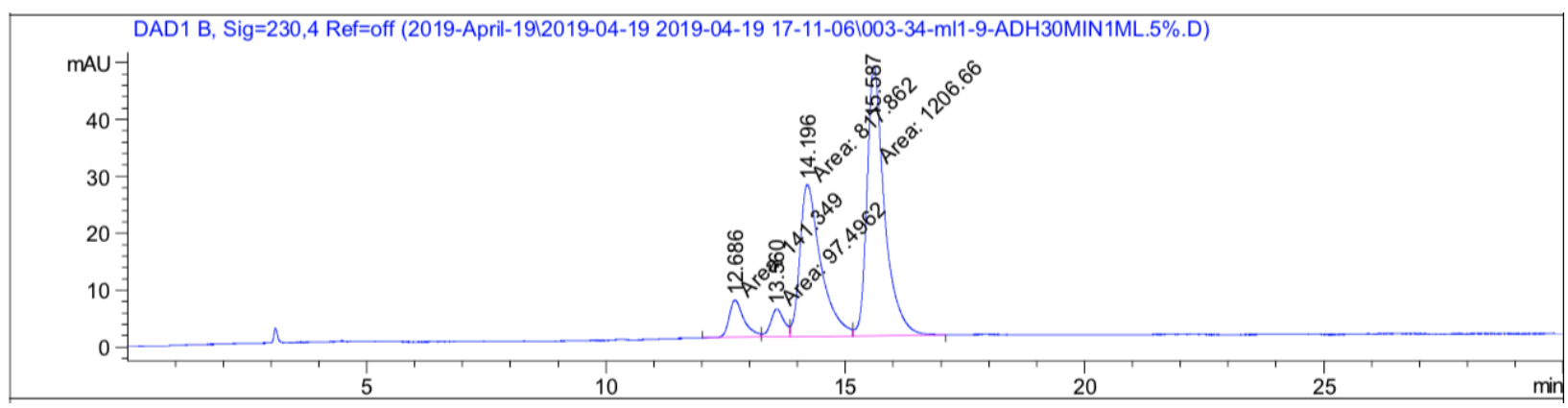

Signal 2: DAD1 B, Sig=230, 4 Ref=off

\begin{tabular}{|c|c|c|c|c|c|c|}
\hline $\begin{array}{c}\text { Peak } \\
\#\end{array}$ & $\begin{array}{c}\text { RetTime } \\
\text { [min] }\end{array}$ & Type & $\begin{array}{l}\text { Width } \\
\text { [min] }\end{array}$ & $\begin{array}{c}\text { Area } \\
{\left[\mathrm{mAU}^{\star} \mathrm{s}\right]}\end{array}$ & $\begin{array}{l}\text { Height } \\
{[\mathrm{mAU}]}\end{array}$ & $\begin{array}{c}\text { Area } \\
\frac{\circ}{0}\end{array}$ \\
\hline \multicolumn{7}{|c|}{$----|-------|----|-------|----------|----------|--------\mid$} \\
\hline 1 & 12.686 & $\mathrm{MF}$ & 0.3578 & 141.34885 & 6.58496 & 6.2451 \\
\hline 2 & 13.560 & MF & 0.3323 & 97.49619 & 4.88990 & 4.3076 \\
\hline 3 & 14.196 & $\mathrm{MF}$ & 0.5095 & 817.86188 & 26.75207 & 36.1348 \\
\hline 4 & 15.587 & FM & 0.4244 & 1206.65723 & 47.38936 & 53.3126 \\
\hline$a$ & 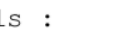 & & & 2263.36414 & 61630 & \\
\hline
\end{tabular}


<smiles>C=Cc1ccccc1</smiles>

8c

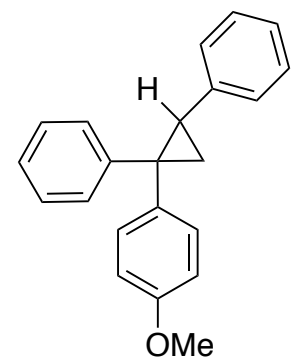

11c

DAD1 B, Sig=230,4 Ref=off (14-Oct-2019lOctober-14-2019. 2019-10-15 09-25-491011-65-ML-ELN-001-191.D)

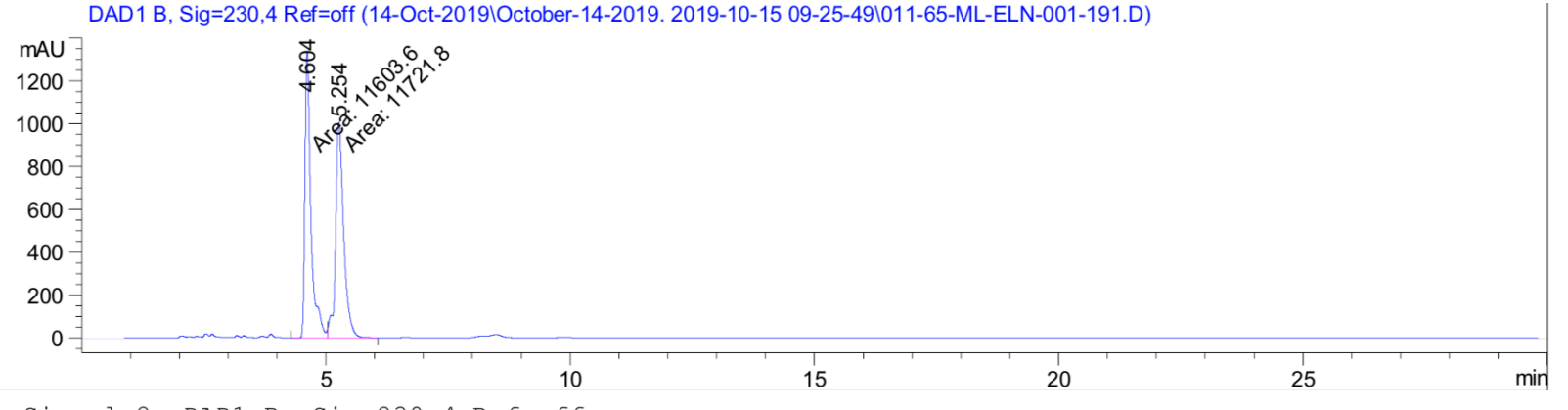

Signal 2: DAD1 B, Sig=230,4 Ref=off

\begin{tabular}{|c|c|c|c|c|c|c|}
\hline $\begin{array}{c}\text { Peak } \\
\quad \#\end{array}$ & $\begin{array}{c}\text { RetTime } \\
\text { [min] }\end{array}$ & Type & $\begin{array}{l}\text { Width } \\
\text { [min] }\end{array}$ & $\begin{array}{c}\text { Area } \\
{\left[\mathrm{mAU}^{*} \mathrm{~S}\right]}\end{array}$ & $\begin{array}{l}\text { Height } \\
\text { [mAU] }\end{array}$ & $\begin{array}{c}\text { Area } \\
\frac{\circ}{0}\end{array}$ \\
\hline \multicolumn{7}{|c|}{$----|-------|----|-------|----------|----------|--------$} \\
\hline 1 & 4.604 & $\mathrm{MF}$ & 0.1449 & $1.16036 \mathrm{e} 4$ & 1334.61938 & 49.7466 \\
\hline 2 & 5.254 & FM & 0.1944 & $1.17218 \mathrm{e} 4$ & 1005.07471 & 50.2534 \\
\hline & & & & $2.33254 e 4$ & .69409 & \\
\hline
\end{tabular}


<smiles>C=Cc1ccccc1</smiles><smiles>COc1ccc([C@]2(c3ccccc3)C[C@H]2c2ccccc2)cc1</smiles>

$8 c$

11c OMe

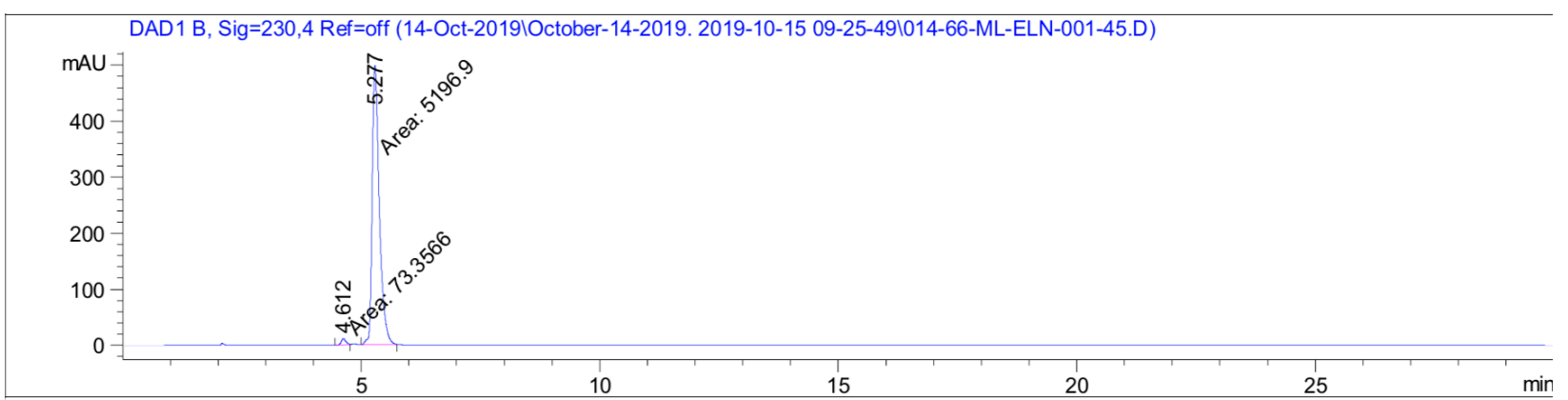

Signal 2: DAD1 B, Sig=230,4 Ref=off

\begin{tabular}{|c|c|c|c|c|c|c|}
\hline $\begin{array}{c}\text { Peak } \\
\quad \#\end{array}$ & $\begin{array}{c}\text { RetTime } \\
\text { [min] }\end{array}$ & Type & $\begin{array}{l}\text { Width } \\
\text { [min] }\end{array}$ & $\begin{array}{c}\text { Area } \\
{\left[\mathrm{mAU}^{*} \mathrm{~s}\right]}\end{array}$ & $\begin{array}{l}\text { Height } \\
\text { [mAU] }\end{array}$ & $\begin{array}{c}\text { Area } \\
\frac{\circ}{0}\end{array}$ \\
\hline- & -1 & & - & ----------- & - & -------1 \\
\hline 1 & 4.612 & MM & 0.1117 & 73.35658 & 10.94165 & 1.3919 \\
\hline 2 & 5.277 & MM & 0.1738 & 5196.89990 & 498.45493 & 98.6081 \\
\hline
\end{tabular}

Totals :

$5270.25648 \quad 509.39657$ 
<smiles>COc1ccc(C=N)cc1</smiles><smiles>COc1ccc([C@@H]2C[C@H]2c2ccccc2)cc1</smiles>

$8 c$

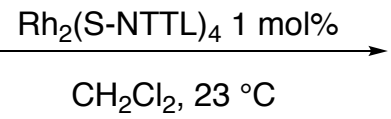

DAD1 B, Sig=230,4 Ref=off (14-Oct-2019lOctober-14-2019. 2019-10-15 09-25-491017-67-ML-ELN-001-46.D)

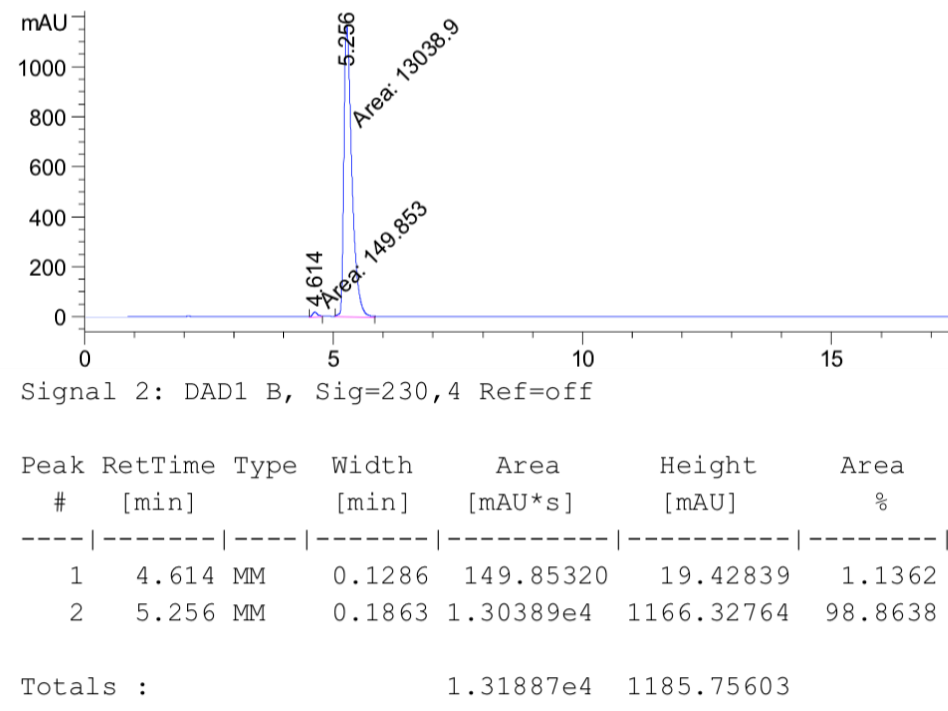




8c

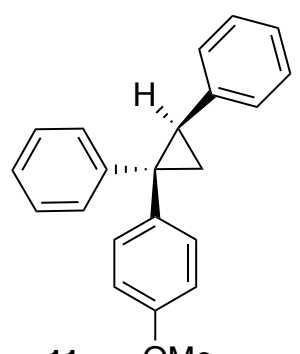

11c OMe

DAD1 B, Sig=230,4 Ref=off (02-Mar-2020102-Mar-2020 2020-03-03 10-23-141019-21-ml-eln-001-44.D)

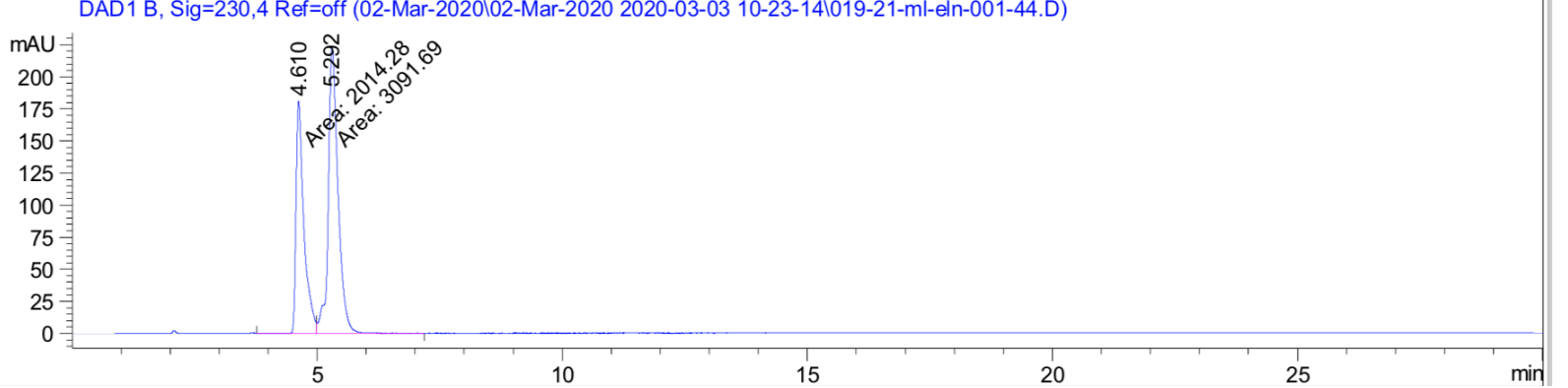

Signal 2: DAD1 B, Sig=230,4 Ref=off

\begin{tabular}{|c|c|c|c|c|c|c|}
\hline $\begin{array}{c}\text { Peak } \\
\quad \#\end{array}$ & $\begin{array}{c}\text { RetTime } \\
\text { [min] }\end{array}$ & Type & $\begin{array}{l}\text { Width } \\
\text { [min] }\end{array}$ & $\begin{array}{c}\text { Area } \\
{\left[\mathrm{mAU}^{*} \mathrm{~s}\right]}\end{array}$ & $\begin{array}{l}\text { Height } \\
\text { [mAU] }\end{array}$ & $\begin{array}{c}\text { Area } \\
\text { \% }\end{array}$ \\
\hline \multicolumn{7}{|c|}{$----|-------|----|-------|----------|----------|--------$} \\
\hline 1 & 4.610 & $\mathrm{MF}$ & 0.1855 & 2014.27637 & 180.93236 & 39.4495 \\
\hline 2 & 5.292 & FM & 0.2308 & 3091.69092 & 223.28300 & 60.5505 \\
\hline 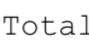 & S: & & & 5105.96729 & 404.21536 & \\
\hline
\end{tabular}


<smiles>COc1ccc(C(=N)c2ccc([N+](=O)[O-])cc2)cc1</smiles>

8d<smiles>C=Cc1ccccc1</smiles>

$\mathrm{Rh}_{2}(\mathrm{OAc})_{4} 1 \mathrm{~mol} \%$ $\mathrm{CH}_{2} \mathrm{Cl}_{2}, 23^{\circ} \mathrm{C}$

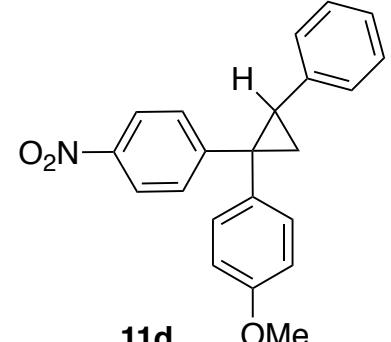

11d

DAD1 E, Sig=320,4 Ref=off (26-Jun-201...ation 2019-06-27 08-13-401005-10-ML-ELN-001-077Rh(OAc)pNO2pOMe.D)

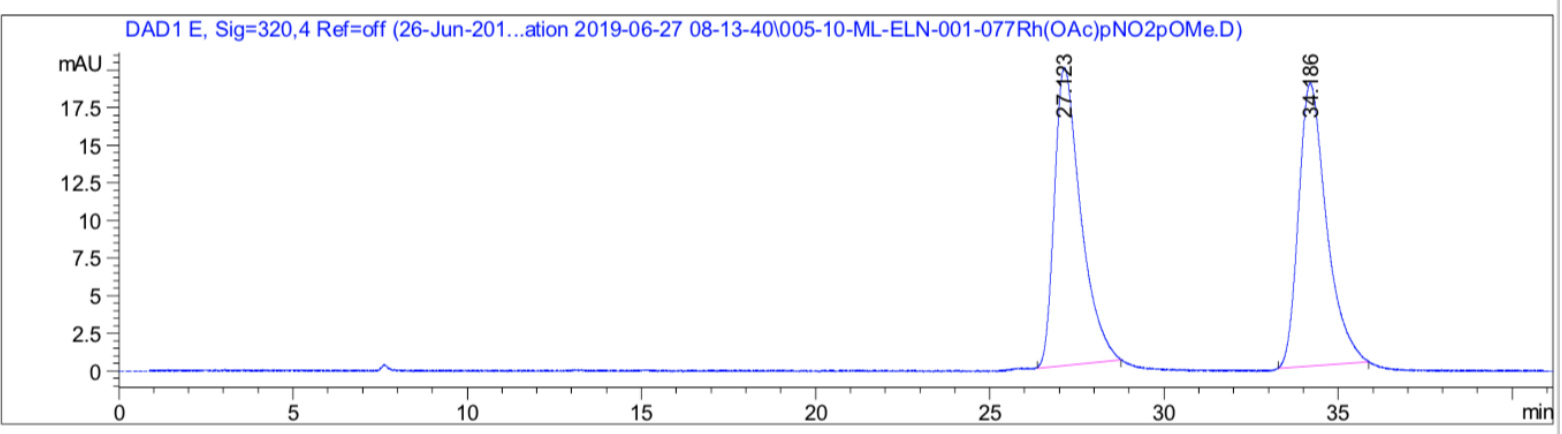

Signal 5: DAD1 E, Sig=320, 4 Ref=off

\begin{tabular}{|c|c|c|c|c|c|c|}
\hline $\begin{array}{c}\text { Peak } \\
\#\end{array}$ & $\begin{array}{c}\text { RetTime } \\
\text { [min] }\end{array}$ & Type & $\begin{array}{l}\text { Width } \\
\text { [min] }\end{array}$ & $\begin{array}{c}\text { Area } \\
{\left[\mathrm{mAU}{ }^{*} \mathrm{~s}\right]}\end{array}$ & $\begin{array}{l}\text { Height } \\
\text { [mAU] }\end{array}$ & $\begin{array}{c}\text { Area } \\
\%\end{array}$ \\
\hline & & & & | --------- & ----- & -----1 \\
\hline 1 & 27.123 & $B V R$ & 0.6166 & 1043.66797 & 19.81052 & 8348 \\
\hline 2 & 34.186 & $\mathrm{BB}$ & 0.6524 & 1050.58643 & 18.82965 & 50.1652 \\
\hline
\end{tabular}

Totals :

$2094.25439 \quad 38.64016$ 
<smiles>COc1ccc(C(=N)c2ccc([N+](=O)[O-])cc2)cc1</smiles>

8d

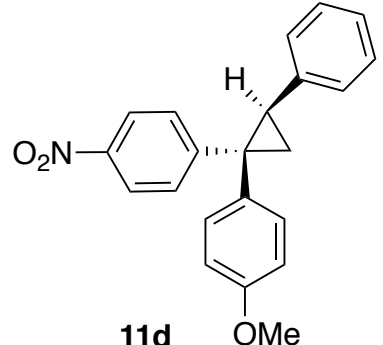

11d

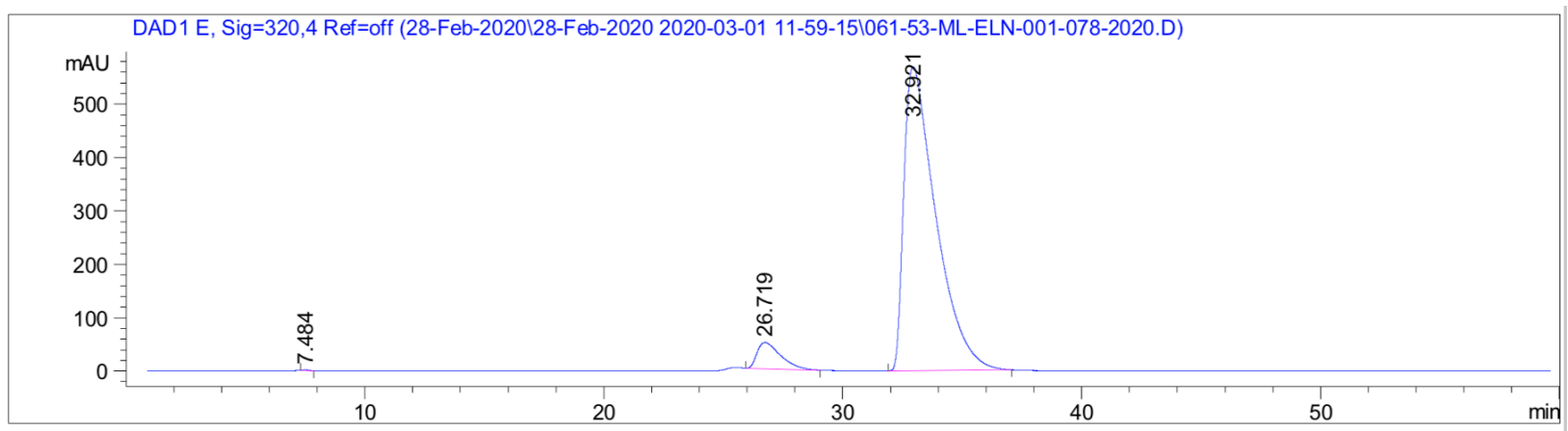

Signal 5: DAD1 E, Sig=320,4 Ref=off

\begin{tabular}{|c|c|c|c|c|c|c|}
\hline $\begin{array}{c}\text { eak } \\
\#\end{array}$ & $\begin{array}{c}\text { RetTime } \\
\text { [min] }\end{array}$ & Type & $\begin{array}{l}\text { Width } \\
\text { [min] }\end{array}$ & $\begin{array}{c}\text { Area } \\
{\left[m A{ }^{*} \mathrm{~s}\right]}\end{array}$ & $\begin{array}{l}\text { Height } \\
\text { [mAU] }\end{array}$ & $\begin{array}{c}\text { Area } \\
\frac{\circ}{0}\end{array}$ \\
\hline & & & & & --------- & --- \\
\hline 1 & & $B V \quad R$ & & 24.85926 & 1.76439 & 40 \\
\hline 2 & 26.7 & $\mathrm{VB} R$ & & 3372.18970 & 880 & 9751 \\
\hline 3 & 32.921 & VV $R$ & 883 & $5.30400 e 4$ & 569.88092 & 93.9808 \\
\hline
\end{tabular}

Totals :

5.64371 e $\quad 620.92411$ 
<smiles>COc1ccc(C(=N)c2ccc([N+](=O)[O-])cc2)cc1</smiles>

$8 d$<smiles>C=Cc1ccccc1</smiles>

$\mathrm{Rh}_{2}(\mathrm{~S}-\mathrm{NTTL})_{4} 1 \mathrm{~mol} \%$ $\mathrm{CH}_{2} \mathrm{Cl}_{2}, 23^{\circ} \mathrm{C}$

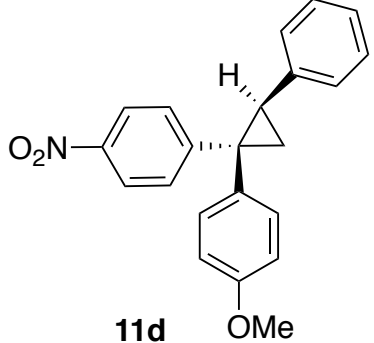

11d

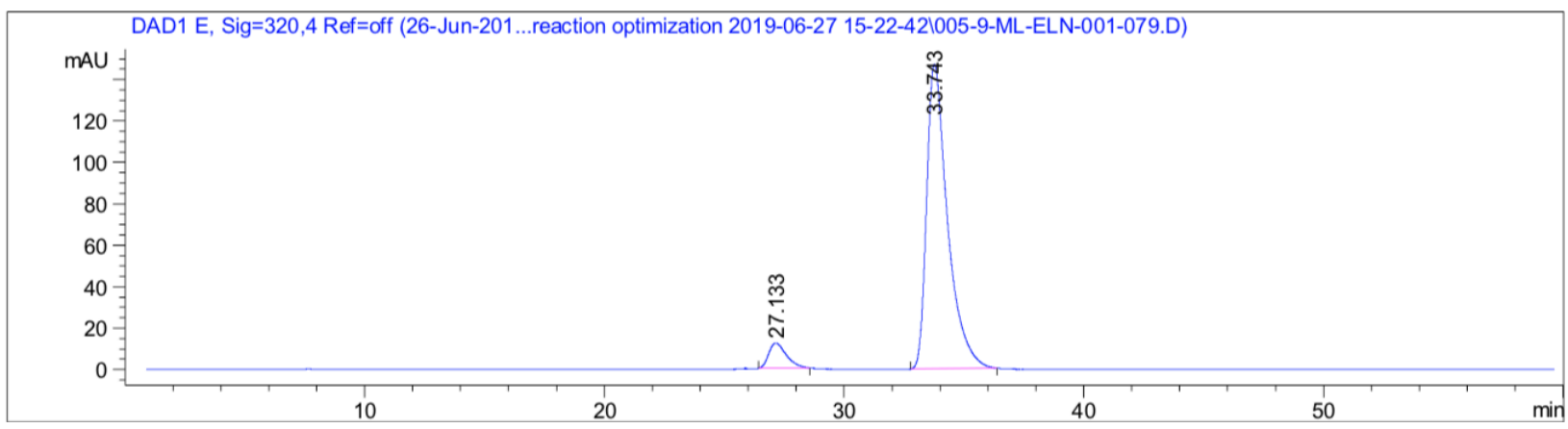

Signal 5: DAD1 E, Sig=320,4 Ref=off

\begin{tabular}{|c|c|c|c|c|c|c|}
\hline $\begin{array}{c}\text { Peak } \\
\#\end{array}$ & $\begin{array}{c}\text { RetTime } \\
\text { [min] }\end{array}$ & Type & $\begin{array}{l}\text { Width } \\
\text { [min] }\end{array}$ & $\begin{array}{c}\text { Area } \\
{\left[\mathrm{mAU}{ }^{\star} \mathrm{s}\right]}\end{array}$ & $\begin{array}{l}\text { Height } \\
\text { [mAU] }\end{array}$ & $\begin{array}{c}\text { Area } \\
\frac{\circ}{0}\end{array}$ \\
\hline & & & & --------- & 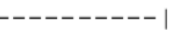 & \\
\hline 1 & & 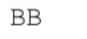 & & 627.57709 & 864 & 758 \\
\hline 2 & & & 09 & 8916.10840 & 5443 & 24 \\
\hline
\end{tabular}

Totals :

$9543.68549 \quad 159.25307$ 
<smiles>N#Cc1ccccc1Cl</smiles>

$+$<smiles>C=Cc1ccccc1</smiles>

$8 e$

\section{$\mathrm{Rh}_{2}(\mathrm{OAc})_{4} 1 \mathrm{~mol} \%$ $\mathrm{CH}_{2} \mathrm{Cl}_{2}, 23^{\circ} \mathrm{C}$}

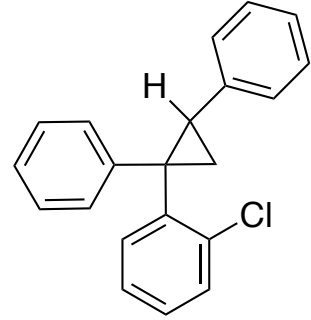

$11 \mathrm{e}$

DAD1 B, Sig=230,4 Ref=off (07-Sep-2019ISeptember-01-2019 2019-09-07 12-56-46l009-41-ML-ELN-001-165.D)

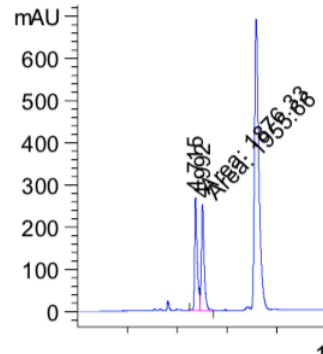

30

40

50

Signal 2: DAD1 B, Sig=230,4 Ref=off

\begin{tabular}{|c|c|c|c|c|c|c|}
\hline $\begin{array}{c}\text { Peak } \\
\#\end{array}$ & $\begin{array}{c}\text { RetTime } \\
\text { [min] }\end{array}$ & Type & $\begin{array}{l}\text { Width } \\
\text { [min] }\end{array}$ & $\begin{array}{c}\text { Area } \\
{\left[\mathrm{mAU}{ }^{\star} \mathrm{s}\right]}\end{array}$ & $\begin{array}{l}\text { Height } \\
\text { [mAU] }\end{array}$ & $\begin{array}{c}\text { Area } \\
\frac{\circ}{0}\end{array}$ \\
\hline & & & & ----1 & ----1 & $--------\mid$ \\
\hline 1 & & & & 1876.33179 & 267.18594 & 9649 \\
\hline 2 & 4.992 & FM & 0.1291 & 1955.66211 & 252.39343 & 0351 \\
\hline
\end{tabular}

Totals :

$3831.99390 \quad 519.57938$ 
<smiles>N#Cc1ccccc1C(=N)c1ccccc1</smiles>

$+$<smiles>C=Cc1ccccc1</smiles>

$\mathrm{Rh}_{2}(\mathrm{~S}-\mathrm{PTAD})_{4} 1 \mathrm{~mol} \%$ $\mathrm{CH}_{2} \mathrm{Cl}_{2}, 23^{\circ} \mathrm{C}$

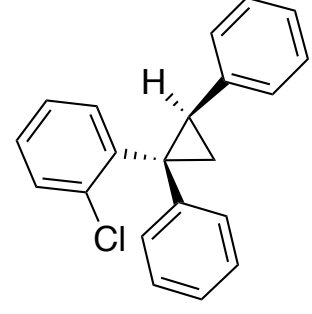

$11 e$

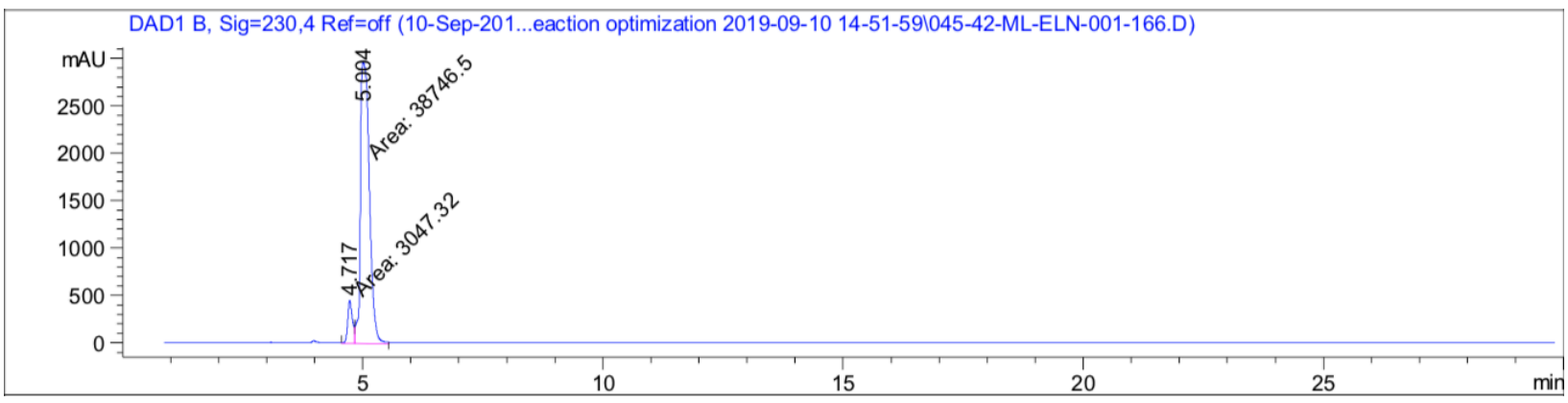

Signal 2: DAD1 B, Sig=230, 4 Ref=off

\begin{tabular}{|c|c|c|c|c|c|c|}
\hline $\begin{array}{c}\text { Peak } \\
\quad \#\end{array}$ & $\begin{array}{c}\text { RetTime } \\
\text { [min] }\end{array}$ & Type & $\begin{array}{l}\text { Width } \\
\text { [min] }\end{array}$ & $\begin{array}{c}\text { Area } \\
{\left[\mathrm{mAU}{ }^{*} \mathrm{~s}\right]}\end{array}$ & $\begin{array}{l}\text { Height } \\
\text { [mAU] }\end{array}$ & $\begin{array}{c}\text { Area } \\
\quad \frac{\circ}{0}\end{array}$ \\
\hline & ------ & & ------ & ----------- & | ---------- | & -------- \\
\hline 1 & 4.717 & $\mathrm{MF}$ & 0.1131 & 3047.31519 & 448.89621 & 7.2913 \\
\hline 2 & 5.004 & FM & 0.2170 & $3.87465 \mathrm{e} 4$ & 2975.54028 & 92.7087 \\
\hline otal & : & & & $4.17938 \mathrm{e} 4$ & 3424.43649 & \\
\hline
\end{tabular}


<smiles>N#Cc1ccccc1Cl</smiles>

$+$<smiles>C=Cc1ccccc1</smiles>

$\mathrm{Rh}_{2}(\mathrm{~S}-\mathrm{NTTL})_{4} 1 \mathrm{~mol} \%$ $\mathrm{CH}_{2} \mathrm{Cl}_{2}, 23^{\circ} \mathrm{C}$

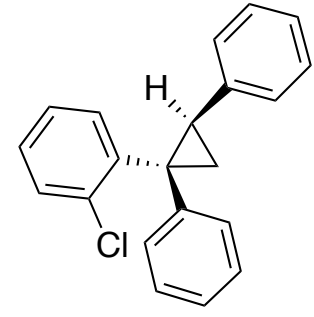

11e

$8 e$

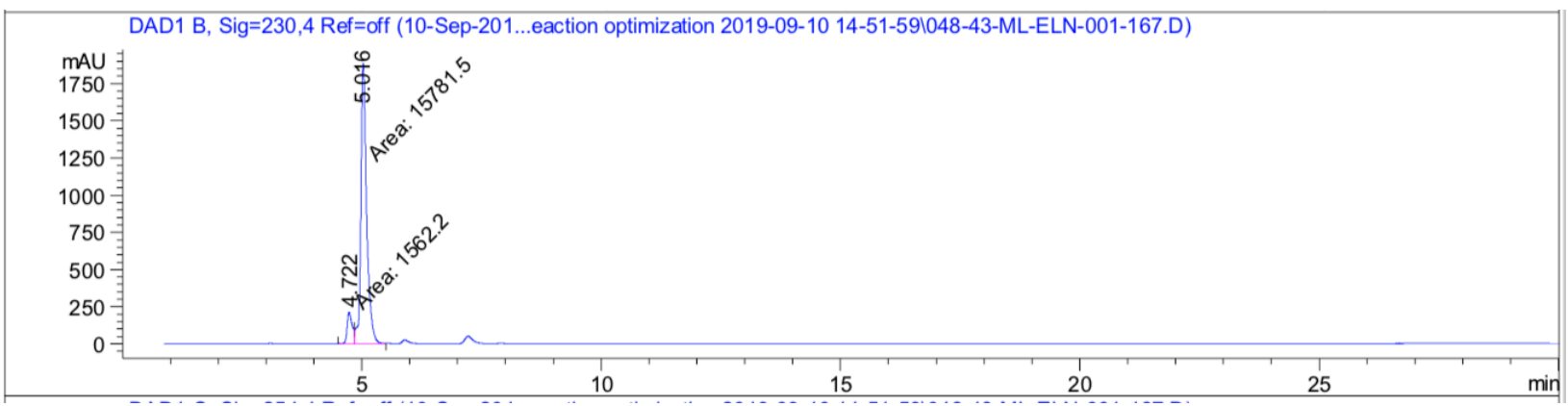

Signal 2: DAD1 B, Sig=230,4 Ref=off

\begin{tabular}{|c|c|c|c|c|c|c|}
\hline $\begin{array}{c}\text { Peak } \\
\quad \#\end{array}$ & $\begin{array}{c}\text { RetTime } \\
\text { [min] }\end{array}$ & Type & $\begin{array}{l}\text { Width } \\
\text { [min] }\end{array}$ & $\begin{array}{c}\text { Area } \\
{\left[\mathrm{mAU}{ }^{\star} \mathrm{S}\right]}\end{array}$ & $\begin{array}{l}\text { Height } \\
\text { [mAU] }\end{array}$ & $\begin{array}{c}\text { Area } \\
\frac{\circ}{0}\end{array}$ \\
\hline & -----1 & & & --------- & | ---------- & -------- \\
\hline 1 & 4.722 & $\mathrm{MF}$ & 0.1229 & 1562.20068 & 211.80057 & 9.0073 \\
\hline 2 & 5.016 & FM & 0.1395 & $1.57815 \mathrm{e} 4$ & 1885.17371 & 90.9927 \\
\hline tal & : & & & $1.73437 \mathrm{e} 4$ & 2096.97427 & \\
\hline
\end{tabular}



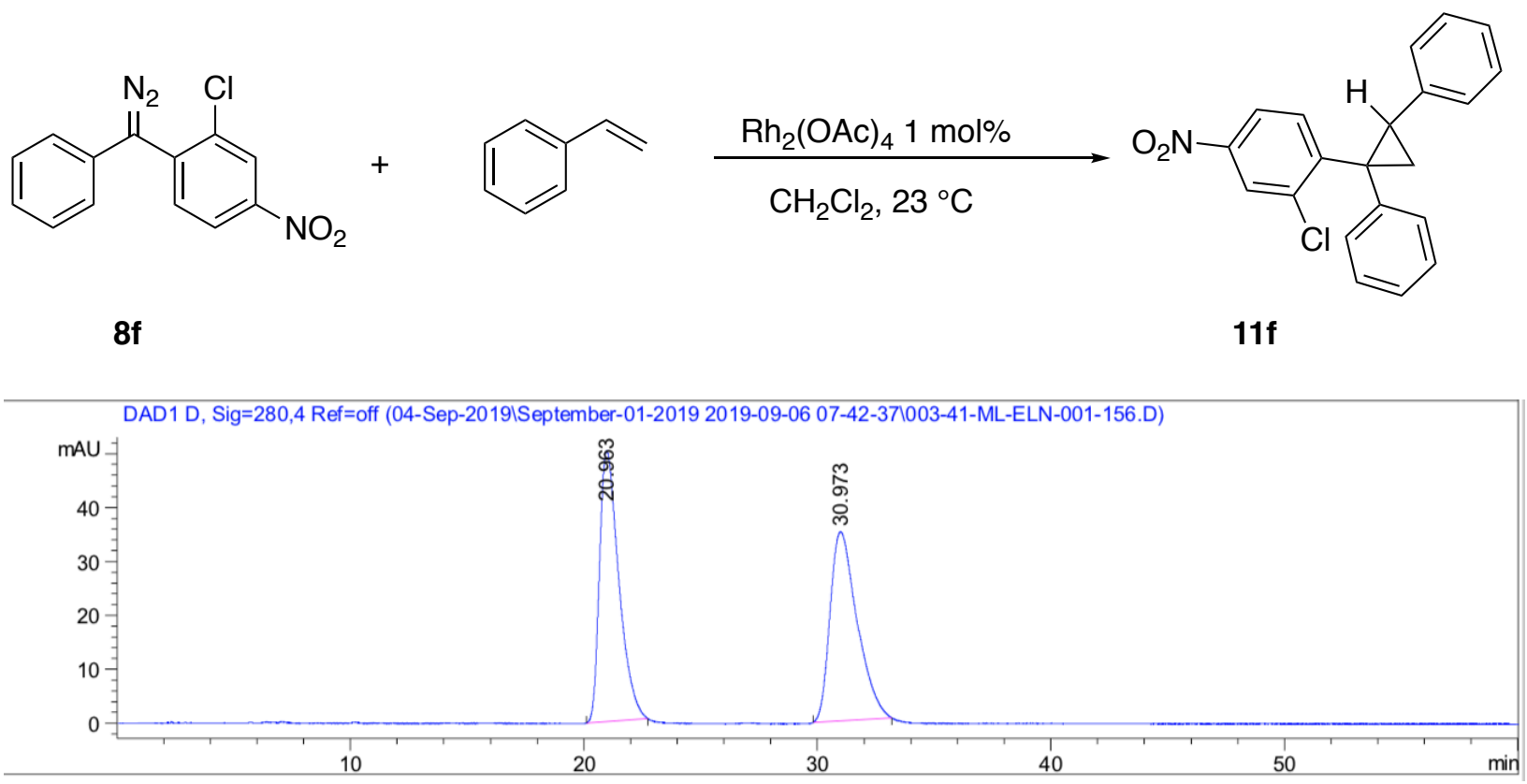

Signal 4: DAD1 D, Sig=280,4 Ref=off

\begin{tabular}{|c|c|c|c|c|c|c|}
\hline $\begin{array}{c}\text { Peak } \\
\#\end{array}$ & $\begin{array}{c}\text { RetTime } \\
\text { [min] }\end{array}$ & Type & $\begin{array}{l}\text { Width } \\
\text { [min] }\end{array}$ & $\begin{array}{c}\text { Area } \\
{\left[\mathrm{mAU}{ }^{*} \mathrm{~s}\right]}\end{array}$ & $\begin{array}{l}\text { Height } \\
\text { [mAU] }\end{array}$ & $\begin{array}{c}\text { Area } \\
\frac{\circ}{\delta}\end{array}$ \\
\hline - & ------ & & --- & |---ー-ー-ー- & -------- & -------1 \\
\hline 1 & 20.963 & BB & 0.6757 & 2891.30493 & 50.29194 & 50.6111 \\
\hline 2 & 30.973 & $\mathrm{BB}$ & 0.9418 & 2821.48755 & 35.06502 & 49.3889 \\
\hline
\end{tabular}

Totals : $\quad 5712.79248 \quad 85.35696$ 
<smiles>N#Cc1ccc([N+](=O)[O-])cc1Cl</smiles>

$8 f$<smiles>C=Cc1ccccc1</smiles>

$\mathrm{CH}_{2} \mathrm{Cl}_{2}, 23^{\circ} \mathrm{C}$

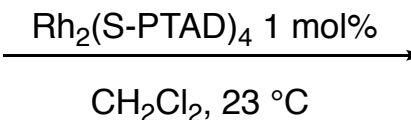

$\mathrm{O}_{2}$<smiles>OC1CCCCO1</smiles>

$11 f$

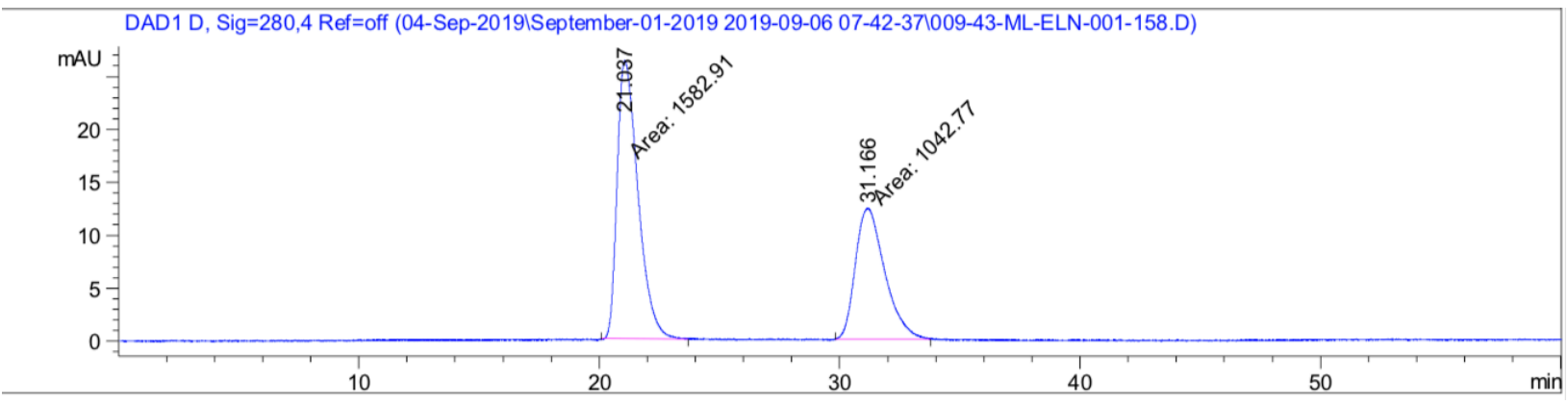

Signal 4: DAD1 D, Sig=280,4 Ref=off

\begin{tabular}{|c|c|c|c|c|c|c|}
\hline $\begin{array}{c}\text { Peak } \\
\quad \#\end{array}$ & $\begin{array}{l}\text { RetTime } \\
\text { [min] }\end{array}$ & Type & $\begin{array}{l}\text { Width } \\
\text { [min] }\end{array}$ & $\begin{array}{c}\text { Area } \\
{\left[\mathrm{mAU}{ }^{*} \mathrm{~s}\right]}\end{array}$ & $\begin{array}{l}\text { Height } \\
\text { [mAU] }\end{array}$ & $\begin{array}{c}\text { Area } \\
\frac{\circ}{\circ}\end{array}$ \\
\hline \multicolumn{7}{|c|}{$----|-------|----|-------|----------|----------|--------\mid$} \\
\hline 1 & 21.037 & MM & 1.0029 & 1582.91003 & 26.30664 & 60.2858 \\
\hline 2 & 31.166 & MM & 1.4096 & 1042.76563 & 12.32957 & 39.7142 \\
\hline a & : & & & 2625.67566 & 38.63621 & \\
\hline
\end{tabular}




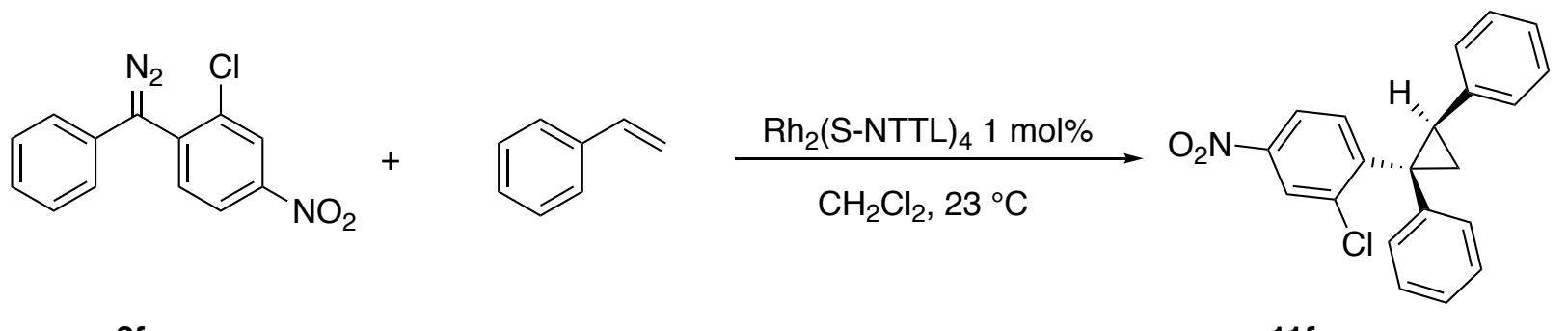

$8 f$

$11 f$

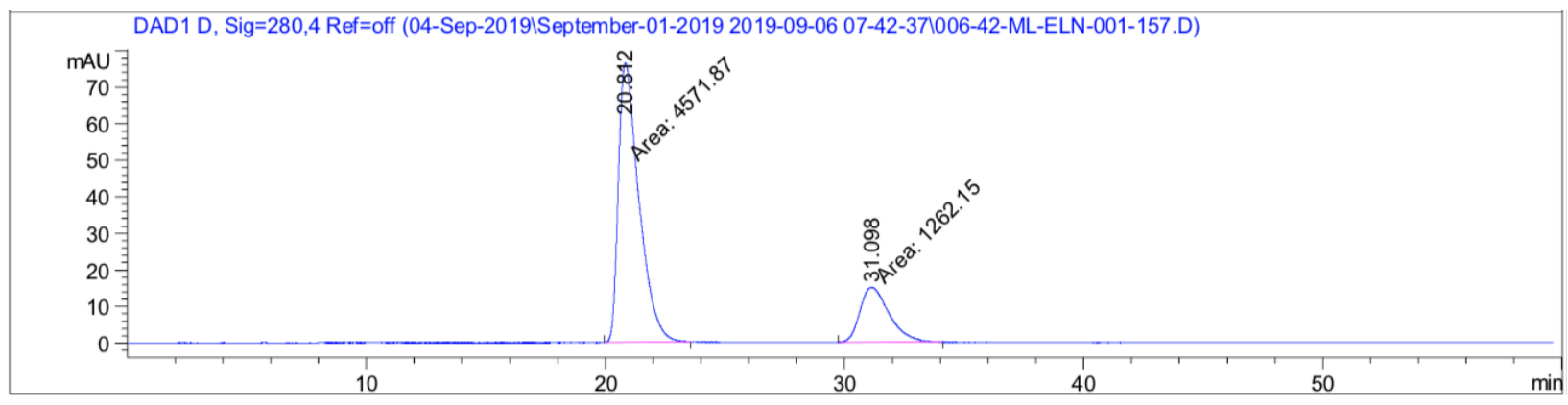

Signal 4: DAD1 D, Sig=280, 4 Ref=off

\begin{tabular}{|c|c|c|c|c|c|c|}
\hline $\begin{array}{c}\text { Peak } \\
\#\end{array}$ & $\begin{array}{c}\text { RetTime } \\
\text { [min] }\end{array}$ & Type & $\begin{array}{l}\text { Width } \\
\text { [min] }\end{array}$ & $\begin{array}{c}\text { Area } \\
{\left[\mathrm{mAU}{ }^{\star} \mathrm{s}\right]}\end{array}$ & $\begin{array}{l}\text { Height } \\
\text { [mAU] }\end{array}$ & $\begin{array}{c}\text { Area } \\
\frac{\circ}{0}\end{array}$ \\
\hline \multicolumn{7}{|c|}{$----|-------|----|-------|----------|----------|--------\mid$} \\
\hline 1 & 20.812 & MM & 0.9968 & 4571.86865 & 76.44129 & 78.3657 \\
\hline 2 & 31.098 & MM & 1.4080 & 1262.14636 & 14.94048 & 21.6343 \\
\hline Total & S : & & & 5834.01501 & 91.38177 & \\
\hline
\end{tabular}


<smiles>C=Cc1ccccc1</smiles>
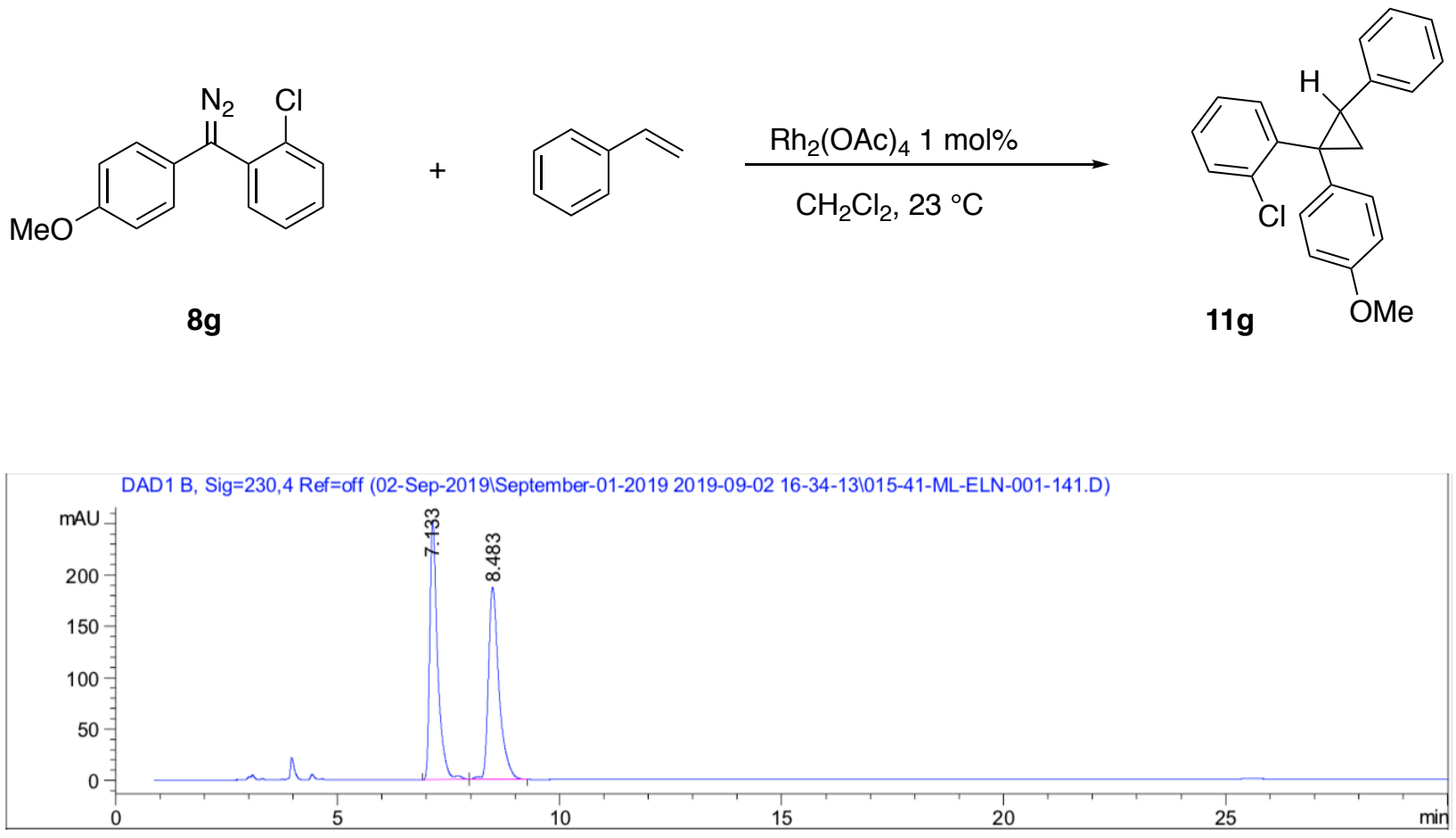

Signal 2: DAD1 B, Sig=230, 4 Ref=off

\begin{tabular}{|c|c|c|c|c|c|c|}
\hline $\begin{array}{c}\text { Peak } \\
\quad \#\end{array}$ & $\begin{array}{c}\text { RetTime } \\
\text { [min] }\end{array}$ & Type & $\begin{array}{l}\text { Width } \\
\text { [min] }\end{array}$ & $\begin{array}{c}\text { Area } \\
{\left[\mathrm{mAU}{ }^{\star} \mathrm{s}\right]}\end{array}$ & $\begin{array}{l}\text { Height } \\
\text { [mAU] }\end{array}$ & $\begin{array}{c}\text { Area } \\
\quad \%\end{array}$ \\
\hline \multicolumn{7}{|c|}{$----|-------|----|-------|----------|----------|--------\mid$} \\
\hline 1 & 7.133 & BV R & 0.1797 & 3100.63843 & 252.68707 & 50.0674 \\
\hline 2 & 8.483 & VB $R$ & 0.2428 & 3092.28613 & 186.84401 & 49.9326 \\
\hline Total & ls : & & & 6192.92456 & 439.53108 & \\
\hline
\end{tabular}


到

$8 g$

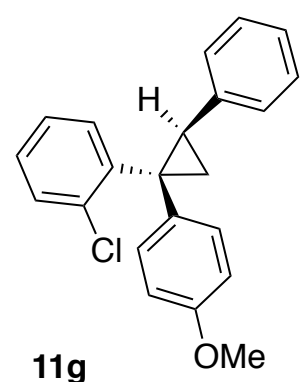

$11 g$

DAD1 B, Sig=230,4 Ref=off (10-Sep-201...eaction optimization 2019-09-10 14-51-59l051-11-ML-ELN-001-140.D)

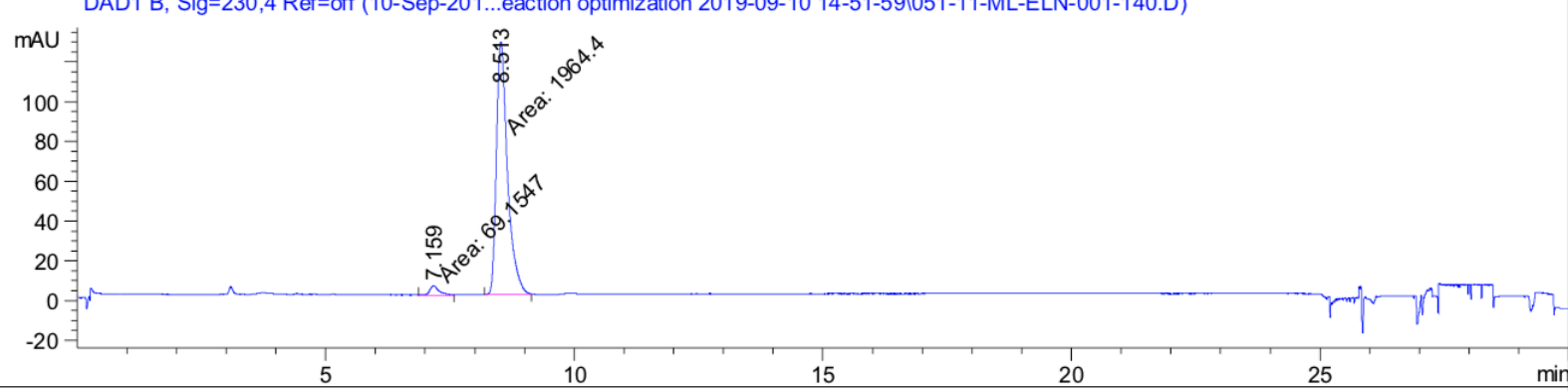

Signal 2: DAD1 B, Sig=230, 4 Ref=off

\begin{tabular}{|c|c|c|c|c|c|c|}
\hline $\begin{array}{c}\text { Peak } \\
\#\end{array}$ & $\begin{array}{c}\text { RetTime } \\
\text { [min] }\end{array}$ & Type & $\begin{array}{l}\text { Width } \\
\text { [min] }\end{array}$ & $\begin{array}{c}\text { Area } \\
{\left[\mathrm{mAU}{ }^{*} \mathrm{~s}\right]}\end{array}$ & $\begin{array}{l}\text { Height } \\
\text { [mAU] }\end{array}$ & $\begin{array}{c}\text { Area } \\
\frac{\circ}{0}\end{array}$ \\
\hline \multicolumn{7}{|c|}{$----|-------|----|-------|----------|----------|--------\mid$} \\
\hline 1 & 7.159 & MM & 0.2355 & 69.15466 & 4.89337 & 3.4007 \\
\hline 2 & 8.513 & MM & 0.2581 & 1964.40234 & 126.85229 & 96.5993 \\
\hline Total & : & & & 2033.55701 & 131.74566 & \\
\hline
\end{tabular}


<smiles>C=Cc1ccccc1</smiles>

$8 g$
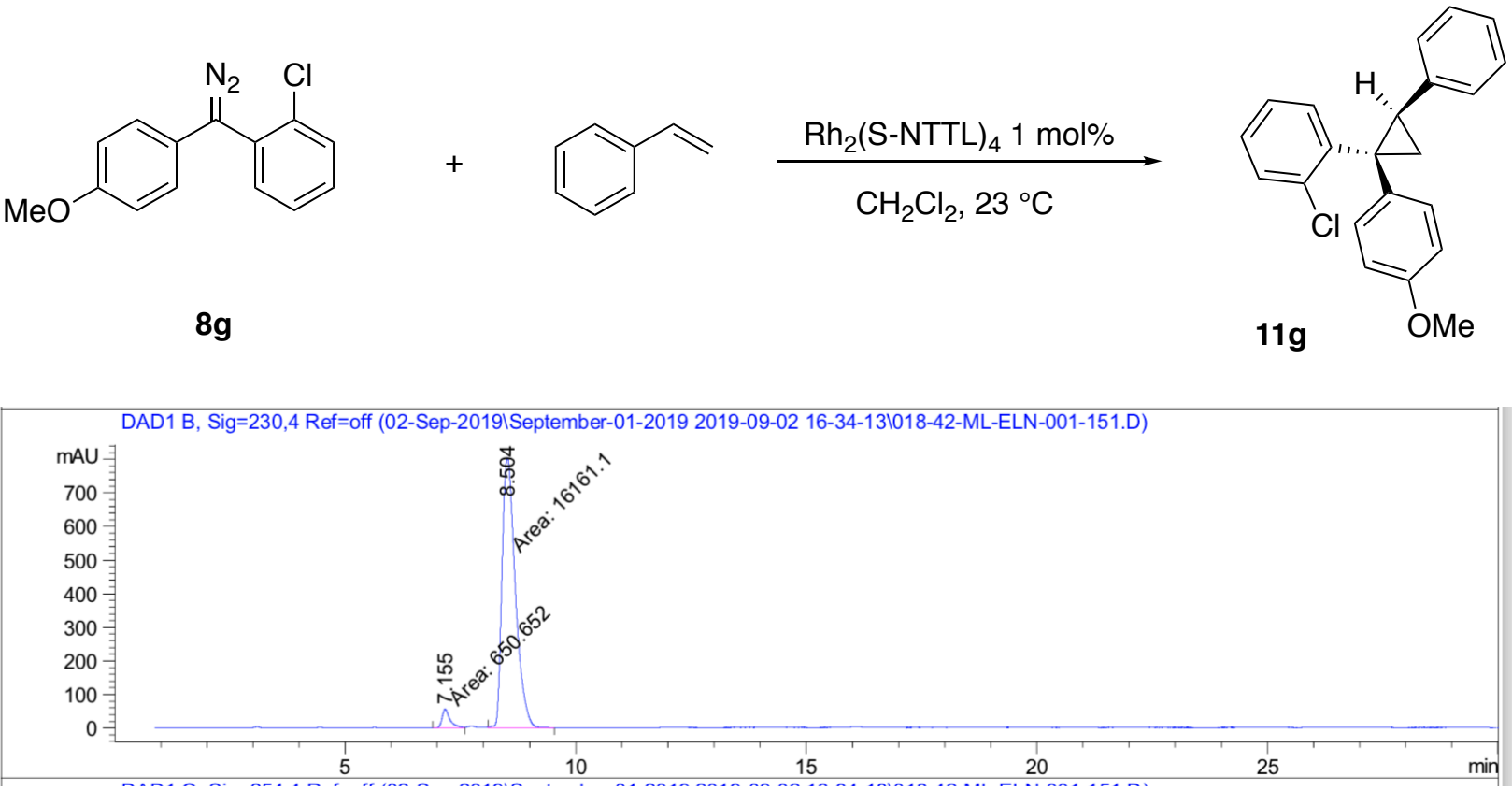

Signal 2: DAD1 B, Sig=230,4 Ref=off

\begin{tabular}{|c|c|c|c|c|c|c|}
\hline $\begin{array}{c}\text { Peak } \\
\#\end{array}$ & $\begin{array}{c}\text { RetTime } \\
\text { [min] }\end{array}$ & Type & $\begin{array}{l}\text { Width } \\
\text { [min] }\end{array}$ & $\begin{array}{c}\text { Area } \\
{\left[\mathrm{mAU}{ }^{\star} \mathrm{s}\right]}\end{array}$ & $\begin{array}{l}\text { Height } \\
\text { [mAU] }\end{array}$ & $\begin{array}{c}\text { Area } \\
\frac{\circ}{0}\end{array}$ \\
\hline & & & & --ー-ー-ー--- & ----------1 & --------1 \\
\hline 1 & 7.155 & MM & 0.1949 & 645.79358 & 55.21113 & 3.9143 \\
\hline 2 & 8.504 & MM & 0.3302 & $1.58525 \mathrm{e} 4$ & 800.06903 & 96.0857 \\
\hline
\end{tabular}

Totals :

$1.64983 \mathrm{e} 4 \quad 855.28016$ 


\section{Computational Calculations}

Calculation methods: Gaussian09 program at E01 version ${ }^{13}$ was used for the calculations, B3LYP level theory ${ }^{14}$ with D3BJ function for dispersion correction ${ }^{15}$ was used, along with the basis set 6$31 \mathrm{G}(\mathrm{d}, \mathrm{p})$ for main group elements and LANL2DZ for $\mathrm{Rh}$ atom. ${ }^{16}$ The solvent effect was incorporated by employing the PCM model with dichloromethane as solvent. ${ }^{17}$ The images were generated by Mercury for the structures and the molecular orbitals were generated by GaussView. The image of the lowest unoccupied molecular orbital was obtained from the formatted checkpoint file with GaussView. The intermediate such as dirhodium carbenes are calculated, and there is no imaginary frequency for the optimized structures. The TS structures obtained have one imaginary frequency and the forward and reverse IRC calculations for representative TS were conducted for 50 steps to connect to the starting material and product of the TS.

\section{Rh carbene structures:}

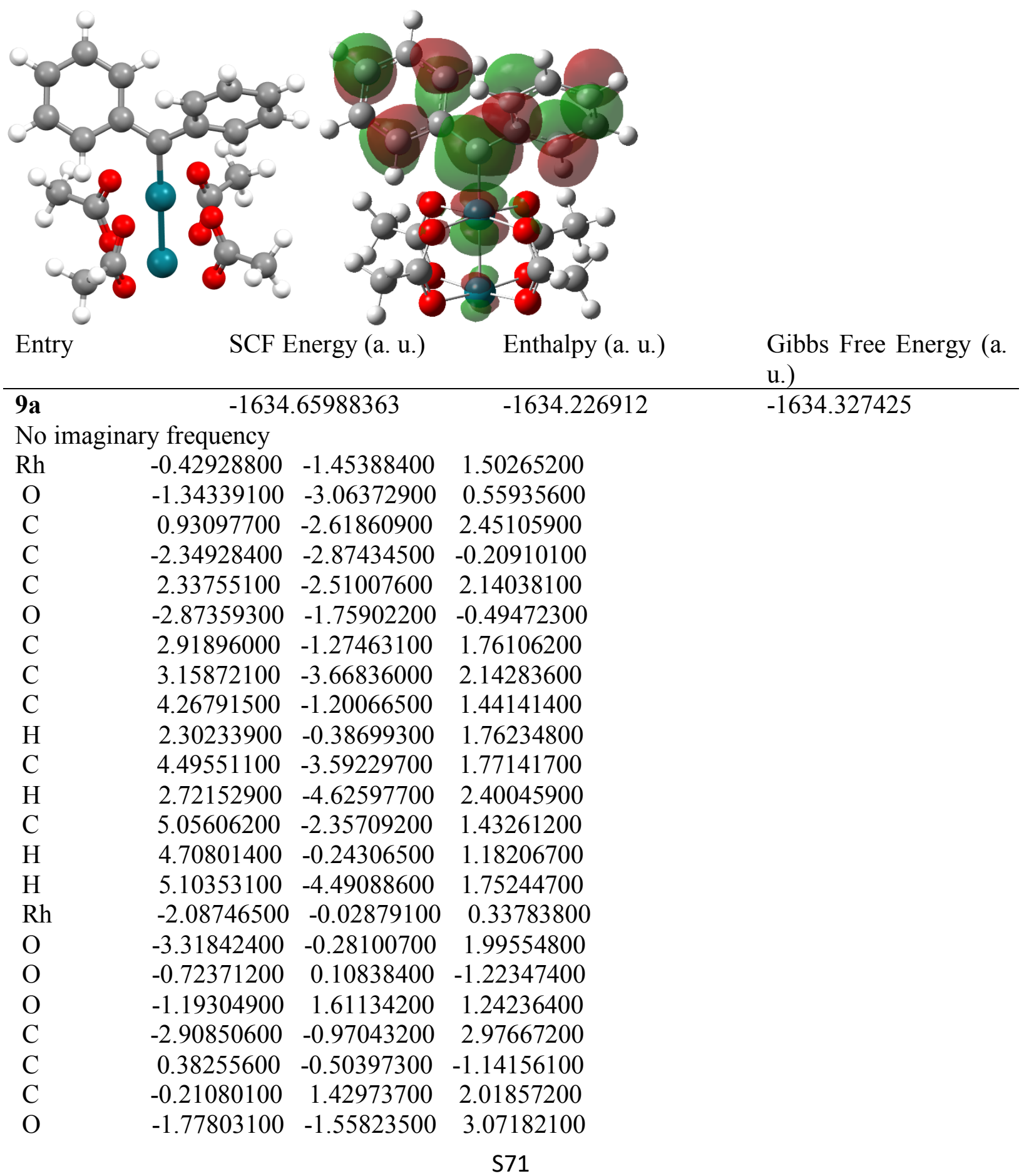




$\begin{array}{lrrr}\mathrm{O} & 0.77731500 & -1.22967500 & -0.16688000 \\ \mathrm{O} & 0.31040400 & 0.30279400 & 2.32994300 \\ \mathrm{C} & 1.33642500 & -0.39942700 & -2.30830500 \\ \mathrm{H} & 2.35743400 & -0.27066400 & -1.94302000 \\ \mathrm{H} & 1.30129900 & -1.33232900 & -2.88017100 \\ \mathrm{H} & 1.05691400 & 0.42816900 & -2.96024600 \\ \mathrm{C} & -2.95363100 & -4.12002800 & -0.81195400 \\ \mathrm{H} & -3.36553700 & -4.74242200 & -0.01190000 \\ \mathrm{H} & -3.74259700 & -3.86188100 & -1.51776400 \\ \mathrm{H} & -2.17545600 & -4.70032400 & -1.31412000 \\ \mathrm{C} & -3.81901500 & -1.10078000 & 4.17510900 \\ \mathrm{H} & -3.72314900 & -2.09612300 & 4.61264900 \\ \mathrm{H} & -3.51468900 & -0.36887500 & 4.93066500 \\ \mathrm{H} & -4.85348700 & -0.90345000 & 3.89291000 \\ \mathrm{C} & 0.43751500 & 2.64341200 & 2.64055300 \\ \mathrm{H} & 0.55101200 & 2.49218000 & 3.71659200 \\ \mathrm{H} & 1.43844600 & 2.76983000 & 2.21632800 \\ \mathrm{H} & -0.15427400 & 3.53747400 & 2.44592200 \\ \mathrm{C} & 0.51738200 & -3.57470400 & 3.45165100 \\ \mathrm{C} & -0.73164200 & -4.23917500 & 3.36919100 \\ \mathrm{C} & 1.33593500 & -3.81655500 & 4.58632900 \\ \mathrm{C} & -1.11889900 & -5.13331400 & 4.35809300 \\ \mathrm{H} & -1.35838100 & -4.06536200 & 2.50585900 \\ \mathrm{C} & 0.91591400 & -4.67461600 & 5.59531100 \\ \mathrm{H} & 2.27974500 & -3.29191500 & 4.67837900 \\ \mathrm{C} & -0.30569600 & -5.34429400 & 5.47737700 \\ \mathrm{H} & -2.06427600 & -5.65885900 & 4.26874900 \\ \mathrm{H} & 1.54047100 & -4.82873400 & 6.46921000 \\ \mathrm{H} & 6.10430700 & -2.29602400 & 1.15677300 \\ \mathrm{H} & -0.62650200 & -6.02593500 & 6.25910700\end{array}$

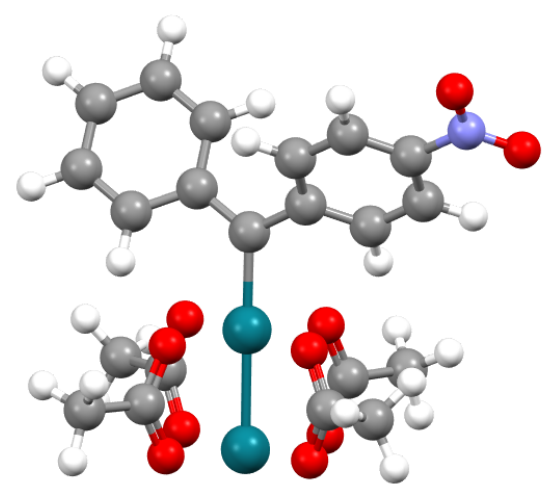

$\begin{array}{lll}\text { Entry } & \text { SCF Energy (a. u.) } \quad \text { Enthalpy (a. u.) } \quad \text { Gibbs Free Energy (a. }\end{array}$ u.)

\begin{tabular}{lrcc}
\hline 9b & \multicolumn{1}{c}{-1839.16219591} & -1838.72 \\
No imaginary frequency & & \\
Rh & -0.49721200 & -1.39323000 & 1.45517500 \\
O & -1.47491700 & -2.93801200 & 0.46521000 \\
C & 0.76581300 & -2.64971200 & 2.41085400 \\
C & -2.46018800 & -2.68817400 & -0.31317600 \\
C & 2.18013600 & -2.65911300 & 2.16282800 \\
O & -2.92156900 & -1.54317300 & -0.58746100 \\
C & 2.85694700 & -1.48873900 & 1.72897300 \\
C & 2.92718800 & -3.86188500 & 2.30005300
\end{tabular}




$\begin{array}{lrrr}\mathrm{C} & 4.22089700 & -1.52054400 & 1.47714800 \\ \mathrm{H} & 2.29945600 & -0.56754700 & 1.63950400 \\ \mathrm{C} & 4.28072600 & -3.89300400 & 1.99725900 \\ \mathrm{H} & 2.41941300 & -4.76875000 & 2.60691100 \\ \mathrm{C} & 4.93143400 & -2.72062900 & 1.59633600 \\ \mathrm{H} & 4.73527900 & -0.61449500 & 1.17430300 \\ \mathrm{H} & 4.83368900 & -4.82280200 & 2.07870100 \\ \mathrm{H} & 5.99380000 & -2.74323700 & 1.37411900 \\ \mathrm{Rh} & -2.05875900 & 0.12654300 & 0.28956700 \\ \mathrm{O} & -3.33304200 & -0.08996300 & 1.91708900 \\ \mathrm{O} & -0.66528300 & 0.22261100 & -1.24698600 \\ \mathrm{O} & -1.09738200 & 1.70003700 & 1.24146500 \\ \mathrm{C} & -2.97632200 & -0.81397600 & 2.89380500 \\ \mathrm{C} & 0.40366500 & -0.45218800 & -1.16274100 \\ \mathrm{C} & -0.12593300 & 1.45948400 & 2.01485800 \\ \mathrm{O} & -1.87560800 & -1.45604300 & 2.99834800 \\ \mathrm{O} & 0.74457300 & -1.21254500 & -0.19338100 \\ \mathrm{O} & 0.33651300 & 0.30223100 & 2.30909600 \\ \mathrm{C} & 1.37317800 & -0.38817800 & -2.31866700 \\ \mathrm{H} & 2.39832300 & -0.35509100 & -1.94458000 \\ \mathrm{H} & 1.26497900 & -1.29603900 & -2.92101900 \\ \mathrm{H} & 1.16595500 & 0.47939800 & -2.94512500 \\ \mathrm{C} & -3.11998500 & -3.89190700 & -0.94057800 \\ \mathrm{H} & -3.58864700 & -4.49289300 & -0.15541800 \\ \mathrm{H} & -3.87459400 & -3.58307100 & -1.66337500 \\ \mathrm{H} & -2.36491000 & -4.51422600 & -1.42708600 \\ \mathrm{C} & -3.91510900 & -0.93041900 & 4.07072900 \\ \mathrm{H} & -3.97736600 & -1.97207400 & 4.39318200 \\ \mathrm{H} & -3.51249100 & -0.34713900 & 4.90497100 \\ \mathrm{H} & -4.90393000 & -0.55153600 & 3.81302700 \\ \mathrm{C} & 0.58291700 & 2.62863200 & 2.65431900 \\ \mathrm{H} & 0.68624000 & 2.45697000 & 3.72828200 \\ \mathrm{H} & 1.58979100 & 2.70822300 & 2.23284800 \\ \mathrm{H} & 0.03870700 & 3.55461800 & 2.47077300 \\ \mathrm{C} & 0.22648300 & -3.56935000 & 3.39897600 \\ \mathrm{C} & -1.00627500 & -4.23250800 & 3.19966100 \\ \mathrm{C} & 0.90248300 & -3.73949300 & 4.63178500 \\ \mathrm{C} & -1.52587800 & -5.06395800 & 4.17938600 \\ \mathrm{H} & -1.52823800 & -4.09816400 & 2.26238500 \\ \mathrm{C} & 0.36423800 & -4.53379500 & 5.63490300 \\ \mathrm{H} & 1.83665500 & -3.21888200 & 4.80448700 \\ \mathrm{H} & -0.83823700 & -5.19324000 & 5.38704700 \\ \mathrm{H} & -2.45696900 & -5.59497700 & 4.03229400 \\ 0.86310300 & -4.65814300 & 6.58679300 \\ \mathrm{H} & -1.40246100 & -6.04917100 & 6.43401300 \\ \mathrm{H} & -2.46111800 & -6.63267700 & 6.19470600\end{array}$




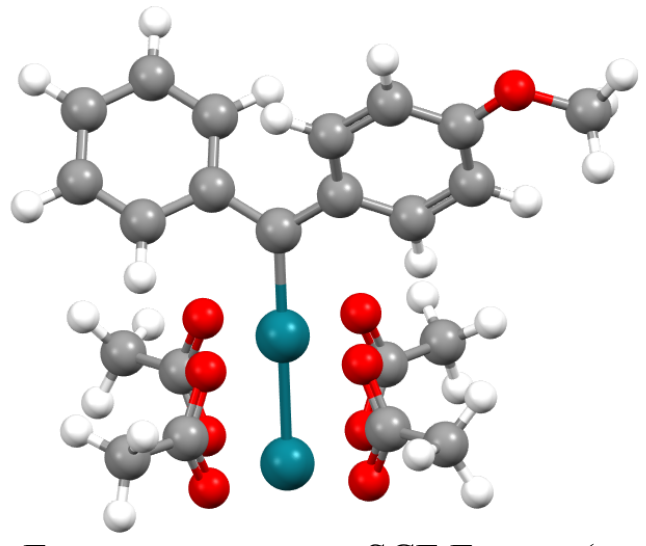

Entry SCF Energy (a. u

Enthalpy (a. u.)

Gibbs Free Energy (a. u.)

\begin{tabular}{lrcc}
\hline 9c & -1748.729239 & -1748.72 \\
No imaginary frequency & & \\
Rh & -0.43753200 & -1.44416000 & 1.52047000 \\
$\mathrm{O}$ & -1.33307000 & -3.08428700 & 0.61368800 \\
$\mathrm{C}$ & 0.91227600 & -2.60225700 & 2.51168000 \\
$\mathrm{C}$ & -2.31886700 & -2.91663900 & -0.18535600 \\
$\mathrm{C}$ & 2.32239500 & -2.47267900 & 2.19084700 \\
$\mathrm{O}$ & -2.83364400 & -1.81029500 & -0.51966000 \\
$\mathrm{C}$ & 2.90851500 & -1.21536800 & 1.91550600 \\
$\mathrm{C}$ & 3.12802700 & -3.63175400 & 2.06503600 \\
$\mathrm{C}$ & 4.25251900 & -1.12331500 & 1.57599200 \\
$\mathrm{H}$ & 2.29747900 & -0.32702100 & 1.99919600 \\
$\mathrm{C}$ & 4.46009200 & -3.53382600 & 1.67593900 \\
$\mathrm{H}$ & 2.68474700 & -4.60586500 & 2.23845700 \\
$\mathrm{C}$ & 5.02981800 & -2.27932400 & 1.44380000 \\
$\mathrm{H}$ & 4.69550400 & -0.14887300 & 1.39503800 \\
$\mathrm{H}$ & 5.05587000 & -4.43368300 & 1.56033500 \\
$\mathrm{Rh}$ & -2.07345000 & -0.05165700 & 0.28281600 \\
$\mathrm{O}$ & -3.34029100 & -0.26127000 & 1.92157600 \\
$\mathrm{O}$ & -0.67455800 & 0.04821100 & -1.25066700 \\
$\mathrm{O}$ & -1.20286900 & 1.61377900 & 1.16423900 \\
$\mathrm{C}$ & -2.94559200 & -0.91435700 & 2.93320600 \\
$\mathrm{C}$ & 0.42919200 & -0.56314900 & -1.13001700 \\
$\mathrm{C}$ & -0.23893100 & 1.45438100 & 1.96845200 \\
$\mathrm{O}$ & -1.81607100 & -1.49626000 & 3.06870600 \\
$\mathrm{O}$ & 0.80376200 & -1.26375900 & -0.13013000 \\
$\mathrm{O}$ & 0.27701200 & 0.33820900 & 2.32151200 \\
$\mathrm{C}$ & 1.40518000 & -0.48946200 & -2.28127800 \\
$\mathrm{H}$ & 2.42252300 & -0.37892400 & -1.90045900 \\
$\mathrm{H}$ & 1.35875900 & -1.42637000 & -2.84593900 \\
$\mathrm{H}$ & 1.15370600 & 0.33729200 & -2.94574800 \\
$\mathrm{C}$ & -2.90991500 & -4.17986300 & -0.76558000 \\
$\mathrm{H}$ & -3.31337000 & -4.79391400 & 0.04498500 \\
$\mathrm{H}$ & -3.70146300 & -3.94351900 & -1.47611900 \\
$\mathrm{H}$ & -2.12501400 & -4.75922400 & -1.25871200 \\
$\mathrm{C}$ & -3.87608300 & -1.00312200 & 4.12057400 \\
$\mathrm{H}$ & -3.79524300 & -1.98602700 & 4.58844900 \\
$\mathrm{H}$ & -3.57745700 & -0.25238100 & 4.85977000 \\
$\mathrm{H}$ & -4.90457800 & -0.80639300 & 3.81696800 \\
$\mathrm{C}$ & 0.39131200 & 2.68644900 & 2.57359400
\end{tabular}




$\begin{array}{lrrr}\mathrm{H} & 0.48158000 & 2.56316500 & 3.65539900 \\ \mathrm{H} & 1.40107200 & 2.80570900 & 2.16855200 \\ \mathrm{H} & -0.19851300 & 3.57335300 & 2.34331400 \\ \mathrm{C} & 0.51475600 & -3.56704200 & 3.48782600 \\ \mathrm{C} & -0.78273200 & -4.14738100 & 3.47765400 \\ \mathrm{C} & 1.39400900 & -3.95204300 & 4.54607100 \\ \mathrm{C} & -1.16852500 & -5.07920800 & 4.42413000 \\ \mathrm{H} & -1.46151600 & -3.88603200 & 2.67927500 \\ \mathrm{C} & 0.99964000 & -4.84170900 & 5.51996500 \\ \mathrm{H} & 2.37697100 & -3.50043200 & 4.60493200 \\ \mathrm{C} & -0.28283300 & -5.42650500 & 5.46237700 \\ \mathrm{H} & -2.15214500 & -5.52644100 & 4.36205100 \\ \mathrm{H} & 1.65522100 & -5.11070700 & 6.34071800 \\ \mathrm{H} & 6.07303800 & -2.20237000 & 1.15321100 \\ \mathrm{O} & -0.56863200 & -6.29770700 & 6.44440600 \\ \mathrm{C} & -1.85640500 & -6.92422000 & 6.46079200 \\ \mathrm{H} & -1.85553800 & -7.57526800 & 7.33384400 \\ \mathrm{H} & -2.65248800 & -6.17942600 & 6.55610100 \\ \mathrm{H} & -2.01295800 & -7.52024000 & 5.55640800\end{array}$

\begin{tabular}{|c|c|c|c|}
\hline Entry & SCF I & inergy (a. u.) & Enthalpy \\
\hline 9d & -1953 & 70153547 & -1953.22 \\
\hline \multicolumn{4}{|c|}{ No imaginary frequency } \\
\hline $\mathrm{Rh}$ & -0.49323200 & -1.36918400 & 1.46254300 \\
\hline $\mathrm{O}$ & -1.49732500 & -2.92321000 & 0.50924000 \\
\hline $\mathrm{C}$ & 0.82183800 & -2.61210900 & 2.39295000 \\
\hline $\mathrm{C}$ & -2.51055800 & -2.67950400 & -0.23266200 \\
\hline $\mathrm{C}$ & 2.21992500 & -2.60237100 & 2.14931100 \\
\hline $\mathrm{O}$ & -2.99093400 & -1.53771700 & -0.49015800 \\
\hline $\mathrm{C}$ & 2.87490900 & -1.46654200 & 1.57982400 \\
\hline $\mathrm{C}$ & 3.02054700 & -3.75176700 & 2.42143200 \\
\hline $\mathrm{C}$ & 4.22598600 & -1.48012800 & 1.32893000 \\
\hline $\mathrm{H}$ & 2.29616100 & -0.57526900 & 1.38878200 \\
\hline $\mathrm{C}$ & 4.36872500 & -3.78885600 & 2.12757500 \\
\hline $\mathrm{H}$ & 2.54810500 & -4.63649700 & 2.83147200 \\
\hline $\mathrm{C}$ & 4.98669300 & -2.64348100 & 1.58383600 \\
\hline $\mathrm{H}$ & 4.73514300 & -0.61232600 & 0.92475000 \\
\hline $\mathrm{H}$ & 4.93570800 & -4.69080600 & 2.31668600 \\
\hline $\mathrm{Rh}$ & -2.10811700 & 0.14054700 & 0.35129000 \\
\hline $\mathrm{O}$ & -3.31509600 & -0.07710300 & 2.02942300 \\
\hline
\end{tabular}




$\begin{array}{lrrr}\mathrm{O} & -0.77541600 & 0.23821800 & -1.23852100 \\ \mathrm{O} & -1.11513500 & 1.71871100 & 1.26732300 \\ \mathrm{C} & -2.91690500 & -0.79529700 & 2.99473500 \\ \mathrm{C} & 0.29465100 & -0.43867800 & -1.19748800 \\ \mathrm{C} & -0.11368400 & 1.48315100 & 2.00364000 \\ \mathrm{O} & -1.81189000 & -1.43377800 & 3.05910400 \\ \mathrm{O} & 0.67653900 & -1.19440800 & -0.24027400 \\ \mathrm{O} & 0.37183400 & 0.33017600 & 2.27516700 \\ \mathrm{C} & 1.21112100 & -0.38347800 & -2.39677200 \\ \mathrm{H} & 2.25274600 & -0.35658800 & -2.07088100 \\ \mathrm{H} & 1.06896100 & -1.29166100 & -2.99164600 \\ \mathrm{H} & 0.98121000 & 0.48399400 & -3.01549400 \\ \mathrm{C} & -3.18467600 & -3.88736000 & -0.83808700 \\ \mathrm{H} & -3.62520600 & -4.48980700 & -0.03781500 \\ \mathrm{H} & -3.96374200 & -3.58351000 & -1.53668500 \\ \mathrm{H} & -2.44266300 & -4.50685300 & -1.34778300 \\ \mathrm{C} & -3.80911400 & -0.90719400 & 4.20843900 \\ \mathrm{H} & -3.86087400 & -1.94797800 & 4.53568800 \\ \mathrm{H} & -3.37304700 & -0.32300000 & 5.02505200 \\ \mathrm{H} & -4.80678700 & -0.52720600 & 3.98918000 \\ \mathrm{C} & 0.59162400 & 2.65539700 & 2.64278400 \\ \mathrm{H} & 0.50469900 & 2.57974600 & 3.73057800 \\ \mathrm{H} & 1.65551700 & 2.62170300 & 2.39374900 \\ \mathrm{H} & 0.15819300 & 3.59610300 & 2.30456900 \\ \mathrm{C} & 0.28502100 & -3.52599100 & 3.39394600 \\ \mathrm{C} & -0.87763700 & -4.29211300 & 3.15709700 \\ \mathrm{C} & 0.88685600 & -3.57646300 & 4.67321700 \\ \mathrm{C} & -1.39935100 & -5.10718800 & 4.14930700 \\ \mathrm{H} & -1.35255900 & -4.23736500 & 2.18682900 \\ \mathrm{C} & 0.34605300 & -4.35903000 & 5.68462500 \\ \mathrm{H} & 1.76662600 & -2.97581100 & 4.87214000 \\ \mathrm{C} & -0.78564000 & -5.12248300 & 5.40359600 \\ \mathrm{H} & -2.27862200 & -5.71337400 & 3.97545900 \\ \mathrm{H} & 0.79088500 & -4.39505500 & 6.67025400 \\ \mathrm{O} & 6.28660800 & -2.56473400 & 1.27459500 \\ \mathrm{C} & 7.12909600 & -3.70368300 & 1.50008800 \\ \mathrm{H} & 8.12365400 & -3.39989500 & 1.17766900 \\ \mathrm{H} & 7.14843300 & -3.96919300 & 2.56101100 \\ \mathrm{H} & 6.79271600 & -4.55907800 & 0.90694300 \\ \mathrm{~N} & -1.35152000 & -5.96143700 & 6.45893000 \\ \mathrm{O} & -0.80229400 & -5.95380500 & 7.56319200 \\ \mathrm{O} & -2.34919200 & -6.63518300 & 6.19143700\end{array}$




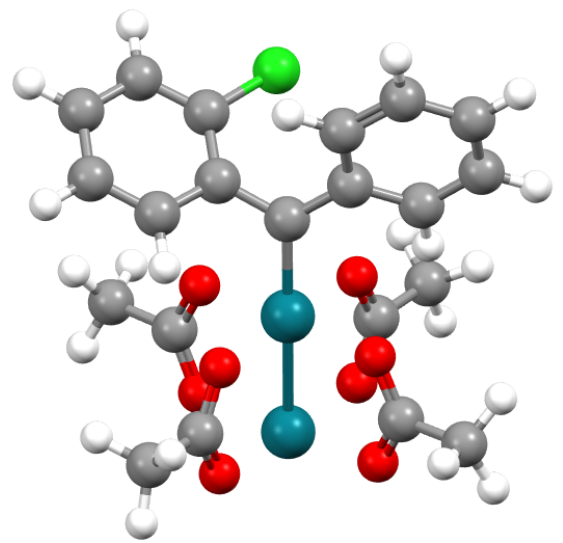

$\begin{array}{lll}\text { Entry } & \text { SCF Energy (a. u.) } \quad \text { Enthalpy (a. u.) } \quad \text { Gibbs Free Energy (a. }\end{array}$ u.)

\begin{tabular}{lccc}
\hline 9e & -2094.24939170 & -2093.82 \\
No imaginary frequency & & \\
Rh & -0.44578100 & -1.44937500 & 1.55752000 \\
O & -1.35161100 & -3.10327400 & 0.69185200 \\
C & 0.86007900 & -2.62004400 & 2.54269300 \\
C & -2.31328400 & -2.95123400 & -0.13941700 \\
C & 2.26668900 & -2.48189000 & 2.17698000 \\
O & -2.80118600 & -1.84969700 & -0.52562800 \\
$\mathrm{C}$ & 2.90467000 & -1.23197200 & 2.32246700 \\
$\mathrm{C}$ & 3.02411800 & -3.53779600 & 1.62583900 \\
$\mathrm{C}$ & 4.24626700 & -1.06741700 & 1.99952200 \\
$\mathrm{H}$ & 2.31731500 & -0.40249800 & 2.69497800 \\
$\mathrm{C}$ & 4.35476100 & -3.36648700 & 1.25661800 \\
$\mathrm{C}$ & 4.97258000 & -2.13269200 & 1.46207500 \\
$\mathrm{H}$ & 4.71972600 & -0.10231100 & 2.14609300 \\
$\mathrm{H}$ & 4.89785900 & -4.19264400 & 0.81249700 \\
$\mathrm{Rh}$ & -2.03511900 & -0.08270000 & 0.24605100 \\
$\mathrm{O}$ & -3.33907300 & -0.23013900 & 1.85989600 \\
$\mathrm{O}$ & -0.60705100 & -0.04333900 & -1.26172900 \\
$\mathrm{O}$ & -1.16980500 & 1.59903000 & 1.09638500 \\
$\mathrm{C}$ & -2.97133300 & -0.84770400 & 2.90284600 \\
$\mathrm{C}$ & 0.48613000 & -0.66411600 & -1.10202700 \\
$\mathrm{C}$ & -0.22148800 & 1.45580300 & 1.92185300 \\
$\mathrm{O}$ & -1.84915700 & -1.43520200 & 3.08028300 \\
$\mathrm{O}$ & 0.83586000 & -1.32989000 & -0.06905800 \\
$\mathrm{O}$ & 0.27736900 & 0.34475100 & 2.31344700 \\
$\mathrm{C}$ & 1.47667900 & -0.65215700 & -2.24216900 \\
$\mathrm{H}$ & 2.49391900 & -0.57072400 & -1.85457900 \\
$\mathrm{H}$ & 1.39875400 & -1.59932600 & -2.78601200 \\
$\mathrm{H}$ & 1.26131900 & 0.16738900 & -2.92792100 \\
$\mathrm{C}$ & -2.90276500 & -4.22594700 & -0.69322800 \\
$\mathrm{H}$ & -3.29223800 & -4.83141200 & 0.13009800 \\
$\mathrm{H}$ & -3.70186200 & -4.00453100 & -1.40002300 \\
$\mathrm{H}$ & -2.11823700 & -4.80588600 & -1.18672700 \\
$\mathrm{C}$ & -3.92265600 & -0.88425300 & 4.07518300 \\
$\mathrm{H}$ & -3.88567100 & -1.86276000 & 4.55765700 \\
$\mathrm{H}$ & -3.60656200 & -0.13552200 & 4.80899900 \\
$\mathrm{H}$ & -4.93805200 & -0.65470900 & 3.75186100 \\
$\mathrm{C}$ & 0.40802700 & 2.69742200 & 2.50596500 \\
$\mathrm{H}$ & 0.46830100 & 2.60478000 & 3.59292000
\end{tabular}




$\begin{array}{lrrr}\mathrm{H} & 1.42927800 & 2.79244100 & 2.12432600 \\ \mathrm{H} & -0.16524300 & 3.58335000 & 2.23418400 \\ \mathrm{C} & 0.49079000 & -3.59889700 & 3.52116800 \\ \mathrm{C} & -0.83137100 & -4.11131300 & 3.59632300 \\ \mathrm{C} & 1.44787800 & -4.05221800 & 4.47121000 \\ \mathrm{C} & -1.16453900 & -5.05067200 & 4.56160300 \\ \mathrm{H} & -1.55825500 & -3.79140100 & 2.86511800 \\ \mathrm{C} & 1.09275600 & -4.95886300 & 5.45704800 \\ \mathrm{H} & 2.45702100 & -3.65869900 & 4.43090600 \\ \mathrm{C} & -0.21159400 & -5.46747600 & 5.49766700 \\ \mathrm{H} & -2.17006600 & -5.45695700 & 4.59510400 \\ \mathrm{H} & 1.82582900 & -5.28086300 & 6.18911000 \\ \mathrm{H} & 6.01408600 & -2.00513400 & 1.18593200 \\ \mathrm{H} & -0.48465300 & -6.18917700 & 6.26132000 \\ \mathrm{Cl} & 2.27374200 & -5.09279600 & 1.27630900\end{array}$

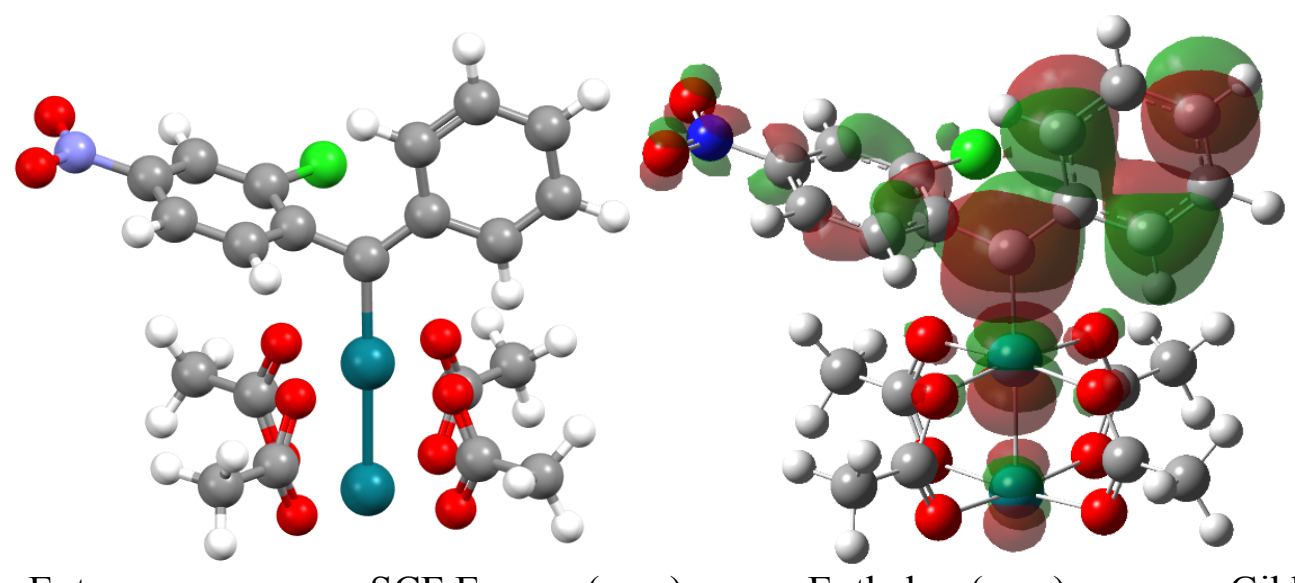

Entry SCF Energy (a. u.) Enthalpy (a. u.) $\quad$ Gibbs Free Energy (a. u.)

\begin{tabular}{lccc}
\hline 9f & \multicolumn{2}{c}{-2298.75279887} & -2298.32 \\
No imaginary frequency & & \\
Rh & -0.44346700 & -1.51961400 & 1.55403500 \\
O & -1.44822700 & -3.16919300 & 0.80550400 \\
C & 0.81579400 & -2.66661400 & 2.62427500 \\
C & -2.38848900 & -3.01404900 & -0.04879000 \\
C & 2.22391200 & -2.39923900 & 2.32467800 \\
O & -2.80180500 & -1.91137500 & -0.51303800 \\
C & 2.87444100 & -1.27943000 & 2.87626400 \\
C & 2.93024800 & -3.19204000 & 1.39919200 \\
C & 4.18124300 & -0.96961800 & 2.52850000 \\
H & 2.32947100 & -0.64612000 & 3.56349900 \\
C & 4.23036000 & -2.89062200 & 1.02414300 \\
C & 4.83765200 & -1.77716700 & 1.60027500 \\
H & 4.68932400 & -0.11478100 & 2.95407700 \\
H & 4.76676900 & -3.49922600 & 0.30922300 \\
Rh & -1.95600300 & -0.14570700 & 0.17342900 \\
O & -3.27589200 & -0.14895300 & 1.77642500 \\
O & -0.52689700 & -0.24598100 & -1.33076500 \\
O & -1.01042800 & 1.52824500 & 0.94986400 \\
C & -2.94125200 & -0.72011900 & 2.85553600 \\
C & 0.53192900 & -0.91383400 & -1.13643200 \\
C & -0.04362700 & 1.37465800 & 1.75345800
\end{tabular}




$\begin{array}{lrrr}\mathrm{O} & -1.84585100 & -1.34539100 & 3.07145300 \\ \mathrm{O} & 0.84223800 & -1.54179400 & -0.06830000 \\ \mathrm{O} & 0.40708900 & 0.26002200 & 2.18866500 \\ \mathrm{C} & 1.52027900 & -1.01777900 & -2.27256200 \\ \mathrm{H} & 2.53540700 & -1.11429400 & -1.88504300 \\ \mathrm{H} & 1.28978500 & -1.91633900 & -2.85474800 \\ \mathrm{H} & 1.43876100 & -0.15006000 & -2.92802400 \\ \mathrm{C} & -3.04453700 & -4.28312700 & -0.53554200 \\ \mathrm{H} & -3.40518300 & -4.85836200 & 0.32126200 \\ \mathrm{H} & -3.87141600 & -4.05509700 & -1.20740300 \\ \mathrm{H} & -2.30322800 & -4.89583300 & -1.05645100 \\ \mathrm{C} & -3.89758100 & -0.65063200 & 4.02132800 \\ \mathrm{H} & -3.95807600 & -1.62391300 & 4.51273000 \\ \mathrm{H} & -3.51318100 & 0.06891800 & 4.75144200 \\ \mathrm{H} & -4.88441000 & -0.32890900 & 3.68911400 \\ \mathrm{C} & 0.68041500 & 2.60928900 & 2.23277300 \\ \mathrm{H} & 0.96340100 & 2.49873500 & 3.28101400 \\ \mathrm{H} & 1.59780600 & 2.72612100 & 1.64626800 \\ \mathrm{H} & 0.05898300 & 3.49423100 & 2.09522200 \\ \mathrm{C} & 0.46897200 & -3.68456900 & 3.55556100 \\ \mathrm{C} & -0.88472000 & -4.09105000 & 3.72207700 \\ \mathrm{C} & 1.47290800 & -4.32614900 & 4.33835900 \\ \mathrm{C} & -1.20797400 & -5.09287600 & 4.62420700 \\ \mathrm{H} & -1.64885300 & -3.62006500 & 3.12282400 \\ \mathrm{C} & 1.13618600 & -5.31296200 & 5.24775700 \\ \mathrm{H} & 2.50720300 & -4.02355600 & 4.22126200 \\ \mathrm{C} & -0.20441300 & -5.69906500 & 5.38878600 \\ \mathrm{H} & -2.23996600 & -5.40626300 & 4.73954000 \\ \mathrm{H} & 1.90513900 & -5.79011900 & 5.84555000 \\ \mathrm{H} & -0.46563100 & -6.47821700 & 6.09818100 \\ \mathrm{Cl} & 2.15216300 & -4.59002300 & 0.68381300 \\ \mathrm{~N} & 6.20708500 & -1.45163200 & 1.21165900 \\ \mathrm{O} & 6.73664500 & -0.46744500 & 1.73217700 \\ \mathrm{O} & 6.76264600 & -2.17671400 & 0.38313800\end{array}$

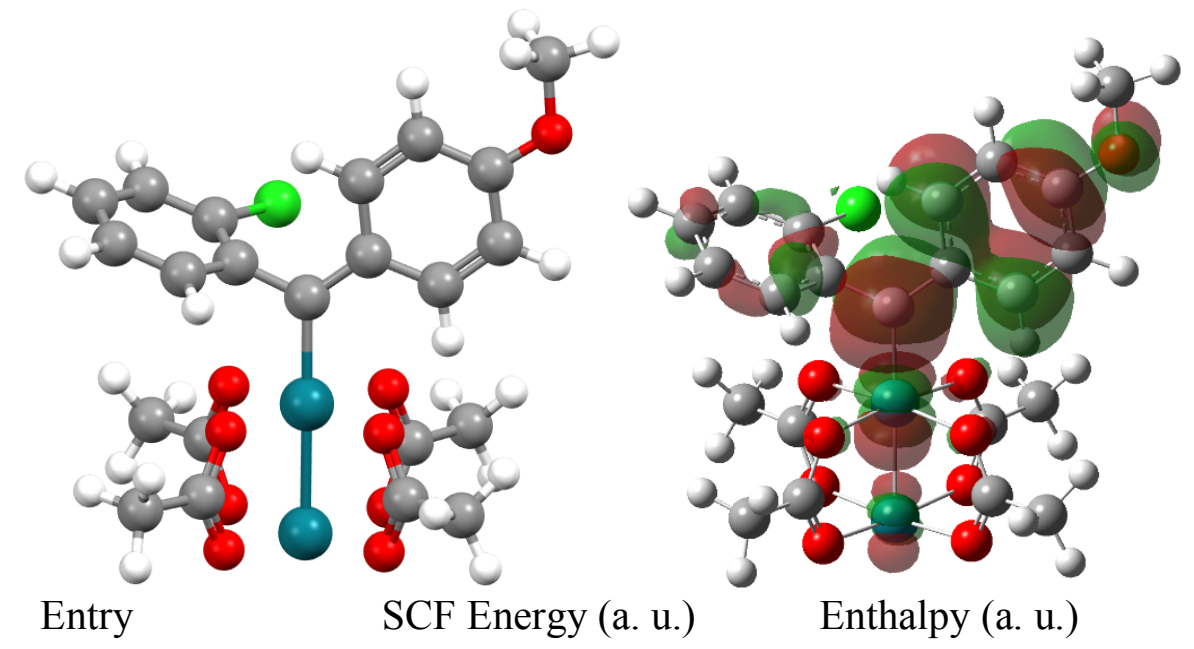

Gibbs Free Energy (a. u.)

\begin{tabular}{|c|c|c|}
\hline $9 \mathrm{~g}$ & -2208.78973999 & -2208.33 \\
\hline \multicolumn{3}{|c|}{ No imaginary frequency } \\
\hline $\mathrm{Rh}$ & -0.51304600 & 1.53890600 \\
\hline
\end{tabular}




\begin{tabular}{|c|c|c|c|}
\hline $\mathrm{O}$ & -1.49564400 & -3.10150900 & 0.90490500 \\
\hline $\mathrm{C}$ & 0.71569200 & -2.45474500 & 2.73757500 \\
\hline $\mathrm{C}$ & -2.43127200 & -3.01606600 & 0.03714200 \\
\hline $\mathrm{C}$ & 2.14513800 & -2.20559800 & 2.50563100 \\
\hline $\mathrm{O}$ & -2.84596700 & -1.95604200 & -0.51642600 \\
\hline $\mathrm{C}$ & 2.74699800 & -1.02882100 & 2.98747600 \\
\hline $\mathrm{C}$ & 2.94205500 & -3.08131200 & 1.75002100 \\
\hline $\mathrm{C}$ & 4.08965300 & -0.75836800 & 2.74056800 \\
\hline $\mathrm{H}$ & 2.13732600 & -0.32849200 & 3.54385500 \\
\hline $\mathrm{C}$ & 4.28006100 & -2.80916200 & 1.47742600 \\
\hline $\mathrm{C}$ & 4.85741900 & -1.64362000 & 1.98031000 \\
\hline $\mathrm{H}$ & 4.53418300 & 0.14982200 & 3.13452900 \\
\hline $\mathrm{H}$ & 4.85857200 & -3.50643400 & 0.88206500 \\
\hline $\mathrm{Rh}$ & -1.98421800 & -0.13485400 & -0.00880100 \\
\hline $\mathrm{O}$ & -3.33018600 & 0.03361200 & 1.56938200 \\
\hline $\mathrm{O}$ & -0.52906300 & -0.40592100 & -1.46614600 \\
\hline $\mathrm{O}$ & -1.02309900 & 1.59546000 & 0.61433300 \\
\hline $\mathrm{C}$ & -3.03560300 & -0.46022900 & 2.69704900 \\
\hline $\mathrm{C}$ & 0.52587600 & -1.04614100 & -1.17635000 \\
\hline $\mathrm{C}$ & -0.09632100 & 1.51181100 & 1.47398100 \\
\hline $\mathrm{O}$ & -1.96061300 & -1.08755500 & 2.99314500 \\
\hline $\mathrm{O}$ & 0.81583100 & -1.55676100 & -0.04274300 \\
\hline $\mathrm{O}$ & 0.31952600 & 0.44439000 & 2.03941000 \\
\hline $\mathrm{C}$ & 1.54109400 & -1.26428100 & -2.27359500 \\
\hline $\mathrm{H}$ & 2.55254100 & -1.21509300 & -1.86655700 \\
\hline $\mathrm{H}$ & 1.39475700 & -2.26540500 & -2.69270900 \\
\hline $\mathrm{H}$ & 1.41098800 & -0.52864700 & -3.06766600 \\
\hline $\mathrm{C}$ & -3.07386000 & -4.32305900 & -0.36296900 \\
\hline $\mathrm{H}$ & -3.28252500 & -4.92205900 & 0.52616800 \\
\hline $\mathrm{H}$ & -3.99086700 & -4.14431400 & -0.92417300 \\
\hline $\mathrm{H}$ & -2.37363400 & -4.88684400 & -0.98751600 \\
\hline $\mathrm{C}$ & -4.02456100 & -0.28799800 & 3.82580500 \\
\hline $\mathrm{H}$ & -4.14249100 & -1.23121300 & 4.36381300 \\
\hline $\mathrm{H}$ & -3.63288600 & 0.45105600 & 4.53204600 \\
\hline $\mathrm{H}$ & -4.98639800 & 0.05500600 & 3.44505800 \\
\hline $\mathrm{C}$ & 0.62267600 & 2.78321200 & 1.85948900 \\
\hline $\mathrm{H}$ & 0.85502800 & 2.77734900 & 2.92593500 \\
\hline $\mathrm{H}$ & 1.56827200 & 2.83153400 & 1.30925100 \\
\hline $\mathrm{H}$ & 0.02130100 & 3.65588100 & 1.60406600 \\
\hline $\mathrm{C}$ & 0.32395300 & -3.41925800 & 3.69199000 \\
\hline $\mathrm{C}$ & -1.04461700 & -3.80323600 & 3.85491900 \\
\hline $\mathrm{C}$ & 1.28722800 & -4.05393000 & 4.53387100 \\
\hline $\mathrm{C}$ & -1.40865900 & -4.75306900 & 4.77802600 \\
\hline $\mathrm{H}$ & -1.79184200 & -3.34423300 & 3.22612900 \\
\hline $\mathrm{C}$ & 0.92896900 & -4.99735800 & 5.47153800 \\
\hline $\mathrm{H}$ & 2.32997300 & -3.77500100 & 4.43647900 \\
\hline $\mathrm{C}$ & -0.43073900 & -5.35946700 & 5.59869100 \\
\hline $\mathrm{H}$ & -2.44188800 & -5.05782100 & 4.90097900 \\
\hline $\mathrm{H}$ & 1.68574700 & -5.45109700 & 6.09783000 \\
\hline $\mathrm{H}$ & 5.90126200 & -1.43098700 & 1.77439000 \\
\hline $\mathrm{Cl}$ & 2.23961100 & -4.55962000 & 1.09578300 \\
\hline $\mathrm{O}$ & -0.88748600 & -6.26493000 & 6.46918900 \\
\hline $\mathrm{C}$ & 0.03801400 & -6.93358300 & 7.33908600 \\
\hline
\end{tabular}




$\begin{array}{lrrr}\mathrm{H} & -0.56564700 & -7.60965200 & 7.94217700 \\ \mathrm{H} & 0.76969900 & -7.50528000 & 6.76125800 \\ \mathrm{H} & 0.54971200 & -6.21589600 & 7.98656500\end{array}$

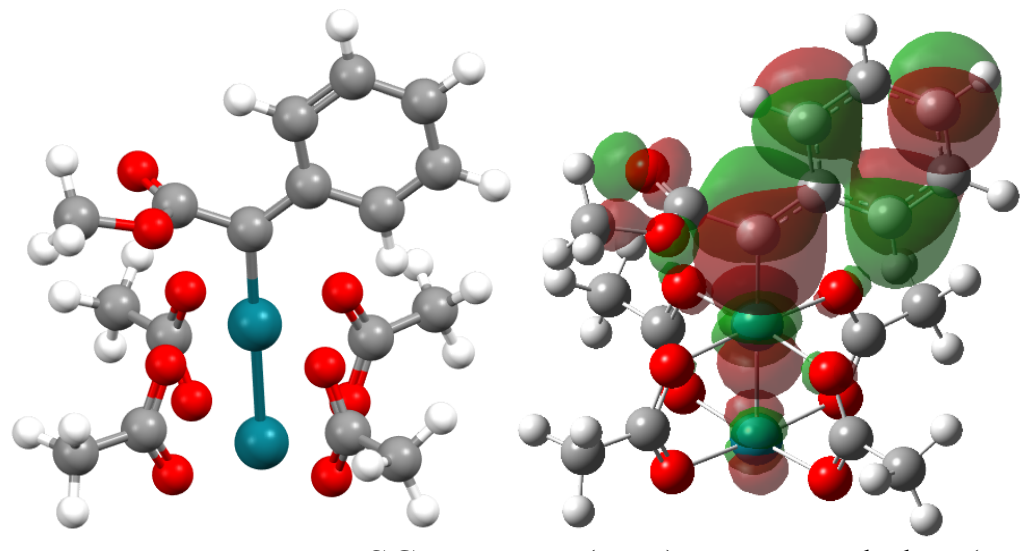

Entry

SCF Energy (a. u.)

Enthalpy (a. u.)

Gibbs Free Energy (a. u.)

\begin{tabular}{|c|c|c|c|}
\hline \multirow{2}{*}{\multicolumn{2}{|c|}{$\begin{array}{l}\mathbf{1 0}-1631 \\
\text { No imaginary frequency }\end{array}$}} & 1.45221519 & \multirow[t]{2}{*}{-1631.05} \\
\hline & & & \\
\hline $\mathrm{Rh}$ & 0.22354300 & 0.12846000 & -0.31808600 \\
\hline $\mathrm{C}$ & -1.48475000 & -0.52599600 & -1.17024200 \\
\hline $\mathrm{C}$ & -1.43360600 & -1.94670400 & -1.57914100 \\
\hline $\mathrm{O}$ & -1.90000000 & -2.75736400 & -0.62252500 \\
\hline $\mathrm{C}$ & -1.68690600 & -4.17008200 & -0.83533200 \\
\hline $\mathrm{H}$ & -2.17548300 & -4.49612300 & -1.75510000 \\
\hline $\mathrm{H}$ & -2.12755400 & -4.66279300 & 0.02912100 \\
\hline $\mathrm{H}$ & -0.61643300 & -4.37753600 & -0.89048200 \\
\hline $\mathrm{O}$ & -0.97460300 & -2.30985800 & -2.64779800 \\
\hline $\mathrm{C}$ & -2.69424100 & 0.19039000 & -1.37410600 \\
\hline $\mathrm{C}$ & -2.83526900 & 1.52621600 & -0.90145700 \\
\hline $\mathrm{C}$ & -4.02954500 & 2.20794100 & -1.07627000 \\
\hline $\mathrm{C}$ & -5.09950800 & 1.59038900 & -1.73409500 \\
\hline $\mathrm{C}$ & -4.98511700 & 0.27855500 & -2.21639900 \\
\hline $\mathrm{C}$ & -3.80371900 & -0.41758500 & -2.03326000 \\
\hline $\mathrm{H}$ & -3.71353600 & -1.43245500 & -2.40494400 \\
\hline $\mathrm{H}$ & -5.82126100 & -0.18860600 & -2.72501800 \\
\hline $\mathrm{H}$ & -6.03029800 & 2.13193000 & -1.87169000 \\
\hline $\mathrm{H}$ & -4.13462200 & 3.22180100 & -0.70539600 \\
\hline $\mathrm{H}$ & -2.00248500 & 1.99040200 & -0.39558300 \\
\hline $\mathrm{O}$ & 0.54023900 & -1.70100400 & 0.59980600 \\
\hline $\mathrm{C}$ & 1.55587400 & -1.86023400 & 1.35886800 \\
\hline $\mathrm{O}$ & 2.41157200 & -0.97597300 & 1.66224200 \\
\hline $\mathrm{Rh}$ & 2.26388100 & 0.91002100 & 0.81459200 \\
\hline $\mathrm{O}$ & 1.05646000 & 1.53959200 & 2.37989300 \\
\hline $\mathrm{C}$ & -0.19460300 & 1.36438300 & 2.30488800 \\
\hline $\mathrm{O}$ & -0.82164400 & 0.81312100 & 1.33572800 \\
\hline $\mathrm{O}$ & 3.33274100 & 0.23477200 & -0.82462600 \\
\hline $\mathrm{C}$ & 2.71204200 & -0.30519800 & -1.78881800 \\
\hline $\mathrm{O}$ & 1.44923000 & -0.48075200 & -1.87043600 \\
\hline $\mathrm{O}$ & 1.97948300 & 2.74230200 & -0.11534100 \\
\hline $\mathrm{C}$ & 0.99114000 & 2.89452100 & -0.89189900 \\
\hline $\mathrm{O}$ & 0.11329100 & 2.00862800 & -1.17985400 \\
\hline $\mathrm{C}$ & 3.52086400 & -0.80704500 & -2.96064700 \\
\hline
\end{tabular}




$\begin{array}{lrrr}\mathrm{H} & 3.43459200 & -1.89633200 & -3.01503200 \\ \mathrm{H} & 3.11552200 & -0.39701700 & -3.88897900 \\ \mathrm{H} & 4.56858100 & -0.52795100 & -2.85251300 \\ \mathrm{C} & 0.81201500 & 4.23498600 & -1.56189600 \\ \mathrm{H} & 0.91997000 & 4.11345500 & -2.64369700 \\ \mathrm{H} & -0.19773600 & 4.60704000 & -1.37043000 \\ \mathrm{H} & 1.55034900 & 4.94882100 & -1.19789900 \\ \mathrm{C} & -1.04803200 & 1.82454000 & 3.46149200 \\ \mathrm{H} & -1.90421200 & 2.39003800 & 3.08671800 \\ \mathrm{H} & -1.43451200 & 0.94758300 & 3.98996700 \\ \mathrm{H} & -0.46363100 & 2.43343500 & 4.15080800 \\ \mathrm{C} & 1.75295100 & -3.25069100 & 1.91244400 \\ \mathrm{H} & 0.78824400 & -3.71535700 & 2.12204200 \\ \mathrm{H} & 2.26460900 & -3.85699200 & 1.15740100 \\ \mathrm{H} & 2.36842500 & -3.21994600 & 2.81182400\end{array}$




\section{Rh TS structures:}

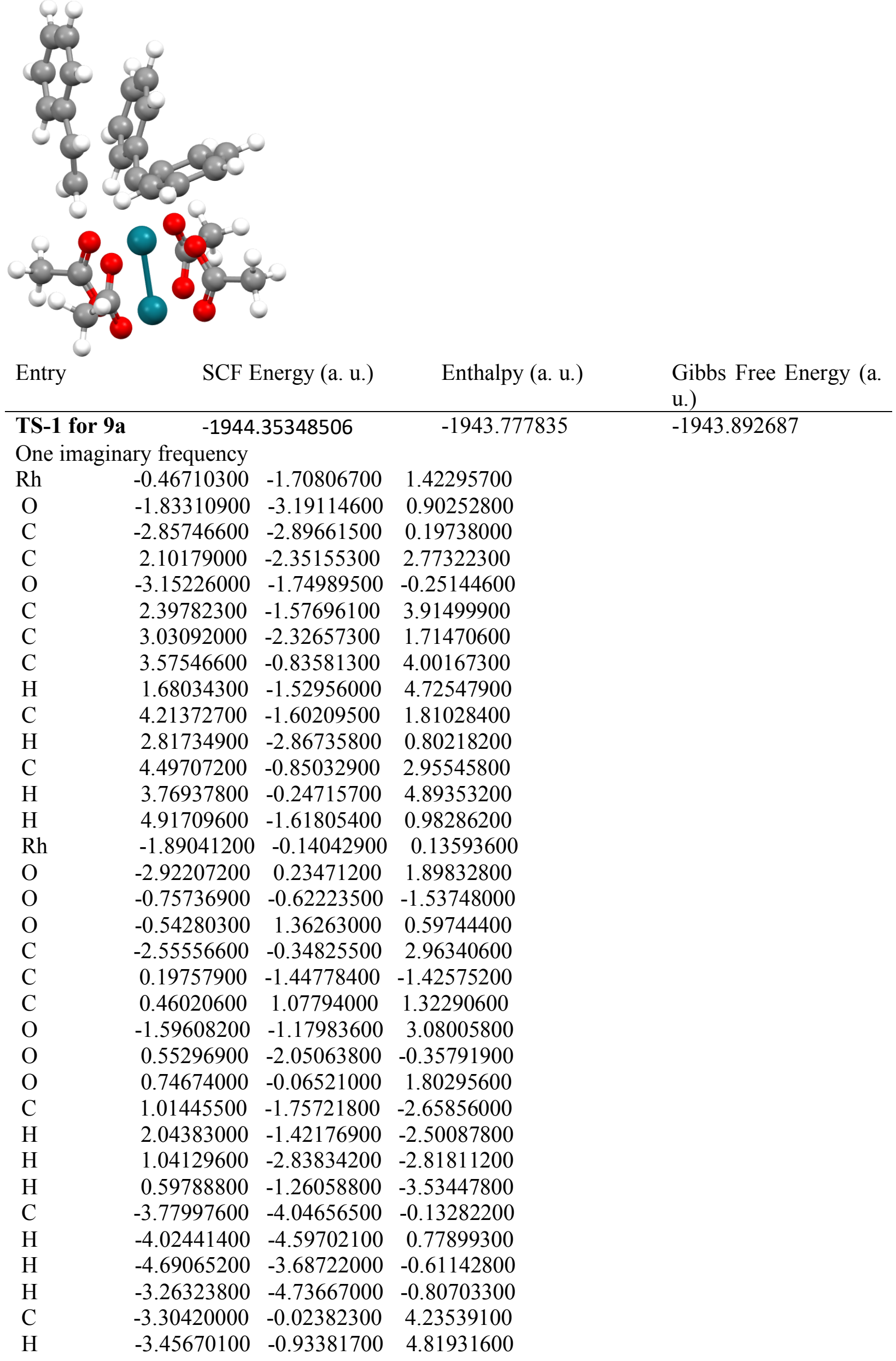




$\begin{array}{lrrr}\mathrm{H} & -2.70032300 & 0.66295800 & 4.83744700 \\ \mathrm{H} & -4.25977300 & 0.44975800 & 4.00969100 \\ \mathrm{C} & 1.39886800 & 2.20601100 & 1.68500800 \\ \mathrm{H} & 1.00851600 & 2.71709600 & 2.57158000 \\ \mathrm{H} & 2.38987100 & 1.81537300 & 1.91916000 \\ \mathrm{H} & 1.45298000 & 2.93070000 & 0.87106400 \\ \mathrm{C} & 0.18329300 & -3.62788600 & 3.83339300 \\ \mathrm{C} & -1.19964900 & -3.91665400 & 3.88280000 \\ \mathrm{C} & 0.97400500 & -4.04548000 & 4.93044500 \\ \mathrm{C} & -1.76798200 & -4.52319300 & 4.99782000 \\ \mathrm{H} & -1.81772400 & -3.65571900 & 3.03609900 \\ \mathrm{C} & 0.40404500 & -4.64963500 & 6.04551800 \\ \mathrm{H} & 2.04569000 & -3.89756200 & 4.89966400 \\ \mathrm{C} & -0.97210800 & -4.88132000 & 6.08875800 \\ \mathrm{H} & -2.83484300 & -4.72440700 & 5.01386600 \\ \mathrm{H} & 1.03639600 & -4.95948500 & 6.87112400 \\ \mathrm{H} & 5.41979400 & -0.28283400 & 3.02606800 \\ \mathrm{H} & -1.41815100 & -5.35603100 & 6.95748500 \\ \mathrm{C} & 0.76402700 & -2.97022200 & 2.65525300 \\ \mathrm{C} & 0.84403500 & -4.59361000 & 1.23698900 \\ \mathrm{C} & 1.80234100 & -5.33106900 & 1.89121400 \\ \mathrm{H} & 1.10893100 & -3.99531400 & 0.37547800 \\ \mathrm{H} & -0.20877800 & -4.83041600 & 1.31730800 \\ \mathrm{H} & 2.84563800 & -5.09203100 & 1.70121000 \\ \mathrm{C} & 1.56545800 & -6.29726400 & 2.93309000 \\ \mathrm{C} & 2.64974300 & -6.70611600 & 3.74121300 \\ \mathrm{C} & 0.27891500 & -6.80025400 & 3.22346700 \\ \mathrm{C} & 2.45029600 & -7.55875500 & 4.81968700 \\ \mathrm{H} & 3.64276700 & -6.32499300 & 3.52137900 \\ \mathrm{C} & 0.08304500 & -7.64906000 & 4.30373800 \\ \mathrm{H} & -0.56269500 & -6.51767500 & 2.60330800 \\ \mathrm{C} & 1.16364300 & -8.02422700 & 5.10902300 \\ \mathrm{H} & 3.28947600 & -7.85521700 & 5.44064600 \\ \mathrm{H} & -0.91305300 & -8.01632400 & 4.52711800 \\ \mathrm{H} & 1.00425700 & -8.68467800 & 5.95567400\end{array}$




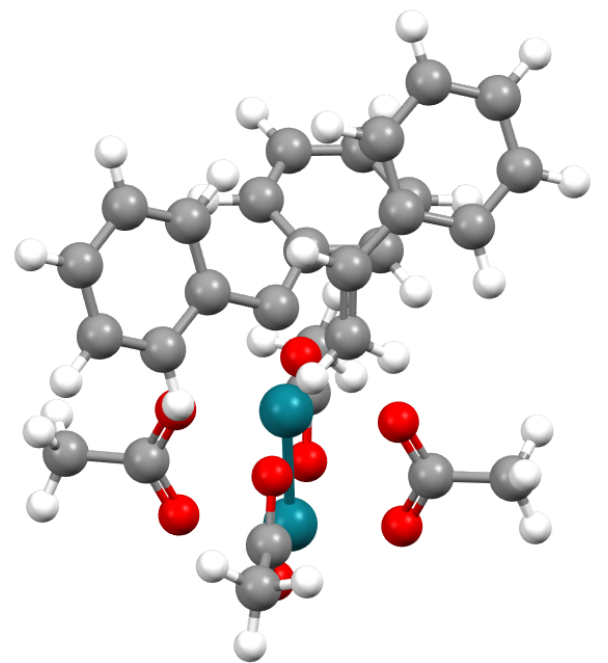

Entry SCF Energy (a. u.) $\quad$ Enthalpy (a. u.) $\quad$ Gibbs Free Energy (a. u.)

\begin{tabular}{lrcc}
\hline TS-2 for 9a & -1944.34767444 & -1943.77 \\
One imaginary frequency & & \\
Rh & -0.48092400 & -1.68547500 & 1.37808700 \\
O & -1.81458200 & -3.21107300 & 0.90459300 \\
$\mathrm{C}$ & -2.84957400 & -2.95467600 & 0.20083600 \\
$\mathrm{C}$ & 2.07172700 & -2.22015900 & 2.82912300 \\
$\mathrm{O}$ & -3.16901800 & -1.82507300 & -0.27549500 \\
$\mathrm{C}$ & 2.41207200 & -1.58188100 & 4.04270800 \\
$\mathrm{C}$ & 2.91464000 & -1.99156000 & 1.72120400 \\
$\mathrm{C}$ & 3.55427400 & -0.79014400 & 4.14533800 \\
$\mathrm{H}$ & 1.75627600 & -1.67174200 & 4.89951500 \\
$\mathrm{C}$ & 4.07307500 & -1.23413900 & 1.83476500 \\
$\mathrm{H}$ & 2.62933500 & -2.38614700 & 0.75741000 \\
$\mathrm{C}$ & 4.40457500 & -0.62875900 & 3.05040200 \\
$\mathrm{H}$ & 3.78059900 & -0.30176800 & 5.08856000 \\
$\mathrm{H}$ & 4.71164900 & -1.10160500 & 0.96661500 \\
$\mathrm{Rh}$ & -1.93865700 & -0.18207700 & 0.06268700 \\
$\mathrm{O}$ & -2.96928100 & 0.22303700 & 1.81557200 \\
$\mathrm{O}$ & -0.80933200 & -0.68212700 & -1.60728300 \\
$\mathrm{O}$ & -0.62112400 & 1.36024600 & 0.47702700 \\
$\mathrm{C}$ & -2.57868100 & -0.31481300 & 2.89652100 \\
$\mathrm{C}$ & 0.15868800 & -1.49065100 & -1.48399100 \\
$\mathrm{C}$ & 0.38877200 & 1.11577900 & 1.20858700 \\
$\mathrm{O}$ & -1.60379700 & -1.12503900 & 3.02876400 \\
$\mathrm{O}$ & 0.53008300 & -2.06122700 & -0.40420800 \\
$\mathrm{O}$ & 0.70002700 & -0.00873100 & 1.71438000 \\
$\mathrm{C}$ & 0.97005200 & -1.81913600 & -2.71508500 \\
$\mathrm{H}$ & 1.99664500 & -1.46876200 & -2.57245900 \\
$\mathrm{H}$ & 1.00775900 & -2.90341500 & -2.84933000 \\
$\mathrm{H}$ & 0.54174300 & -1.34806800 & -3.59938200 \\
$\mathrm{C}$ & -3.75599100 & -4.12795300 & -0.08857800 \\
$\mathrm{H}$ & -4.00384200 & -4.63970500 & 0.84478200 \\
$\mathrm{H}$ & -4.66606800 & -3.79993200 & -0.59016400 \\
$\mathrm{H}$ & -3.22546600 & -4.84212300 & -0.72576100 \\
$\mathrm{C}$ & -3.31534900 & 0.04673700 & 4.16507200 \\
$\mathrm{H}$ & -3.39437300 & -0.82773500 & 4.81362400 \\
$\mathrm{H}$ & -2.74366900 & 0.81255400 & 4.69975600
\end{tabular}




$\begin{array}{lrrr}\mathrm{H} & -4.30432600 & 0.44382600 & 3.93506400 \\ \mathrm{C} & 1.30115400 & 2.27381900 & 1.54103500 \\ \mathrm{H} & 0.89075300 & 2.80796100 & 2.40462200 \\ \mathrm{H} & 2.29704900 & 1.91151300 & 1.79860400 \\ \mathrm{H} & 1.34988700 & 2.97081000 & 0.70280900 \\ \mathrm{C} & 0.18595100 & -3.58783800 & 3.86434700 \\ \mathrm{C} & -1.19781100 & -3.84334200 & 3.91364000 \\ \mathrm{C} & 0.97233400 & -4.05547400 & 4.94081600 \\ \mathrm{C} & -1.77379900 & -4.48545300 & 5.00868200 \\ \mathrm{H} & -1.81756500 & -3.53956100 & 3.08344400 \\ \mathrm{C} & 0.39918300 & -4.69876100 & 6.03319600 \\ \mathrm{H} & 2.04844900 & -3.93171300 & 4.90664100 \\ \mathrm{C} & -0.98222600 & -4.90794100 & 6.07727900 \\ \mathrm{H} & -2.84524400 & -4.66182800 & 5.02239800 \\ \mathrm{H} & 1.03108700 & -5.04819100 & 6.84407000 \\ \mathrm{H} & 5.30384400 & -0.02666400 & 3.13747400 \\ \mathrm{H} & -1.43208600 & -5.41009000 & 6.92844400 \\ \mathrm{C} & 0.79064200 & -2.92871100 & 2.67286800 \\ \mathrm{C} & 0.93375400 & -4.48334900 & 1.31753900 \\ \mathrm{C} & 1.88316400 & -5.17566100 & 2.04855300 \\ \mathrm{H} & 1.19547700 & -3.96835400 & 0.40197900 \\ \mathrm{H} & -0.10989800 & -4.75793800 & 1.40010000 \\ \mathrm{H} & 1.53853500 & -5.72616500 & 2.91916300 \\ \mathrm{C} & 3.31195100 & -5.11638400 & 1.87713500 \\ \mathrm{C} & 3.91741700 & -4.71885100 & 0.66522300 \\ \mathrm{C} & 4.14039100 & -5.42674100 & 2.97746300 \\ \mathrm{C} & 5.29928400 & -4.61614200 & 0.56783800 \\ \mathrm{H} & 3.29797600 & -4.51021400 & -0.20020400 \\ \mathrm{C} & 5.52141700 & -5.30195300 & 2.88300200 \\ \mathrm{H} & 3.68247600 & -5.74595400 & 3.90894400 \\ \mathrm{C} & 6.10369300 & -4.89454600 & 1.67812900 \\ \mathrm{H} & 5.75412400 & -4.31555700 & -0.37040600 \\ \mathrm{H} & 6.14644200 & -5.52435700 & 3.74174500 \\ \mathrm{H} & 7.18261000 & -4.80368400 & 1.60155300 \\ & & & \\ & & & \end{array}$




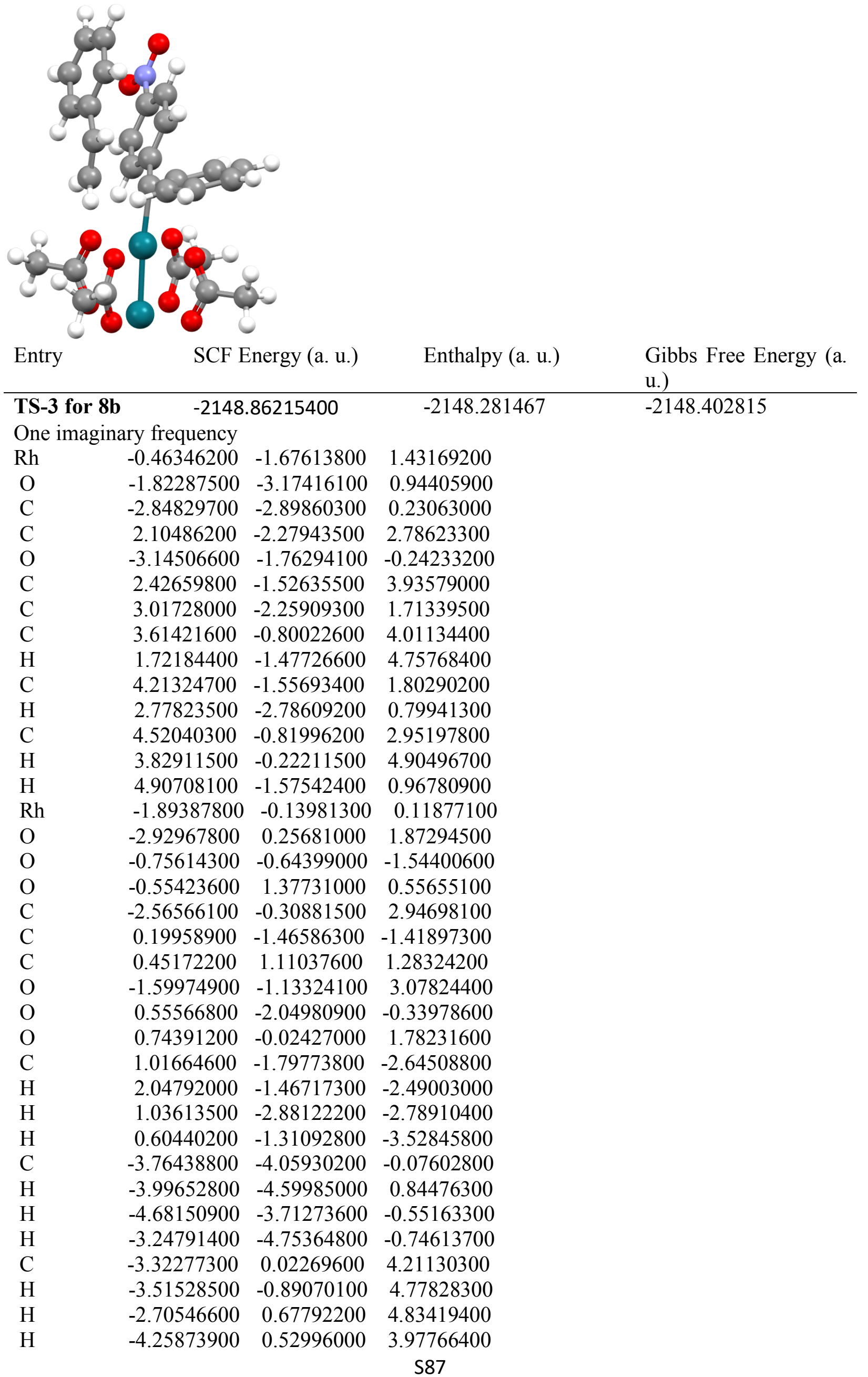




$\begin{array}{lrrr}\mathrm{C} & 1.38800700 & 2.24660100 & 1.62303000 \\ \mathrm{H} & 0.99410500 & 2.77717400 & 2.49640800 \\ \mathrm{H} & 2.37874900 & 1.86238500 & 1.86854400 \\ \mathrm{H} & 1.44352100 & 2.95251900 & 0.79286500 \\ \mathrm{C} & 0.19624400 & -3.56228300 & 3.84395500 \\ \mathrm{C} & -1.18319800 & -3.86635600 & 3.89754200 \\ \mathrm{C} & 1.01232400 & -4.01478100 & 4.90755700 \\ \mathrm{C} & -1.72825600 & -4.55208100 & 4.97360700 \\ \mathrm{H} & -1.81903800 & -3.57202300 & 3.07644000 \\ \mathrm{C} & 0.48116500 & -4.69702700 & 5.99230700 \\ \mathrm{H} & 2.07964700 & -3.84565600 & 4.87219900 \\ \mathrm{C} & -0.88588900 & -4.95745800 & 6.00738700 \\ \mathrm{H} & -2.78221200 & -4.79309800 & 5.01200100 \\ \mathrm{H} & 1.11006600 & -5.05790800 & 6.79417600 \\ \mathrm{H} & 5.45181900 & -0.26619100 & 3.01541100 \\ \mathrm{C} & 0.75854900 & -2.86745100 & 2.67450600 \\ \mathrm{C} & 0.85330600 & -4.62085100 & 1.25103200 \\ \mathrm{C} & 1.78905000 & -5.34491500 & 1.93846500 \\ \mathrm{H} & 1.13612600 & -4.00892600 & 0.40520400 \\ \mathrm{H} & -0.20642000 & -4.82438900 & 1.33407400 \\ \mathrm{H} & 2.83822200 & -5.11319700 & 1.77038300 \\ \mathrm{C} & 1.51983000 & -6.30637900 & 2.98291600 \\ \mathrm{C} & 2.57497700 & -6.69920500 & 3.83577500 \\ \mathrm{C} & 0.22812200 & -6.82024300 & 3.22749100 \\ \mathrm{C} & 2.33996100 & -7.53819900 & 4.91875400 \\ \mathrm{H} & 3.57301500 & -6.31334500 & 3.65011000 \\ \mathrm{C} & -0.00276200 & -7.65839500 & 4.30984600 \\ \mathrm{H} & -0.59104000 & -6.55574200 & 2.56961800 \\ \mathrm{C} & 1.04723000 & -8.00982600 & 5.16585700 \\ \mathrm{H} & 3.15660900 & -7.81722800 & 5.57650800 \\ \mathrm{H} & -1.00341100 & -8.03401500 & 4.49736000 \\ \mathrm{H} & 0.85771000 & -8.65366600 & 6.01870900 \\ \mathrm{~N} & -1.44546000 & -5.72848200 & 7.11882200 \\ \mathrm{O} & -0.67554900 & -6.11919800 & 7.99876300 \\ \mathrm{O} & -2.65698900 & -5.95582200 & 7.11377900 \\ & & & \\ & & & \\ & & & \end{array}$




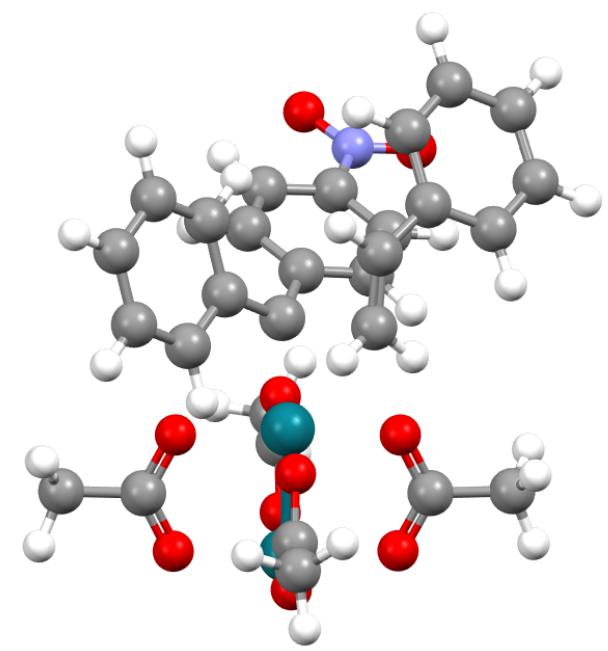

Entry SCF Energy (a. u.) Enthalpy (a. u.) Gibbs Free Energy (a. u.)

\begin{tabular}{crcc}
\hline TS-4 for 8b & -2148.85296059 & -2148.27 \\
One imaginary frequency & & \\
$\mathrm{Rh}$ & -0.47571900 & -1.68816900 & 1.41176200 \\
$\mathrm{O}$ & -1.80545000 & -3.21088600 & 0.92848300 \\
$\mathrm{C}$ & -2.82799500 & -2.95639400 & 0.20500500 \\
$\mathrm{C}$ & 2.03025400 & -2.23624400 & 2.88272000 \\
$\mathrm{O}$ & -3.12669400 & -1.83266300 & -0.29742800 \\
$\mathrm{C}$ & 2.37754400 & -1.59444700 & 4.09445900 \\
$\mathrm{C}$ & 2.88596500 & -2.05266500 & 1.77438100 \\
$\mathrm{C}$ & 3.54830800 & -0.85743300 & 4.20902000 \\
$\mathrm{H}$ & 1.71055700 & -1.65060800 & 4.94463500 \\
$\mathrm{C}$ & 4.07285800 & -1.35221500 & 1.88304100 \\
$\mathrm{H}$ & 2.58811300 & -2.44229800 & 0.81379700 \\
$\mathrm{C}$ & 4.39874200 & -0.76607600 & 3.10818800 \\
$\mathrm{H}$ & 3.81318400 & -0.36672600 & 5.13634100 \\
$\mathrm{H}$ & 4.73840700 & -1.24104200 & 1.03770200 \\
$\mathrm{Rh}$ & -1.89095700 & -0.19631100 & 0.04395700 \\
$\mathrm{O}$ & -2.95726300 & 0.24054300 & 1.76584200 \\
$\mathrm{O}$ & -0.73013900 & -0.72452800 & -1.59412700 \\
$\mathrm{O}$ & -0.56598300 & 1.33871800 & 0.46424100 \\
$\mathrm{C}$ & -2.60195900 & -0.29044300 & 2.86139600 \\
$\mathrm{C}$ & 0.23342700 & -1.53146800 & -1.43771700 \\
$\mathrm{C}$ & 0.42875100 & 1.09842900 & 1.21550500 \\
$\mathrm{O}$ & -1.63600900 & -1.10713200 & 3.02632500 \\
$\mathrm{O}$ & 0.57741300 & -2.08731400 & -0.34015500 \\
$\mathrm{O}$ & 0.71624000 & -0.02060200 & 1.75143200 \\
$\mathrm{C}$ & 1.07484000 & -1.87922600 & -2.64224600 \\
$\mathrm{H}$ & 2.09836300 & -1.52951900 & -2.47775200 \\
$\mathrm{H}$ & 1.11267000 & -2.96541800 & -2.75994300 \\
$\mathrm{H}$ & 0.67067600 & -1.41942700 & -3.54359500 \\
$\mathrm{C}$ & -3.75985700 & -4.11729900 & -0.04839900 \\
$\mathrm{H}$ & -4.24244400 & -4.39851500 & 0.89278400 \\
$\mathrm{H}$ & -4.52081900 & -3.84976800 & -0.78099900 \\
$\mathrm{H}$ & -3.18791500 & -4.98074700 & -0.39681100 \\
$\mathrm{C}$ & -3.37034700 & 0.08369600 & 4.10652300 \\
$\mathrm{H}$ & -3.50274500 & -0.79478800 & 4.74102300 \\
$\mathrm{H}$ & -2.79141600 & 0.82191400 & 4.67113000
\end{tabular}




$\begin{array}{lrrr}\mathrm{H} & -4.33623600 & 0.51676500 & 3.84609000 \\ \mathrm{C} & 1.35593900 & 2.24613900 & 1.53938200 \\ \mathrm{H} & 1.02516300 & 2.71243800 & 2.47350300 \\ \mathrm{H} & 2.37329300 & 1.87918400 & 1.68411700 \\ \mathrm{H} & 1.32645200 & 2.99646500 & 0.74852400 \\ \mathrm{C} & 0.12946000 & -3.58370500 & 3.89388000 \\ \mathrm{C} & -1.25844000 & -3.82897400 & 3.93757700 \\ \mathrm{C} & 0.91436200 & -4.07192300 & 4.96457400 \\ \mathrm{C} & -1.83805400 & -4.48240500 & 5.02236800 \\ \mathrm{H} & -1.87396200 & -3.50802400 & 3.11099200 \\ \mathrm{C} & 0.33565100 & -4.72589100 & 6.04609600 \\ \mathrm{H} & 1.99113000 & -3.95253500 & 4.93587100 \\ \mathrm{C} & -1.04785800 & -4.92579900 & 6.08407100 \\ \mathrm{H} & -2.91035600 & -4.65170700 & 5.03490700 \\ \mathrm{H} & 0.96281800 & -5.09024500 & 6.85364200 \\ \mathrm{H} & -1.50148200 & -5.43730900 & 6.92746200 \\ \mathrm{C} & 0.73075900 & -2.90160000 & 2.72926100 \\ \mathrm{C} & 0.91162300 & -4.57361300 & 1.33930900 \\ \mathrm{C} & 1.91336400 & -5.18025300 & 2.05844300 \\ \mathrm{H} & 1.11122700 & -4.05054100 & 0.41254500 \\ \mathrm{H} & -0.12236600 & -4.84532100 & 1.50327700 \\ \mathrm{H} & 1.63400800 & -5.70748000 & 2.96667100 \\ \mathrm{C} & 3.33283800 & -5.05879700 & 1.81511700 \\ \mathrm{C} & 3.85769900 & -4.72580800 & 0.54843900 \\ \mathrm{C} & 4.22199200 & -5.21025900 & 2.89912000 \\ \mathrm{C} & 5.22154200 & -4.51970600 & 0.38308900 \\ \mathrm{H} & 3.18891600 & -4.63591500 & -0.30105800 \\ \mathrm{C} & 5.58385800 & -4.98152100 & 2.73534700 \\ \mathrm{H} & 3.82737700 & -5.48251600 & 3.87349100 \\ \mathrm{C} & 6.08569700 & -4.63207700 & 1.47801200 \\ \mathrm{H} & 5.61548300 & -4.26583200 & -0.59559400 \\ \mathrm{H} & 6.25548500 & -5.07670700 & 3.58226300 \\ \mathrm{H} & 7.14906300 & -4.45742400 & 1.34865400 \\ \mathrm{~N} & 5.64518700 & -0.02509500 & 3.23238000 \\ \mathrm{O} & 6.39456100 & 0.02368300 & 2.25152400 \\ \mathrm{O} & 5.89685100 & 0.51908200 & 4.31239900\end{array}$




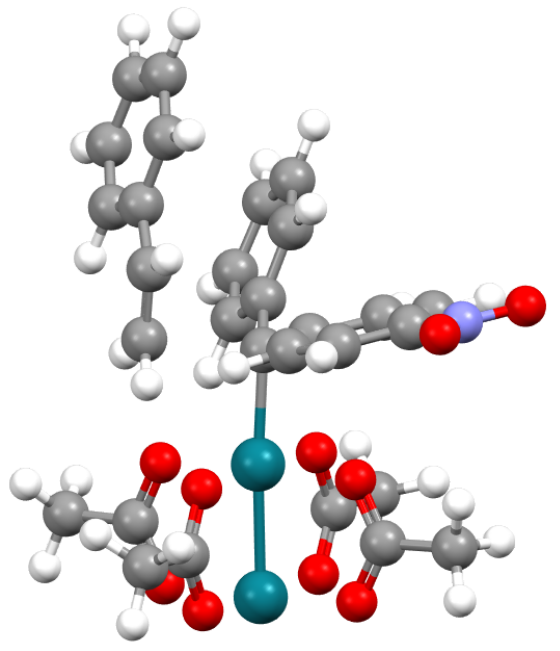

Entry

SCF Energy (a. u.)

Enthalpy (a. u.)

Gibbs Free Energy (a. u.)

\begin{tabular}{lrrc}
\hline TS-5 for 8b & -2148.85950840 & -2148.2787 \\
One imaginary frequency & & \\
Rh & -0.45852800 & -1.68896000 & 1.44267300 \\
$\mathrm{O}$ & -1.81469800 & -3.17692600 & 0.91795900 \\
$\mathrm{C}$ & -2.84021500 & -2.88701500 & 0.21163200 \\
$\mathrm{C}$ & 2.07505400 & -2.33067000 & 2.78737500 \\
$\mathrm{O}$ & -3.12820300 & -1.74520300 & -0.25339300 \\
$\mathrm{C}$ & 2.36204900 & -1.53256600 & 3.91976000 \\
$\mathrm{C}$ & 3.00756500 & -2.33065500 & 1.72825400 \\
$\mathrm{C}$ & 3.52856300 & -0.78704800 & 4.00193200 \\
$\mathrm{H}$ & 1.64265000 & -1.47666200 & 4.72687100 \\
$\mathrm{C}$ & 4.18303900 & -1.60372500 & 1.80455500 \\
$\mathrm{H}$ & 2.79544300 & -2.88772700 & 0.82728800 \\
$\mathrm{C}$ & 4.43545300 & -0.83572600 & 2.94461000 \\
$\mathrm{H}$ & 3.74369000 & -0.17674900 & 4.86929200 \\
$\mathrm{H}$ & 4.90122000 & -1.61304800 & 0.99513000 \\
$\mathrm{Rh}$ & -1.86722100 & -0.13741000 & 0.13036000 \\
$\mathrm{O}$ & -2.91455300 & 0.25298500 & 1.87832700 \\
$\mathrm{O}$ & -0.72083500 & -0.63145900 & -1.52814900 \\
$\mathrm{O}$ & -0.52094300 & 1.36759100 & 0.58945100 \\
$\mathrm{C}$ & -2.56668200 & -0.32789900 & 2.95012500 \\
$\mathrm{C}$ & 0.23914900 & -1.44799800 & -1.39892000 \\
$\mathrm{C}$ & 0.47426100 & 1.09266600 & 1.32747700 \\
$\mathrm{O}$ & -1.61168800 & -1.16416500 & 3.08117300 \\
$\mathrm{O}$ & 0.58874100 & -2.03535400 & -0.31970200 \\
$\mathrm{O}$ & 0.75146900 & -0.04589600 & 1.82756100 \\
$\mathrm{C}$ & 1.06824600 & -1.76908600 & -2.61970600 \\
$\mathrm{H}$ & 2.10038100 & -1.44993800 & -2.44795200 \\
$\mathrm{H}$ & 1.08002900 & -2.85071800 & -2.77848000 \\
$\mathrm{H}$ & 0.67036400 & -1.26715400 & -3.50117300 \\
$\mathrm{C}$ & -3.78755700 & -4.02893200 & -0.06948900 \\
$\mathrm{H}$ & -4.25998400 & -4.33930100 & 0.86763100 \\
$\mathrm{H}$ & -4.55543700 & -3.72807200 & -0.78164400 \\
$\mathrm{H}$ & -3.22892500 & -4.88465000 & -0.45659800 \\
$\mathrm{C}$ & -3.33053500 & 0.00231300 & 4.21062400 \\
$\mathrm{H}$ & -3.50642000 & -0.90775000 & 4.78785200 \\
$\mathrm{H}$ & -2.72586900 & 0.67691800 & 4.82539700 \\
$\mathrm{H}$ & -4.27498600 & 0.49092300 & 3.97103800
\end{tabular}




$\begin{array}{lrrr}\mathrm{C} & 1.41476400 & 2.22018400 & 1.68296200 \\ \mathrm{H} & 1.05066400 & 2.70705800 & 2.59395000 \\ \mathrm{H} & 2.41552200 & 1.83236900 & 1.87769500 \\ \mathrm{H} & 1.43815900 & 2.96206500 & 0.88362500 \\ \mathrm{C} & 0.17783600 & -3.62909800 & 3.83148700 \\ \mathrm{C} & -1.20656100 & -3.92020800 & 3.89036100 \\ \mathrm{C} & 0.98777200 & -4.06963800 & 4.90850200 \\ \mathrm{C} & -1.75615500 & -4.55384200 & 4.99777200 \\ \mathrm{H} & -1.83397500 & -3.63947900 & 3.05711700 \\ \mathrm{C} & 0.43349600 & -4.69855000 & 6.01601500 \\ \mathrm{H} & 2.05817800 & -3.91362500 & 4.86828000 \\ \mathrm{C} & -0.94219200 & -4.93419600 & 6.06881000 \\ \mathrm{H} & -2.82176500 & -4.75860400 & 5.02620600 \\ \mathrm{H} & 1.07531500 & -5.02549300 & 6.82701600 \\ \mathrm{C} & 0.73641600 & -2.92972500 & 2.68466600 \\ \mathrm{C} & 0.84899200 & -4.66321600 & 1.18144000 \\ \mathrm{C} & 1.80580200 & -5.37431800 & 1.84703000 \\ \mathrm{H} & 1.10415500 & -4.03609600 & 0.33764100 \\ \mathrm{H} & -0.20732600 & -4.86608000 & 1.29842900 \\ \mathrm{H} & 2.85009600 & -5.14950200 & 1.64315700 \\ \mathrm{C} & 1.56846300 & -6.32361300 & 2.91094200 \\ \mathrm{C} & 2.64949600 & -6.71150400 & 3.73205600 \\ \mathrm{C} & 0.28470600 & -6.83181100 & 3.20030600 \\ \mathrm{C} & 2.44925900 & -7.54926900 & 4.82279000 \\ \mathrm{H} & 3.64156600 & -6.32743800 & 3.51283000 \\ \mathrm{C} & 0.08784200 & -7.66728800 & 4.29102400 \\ \mathrm{H} & -0.55496100 & -6.56683600 & 2.56933500 \\ \mathrm{C} & 1.16514000 & -8.02166300 & 5.11006000 \\ \mathrm{H} & 3.28678400 & -7.82916500 & 5.45360400 \\ \mathrm{H} & -0.90677300 & -8.04034600 & 4.51165300 \\ \mathrm{H} & 1.00502800 & -8.67128100 & 5.96487100 \\ \mathrm{~N} & 5.66511600 & -0.06447400 & 3.02641000 \\ \mathrm{O} & 6.45726500 & -0.12606800 & 2.08016600 \\ \mathrm{O} & 5.86077300 & 0.61777500 & 4.03783300 \\ \mathrm{H} & -1.37566400 & -5.42937000 & 6.93226700 \\ & & & \\ & & \end{array}$




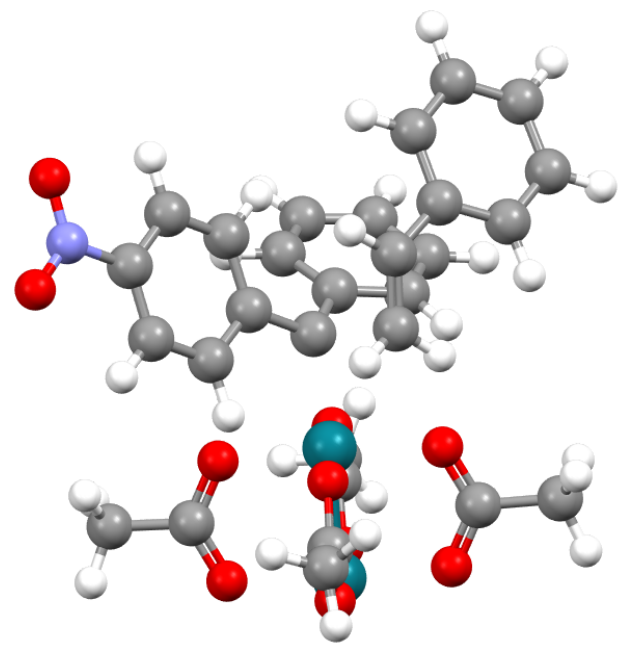

Entry SCF Energy (a. u.) Enthalpy (a. u.) $\quad$ Gibbs Free Energy (a.

\begin{tabular}{|c|c|c|c|c|}
\hline \multirow[b]{2}{*}{ TS-6 for $8 b$} & \multirow{2}{*}{\multicolumn{2}{|c|}{-2148.85643959}} & & \multirow{2}{*}{ u.) } \\
\hline & & & -2148.275532 & \\
\hline \multicolumn{5}{|c|}{ One imaginary frequency } \\
\hline $\mathrm{Rh}$ & -0.48004900 & -1.67909300 & 1.38499700 & \\
\hline $\mathrm{O}$ & -1.81476500 & -3.20854100 & 0.93225300 & \\
\hline $\mathrm{C}$ & -2.85340500 & -2.95787000 & 0.22977900 & \\
\hline $\mathrm{C}$ & 2.06119300 & -2.20854700 & 2.85328200 & \\
\hline $\mathrm{O}$ & -3.16448000 & -1.83635300 & -0.26870900 & \\
\hline $\mathrm{C}$ & 2.43728700 & -1.62619800 & 4.08539600 & \\
\hline $\mathrm{C}$ & 2.89663300 & -1.97310400 & 1.74043600 & \\
\hline $\mathrm{C}$ & 3.60817800 & -0.88019300 & 4.19897600 & \\
\hline $\mathrm{H}$ & 1.78717400 & -1.71747700 & 4.94659600 & \\
\hline $\mathrm{C}$ & 4.08470100 & -1.26605700 & 1.86609300 & \\
\hline $\mathrm{H}$ & 2.58365800 & -2.32968300 & 0.77016700 & \\
\hline $\mathrm{H}$ & 3.86325300 & -0.43008300 & 5.15349400 & \\
\hline $\mathrm{H}$ & 4.72041100 & -1.12967200 & 0.99688000 & \\
\hline $\mathrm{Rh}$ & -1.93111200 & -0.19082700 & 0.04535900 & \\
\hline $\mathrm{O}$ & -2.96458400 & 0.24097300 & 1.78958000 & \\
\hline $\mathrm{O}$ & -0.80042800 & -0.71783800 & -1.61454300 & \\
\hline $\mathrm{O}$ & -0.60669700 & 1.35102800 & 0.44004000 & \\
\hline $\mathrm{C}$ & -2.58412400 & -0.28698100 & 2.87765600 & \\
\hline $\mathrm{C}$ & 0.16630800 & -1.52457500 & -1.47781800 & \\
\hline $\mathrm{C}$ & 0.40131400 & 1.11457400 & 1.17531900 & \\
\hline $\mathrm{O}$ & -1.61036500 & -1.09864000 & 3.02371400 & \\
\hline $\mathrm{O}$ & 0.53481700 & -2.07898800 & -0.38746800 & \\
\hline $\mathrm{O}$ & 0.70540300 & -0.00319300 & 1.70301400 & \\
\hline $\mathrm{C}$ & 0.98073200 & -1.87393100 & -2.70050800 & \\
\hline $\mathrm{H}$ & 2.00806100 & -1.52528800 & -2.55915100 & \\
\hline $\mathrm{H}$ & 1.01470200 & -2.96021000 & -2.81862700 & \\
\hline $\mathrm{H}$ & 0.55700300 & -1.41424900 & -3.59290500 & \\
\hline $\mathrm{C}$ & -3.78807700 & -4.12149600 & 0.00015200 & \\
\hline $\mathrm{H}$ & -4.25203800 & -4.39846200 & 0.95194500 & \\
\hline $\mathrm{H}$ & -4.56366400 & -3.85889900 & -0.71876600 & \\
\hline $\mathrm{H}$ & -3.22178200 & -4.98602400 & -0.35470700 & \\
\hline $\mathrm{C}$ & -3.32993000 & 0.08078400 & 4.13843600 & \\
\hline $\mathrm{H}$ & -3.48161000 & -0.80884200 & 4.75324500 & \\
\hline $\mathrm{H}$ & -2.72428800 & 0.78692500 & 4.71567200 & \\
\hline $\mathrm{H}$ & -4.28587800 & 0.54607400 & 3.89815200 & \\
\hline $\mathrm{C}$ & 1.32315200 & 2.27037200 & 1.48694300 & \\
\hline & & & S93 & \\
\hline
\end{tabular}




$\begin{array}{lrrr}\mathrm{H} & 0.94810900 & 2.78941200 & 2.37545400 \\ \mathrm{H} & 2.32893800 & 1.90587700 & 1.69986800 \\ \mathrm{H} & 1.33766100 & 2.97915600 & 0.65772300 \\ \mathrm{C} & 0.16447100 & -3.56868500 & 3.85709100 \\ \mathrm{C} & -1.22414400 & -3.80074000 & 3.91501800 \\ \mathrm{C} & 0.96160500 & -4.08690300 & 4.90378300 \\ \mathrm{C} & -1.80143500 & -4.46853800 & 4.98869500 \\ \mathrm{H} & -1.85054000 & -3.46139700 & 3.10528200 \\ \mathrm{C} & 0.40322300 & -4.75787500 & 5.98170800 \\ \mathrm{H} & 2.03795600 & -3.98056100 & 4.85919600 \\ \mathrm{C} & -0.98034300 & -4.92943100 & 6.01426900 \\ \mathrm{H} & -2.86891500 & -4.63944400 & 5.03511000 \\ \mathrm{H} & 1.01676100 & -5.15465700 & 6.77967300 \\ \mathrm{C} & 0.76275900 & -2.86989200 & 2.68304100 \\ \mathrm{C} & 0.92848300 & -4.53237300 & 1.31293600 \\ \mathrm{C} & 1.88581700 & -5.20180900 & 2.04042000 \\ \mathrm{H} & 1.17610500 & -3.98830100 & 0.41060900 \\ \mathrm{H} & -0.11668300 & -4.79148000 & 1.42103600 \\ \mathrm{H} & 1.55563200 & -5.77376300 & 2.90334500 \\ \mathrm{C} & 3.31435100 & -5.09622000 & 1.87019200 \\ \mathrm{C} & 3.90495100 & -4.67722900 & 0.65847700 \\ \mathrm{C} & 4.15231300 & -5.37169900 & 2.97197600 \\ \mathrm{C} & 5.28143000 & -4.51713700 & 0.56344600 \\ \mathrm{H} & 3.27851800 & -4.49457300 & -0.20782000 \\ \mathrm{C} & 5.52707400 & -5.18730100 & 2.88057400 \\ \mathrm{H} & 3.70791300 & -5.71169200 & 3.90269500 \\ \mathrm{C} & 6.09418900 & -4.75710900 & 1.67651500 \\ \mathrm{H} & 5.72509500 & -4.19972100 & -0.37456900 \\ \mathrm{H} & 6.15889700 & -5.38161500 & 3.74111100 \\ \mathrm{H} & 7.16835300 & -4.61994300 & 1.60252500 \\ \mathrm{C} & 4.45100300 & -0.71666200 & 3.09821400 \\ \mathrm{H} & 5.37334100 & -0.15218000 & 3.19425200 \\ \mathrm{~N} & -1.58157800 & -5.63368700 & 7.14734300 \\ \mathrm{O} & -0.83383300 & -6.03935000 & 8.03991900 \\ \mathrm{O} & -2.80498800 & -5.78522700 & 7.15263300 \\ & & & \\ & & & \\ & & & \end{array}$




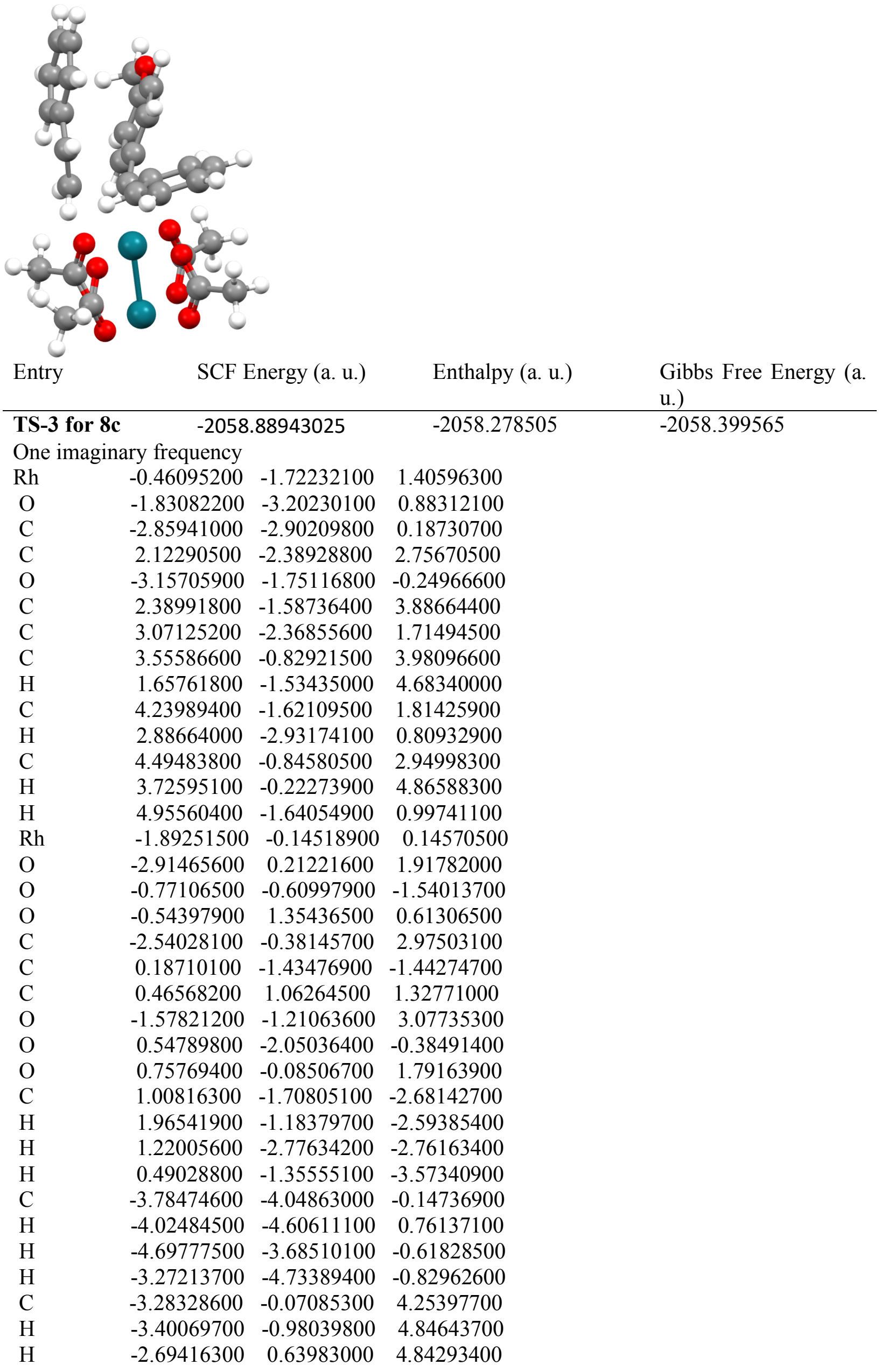




$\begin{array}{lrrr}\mathrm{H} & -4.25534700 & 0.37238800 & 4.03669900 \\ \mathrm{C} & 1.40383000 & 2.18973200 & 1.69454700 \\ \mathrm{H} & 1.00991400 & 2.70097400 & 2.57945700 \\ \mathrm{H} & 2.39374300 & 1.79861100 & 1.93207700 \\ \mathrm{H} & 1.46129600 & 2.91512800 & 0.88133600 \\ \mathrm{C} & 0.19788100 & -3.65063100 & 3.82277600 \\ \mathrm{C} & -1.18254900 & -3.94392300 & 3.86395600 \\ \mathrm{C} & 0.96272900 & -4.03843000 & 4.95337700 \\ \mathrm{C} & -1.78352400 & -4.52394000 & 4.97442400 \\ \mathrm{H} & -1.79092500 & -3.70824700 & 3.00237800 \\ \mathrm{C} & 0.38030500 & -4.61539500 & 6.06586500 \\ \mathrm{H} & 2.03501400 & -3.89008300 & 4.94519100 \\ \mathrm{C} & -1.00274300 & -4.86086300 & 6.08931900 \\ \mathrm{H} & -2.84765500 & -4.72292100 & 4.95741300 \\ \mathrm{H} & 0.97667000 & -4.91510800 & 6.92050400 \\ \mathrm{H} & 5.40769800 & -0.26310600 & 3.02533900 \\ \mathrm{C} & 0.79804000 & -3.04140800 & 2.63301700 \\ \mathrm{C} & 0.86053300 & -4.55361700 & 1.24936600 \\ \mathrm{C} & 1.81326200 & -5.32657600 & 1.89280800 \\ \mathrm{H} & 1.13816800 & -3.97923700 & 0.37519200 \\ \mathrm{H} & -0.18976400 & -4.81148700 & 1.29391100 \\ \mathrm{H} & 2.85967400 & -5.09601200 & 1.71191400 \\ \mathrm{C} & 1.55840200 & -6.28762300 & 2.92907100 \\ \mathrm{C} & 2.63171600 & -6.70690800 & 3.74851400 \\ \mathrm{C} & 0.26049500 & -6.76282100 & 3.22042600 \\ \mathrm{C} & 2.41014800 & -7.53568400 & 4.84014900 \\ \mathrm{H} & 3.63277600 & -6.34800000 & 3.52790400 \\ \mathrm{C} & 0.04245100 & -7.58621400 & 4.31595100 \\ \mathrm{H} & -0.57202500 & -6.47679500 & 2.59013000 \\ \mathrm{C} & 1.11154300 & -7.96553500 & 5.13439400 \\ \mathrm{H} & 3.23982500 & -7.83785500 & 5.47095800 \\ \mathrm{H} & -0.96174500 & -7.92944700 & 4.54181300 \\ \mathrm{H} & 0.93429700 & -8.60304200 & 5.99493000 \\ \mathrm{C} & -2.87285800 & -5.75468900 & 7.27310300 \\ \mathrm{H} & -3.48910100 & -4.85345800 & 7.18367700 \\ \mathrm{H} & -3.14928000 & -6.46367800 & 6.48483000 \\ \mathrm{H} & -3.03696900 & -6.21229200 & 8.24847000 \\ \mathrm{O} & -1.48255700 & -5.43888400 & 7.21714500\end{array}$




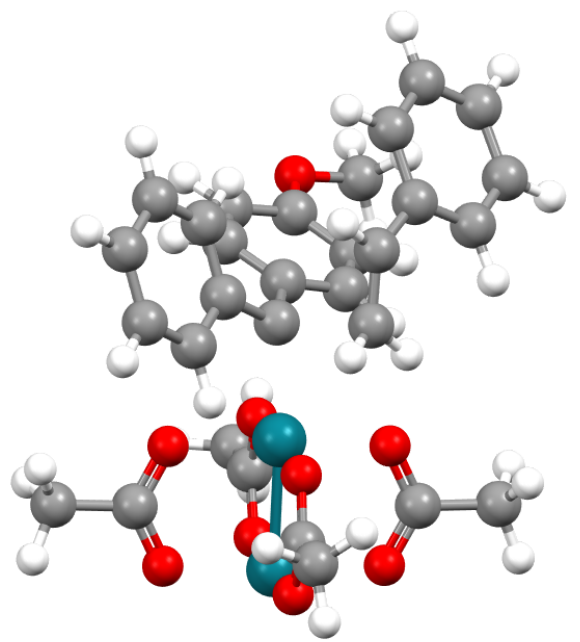

Entry SCF Energy (a. u.) Enthalpy (a. u.) Gibbs Free Energy (a.

\begin{tabular}{|c|c|c|c|c|}
\hline & & & & u.) \\
\hline TS-4 for $8 \mathrm{c}$ & -2058. & .88151746 & -2058.270438 & -2058.393498 \\
\hline One imagina & ary frequency & & & \\
\hline $\mathrm{Rh}$ & -0.49804300 & -1.70286600 & 1.35292400 & \\
\hline $\mathrm{O}$ & -1.85010600 & -3.21622300 & 0.89532000 & \\
\hline $\mathrm{C}$ & -2.89521400 & -2.94746500 & 0.21194400 & \\
\hline $\mathrm{C}$ & 2.05222400 & -2.26030700 & 2.81998600 & \\
\hline $\mathrm{O}$ & -3.20344100 & -1.81724700 & -0.27128600 & \\
\hline $\mathrm{C}$ & 2.44311100 & -1.68014600 & 4.05144200 & \\
\hline $\mathrm{C}$ & 2.87050400 & -1.98038700 & 1.70630800 & \\
\hline $\mathrm{C}$ & 3.59860000 & -0.92289300 & 4.16419400 & \\
\hline $\mathrm{H}$ & 1.81213000 & -1.79181000 & 4.92381100 & \\
\hline $\mathrm{C}$ & 4.05192700 & -1.25936200 & 1.81181600 & \\
\hline $\mathrm{H}$ & 2.55376700 & -2.31603600 & 0.72965000 & \\
\hline $\mathrm{C}$ & 4.43354800 & -0.73231500 & 3.05287000 & \\
\hline $\mathrm{H}$ & 3.88017100 & -0.47434200 & 5.11119200 & \\
\hline $\mathrm{H}$ & 4.65769500 & -1.10678500 & 0.92787400 & \\
\hline $\mathrm{Rh}$ & -1.94588600 & -0.18922000 & 0.03958800 & \\
\hline $\mathrm{O}$ & -2.95403600 & 0.24439100 & 1.79824700 & \\
\hline $\mathrm{O}$ & -0.84156300 & -0.71741400 & -1.63834200 & \\
\hline $\mathrm{O}$ & -0.60147200 & 1.33732200 & 0.42539100 & \\
\hline $\mathrm{C}$ & -2.56244000 & -0.29234800 & 2.87979100 & \\
\hline $\mathrm{C}$ & 0.12140600 & -1.53222000 & -1.51704900 & \\
\hline $\mathrm{C}$ & 0.40980400 & 1.08588500 & 1.15292700 & \\
\hline $\mathrm{O}$ & -1.60114600 & -1.11865000 & 3.00903800 & \\
\hline $\mathrm{O}$ & 0.49843400 & -2.09560200 & -0.43540500 & \\
\hline $\mathrm{O}$ & 0.70397600 & -0.03562200 & 1.67515600 & \\
\hline $\mathrm{C}$ & 0.91877500 & -1.87766200 & -2.75264500 & \\
\hline $\mathrm{H}$ & 0.48512500 & -1.41240900 & -3.63741500 & \\
\hline $\mathrm{H}$ & 1.94886300 & -1.53235600 & -2.62319100 & \\
\hline $\mathrm{H}$ & 0.94858100 & -2.96339100 & -2.87677800 & \\
\hline $\mathrm{C}$ & -3.84869800 & -4.09714500 & -0.01436000 & \\
\hline $\mathrm{H}$ & -4.32112400 & -4.35920100 & 0.93763200 & \\
\hline $\mathrm{H}$ & -4.61799300 & -3.82613600 & -0.73704900 & \\
\hline $\mathrm{H}$ & -3.29661300 & -4.97387000 & -0.36122600 & \\
\hline $\mathrm{C}$ & -3.28071600 & 0.09322500 & 4.15187800 & \\
\hline $\mathrm{H}$ & -3.35799200 & -0.77056000 & 4.81468500 & \\
\hline $\mathrm{H}$ & -2.69724900 & 0.86338200 & 4.66734000 & \\
\hline $\mathrm{H}$ & -4.26955200 & 0.49429600 & 3.92800200 & \\
\hline
\end{tabular}




$\begin{array}{lrrr}\mathrm{C} & 1.34849100 & 2.23009700 & 1.45928900 \\ \mathrm{H} & 1.01695300 & 2.72077200 & 2.38062200 \\ \mathrm{H} & 2.36076100 & 1.85490800 & 1.61709600 \\ \mathrm{H} & 1.33137900 & 2.96481100 & 0.65319100 \\ \mathrm{C} & 0.16326300 & -3.62032800 & 3.85176900 \\ \mathrm{C} & -1.22588300 & -3.83865600 & 3.91027300 \\ \mathrm{C} & 0.94322900 & -4.12029200 & 4.91758900 \\ \mathrm{C} & -1.81185200 & -4.47186700 & 5.00565100 \\ \mathrm{H} & -1.84355800 & -3.51348100 & 3.08685600 \\ \mathrm{C} & 0.36078400 & -4.75570300 & 6.01037700 \\ \mathrm{H} & 2.02247800 & -4.03000400 & 4.87491300 \\ \mathrm{C} & -1.02534500 & -4.92474400 & 6.06537500 \\ \mathrm{H} & -2.88786600 & -4.61786600 & 5.02572900 \\ \mathrm{H} & 0.98966000 & -5.13042700 & 6.81237800 \\ \mathrm{H} & -1.48285500 & -5.41998300 & 6.91654900 \\ \mathrm{C} & 0.78075500 & -2.97170700 & 2.65582700 \\ \mathrm{C} & 0.90468400 & -4.48760700 & 1.32416100 \\ \mathrm{C} & 1.87812800 & -5.19059700 & 2.02197400 \\ \mathrm{H} & 1.14840500 & -3.97285200 & 0.40350000 \\ \mathrm{H} & -0.13168000 & -4.78966200 & 1.40762900 \\ \mathrm{H} & 1.56089200 & -5.76034200 & 2.89045800 \\ \mathrm{C} & 3.29930700 & -5.07276000 & 1.83254800 \\ \mathrm{C} & 3.86906500 & -4.64014200 & 0.61462900 \\ \mathrm{C} & 4.15706900 & -5.33020500 & 2.92503300 \\ \mathrm{C} & 5.24037600 & -4.44835900 & 0.50467400 \\ \mathrm{H} & 3.22924500 & -4.47052300 & -0.24448000 \\ \mathrm{C} & 5.52533900 & -5.11208500 & 2.81915600 \\ \mathrm{H} & 3.72968100 & -5.67679500 & 3.86121000 \\ \mathrm{C} & 6.06990700 & -4.66608100 & 1.60961800 \\ \mathrm{H} & 5.66631800 & -4.11980200 & -0.43780300 \\ \mathrm{H} & 6.17063900 & -5.28939500 & 3.67344300 \\ \mathrm{H} & 7.13952500 & -4.50148100 & 1.52545000 \\ \mathrm{O} & 5.56970700 & -0.01750600 & 3.27130800 \\ \mathrm{C} & 6.45404000 & 0.17846200 & 2.17131200 \\ \mathrm{H} & 7.30225400 & 0.74005200 & 2.56297400 \\ \mathrm{H} & 6.80355200 & -0.77921300 & 1.76821300 \\ \mathrm{H} & 5.97481400 & 0.75394200 & 1.37097100\end{array}$

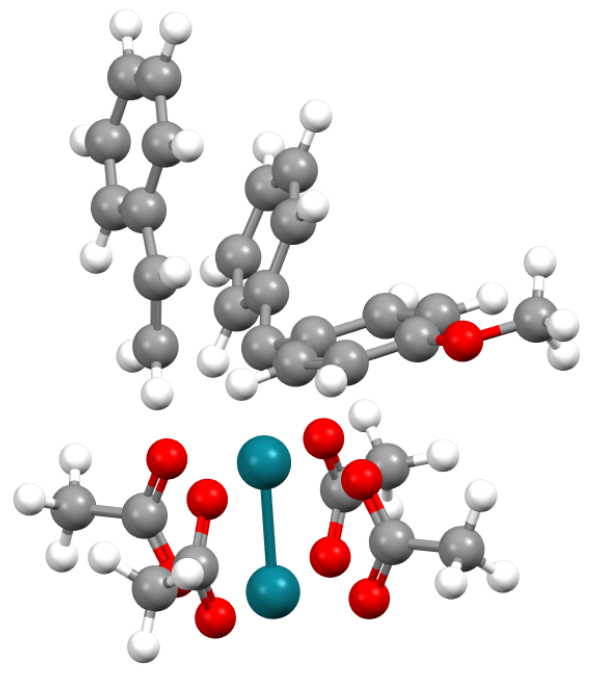


Entry SCF Energy (a. u.) Enthalpy (a. u.) Gibbs Free Energy (a. u.)

\begin{tabular}{|c|c|c|c|}
\hline \multirow{3}{*}{\multicolumn{2}{|c|}{$\begin{array}{ll}\text { TS-5 for 8c } & -2058 \\
\text { One imaginary frequency }\end{array}$}} & .88617062 & -2058.27 \\
\hline & & \multirow[b]{2}{*}{-1.72169300} & \multirow[b]{2}{*}{1.42221300} \\
\hline & & & \\
\hline $\mathrm{O}$ & -1.83913100 & -3.19662000 & 0.89815200 \\
\hline $\mathrm{C}$ & -2.86511200 & -2.89165500 & 0.20014800 \\
\hline $\mathrm{C}$ & 2.09892900 & -2.36651800 & 2.79350100 \\
\hline $\mathrm{O}$ & -3.15568800 & -1.74023600 & -0.23992300 \\
\hline $\mathrm{C}$ & 2.38729300 & -1.60065500 & 3.93854200 \\
\hline $\mathrm{C}$ & 3.02781900 & -2.29464600 & 1.73229800 \\
\hline $\mathrm{C}$ & 3.53820300 & -0.81808600 & 4.04070400 \\
\hline $\mathrm{H}$ & 1.67681800 & -1.57795100 & 4.75607300 \\
\hline $\mathrm{C}$ & 4.18699900 & -1.54605400 & 1.82943500 \\
\hline $\mathrm{H}$ & 2.83064700 & -2.82613200 & 0.81024500 \\
\hline $\mathrm{C}$ & 4.45507800 & -0.79440900 & 2.98631900 \\
\hline $\mathrm{H}$ & 3.70221500 & -0.23842800 & 4.94056300 \\
\hline $\mathrm{H}$ & 4.90140400 & -1.51616600 & 1.01311900 \\
\hline $\mathrm{Rh}$ & -1.88787000 & -0.13672800 & 0.15614800 \\
\hline $\mathrm{O}$ & -2.91242200 & 0.22721700 & 1.92510700 \\
\hline $\mathrm{O}$ & -0.76131100 & -0.60774300 & -1.52556300 \\
\hline $\mathrm{O}$ & -0.53644800 & 1.36048500 & 0.62832300 \\
\hline $\mathrm{C}$ & -2.53926700 & -0.36225500 & 2.98470600 \\
\hline $\mathrm{C}$ & 0.18811800 & -1.44150600 & -1.42635700 \\
\hline $\mathrm{C}$ & 0.47633300 & 1.06150400 & 1.33558400 \\
\hline $\mathrm{O}$ & -1.58185200 & -1.19692500 & 3.09023400 \\
\hline $\mathrm{O}$ & 0.54297000 & -2.05825600 & -0.36648500 \\
\hline $\mathrm{O}$ & 0.75829300 & -0.08595500 & 1.80529700 \\
\hline $\mathrm{C}$ & 0.99830100 & -1.74229300 & -2.66580200 \\
\hline $\mathrm{H}$ & 2.02829100 & -1.40699200 & -2.51146600 \\
\hline $\mathrm{H}$ & 1.02519300 & -2.82221700 & -2.83297600 \\
\hline $\mathrm{H}$ & 0.57693900 & -1.23972000 & -3.53603800 \\
\hline $\mathrm{C}$ & -3.79726100 & -4.03350100 & -0.13169000 \\
\hline $\mathrm{H}$ & -4.05310700 & -4.57715800 & 0.78120500 \\
\hline $\mathrm{H}$ & -4.70153300 & -3.66689600 & -0.61687400 \\
\hline $\mathrm{H}$ & -3.28375100 & -4.73231200 & -0.79925100 \\
\hline $\mathrm{C}$ & -3.27757600 & -0.04066800 & 4.26363900 \\
\hline $\mathrm{H}$ & -3.41787800 & -0.95040200 & 4.85092600 \\
\hline $\mathrm{H}$ & -2.67270100 & 0.65197500 & 4.85802500 \\
\hline $\mathrm{H}$ & -4.23853900 & 0.42617700 & 4.04674900 \\
\hline $\mathrm{C}$ & 1.45907800 & 2.17030700 & 1.63664500 \\
\hline $\mathrm{H}$ & 0.92379600 & 3.05829000 & 1.98102000 \\
\hline $\mathrm{H}$ & 2.18296600 & 1.84862500 & 2.38567800 \\
\hline $\mathrm{H}$ & 1.98752500 & 2.43700800 & 0.71589500 \\
\hline $\mathrm{C}$ & 0.17974100 & -3.65310700 & 3.84154800 \\
\hline $\mathrm{C}$ & -1.20287300 & -3.93961100 & 3.87923300 \\
\hline $\mathrm{C}$ & 0.95829300 & -4.06697800 & 4.94724900 \\
\hline $\mathrm{C}$ & -1.78329700 & -4.54134700 & 4.99138800 \\
\hline $\mathrm{H}$ & -1.81319400 & -3.68052700 & 3.02618100 \\
\hline $\mathrm{C}$ & 0.37746400 & -4.66747200 & 6.05933100 \\
\hline $\mathrm{H}$ & 2.03101200 & -3.92410100 & 4.92406200 \\
\hline $\mathrm{C}$ & -0.99912100 & -4.89759300 & 6.09106200 \\
\hline $\mathrm{H}$ & -2.85074800 & -4.74054800 & 4.99739800 \\
\hline
\end{tabular}




$\begin{array}{lrrr}\mathrm{H} & 1.00229300 & -4.97560100 & 6.89149900 \\ \mathrm{C} & 0.77759600 & -3.00961600 & 2.65731200 \\ \mathrm{C} & 0.85801600 & -4.56832600 & 1.25967300 \\ \mathrm{C} & 1.81426400 & -5.32301700 & 1.90963700 \\ \mathrm{H} & 1.13224600 & -3.97997500 & 0.39402500 \\ \mathrm{H} & -0.19251700 & -4.82377600 & 1.31301400 \\ \mathrm{H} & 2.85826200 & -5.07556000 & 1.73433100 \\ \mathrm{C} & 1.57199200 & -6.30435800 & 2.93246600 \\ \mathrm{C} & 2.65347400 & -6.73065700 & 3.73644000 \\ \mathrm{C} & 0.28239200 & -6.80711100 & 3.21298300 \\ \mathrm{C} & 2.44856300 & -7.59934000 & 4.80057900 \\ \mathrm{H} & 3.64838900 & -6.35008600 & 3.52440000 \\ \mathrm{C} & 0.08132200 & -7.67110100 & 4.27996900 \\ \mathrm{H} & -0.55711700 & -6.51081300 & 2.59657200 \\ \mathrm{C} & 1.15920300 & -8.06360100 & 5.08077000 \\ \mathrm{H} & 3.28539500 & -7.90934100 & 5.41809300 \\ \mathrm{H} & -0.91673300 & -8.03717400 & 4.49644100 \\ \mathrm{H} & 0.99566600 & -8.73628600 & 5.91693300 \\ \mathrm{H} & -1.45401000 & -5.36932800 & 6.95688000 \\ \mathrm{O} & 5.61640000 & -0.08267000 & 2.98011100 \\ \mathrm{C} & 5.90817300 & 0.72115300 & 4.11884500 \\ \mathrm{H} & 5.13644300 & 1.48380800 & 4.27629500 \\ \mathrm{H} & 6.00227000 & 0.11184300 & 5.02524900 \\ \mathrm{H} & 6.86099700 & 1.20758900 & 3.90914500\end{array}$

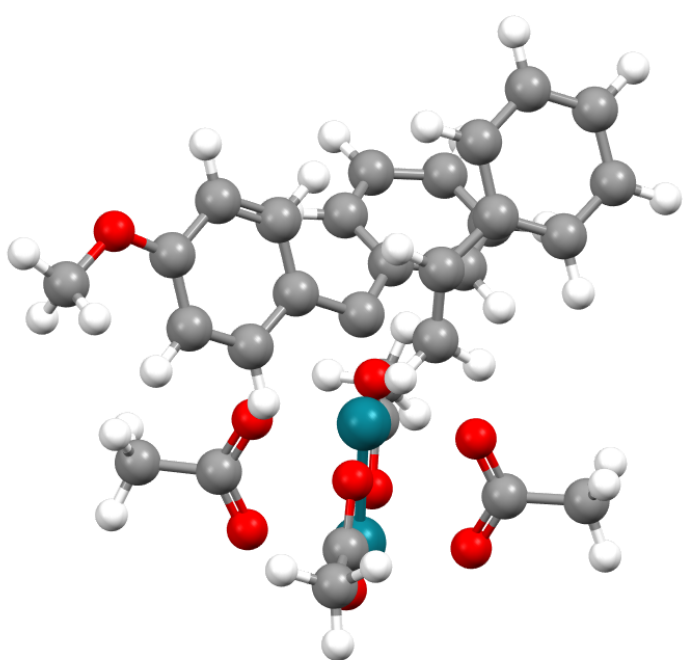

Entry

SCF Energy (a. u.)

Enthalpy (a. u.)

Gibbs Free Energy (a. u.)

\begin{tabular}{lrrc}
\hline \multicolumn{2}{l}{ TS-6 for 8c } & -2058.88208297 & -2058.270 \\
One imaginary frequency & & \\
Rh & -0.49198900 & -1.69879000 & 1.34835000 \\
O & -1.80521500 & -3.23950100 & 0.86307000 \\
C & -2.84881600 & -2.98871500 & 0.17045200 \\
C & 2.06805400 & -2.21321500 & 2.80240100 \\
O & -3.17905100 & -1.86083500 & -0.30279000 \\
C & 2.37940300 & -1.55509400 & 4.01273900 \\
C & 2.91857700 & -1.97404200 & 1.70289100 \\
C & 3.50168400 & -0.73604600 & 4.12204900 \\
H & 1.71546100 & -1.65214700 & 4.86258900 \\
C & 4.05569100 & -1.18536100 & 1.82175100
\end{tabular}




\begin{tabular}{|c|c|c|c|}
\hline $\mathrm{H}$ & 2.65571300 & -2.38235300 & 0.73854900 \\
\hline $\mathrm{C}$ & 4.35996700 & -0.56192300 & 3.03534800 \\
\hline $\mathrm{H}$ & 3.70547100 & -0.23525100 & 5.06400800 \\
\hline $\mathrm{H}$ & 4.69926700 & -1.04358300 & 0.95861100 \\
\hline $\mathrm{Rh}$ & -1.96866000 & -0.20500100 & 0.04404400 \\
\hline $\mathrm{O}$ & -3.00526400 & 0.17435800 & 1.79999000 \\
\hline $\mathrm{O}$ & -0.83219600 & -0.67980100 & -1.62869000 \\
\hline $\mathrm{O}$ & -0.67087000 & 1.35117500 & 0.46868000 \\
\hline $\mathrm{C}$ & -2.61069700 & -0.37063200 & 2.87624800 \\
\hline $\mathrm{C}$ & 0.14925200 & -1.47327400 & -1.50957900 \\
\hline $\mathrm{C}$ & 0.34104100 & 1.11438900 & 1.20052600 \\
\hline $\mathrm{O}$ & -1.62640000 & -1.16967800 & 3.00234200 \\
\hline $\mathrm{O}$ & 0.52723900 & -2.04730200 & -0.43443200 \\
\hline $\mathrm{O}$ & 0.66605200 & -0.00939300 & 1.69836400 \\
\hline $\mathrm{C}$ & 0.97694300 & -1.76156400 & -2.73999700 \\
\hline $\mathrm{H}$ & 1.19087500 & -2.83081800 & -2.80268100 \\
\hline $\mathrm{H}$ & 0.46257500 & -1.42446800 & -3.63980900 \\
\hline $\mathrm{H}$ & 1.93316200 & -1.23524300 & -2.65477700 \\
\hline $\mathrm{C}$ & -3.76974100 & -4.15922800 & -0.08217800 \\
\hline $\mathrm{H}$ & -4.54407000 & -3.89476600 & -0.80188300 \\
\hline $\mathrm{H}$ & -3.19245300 & -5.01279500 & -0.44561100 \\
\hline $\mathrm{H}$ & -4.23703800 & -4.45442900 & 0.86256800 \\
\hline $\mathrm{C}$ & -3.35565600 & -0.03154200 & 4.14635300 \\
\hline $\mathrm{H}$ & -4.35654900 & 0.33579000 & 3.91808600 \\
\hline $\mathrm{H}$ & -3.40705100 & -0.90778800 & 4.79520800 \\
\hline $\mathrm{H}$ & -2.80702400 & 0.75204700 & 4.67951400 \\
\hline $\mathrm{C}$ & 1.23664600 & 2.28289100 & 1.54243900 \\
\hline $\mathrm{H}$ & 0.80765600 & 2.81620900 & 2.39745200 \\
\hline $\mathrm{H}$ & 2.23267500 & 1.93271000 & 1.81550400 \\
\hline $\mathrm{H}$ & 1.28997600 & 2.97751100 & 0.70240800 \\
\hline $\mathrm{C}$ & 0.19595500 & -3.59817200 & 3.83050800 \\
\hline $\mathrm{C}$ & -1.18127800 & -3.87965700 & 3.87356600 \\
\hline $\mathrm{C}$ & 0.97102400 & -4.04669500 & 4.92796400 \\
\hline $\mathrm{C}$ & -1.77504800 & -4.51192100 & 4.96354900 \\
\hline $\mathrm{H}$ & -1.79985600 & -3.60066600 & 3.03341800 \\
\hline $\mathrm{C}$ & 0.39974200 & -4.68052800 & 6.01691700 \\
\hline $\mathrm{H}$ & 2.04636800 & -3.91205500 & 4.91040800 \\
\hline $\mathrm{C}$ & -0.98740400 & -4.90697700 & 6.05201900 \\
\hline $\mathrm{H}$ & -2.84220200 & -4.69533200 & 4.95120400 \\
\hline $\mathrm{H}$ & 1.00537700 & -5.02279300 & 6.84948000 \\
\hline $\mathrm{H}$ & 5.24322000 & 0.06283500 & 3.12682000 \\
\hline $\mathrm{C}$ & 0.80349100 & -2.95418600 & 2.64246300 \\
\hline $\mathrm{C}$ & 0.96245300 & -4.45623200 & 1.29327900 \\
\hline $\mathrm{C}$ & 1.91584500 & -5.15671800 & 2.01837200 \\
\hline $\mathrm{H}$ & 1.22581300 & -3.94444400 & 0.37629400 \\
\hline $\mathrm{H}$ & -0.07648400 & -4.75288600 & 1.35741100 \\
\hline $\mathrm{H}$ & 1.57112600 & -5.70976200 & 2.88744100 \\
\hline $\mathrm{C}$ & 3.34364100 & -5.09914700 & 1.84785500 \\
\hline $\mathrm{C}$ & 3.95156800 & -4.69179100 & 0.64001800 \\
\hline $\mathrm{C}$ & 4.17153200 & -5.42602400 & 2.94434600 \\
\hline $\mathrm{C}$ & 5.33393200 & -4.59541800 & 0.54338200 \\
\hline $\mathrm{H}$ & 3.33331600 & -4.47154200 & -0.22337000 \\
\hline $\mathrm{C}$ & 5.55318700 & -5.30956300 & 2.85017800 \\
\hline
\end{tabular}




$\begin{array}{lrrr}\mathrm{H} & 3.71211500 & -5.75197200 & 3.87282300 \\ \mathrm{C} & 6.13745500 & -4.89178400 & 1.64972800 \\ \mathrm{H} & 5.79004000 & -4.28689600 & -0.39170000 \\ \mathrm{H} & 6.17729100 & -5.54572300 & 3.70594700 \\ \mathrm{H} & 7.21687200 & -4.80675700 & 1.57343700 \\ \mathrm{O} & -1.45920100 & -5.52475600 & 7.16407200 \\ \mathrm{C} & -2.85960200 & -5.78392500 & 7.24542700 \\ \mathrm{H} & -3.01979900 & -6.26481100 & 8.21028100 \\ \mathrm{H} & -3.43814500 & -4.85468700 & 7.19906700 \\ \mathrm{H} & -3.18698500 & -6.45529100 & 6.44378600\end{array}$




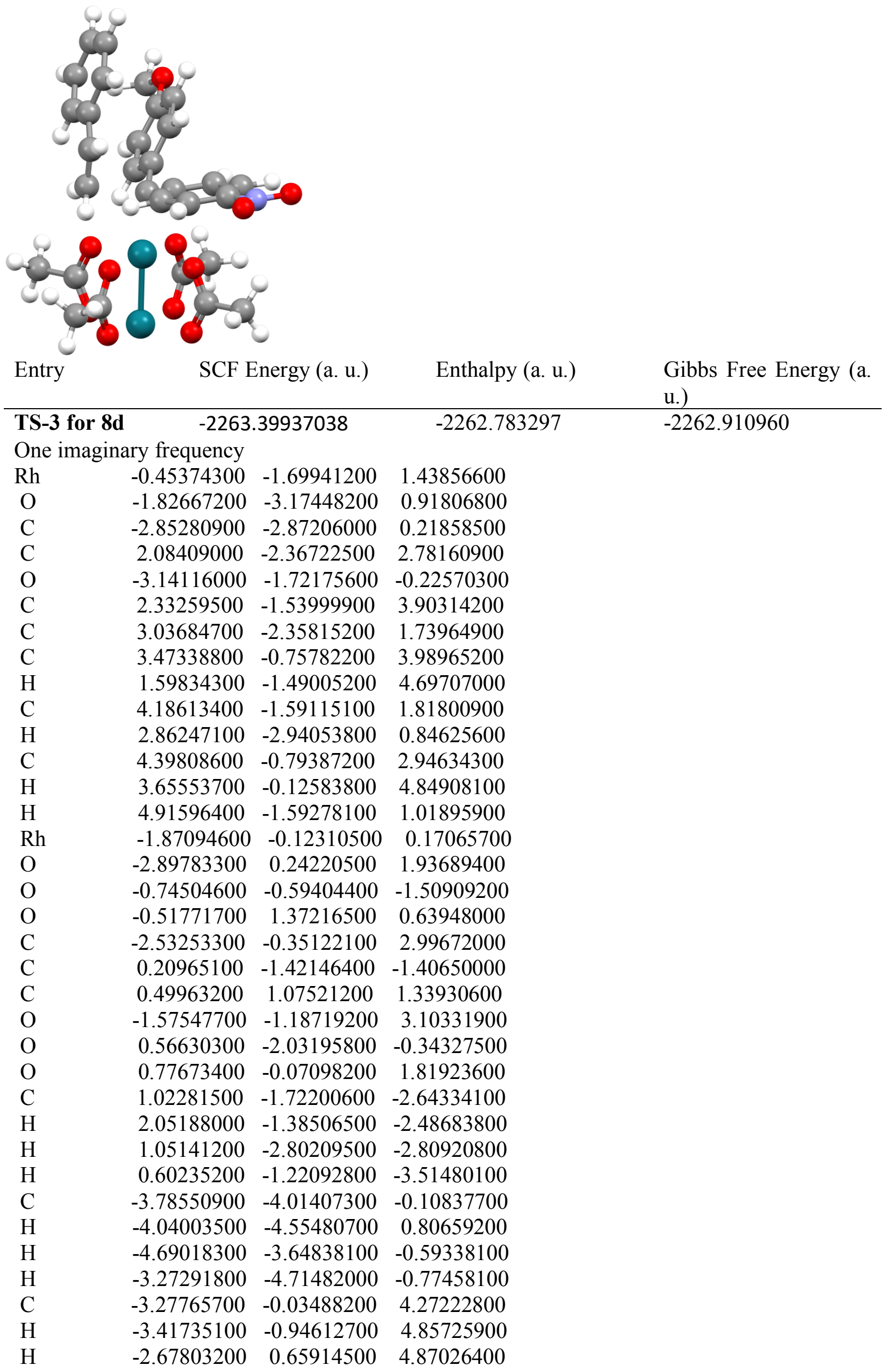




$\begin{array}{lrrr}\mathrm{H} & -4.23912200 & 0.42889200 & 4.05123600 \\ \mathrm{C} & 1.50591600 & 2.16948100 & 1.60788900 \\ \mathrm{H} & 1.00686600 & 3.13702500 & 1.67973500 \\ \mathrm{H} & 2.06689000 & 1.95567000 & 2.51845100 \\ \mathrm{H} & 2.21046300 & 2.20884900 & 0.77018500 \\ \mathrm{C} & 0.18437700 & -3.65327100 & 3.83139700 \\ \mathrm{C} & -1.19885700 & -3.94419400 & 3.88521500 \\ \mathrm{C} & 0.97035300 & -4.07129300 & 4.93980900 \\ \mathrm{C} & -1.77965000 & -4.55444200 & 4.98707900 \\ \mathrm{H} & -1.81803800 & -3.68226700 & 3.03919300 \\ \mathrm{C} & 0.40546900 & -4.67556500 & 6.04415800 \\ \mathrm{H} & 2.04208900 & -3.91827800 & 4.91967600 \\ \mathrm{C} & -0.97866000 & -4.92251600 & 6.08008900 \\ \mathrm{H} & -2.84378900 & -4.75299600 & 4.98432200 \\ \mathrm{H} & 1.01298300 & -4.99714700 & 6.88238700 \\ \mathrm{C} & 0.76215600 & -3.00366700 & 2.66993200 \\ \mathrm{C} & 0.85987500 & -4.60495800 & 1.20755700 \\ \mathrm{C} & 1.80925500 & -5.35701700 & 1.85681500 \\ \mathrm{H} & 1.12903800 & -3.99705000 & 0.35380600 \\ \mathrm{H} & -0.19586000 & -4.82826400 & 1.28700900 \\ \mathrm{H} & 2.85692100 & -5.14138500 & 1.66234000 \\ \mathrm{C} & 1.55306200 & -6.30897300 & 2.90780700 \\ \mathrm{C} & 2.62399900 & -6.71749000 & 3.73378000 \\ \mathrm{C} & 0.25681800 & -6.78718600 & 3.19650500 \\ \mathrm{C} & 2.40134400 & -7.53928700 & 4.83122600 \\ \mathrm{H} & 3.62483600 & -6.35718300 & 3.51435900 \\ \mathrm{C} & 0.03741500 & -7.60546200 & 4.29583100 \\ \mathrm{H} & -0.57440500 & -6.51007200 & 2.56007100 \\ \mathrm{C} & 1.10412500 & -7.97385300 & 5.12241100 \\ \mathrm{H} & 3.22989300 & -7.83297300 & 5.46746400 \\ \mathrm{H} & -0.96604000 & -7.95324700 & 4.51820400 \\ \mathrm{H} & 0.92588800 & -8.60684900 & 5.98601400 \\ \mathrm{C} & -2.82987500 & -5.84807000 & 7.27073900 \\ \mathrm{H} & -3.44543700 & -4.94406700 & 7.21682300 \\ \mathrm{H} & -3.12024800 & -6.53686700 & 6.47024300 \\ \mathrm{H} & -2.97302900 & -6.33007800 & 8.23729900 \\ \mathrm{O} & -1.43904800 & -5.53059000 & 7.19502900 \\ \mathrm{~N} & 5.59674600 & 0.01986500 & 3.02933800 \\ \mathrm{O} & 6.40566300 & -0.03182200 & 2.09561200 \\ \mathrm{O} & 5.75467700 & 0.73018700 & 4.02901600\end{array}$




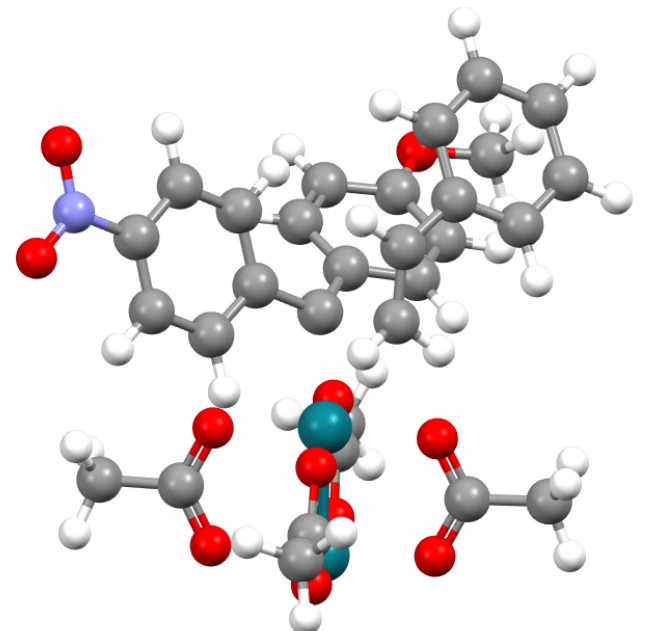

Entry SCF Energy (a. u.) Enthalpy (a. u.) Gibbs Free Energy (a.

\begin{tabular}{|c|c|c|c|c|}
\hline & & & & u.) \\
\hline TS-4 for 8d & -2263 & 38755921 & -2262.771413 & -2262.901400 \\
\hline One imagina & ary frequency & & & \\
\hline $\mathrm{Rh}$ & -0.50522700 & -1.69931400 & 1.35024600 & \\
\hline $\mathrm{O}$ & -1.85518100 & -3.21886700 & 0.90954600 & \\
\hline $\mathrm{C}$ & -2.90390200 & -2.95599900 & 0.22778500 & \\
\hline $\mathrm{C}$ & 2.04742300 & -2.24832200 & 2.82295900 & \\
\hline $\mathrm{O}$ & -3.21339300 & -1.82960600 & -0.26215100 & \\
\hline $\mathrm{C}$ & 2.46046500 & -1.70187700 & 4.06391100 & \\
\hline $\mathrm{C}$ & 2.85871500 & -1.95708900 & 1.70556400 & \\
\hline $\mathrm{C}$ & 3.63098500 & -0.97070100 & 4.18129300 & \\
\hline $\mathrm{H}$ & 1.83497600 & -1.81480000 & 4.94023300 & \\
\hline $\mathrm{C}$ & 4.05503200 & -1.26361400 & 1.81581800 & \\
\hline $\mathrm{H}$ & 2.52513200 & -2.26692100 & 0.72568800 & \\
\hline $\mathrm{C}$ & 4.46082500 & -0.77606400 & 3.06568900 & \\
\hline $\mathrm{H}$ & 3.93018100 & -0.54718100 & 5.13418300 & \\
\hline $\mathrm{H}$ & 4.65607600 & -1.10340500 & 0.93024600 & \\
\hline $\mathrm{Rh}$ & -1.95809300 & -0.19732000 & 0.03369500 & \\
\hline $\mathrm{O}$ & -2.96096900 & 0.24493300 & 1.79307600 & \\
\hline $\mathrm{O}$ & -0.85762300 & -0.73552400 & -1.64313900 & \\
\hline $\mathrm{O}$ & -0.61396400 & 1.33236700 & 0.40611600 & \\
\hline $\mathrm{C}$ & -2.56923100 & -0.28730100 & 2.87605300 & \\
\hline $\mathrm{C}$ & 0.10690100 & -1.54768200 & -1.52007400 & \\
\hline $\mathrm{C}$ & 0.39980500 & 1.08722500 & 1.13157100 & \\
\hline $\mathrm{O}$ & -1.60596800 & -1.11269800 & 3.00682900 & \\
\hline $\mathrm{O}$ & 0.48782300 & -2.10331100 & -0.43492700 & \\
\hline $\mathrm{O}$ & 0.69611300 & -0.03121900 & 1.66075100 & \\
\hline $\mathrm{C}$ & 0.90133300 & -1.90051400 & -2.75496000 & \\
\hline $\mathrm{H}$ & 0.46492700 & -1.44122500 & -3.64144000 & \\
\hline $\mathrm{H}$ & 1.93143600 & -1.55357800 & -2.63022100 & \\
\hline $\mathrm{H}$ & 0.93149700 & -2.98699700 & -2.87210000 & \\
\hline $\mathrm{C}$ & -3.85699300 & -4.10783700 & 0.01422600 & \\
\hline $\mathrm{H}$ & -4.32399300 & -4.36396400 & 0.97049300 & \\
\hline $\mathrm{H}$ & -4.63030100 & -3.84218500 & -0.70609100 & \\
\hline $\mathrm{H}$ & -3.30575700 & -4.98621600 & -0.32975500 & \\
\hline $\mathrm{C}$ & -3.28712700 & 0.09877700 & 4.14765500 & \\
\hline $\mathrm{H}$ & -3.39287400 & -0.77290300 & 4.79631300 & \\
\hline $\mathrm{H}$ & -2.68662400 & 0.84360400 & 4.68029400 & \\
\hline $\mathrm{H}$ & -4.26316000 & 0.52896800 & 3.92244800 & \\
\hline
\end{tabular}




$\begin{array}{lrrr}\mathrm{C} & 1.33978100 & 2.23276200 & 1.42700600 \\ \mathrm{H} & 1.02713900 & 2.71323900 & 2.36020300 \\ \mathrm{H} & 2.35667400 & 1.86006800 & 1.55966900 \\ \mathrm{H} & 1.30253000 & 2.97405400 & 0.62781500 \\ \mathrm{C} & 0.15706800 & -3.60908200 & 3.84232000 \\ \mathrm{C} & -1.23450200 & -3.81788000 & 3.90129700 \\ \mathrm{C} & 0.94162400 & -4.12709200 & 4.89746000 \\ \mathrm{C} & -1.82589000 & -4.45830200 & 4.98404700 \\ \mathrm{H} & -1.85387400 & -3.48112800 & 3.08488100 \\ \mathrm{C} & 0.37011100 & -4.77131100 & 5.98546100 \\ \mathrm{H} & 2.02009800 & -4.04316900 & 4.85291000 \\ \mathrm{C} & -1.01611600 & -4.91725700 & 6.01977700 \\ \mathrm{H} & -2.89642700 & -4.60951000 & 5.03004300 \\ \mathrm{H} & 0.97618000 & -5.16758500 & 6.78944600 \\ \mathrm{C} & 0.77225600 & -2.94327100 & 2.64923900 \\ \mathrm{C} & 0.90441200 & -4.50273700 & 1.32627600 \\ \mathrm{C} & 1.88273800 & -5.19573200 & 2.02525000 \\ \mathrm{H} & 1.14384400 & -3.97739100 & 0.41046500 \\ \mathrm{H} & -0.13145600 & -4.80645500 & 1.41325200 \\ \mathrm{H} & 1.57089300 & -5.78099600 & 2.88559200 \\ \mathrm{C} & 3.30142300 & -5.04770400 & 1.84279700 \\ \mathrm{C} & 3.86612500 & -4.60348200 & 0.62624900 \\ \mathrm{C} & 4.15920500 & -5.27935500 & 2.94146600 \\ \mathrm{C} & 5.23211700 & -4.37552300 & 0.52368500 \\ \mathrm{H} & 3.22698100 & -4.45457000 & -0.23709700 \\ \mathrm{C} & 5.52048400 & -5.01952200 & 2.84408400 \\ \mathrm{H} & 3.73731900 & -5.63874700 & 3.87527000 \\ \mathrm{C} & 6.05935000 & -4.56223100 & 1.63601300 \\ \mathrm{H} & 5.65503600 & -4.03965500 & -0.41744900 \\ \mathrm{H} & 6.16514500 & -5.17417600 & 3.70309500 \\ \mathrm{H} & 7.12416100 & -4.36641600 & 1.55842400 \\ \mathrm{O} & 5.61437200 & -0.09609700 & 3.28926300 \\ \mathrm{C} & 6.50439500 & 0.08716000 & 2.19035300 \\ \mathrm{H} & 7.37222400 & 0.61109300 & 2.59066300 \\ \mathrm{H} & 6.81762800 & -0.87616100 & 1.77164100 \\ \mathrm{H} & 6.04482900 & 0.69326900 & 1.40152200 \\ \mathrm{~N} & -1.63132200 & -5.59103500 & 7.16209200 \\ \mathrm{O} & -0.89328800 & -5.99390900 & 8.06453700 \\ \mathrm{O} & -2.85746100 & -5.72254100 & 7.16734700\end{array}$

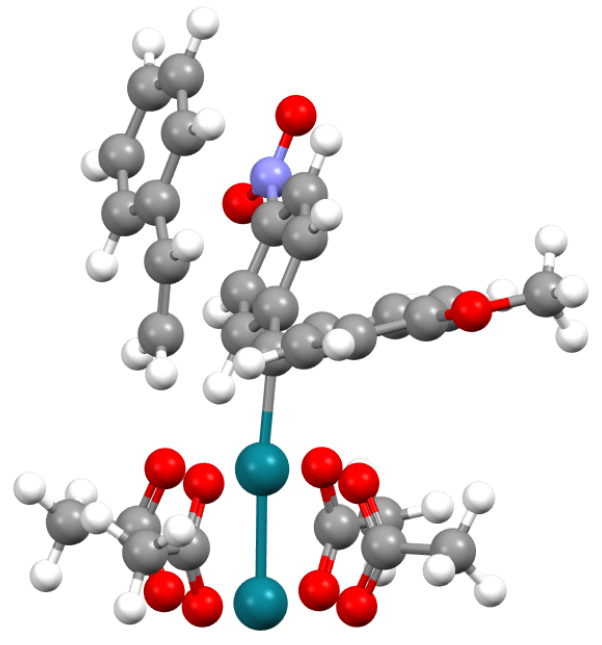


Entry SCF Energy (a. u.) Enthalpy (a. u.) Gibbs Free Energy (a. u.)

\begin{tabular}{|c|c|c|c|c|}
\hline & & & & \\
\hline TS-5 for $8 d$ & -2263 & 39283928 & -2262.776802 & -2262.904586 \\
\hline One imagin & ary frequency & & & \\
\hline $\mathrm{Rh}$ & -0.46253200 & -1.69438700 & 1.42364300 & \\
\hline $\mathrm{O}$ & -1.82404500 & -3.18900200 & 0.92953700 & \\
\hline $\mathrm{C}$ & -2.85891000 & -2.90310600 & 0.23440600 & \\
\hline $\mathrm{C}$ & 2.10006700 & -2.29109400 & 2.80774900 & \\
\hline $\mathrm{O}$ & -3.15294100 & -1.76431100 & -0.23323300 & \\
\hline $\mathrm{C}$ & 2.41607200 & -1.56029000 & 3.96933800 & \\
\hline $\mathrm{C}$ & 3.00960000 & -2.20401600 & 1.73021200 & \\
\hline $\mathrm{C}$ & 3.57499600 & -0.79055800 & 4.06825900 & \\
\hline $\mathrm{H}$ & 1.72087600 & -1.54859200 & 4.80048700 & \\
\hline $\mathrm{C}$ & 4.18002900 & -1.47585400 & 1.82779200 & \\
\hline $\mathrm{H}$ & 2.78492100 & -2.70892400 & 0.79985100 & \\
\hline $\mathrm{C}$ & 4.47470800 & -0.75416100 & 2.99868200 & \\
\hline $\mathrm{H}$ & 3.76021600 & -0.23197900 & 4.97704000 & \\
\hline $\mathrm{H}$ & 4.88247000 & -1.43433400 & 1.00188400 & \\
\hline $\mathrm{Rh}$ & -1.89909000 & -0.14386000 & 0.13433400 & \\
\hline $\mathrm{O}$ & -2.92661600 & 0.23710600 & 1.89726800 & \\
\hline $\mathrm{O}$ & -0.76769900 & -0.63295500 & -1.53830000 & \\
\hline $\mathrm{O}$ & -0.56024200 & 1.37110900 & 0.58185600 & \\
\hline $\mathrm{C}$ & -2.55233300 & -0.33466800 & 2.96497700 & \\
\hline $\mathrm{C}$ & 0.18651600 & -1.45888200 & -1.42718800 & \\
\hline $\mathrm{C}$ & 0.45722200 & 1.09337600 & 1.28952100 & \\
\hline $\mathrm{O}$ & -1.58633500 & -1.16003600 & 3.08296000 & \\
\hline $\mathrm{O}$ & 0.54655300 & -2.05539500 & -0.35643000 & \\
\hline $\mathrm{O}$ & 0.74856600 & -0.04399100 & 1.78041200 & \\
\hline $\mathrm{C}$ & 0.99635500 & -1.77830100 & -2.66153100 & \\
\hline $\mathrm{H}$ & 2.02811400 & -1.44755400 & -2.50981300 & \\
\hline $\mathrm{H}$ & 1.01665700 & -2.86032200 & -2.81590100 & \\
\hline $\mathrm{H}$ & 0.57819900 & -1.28377100 & -3.53782600 & \\
\hline $\mathrm{C}$ & -3.80978600 & -4.04688000 & -0.02740400 & \\
\hline $\mathrm{H}$ & -4.27162000 & -4.34973300 & 0.91747900 & \\
\hline $\mathrm{H}$ & -4.58590300 & -3.75124300 & -0.73280000 & \\
\hline $\mathrm{H}$ & -3.25626900 & -4.90605400 & -0.41420700 & \\
\hline $\mathrm{C}$ & -3.29776000 & -0.00838900 & 4.23786300 & \\
\hline $\mathrm{H}$ & -3.47583100 & -0.92268200 & 4.80803900 & \\
\hline $\mathrm{H}$ & -2.67897300 & 0.65309000 & 4.85272800 & \\
\hline $\mathrm{H}$ & -4.24054900 & 0.49107500 & 4.01489800 & \\
\hline $\mathrm{C}$ & 1.43821000 & 2.21099000 & 1.55926200 & \\
\hline $\mathrm{H}$ & 0.90111800 & 3.12827800 & 1.80950100 & \\
\hline $\mathrm{H}$ & 2.12129500 & 1.93671200 & 2.36340100 & \\
\hline $\mathrm{H}$ & 2.01710000 & 2.39965400 & 0.64928800 & \\
\hline $\mathrm{C}$ & 0.19233500 & -3.59112000 & 3.84674800 & \\
\hline $\mathrm{C}$ & -1.18690600 & -3.89336200 & 3.88610300 & \\
\hline $\mathrm{C}$ & 0.99471000 & -4.04443200 & 4.91918200 & \\
\hline $\mathrm{C}$ & -1.74533900 & -4.57779000 & 4.95663100 & \\
\hline $\mathrm{H}$ & -1.81376600 & -3.59940900 & 3.05793500 & \\
\hline $\mathrm{C}$ & 0.45183300 & -4.72726800 & 5.99801200 & \\
\hline $\mathrm{H}$ & 2.06337300 & -3.88170000 & 4.89270100 & \\
\hline $\mathrm{C}$ & -0.91587400 & -4.98551300 & 5.99973100 & \\
\hline $\mathrm{H}$ & -2.80021600 & -4.81665900 & 4.98290000 & \\
\hline
\end{tabular}




$\begin{array}{lrrr}\mathrm{H} & 1.07261000 & -5.09070100 & 6.80517200 \\ \mathrm{C} & 0.77433300 & -2.91049400 & 2.67054700 \\ \mathrm{C} & 0.88087300 & -4.57713100 & 1.27406500 \\ \mathrm{C} & 1.81139500 & -5.32464700 & 1.95585000 \\ \mathrm{H} & 1.17727300 & -3.97716200 & 0.42422900 \\ \mathrm{H} & -0.17678300 & -4.80267200 & 1.32219900 \\ \mathrm{H} & 2.86170200 & -5.08412700 & 1.80776600 \\ \mathrm{C} & 1.53253300 & -6.30702500 & 2.97373500 \\ \mathrm{C} & 2.58135200 & -6.72308400 & 3.82452400 \\ \mathrm{C} & 0.23621000 & -6.81947300 & 3.20074400 \\ \mathrm{C} & 2.33559800 & -7.58165800 & 4.88911100 \\ \mathrm{H} & 3.58230200 & -6.33865400 & 3.65213700 \\ \mathrm{C} & -0.00501700 & -7.67662400 & 4.26548200 \\ \mathrm{H} & -0.57789100 & -6.53672900 & 2.54434800 \\ \mathrm{C} & 1.03857600 & -8.05044700 & 5.11994800 \\ \mathrm{H} & 3.14690700 & -7.87849500 & 5.54566500 \\ \mathrm{H} & -1.00870300 & -8.04992900 & 4.44077800 \\ \mathrm{H} & 0.84097000 & -8.70990300 & 5.95893200 \\ \mathrm{O} & 5.64116500 & -0.05680200 & 2.98918000 \\ \mathrm{C} & 5.96663800 & 0.71531400 & 4.14232400 \\ \mathrm{H} & 5.20882000 & 1.48405500 & 4.33207500 \\ \mathrm{H} & 6.06942200 & 0.08155500 & 5.03045400 \\ \mathrm{H} & 6.92191200 & 1.19293400 & 3.92475800 \\ \mathrm{~N} & -1.48822000 & -5.75576300 & 7.10379800 \\ \mathrm{O} & -0.72922400 & -6.14600100 & 7.99383700 \\ \mathrm{O} & -2.69980300 & -5.98379300 & 7.08498300\end{array}$




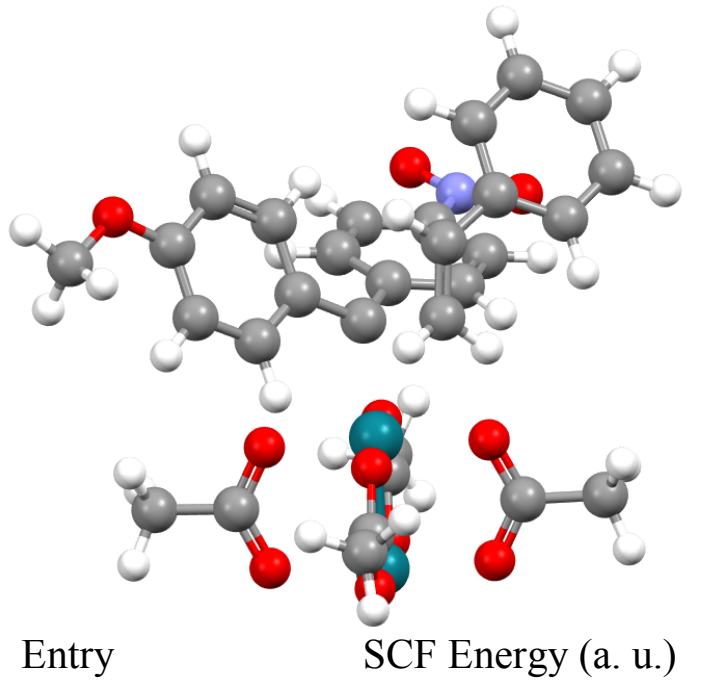

TS-6 for 8d

One imaginary frequency

$\mathrm{Rh}$

$\mathrm{O}$

C

C

$\mathrm{O}$

C

C

C

$\mathrm{H}$

C

$\mathrm{H}$

C

$\mathrm{H}$

$\mathrm{H}$

$\mathrm{Rh}$

$\mathrm{O}$

O

$\mathrm{O}$

C

C

C

$\mathrm{O}$

$\mathrm{O}$

$\mathrm{O}$

C

$\mathrm{H}$

$\mathrm{H}$

$\mathrm{H}$

C

$\mathrm{H}$

$\mathrm{H}$

$\mathrm{H}$

C

$\mathrm{H}$

$\mathrm{H}$

$\mathrm{H}$
$-0.48160300-1.69987400$

$-1.79210200 \quad-3.23617800$

$-2.82491600-2.98438000$

$2.03717000 \quad-2.22810500$

$-3.14244900-1.85701200$

$2.32840100-1.53066300$

$2.90521100 \quad-2.03128300$

$3.45192300 \quad-0.72456900$

$1.64998500-1.59848400$

$4.04413600-1.25520600$

$2.65092800 \quad-2.46292700$

$4.31475700 \quad-0.61155600$

$3.67100900 \quad-0.19248100$

$4.71430600-1.12775300$

$-1.92628700 \quad-0.20937700$

$-2.98643300 \quad 0.18646200$

$-0.76829300-0.69864600$

$\begin{array}{ll}-0.62308800 & 1.33934600\end{array}$

$-2.61758800-0.35999100$

$0.21125800-1.49059000$

$0.37775600 \quad 1.10306300$

$-1.64136800-1.16693800$

$0.57084500-2.05780000$

$0.68590900 \quad-0.01972300$

$1.05932200-1.78775600$

$1.26174600-2.85954500$

$0.56647200-1.44480900$

$2.01982200-1.27302000$

$-3.74652300-4.15192700$

$-4.51177900 \quad-3.88580300$

$-3.16776700-5.00764700$

$-4.22487300 \quad-4.44424200$

$-3.38297500 \quad-0.01415600$

$\begin{array}{ll}-4.37661600 & 0.36023200\end{array}$

$-3.45281800-0.88916600$

$-2.83834900$

0.76608800
Enthalpy (a. u.)

Gibbs Free Energy (a. u.)
1.37646700

0.87912100

0.17006100

2.83416700

$-0.31292600$

4.03049200

1.73709600

4.14165100

4.87080200

1.84125900

0.78225000

3.05167500

5.05812000

1.00158600

0.04342600

1.77977500

$-1.60908500$

0.47736500

2.86402100

$-1.46999800$

1.22299100

3.00848400

$-0.38413400$

1.73701900

$-2.68356000$

$-2.74211800$

$-3.59305000$

$-2.57881100$

$-0.09063100$

$-0.81929500$

$-0.44658600$

0.84941700

4.11936500

3.87191400

4.76808100

4.66140800 


$\begin{array}{lrrr}\mathrm{C} & 1.28172800 & 2.26377000 & 1.56607500 \\ \mathrm{H} & 0.91093200 & 2.73922200 & 2.48041200 \\ \mathrm{H} & 2.29724100 & 1.91116200 & 1.75150800 \\ \mathrm{H} & 1.27177800 & 3.00445900 & 0.76542300 \\ \mathrm{C} & 0.16485300 & -3.60267500 & 3.85883600 \\ \mathrm{C} & -1.21429700 & -3.88556200 & 3.89491000 \\ \mathrm{C} & 0.93785200 & -4.04422700 & 4.96239200 \\ \mathrm{C} & -1.81044300 & -4.51532800 & 4.98267900 \\ \mathrm{H} & -1.82749100 & -3.60803100 & 3.05014300 \\ \mathrm{C} & 0.36250100 & -4.67392300 & 6.04974700 \\ \mathrm{H} & 2.01267400 & -3.90306100 & 4.95215700 \\ \mathrm{C} & -1.02539400 & -4.90487600 & 6.07666700 \\ \mathrm{H} & -2.87691100 & -4.70144600 & 4.96734000 \\ \mathrm{H} & 0.96294800 & -5.00937100 & 6.88853100 \\ \mathrm{C} & 0.76881700 & -2.95402800 & 2.68470700 \\ \mathrm{C} & 0.94900500 & -4.51895300 & 1.31137400 \\ \mathrm{C} & 1.93982500 & -5.15802900 & 2.03329000 \\ \mathrm{H} & 1.16893800 & -4.00843100 & 0.38196200 \\ \mathrm{H} & -0.08424300 & -4.81584500 & 1.43256500 \\ \mathrm{H} & 1.63859600 & -5.68547900 & 2.93427300 \\ \mathrm{C} & 3.36124000 & -5.07860000 & 1.80447500 \\ \mathrm{C} & 3.91044300 & -4.72727200 & 0.55215000 \\ \mathrm{C} & 4.23636500 & -5.31213800 & 2.88678800 \\ \mathrm{C} & 5.28389100 & -4.58769500 & 0.39944900 \\ \mathrm{H} & 3.25339400 & -4.57722200 & -0.29791900 \\ \mathrm{C} & 5.60908600 & -5.15341200 & 2.73507500 \\ \mathrm{H} & 3.82193900 & -5.59593300 & 3.84961800 \\ \mathrm{C} & 6.13531100 & -4.78830600 & 1.49187600 \\ \mathrm{H} & 5.69602800 & -4.32060100 & -0.56812200 \\ \mathrm{H} & 6.27060300 & -5.31519100 & 3.57980500 \\ \mathrm{H} & 7.20732900 & -4.66883500 & 1.37155400 \\ \mathrm{O} & -1.49966600 & -5.51901000 & 7.18573200 \\ \mathrm{C} & -2.90044900 & -5.78440400 & 7.26481700 \\ \mathrm{H} & -3.05936200 & -6.26297200 & 8.23078000 \\ \mathrm{H} & -3.48157900 & -4.85735300 & 7.21467900 \\ \mathrm{H} & -3.22147800 & -6.45971000 & 6.46436000 \\ \mathrm{~N} & 5.50768000 & 0.20897700 & 3.17014700 \\ \mathrm{O} & 6.26702000 & 0.28028200 & 2.19727200 \\ \mathrm{O} & 5.71055900 & 0.79803500 & 4.23776800\end{array}$




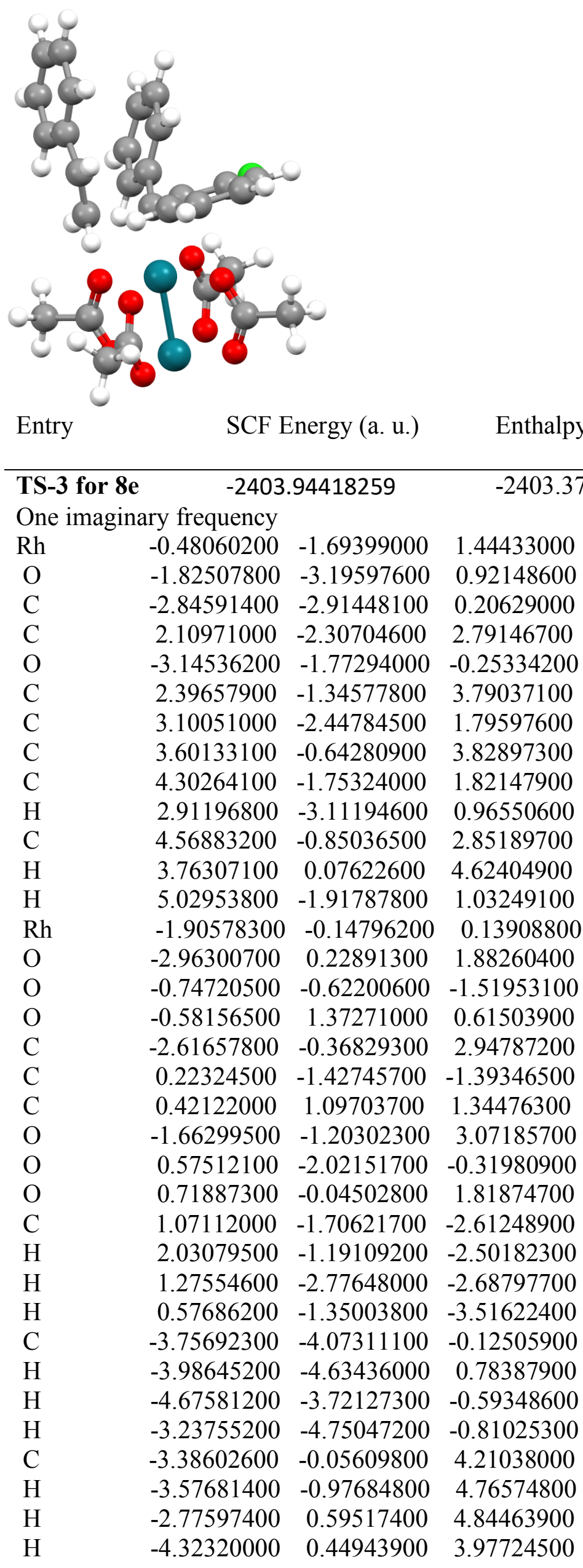




\begin{tabular}{lrrr}
$\mathrm{C}$ & 1.34548200 & 2.23485800 & 1.71233100 \\
$\mathrm{H}$ & 0.86750600 & 2.84114000 & 2.48875600 \\
$\mathrm{H}$ & 2.29109900 & 1.85037800 & 2.09509100 \\
$\mathrm{H}$ & 1.51511600 & 2.87562500 & 0.84458900 \\
$\mathrm{C}$ & 0.21804100 & -3.62841900 & 3.86317100 \\
$\mathrm{C}$ & -1.15926000 & -3.93105700 & 3.93218900 \\
$\mathrm{C}$ & 1.03238900 & -4.03095000 & 4.94632700 \\
$\mathrm{C}$ & -1.70322600 & -4.53530400 & 5.06168800 \\
$\mathrm{H}$ & -1.79296500 & -3.67701600 & 3.09446200 \\
$\mathrm{C}$ & 0.48967100 & -4.64008100 & 6.07033700 \\
$\mathrm{H}$ & 2.09999700 & -3.84889200 & 4.89909200 \\
$\mathrm{C}$ & -0.88478700 & -4.88378200 & 6.13817700 \\
$\mathrm{H}$ & -2.76796200 & -4.74509400 & 5.09786300 \\
$\mathrm{H}$ & 1.13759900 & -4.93581400 & 6.88911600 \\
$\mathrm{H}$ & 5.50567500 & -0.30453800 & 2.89004600 \\
$\mathrm{C}$ & 0.77823200 & -2.96237100 & 2.68111700 \\
$\mathrm{C}$ & 0.85614300 & -4.52901700 & 1.29839900 \\
$\mathrm{C}$ & 1.78530100 & -5.33265500 & 1.93530100 \\
$\mathrm{H}$ & 1.14858100 & -3.94953800 & 0.43186600 \\
$\mathrm{H}$ & -0.20080000 & -4.75663900 & 1.34444400 \\
$\mathrm{H}$ & 2.83909900 & -5.13580000 & 1.75810400 \\
$\mathrm{C}$ & 1.50508200 & -6.30991600 & 2.94731200 \\
$\mathrm{C}$ & 2.57111600 & -6.77974500 & 3.75027200 \\
$\mathrm{C}$ & 0.19676700 & -6.76773600 & 3.22177600 \\
$\mathrm{C}$ & 2.33295600 & -7.64122800 & 4.81169700 \\
$\mathrm{H}$ & 3.57916600 & -6.43475400 & 3.54010200 \\
$\mathrm{C}$ & -0.03650400 & -7.62619400 & 4.28614300 \\
$\mathrm{H}$ & -0.62886100 & -6.44461700 & 2.60054800 \\
$\mathrm{C}$ & 1.02542100 & -8.05601700 & 5.08882700 \\
$\mathrm{H}$ & 3.15579000 & -7.98330000 & 5.43075300 \\
$\mathrm{H}$ & -1.04672800 & -7.95804200 & 4.50031200 \\
$\mathrm{H}$ & 0.83557700 & -8.72275300 & 5.92415600 \\
$\mathrm{H}$ & -1.31101700 & -5.35821200 & 7.01689000 \\
$\mathrm{Cl}$ & 1.21335300 & -0.87113000 & 5.01023000 \\
& & & \\
\hline & & &
\end{tabular}




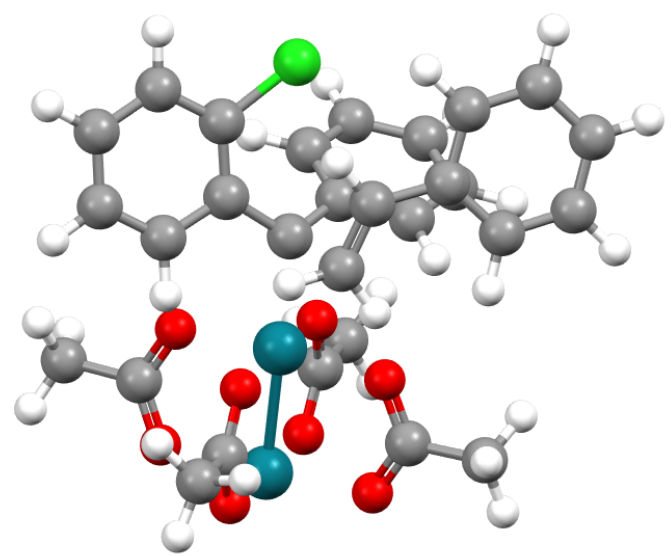

Entry SCF Energy (a. u.)

Enthalpy (a. u.)

Gibbs Free Energy (a. u.)

\begin{tabular}{|c|c|c|c|c|}
\hline \multirow[b]{2}{*}{ TS-4 for $8 \mathrm{e}$} & \multirow{2}{*}{\multicolumn{2}{|c|}{ e $\quad-2403.93444089$}} & & \\
\hline & & & -2403.367277 & -2403.485693 \\
\hline \multicolumn{5}{|c|}{ One imaginary frequency } \\
\hline $\mathrm{Rh}$ & -0.43170000 & -1.69790000 & 1.47550000 & \\
\hline $\mathrm{O}$ & -1.70770000 & -3.27920000 & 1.03810000 & \\
\hline $\mathrm{C}$ & -2.66010000 & -3.11330000 & 0.20280000 & \\
\hline $\mathrm{C}$ & 2.02590000 & -2.09990000 & 3.06880000 & \\
\hline $\mathrm{O}$ & -2.94320000 & -2.03810000 & -0.40370000 & \\
\hline $\mathrm{C}$ & 2.23350000 & -1.34980000 & 4.24670000 & \\
\hline $\mathrm{C}$ & 2.93620000 & -1.92470000 & 2.00950000 & \\
\hline $\mathrm{C}$ & 3.31880000 & -0.48780000 & 4.36360000 & \\
\hline $\mathrm{H}$ & 1.52600000 & -1.43280000 & 5.06430000 & \\
\hline $\mathrm{C}$ & 4.04760000 & -1.10170000 & 2.14680000 & \\
\hline $\mathrm{H}$ & 2.74640000 & -2.41630000 & 1.06870000 & \\
\hline $\mathrm{C}$ & 4.24690000 & -0.37580000 & 3.32470000 & \\
\hline $\mathrm{H}$ & 3.44920000 & 0.08710000 & 5.27560000 & \\
\hline $\mathrm{H}$ & 4.74980000 & -1.01090000 & 1.32320000 & \\
\hline $\mathrm{Rh}$ & -1.80720000 & -0.32990000 & -0.06050000 & \\
\hline $\mathrm{O}$ & -3.01080000 & 0.14470000 & 1.55890000 & \\
\hline $\mathrm{O}$ & -0.51580000 & -0.87920000 & -1.58900000 & \\
\hline $\mathrm{O}$ & -0.58370000 & 1.28330000 & 0.36860000 & \\
\hline $\mathrm{C}$ & -2.68210000 & -0.27440000 & 2.71000000 & \\
\hline $\mathrm{C}$ & 0.44280000 & -1.66730000 & -1.33610000 & \\
\hline $\mathrm{C}$ & 0.37970000 & 1.11340000 & 1.18010000 & \\
\hline $\mathrm{O}$ & -1.68480000 & -1.01940000 & 2.98670000 & \\
\hline $\mathrm{O}$ & 0.72000000 & -2.17140000 & -0.19630000 & \\
\hline $\mathrm{O}$ & 0.68860000 & 0.02950000 & 1.76940000 & \\
\hline $\mathrm{C}$ & 1.34800000 & -2.07680000 & -2.47350000 & \\
\hline $\mathrm{H}$ & 1.19210000 & -3.13870000 & -2.68800000 & \\
\hline $\mathrm{H}$ & 1.13620000 & -1.49180000 & -3.36810000 & \\
\hline $\mathrm{H}$ & 2.39210000 & -1.94940000 & -2.17690000 & \\
\hline $\mathrm{C}$ & -3.50310000 & -4.33250000 & -0.08710000 & \\
\hline $\mathrm{H}$ & -2.90750000 & -5.04860000 & -0.66200000 & \\
\hline $\mathrm{H}$ & -3.78900000 & -4.81650000 & 0.84970000 & \\
\hline $\mathrm{H}$ & -4.39060000 & -4.06140000 & -0.65850000 & \\
\hline $\mathrm{C}$ & -3.52710000 & 0.16220000 & 3.88370000 & \\
\hline $\mathrm{H}$ & -3.61570000 & -0.65190000 & 4.60540000 & \\
\hline $\mathrm{H}$ & -3.03180000 & 1.00220000 & 4.38200000 & \\
\hline $\mathrm{H}$ & -4.51260000 & 0.48580000 & 3.54790000 & \\
\hline $\mathrm{C}$ & 1.23240000 & 2.31700000 & 1.50910000 & \\
\hline $\mathrm{H}$ & 0.78560000 & 2.84180000 & 2.36030000 & \\
\hline & & & S113 & \\
\hline
\end{tabular}




$\begin{array}{lrrr}\mathrm{H} & 2.23870000 & 2.00340000 & 1.78980000 \\ \mathrm{H} & 1.26530000 & 3.00400000 & 0.66230000 \\ \mathrm{C} & 0.16000000 & -3.50440000 & 4.08000000 \\ \mathrm{C} & -1.24950000 & -3.62590000 & 4.10970000 \\ \mathrm{C} & 0.83910000 & -4.07550000 & 5.19080000 \\ \mathrm{C} & -1.93830000 & -4.17700000 & 5.18240000 \\ \mathrm{H} & -1.80100000 & -3.26610000 & 3.25680000 \\ \mathrm{C} & 0.15800000 & -4.62880000 & 6.27400000 \\ \mathrm{C} & -1.23450000 & -4.66540000 & 6.28200000 \\ \mathrm{H} & -3.02170000 & -4.22940000 & 5.15480000 \\ \mathrm{H} & 0.72500000 & -5.04510000 & 7.09840000 \\ \mathrm{H} & 5.10690000 & 0.27900000 & 3.42710000 \\ \mathrm{H} & -1.75510000 & -5.09640000 & 7.13100000 \\ \mathrm{C} & 0.77650000 & -2.84740000 & 2.90060000 \\ \mathrm{C} & 0.93830000 & -4.55840000 & 1.59460000 \\ \mathrm{C} & 2.01710000 & -5.20030000 & 2.15830000 \\ \mathrm{H} & 1.02830000 & -4.04950000 & 0.64490000 \\ \mathrm{H} & -0.06470000 & -4.85790000 & 1.86330000 \\ \mathrm{H} & 1.85450000 & -5.77950000 & 3.06220000 \\ \mathrm{C} & 3.38320000 & -5.14440000 & 1.68660000 \\ \mathrm{C} & 3.72300000 & -4.63840000 & 0.41200000 \\ \mathrm{C} & 4.41910000 & -5.62140000 & 2.51670000 \\ \mathrm{C} & 5.04850000 & -4.59020000 & 0.00000000 \\ \mathrm{H} & 2.94200000 & -4.29240000 & -0.25630000 \\ \mathrm{C} & 5.74570000 & -5.56960000 & 2.10310000 \\ \mathrm{H} & 4.16830000 & -6.01890000 & 3.49470000 \\ \mathrm{C} & 6.06490000 & -5.04890000 & 0.84560000 \\ \mathrm{H} & 5.29390000 & -4.19900000 & -0.98220000 \\ \mathrm{H} & 6.53170000 & -5.93210000 & 2.75770000 \\ \mathrm{H} & 7.10010000 & -5.00720000 & 0.52160000 \\ \mathrm{Cl} & 2.59310000 & -4.24050000 & 5.26330000\end{array}$




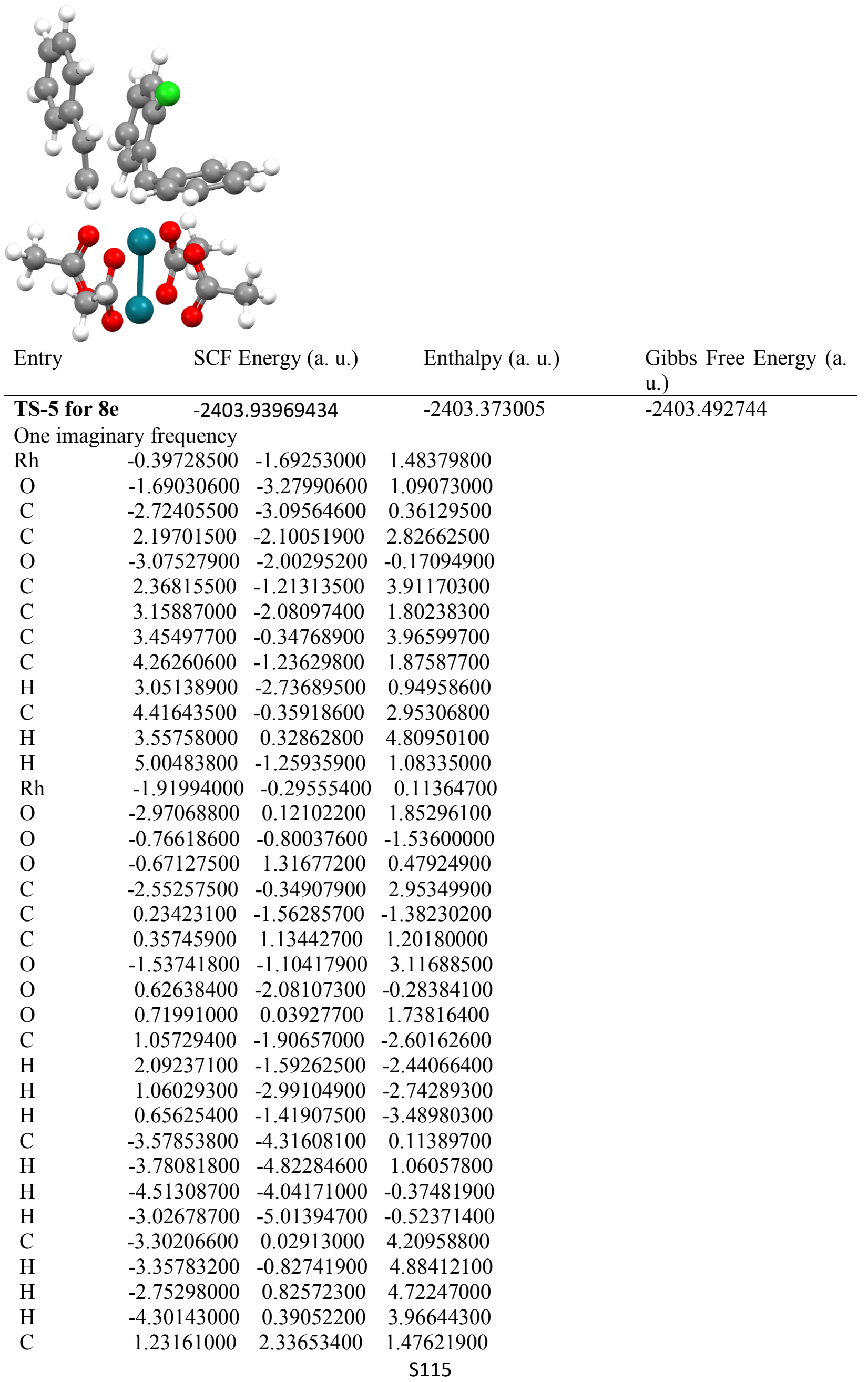




$\begin{array}{lrrr}\mathrm{H} & 0.72672000 & 2.98318600 & 2.20126700 \\ \mathrm{H} & 2.19357700 & 2.02634800 & 1.88447400 \\ \mathrm{H} & 1.37310600 & 2.91157400 & 0.55859100 \\ \mathrm{C} & 0.43047400 & -3.53712900 & 3.93828400 \\ \mathrm{C} & -0.96377000 & -3.75673900 & 4.06625100 \\ \mathrm{C} & 1.23176300 & -4.09292100 & 4.97948100 \\ \mathrm{C} & -1.53009200 & -4.38446300 & 5.16752300 \\ \mathrm{H} & -1.59974200 & -3.41195500 & 3.26723900 \\ \mathrm{C} & 0.67087700 & -4.72733200 & 6.08397800 \\ \mathrm{C} & -0.71217800 & -4.86121800 & 6.18963800 \\ \mathrm{H} & -2.60610900 & -4.51352400 & 5.21811900 \\ \mathrm{H} & 1.32288200 & -5.13385500 & 6.84771100 \\ \mathrm{H} & 5.27470100 & 0.30366600 & 3.00263700 \\ \mathrm{C} & 0.91932400 & -2.82238000 & 2.75461300 \\ \mathrm{C} & 1.10144800 & -4.52849000 & 1.28124700 \\ \mathrm{C} & 1.88619800 & -5.40251900 & 1.98071700 \\ \mathrm{H} & 1.52018500 & -3.89681700 & 0.51160500 \\ \mathrm{H} & 0.02630900 & -4.64137900 & 1.23936000 \\ \mathrm{H} & 2.96689000 & -5.30104500 & 1.91256000 \\ \mathrm{C} & 1.40117200 & -6.41547400 & 2.89305300 \\ \mathrm{C} & 2.32885200 & -7.14367600 & 3.66863800 \\ \mathrm{C} & 0.02528300 & -6.67446300 & 3.07264100 \\ \mathrm{C} & 1.89928200 & -8.06745200 & 4.61216300 \\ \mathrm{H} & 3.38887500 & -6.95115100 & 3.53712500 \\ \mathrm{C} & -0.40206200 & -7.59596100 & 4.02002000 \\ \mathrm{H} & -0.70784600 & -6.14494300 & 2.47687400 \\ \mathrm{C} & 0.53003000 & -8.28793800 & 4.79875100 \\ \mathrm{H} & 2.62455400 & -8.60978000 & 5.21024000 \\ \mathrm{H} & -1.46368100 & -7.77140600 & 4.15962200 \\ \mathrm{H} & 0.19202500 & -9.00213300 & 5.54316400 \\ \mathrm{H} & -1.13678600 & -5.35809200 & 7.05582800 \\ \mathrm{Cl} & 2.99098400 & -4.10091900 & 4.93668400 \\ \mathrm{H} & 1.62709400 & -1.19850400 & 4.70375800\end{array}$




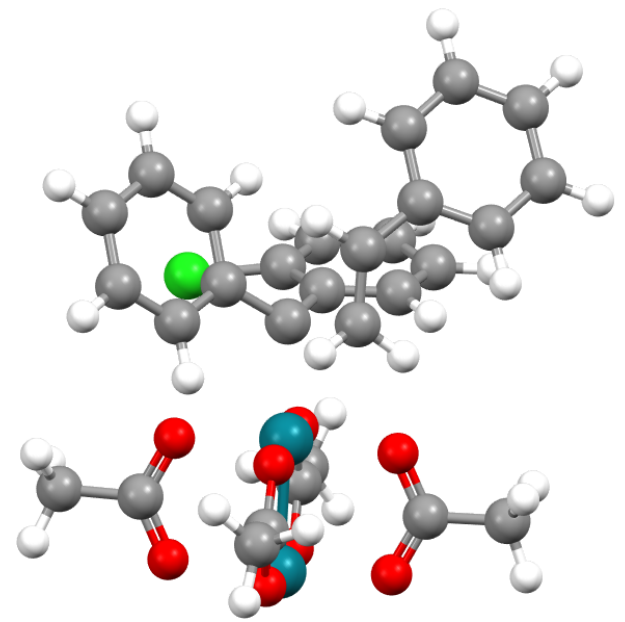

Entry SCF Energy (a. u.) Enthalpy (a. u.) $\quad$ Gibbs Free Energy (a.

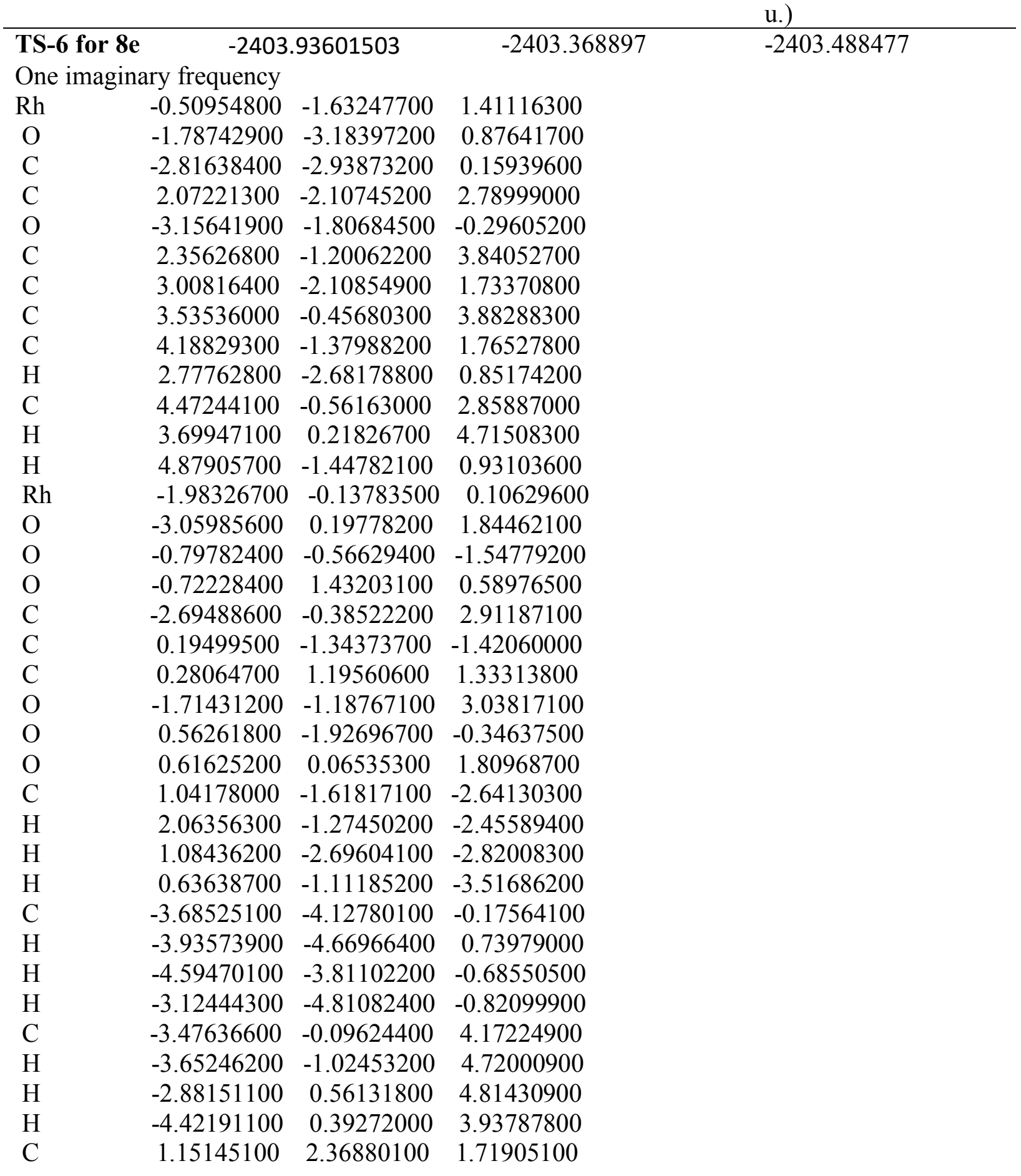




$\begin{array}{lrrr}\mathrm{H} & 0.69144000 & 2.88280000 & 2.56970400 \\ \mathrm{H} & 2.14358200 & 2.02626500 & 2.01472800 \\ \mathrm{H} & 1.21947800 & 3.07767600 & 0.89205900 \\ \mathrm{C} & 0.24598700 & -3.53876400 & 3.86360200 \\ \mathrm{C} & -1.10753400 & -3.91743700 & 3.90478600 \\ \mathrm{C} & 1.07014400 & -3.91430200 & 4.94527400 \\ \mathrm{C} & -1.62514400 & -4.60445200 & 5.00239600 \\ \mathrm{H} & -1.74755000 & -3.67264100 & 3.06879700 \\ \mathrm{C} & 0.55680200 & -4.60325600 & 6.03730600 \\ \mathrm{H} & 2.12629700 & -3.66782200 & 4.91616200 \\ \mathrm{C} & -0.79972400 & -4.94499100 & 6.07452700 \\ \mathrm{H} & -2.67554400 & -4.87962900 & 5.01570800 \\ \mathrm{H} & 1.21200600 & -4.87964800 & 6.85775900 \\ \mathrm{H} & 5.39192300 & 0.01256500 & 2.90489100 \\ \mathrm{H} & -1.20346600 & -5.48297000 & 6.92691000 \\ \mathrm{C} & 0.79862100 & -2.85364900 & 2.66634400 \\ \mathrm{C} & 0.93639800 & -4.39631300 & 1.32712900 \\ \mathrm{C} & 1.83752100 & -5.14913000 & 2.06794200 \\ \mathrm{H} & 1.23085100 & -3.91536100 & 0.40251300 \\ \mathrm{H} & -0.11767700 & -4.63270900 & 1.38887800 \\ \mathrm{H} & 1.44692900 & -5.65349700 & 2.94673900 \\ \mathrm{C} & 3.26333900 & -5.23008100 & 1.90191600 \\ \mathrm{C} & 3.91047300 & -4.93589000 & 0.68101900 \\ \mathrm{C} & 4.04983700 & -5.59449600 & 3.01719400 \\ \mathrm{C} & 5.29527500 & -4.97637000 & 0.59185800 \\ \mathrm{H} & 3.31830400 & -4.69469500 & -0.19541200 \\ \mathrm{C} & 5.43652400 & -5.61692400 & 2.92853400 \\ \mathrm{H} & 3.55563400 & -5.83463000 & 3.95380700 \\ \mathrm{C} & 6.06134000 & -5.30491700 & 1.71678300 \\ \mathrm{H} & 5.78361700 & -4.75352400 & -0.35104600 \\ \mathrm{H} & 6.03221200 & -5.87909400 & 3.79666500 \\ \mathrm{H} & 7.14409300 & -5.32805800 & 1.64486100 \\ \mathrm{Cl} & 1.17977500 & -0.80802400 & 5.09720100\end{array}$




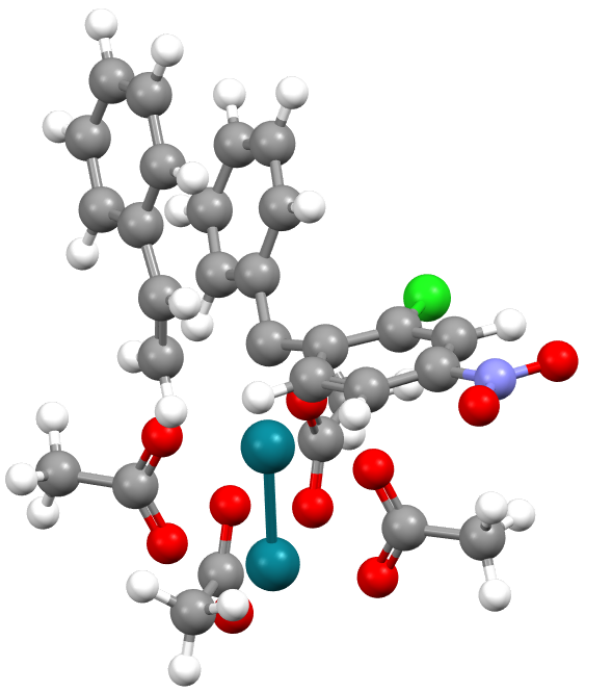

Entry SCF Energy (a. u.) Enthalpy (a. u.) $\quad$ Gibbs Free Energy (a. u.)

\begin{tabular}{|c|c|c|c|}
\hline \multirow{2}{*}{\multicolumn{2}{|c|}{$\begin{array}{l}\text { TS-3 for } 8 f \quad-2608 \\
\text { One imaginary frequency }\end{array}$}} & & \multirow[t]{2}{*}{-2607.87} \\
\hline & & \multirow[b]{2}{*}{-1.67143600} & \\
\hline \multicolumn{2}{|c|}{ Rh $\quad-0.46950100$} & & 1.46932100 \\
\hline \multirow{2}{*}{$\begin{array}{l}\mathrm{O} \\
\mathrm{C}\end{array}$} & -1.80728300 & -3.17560800 & 0.94378900 \\
\hline & -2.82156600 & -2.90118500 & 0.21584800 \\
\hline \multirow{2}{*}{$\begin{array}{l}\mathrm{C} \\
\mathrm{O}\end{array}$} & 2.08021000 & -2.29030400 & 2.80299900 \\
\hline & -3.11635000 & -1.76328100 & -0.25522400 \\
\hline $\mathrm{C}$ & 2.36860300 & -1.31801400 & 3.79952000 \\
\hline $\mathrm{C}$ & 3.05915600 & -2.44272300 & 1.79482700 \\
\hline \multirow{2}{*}{$\begin{array}{l}\mathrm{C} \\
\mathrm{C}\end{array}$} & 3.56326500 & -0.61204400 & 3.82204800 \\
\hline & 4.25615300 & -1.75000700 & 1.79526700 \\
\hline $\mathrm{H}$ & 2.86023100 & -3.11240600 & 0.97344700 \\
\hline $\mathrm{C}$ & 4.50381100 & -0.84293000 & 2.82441300 \\
\hline \multirow{2}{*}{$\begin{array}{l}\mathrm{H} \\
\mathrm{H}\end{array}$} & 3.76028200 & 0.11793400 & 4.59482500 \\
\hline & 4.98964800 & -1.89900300 & 1.01429900 \\
\hline $\mathrm{Rh}$ & -1.87883900 & -0.14044700 & 0.13853400 \\
\hline $\mathrm{O}$ & -2.95198700 & 0.25035100 & 1.86773100 \\
\hline \multirow{2}{*}{$\begin{array}{l}\mathrm{O} \\
\mathrm{O}\end{array}$} & -0.70486900 & -0.62611600 & -1.50408800 \\
\hline & -0.55834700 & 1.38322900 & 0.61224800 \\
\hline $\mathrm{C}$ & -2.62103300 & -0.34053300 & 2.94076600 \\
\hline \multirow{2}{*}{$\begin{array}{l}\mathrm{C} \\
\mathrm{C}\end{array}$} & 0.26722600 & -1.42592300 & -1.36085800 \\
\hline & 0.43578000 & 1.11810800 & 1.35552400 \\
\hline $\mathrm{O}$ & -1.67013600 & -1.17763500 & 3.08008300 \\
\hline $\mathrm{O}$ & 0.61230100 & -2.00435000 & -0.27547600 \\
\hline $\mathrm{O}$ & 0.72547500 & -0.01985900 & 1.84823500 \\
\hline $\mathrm{C}$ & 1.11621600 & -1.73600400 & -2.57069100 \\
\hline $\mathrm{H}$ & 2.14189600 & -1.40388100 & -2.38565700 \\
\hline $\mathrm{H}$ & 1.14368900 & -2.81739900 & -2.72941000 \\
\hline $\mathrm{H}$ & 0.72329700 & -1.23917800 & -3.45725400 \\
\hline \multirow{2}{*}{$\begin{array}{l}\mathrm{C} \\
\mathrm{H}\end{array}$} & -3.72712400 & -4.06316500 & -0.11565400 \\
\hline & -3.95951900 & -4.62196600 & 0.79396500 \\
\hline $\mathrm{H}$ & -4.64427900 & -3.71519500 & -0.59022900 \\
\hline $\mathrm{H}$ & -3.20193100 & -4.74085700 & -0.79606400 \\
\hline $\mathrm{C}$ & -3.40390000 & -0.01993500 & 4.19199500 \\
\hline $\mathrm{H}$ & -3.61349400 & -0.93865100 & 4.74397100 \\
\hline $\mathrm{H}$ & -2.79486400 & 0.62352300 & 4.83503700 \\
\hline $\mathrm{H}$ & -4.33161600 & 0.49639600 & 3.94551100 \\
\hline & & & S119 \\
\hline
\end{tabular}




$\begin{array}{lrrr}\mathrm{C} & 1.35711900 & 2.25598800 & 1.72665100 \\ \mathrm{H} & 0.96693800 & 2.74337700 & 2.62647300 \\ \mathrm{H} & 2.35680600 & 1.87899100 & 1.94621300 \\ \mathrm{H} & 1.39136200 & 2.99473300 & 0.92471100 \\ \mathrm{C} & 0.20880100 & -3.62784400 & 3.86599400 \\ \mathrm{C} & -1.17183500 & -3.92312300 & 3.94673700 \\ \mathrm{C} & 1.04021400 & -4.05925300 & 4.92793400 \\ \mathrm{C} & -1.70024300 & -4.54882800 & 5.06981800 \\ \mathrm{H} & -1.81412900 & -3.64512900 & 3.12352100 \\ \mathrm{C} & 0.50964300 & -4.68648200 & 6.04646700 \\ \mathrm{H} & 2.10786500 & -3.88094300 & 4.86904100 \\ \mathrm{C} & -0.86556000 & -4.92344300 & 6.12625000 \\ \mathrm{H} & -2.76495200 & -4.75426400 & 5.11976900 \\ \mathrm{H} & 1.16588900 & -5.00347300 & 6.84994100 \\ \mathrm{C} & 0.74929500 & -2.92392800 & 2.71408300 \\ \mathrm{C} & 0.84189400 & -4.59512200 & 1.25777700 \\ \mathrm{C} & 1.77474800 & -5.37488300 & 1.89521500 \\ \mathrm{H} & 1.11752700 & -3.98586100 & 0.40671900 \\ \mathrm{H} & -0.21856600 & -4.78603600 & 1.35186700 \\ \mathrm{H} & 2.82723900 & -5.19301300 & 1.69282700 \\ \mathrm{C} & 1.50295000 & -6.33401700 & 2.93378400 \\ \mathrm{C} & 2.57253800 & -6.78012700 & 3.74356100 \\ \mathrm{C} & 0.19960300 & -6.79715700 & 3.21736000 \\ \mathrm{C} & 2.34205700 & -7.62418300 & 4.82150200 \\ \mathrm{H} & 3.57791200 & -6.43176300 & 3.52613700 \\ \mathrm{C} & -0.02623200 & -7.64009200 & 4.29594100 \\ \mathrm{H} & -0.62921900 & -6.49289700 & 2.59032000 \\ \mathrm{C} & 1.03912200 & -8.04643400 & 5.10637400 \\ \mathrm{H} & 3.16846100 & -7.94755500 & 5.44585900 \\ \mathrm{H} & -1.03345900 & -7.97806900 & 4.51489900 \\ \mathrm{H} & 0.85539300 & -8.70073100 & 5.95278800 \\ \mathrm{H} & -1.28187800 & -5.41352300 & 7.00092500 \\ \mathrm{Cl} & 1.19954900 & -0.86420700 & 5.02791300 \\ \mathrm{~N} & 5.75692600 & -0.10597100 & 2.85213900 \\ \mathrm{O} & 6.57864100 & -0.32710500 & 1.95742800 \\ \mathrm{O} & 5.93798900 & 0.70415300 & 3.76648900\end{array}$




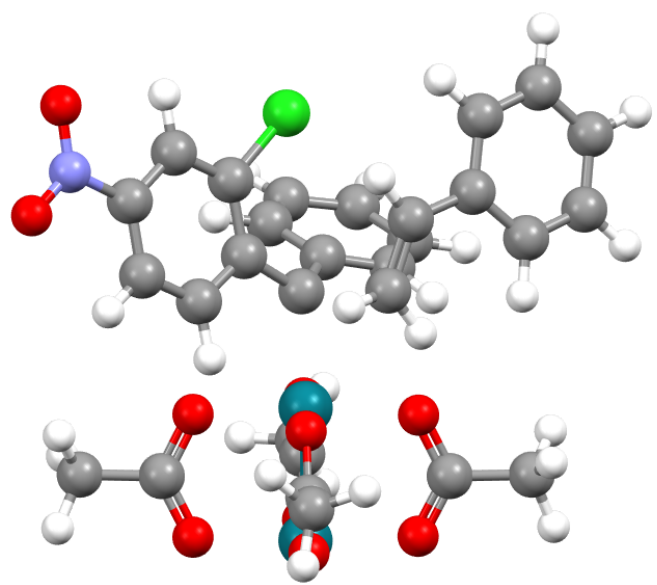

Entry SCF Energy (a. u.)

Enthalpy (a. u.)

Gibbs Free Energy (a. u.)

\begin{tabular}{lrcc}
\hline TS-4 for 8f & -2608.43597222 & -2607.8638 \\
One imaginary frequency & & \\
$\mathrm{Rh}$ & -0.42798400 & -1.69845200 & 1.48282300 \\
$\mathrm{O}$ & -1.71112200 & -3.27955700 & 1.07553500 \\
$\mathrm{C}$ & -2.65839600 & -3.12419600 & 0.23117800 \\
$\mathrm{C}$ & 2.01263400 & -2.08627200 & 3.10151900 \\
$\mathrm{O}$ & -2.93087600 & -2.05909500 & -0.39658400 \\
$\mathrm{C}$ & 2.24019600 & -1.36788500 & 4.29604000 \\
$\mathrm{C}$ & 2.91871300 & -1.90613400 & 2.03843300 \\
$\mathrm{C}$ & 3.34100400 & -0.52810400 & 4.42311700 \\
$\mathrm{H}$ & 1.53592300 & -1.45394000 & 5.11632700 \\
$\mathrm{C}$ & 4.04939100 & -1.11310200 & 2.18905400 \\
$\mathrm{H}$ & 2.71050200 & -2.37415000 & 1.08941200 \\
$\mathrm{C}$ & 4.26624000 & -0.41577400 & 3.38113100 \\
$\mathrm{H}$ & 3.48711800 & 0.02743400 & 5.34441900 \\
$\mathrm{H}$ & 4.75098700 & -1.01990500 & 1.36559500 \\
$\mathrm{Rh}$ & -1.79103400 & -0.34844100 & -0.07699500 \\
$\mathrm{O}$ & -2.99814600 & 0.15607900 & 1.52981500 \\
$\mathrm{O}$ & -0.49709100 & -0.92581100 & -1.59223000 \\
$\mathrm{O}$ & -0.56204500 & 1.26512700 & 0.33302300 \\
$\mathrm{C}$ & -2.67546800 & -0.24377000 & 2.68839300 \\
$\mathrm{C}$ & 0.45320100 & -1.71861400 & -1.32627000 \\
$\mathrm{C}$ & 0.39967700 & 1.10321800 & 1.14736400 \\
$\mathrm{O}$ & -1.67945200 & -0.98708900 & 2.98001100 \\
$\mathrm{O}$ & 0.72055800 & -2.21058100 & -0.17767400 \\
$\mathrm{O}$ & 0.70321800 & 0.02550600 & 1.75241600 \\
$\mathrm{C}$ & 1.36114100 & -2.14993200 & -2.45261800 \\
$\mathrm{H}$ & 1.20641400 & -3.21585000 & -2.64689400 \\
$\mathrm{H}$ & 1.15157800 & -1.58202800 & -3.35866900 \\
$\mathrm{H}$ & 2.40428900 & -2.01578600 & -2.15553800 \\
$\mathrm{C}$ & -3.50587500 & -4.34414800 & -0.03971400 \\
$\mathrm{H}$ & -2.91052700 & -5.07422100 & -0.59704400 \\
$\mathrm{H}$ & -3.79942000 & -4.80824100 & 0.90471700 \\
$\mathrm{H}$ & -4.38857500 & -4.07940500 & -0.62135100 \\
$\mathrm{C}$ & -3.52290500 & 0.21038600 & 3.85283200 \\
$\mathrm{H}$ & -3.65716900 & -0.61042300 & 4.56014300 \\
$\mathrm{H}$ & -3.00220600 & 1.02049000 & 4.37397800 \\
$\mathrm{H}$ & -4.48855300 & 0.57739700 & 3.50498800 \\
$\mathrm{C}$ & 1.25815700 & 2.30633900 & 1.46074500
\end{tabular}




$\begin{array}{lrrr}\mathrm{H} & 0.81903000 & 2.83878800 & 2.31119800 \\ \mathrm{H} & 2.26529300 & 1.99121000 & 1.73707300 \\ \mathrm{H} & 1.28752300 & 2.98632800 & 0.60829200 \\ \mathrm{C} & 0.13793800 & -3.48640400 & 4.08892600 \\ \mathrm{C} & -1.27315000 & -3.58021700 & 4.12639100 \\ \mathrm{C} & 0.82456000 & -4.09948400 & 5.17603700 \\ \mathrm{C} & -1.96937500 & -4.14760100 & 5.18239100 \\ \mathrm{H} & -1.82690700 & -3.18623800 & 3.29132300 \\ \mathrm{C} & 0.14793300 & -4.67436300 & 6.24705700 \\ \mathrm{C} & -1.24105200 & -4.67362100 & 6.24259600 \\ \mathrm{H} & -3.05017000 & -4.18957000 & 5.18683600 \\ \mathrm{H} & 0.68772100 & -5.12789800 & 7.06647500 \\ \mathrm{H} & 5.13952100 & 0.21944700 & 3.49294000 \\ \mathrm{C} & 0.75502200 & -2.80766400 & 2.91765900 \\ \mathrm{C} & 0.92674600 & -4.60793200 & 1.62281300 \\ \mathrm{C} & 2.02561200 & -5.23203500 & 2.15870900 \\ \mathrm{H} & 0.98738900 & -4.07765200 & 0.68301500 \\ \mathrm{H} & -0.06639000 & -4.91040100 & 1.92316300 \\ \mathrm{H} & 1.89085600 & -5.83731800 & 3.05004300 \\ \mathrm{C} & 3.38372200 & -5.13003900 & 1.67002600 \\ \mathrm{C} & 3.69343500 & -4.58431300 & 0.40420000 \\ \mathrm{C} & 4.44225900 & -5.59633000 & 2.47734300 \\ \mathrm{C} & 5.01210900 & -4.48860700 & -0.02118500 \\ \mathrm{H} & 2.89538200 & -4.24400600 & -0.24642600 \\ \mathrm{C} & 5.76193600 & -5.49517500 & 2.05113700 \\ \mathrm{H} & 4.21489800 & -6.02602800 & 3.44754200 \\ \mathrm{C} & 6.05109900 & -4.93651200 & 0.80259800 \\ \mathrm{H} & 5.23466100 & -4.06789600 & -0.99651100 \\ \mathrm{H} & 6.56562800 & -5.84941600 & 2.68845600 \\ \mathrm{H} & 7.08078100 & -4.85687000 & 0.46859600 \\ \mathrm{Cl} & 2.56933300 & -4.28028600 & 5.23828900 \\ \mathrm{~N} & -1.94749400 & -5.27372200 & 7.37824200 \\ \mathrm{O} & -1.27316500 & -5.74494200 & 8.29487400 \\ \mathrm{O} & -3.17843500 & -5.27203100 & 7.35204800 \\ & & & \end{array}$




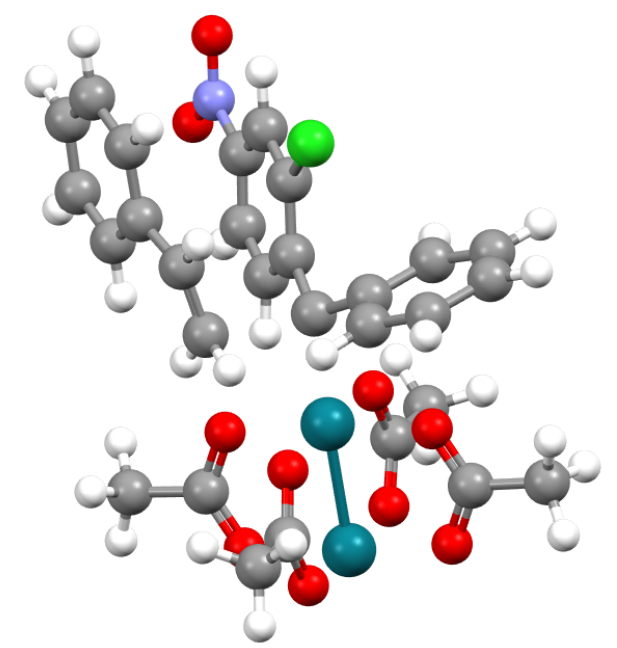

Entry SCF Energy (a. u.) $\quad$ Enthalpy (a. u.) $\quad$ Gibbs Free Energy (a.

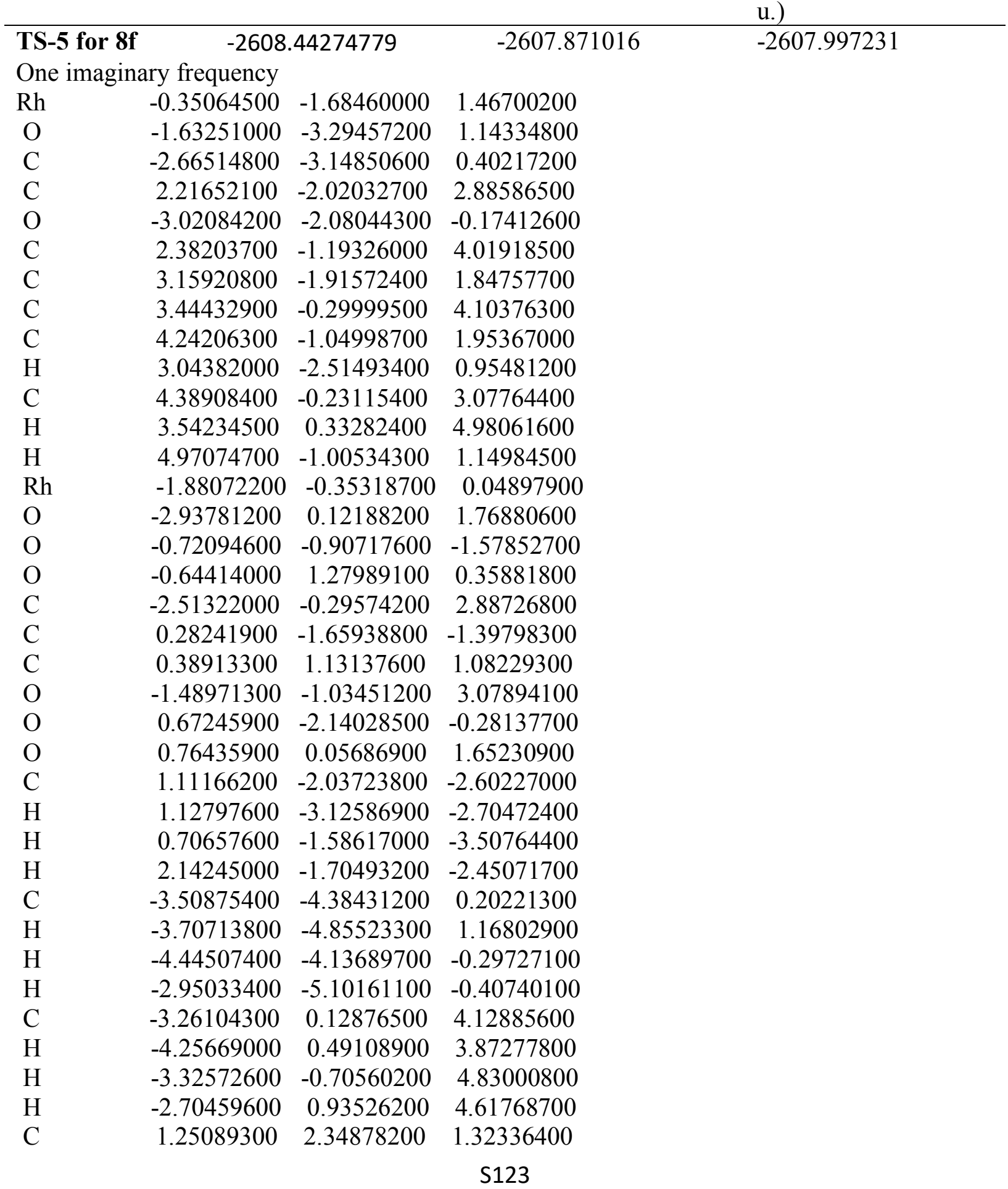




$\begin{array}{lrrr}\mathrm{H} & 1.25891100 & 2.98801300 & 0.43896300 \\ \mathrm{H} & 0.82452700 & 2.92173100 & 2.15367800 \\ \mathrm{H} & 2.26495500 & 2.05076800 & 1.59197000 \\ \mathrm{C} & 0.46064100 & -3.49137100 & 3.95900100 \\ \mathrm{C} & -0.93473700 & -3.68110400 & 4.09715100 \\ \mathrm{C} & 1.27046400 & -4.10854500 & 4.95870800 \\ \mathrm{C} & -1.50484900 & -4.37679300 & 5.15220200 \\ \mathrm{H} & -1.57762700 & -3.27081400 & 3.33715900 \\ \mathrm{C} & 0.72052300 & -4.82555600 & 6.01437800 \\ \mathrm{C} & -0.66037000 & -4.94577900 & 6.09698700 \\ \mathrm{H} & -2.57575900 & -4.50781500 & 5.22653500 \\ \mathrm{H} & 1.35161600 & -5.31118000 & 6.74459700 \\ \mathrm{H} & 5.23011300 & 0.45109400 & 3.15170700 \\ \mathrm{C} & 0.95823400 & -2.76322000 & 2.77495000 \\ \mathrm{C} & 1.18801900 & -4.50066000 & 1.35570100 \\ \mathrm{C} & 1.89430700 & -5.42390900 & 2.08142400 \\ \mathrm{H} & 1.67973000 & -3.89468700 & 0.60957100 \\ \mathrm{H} & 0.11421800 & -4.57422200 & 1.24636300 \\ \mathrm{H} & 2.98031400 & -5.37562600 & 2.06718600 \\ \mathrm{C} & 1.31440000 & -6.42032200 & 2.95359700 \\ \mathrm{C} & 2.16336100 & -7.18397000 & 3.78600700 \\ \mathrm{C} & -0.08067800 & -6.62206500 & 3.04969400 \\ \mathrm{C} & 1.63925800 & -8.07749100 & 4.71038200 \\ \mathrm{H} & 3.23644600 & -7.03860200 & 3.71635600 \\ \mathrm{C} & -0.60118400 & -7.51779800 & 3.97484100 \\ \mathrm{H} & -0.75411700 & -6.06483200 & 2.40973400 \\ \mathrm{C} & 0.25298200 & -8.23687600 & 4.81714900 \\ \mathrm{H} & 2.30346100 & -8.64206700 & 5.35640900 \\ \mathrm{H} & -1.67550400 & -7.64873500 & 4.05254500 \\ \mathrm{H} & -0.15980300 & -8.92295500 & 5.54981400 \\ \mathrm{Cl} & 3.02266500 & -4.11940800 & 4.90449500 \\ \mathrm{H} & 1.65602400 & -1.24250100 & 4.82385000 \\ \mathrm{~N} & -1.22890700 & -5.74388500 & 7.18586700 \\ \mathrm{O} & -0.45283200 & -6.26479600 & 7.98816600 \\ \mathrm{O} & -2.45409800 & -5.85734400 & 7.23360600 \\ & & & \\ & & \end{array}$




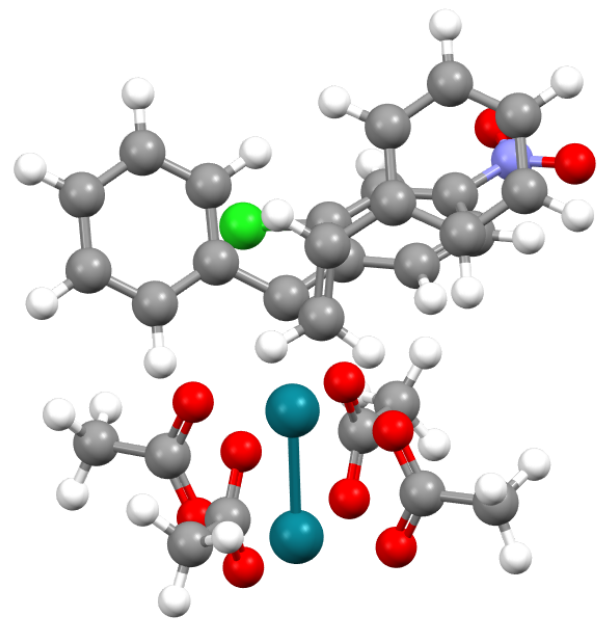

Entry SCF Energy (a. u.) Enthalpy (a. u.) Gibbs Free Energy (a.

\begin{tabular}{|c|c|c|c|c|}
\hline & & & & u.) \\
\hline TS-6 for $8 f$ & -2608 & .44283525 & -2607.870681 & -2607.996842 \\
\hline One imagin & ary frequency & & & \\
\hline $\mathrm{Rh}$ & -0.49970000 & -1.63130000 & 1.44320000 & \\
\hline $\mathrm{O}$ & -1.77450000 & -3.18320000 & 0.91170000 & \\
\hline $\mathrm{C}$ & -2.78790000 & -2.94650000 & 0.16940000 & \\
\hline $\mathrm{C}$ & 2.03220000 & -2.11810000 & 2.84150000 & \\
\hline $\mathrm{O}$ & -3.11270000 & -1.82090000 & -0.31200000 & \\
\hline $\mathrm{C}$ & 2.31610000 & -1.20150000 & 3.89060000 & \\
\hline $\mathrm{C}$ & 2.96640000 & -2.13930000 & 1.78130000 & \\
\hline $\mathrm{C}$ & 3.50050000 & -0.47890000 & 3.93680000 & \\
\hline $\mathrm{C}$ & 4.15490000 & -1.43540000 & 1.80500000 & \\
\hline $\mathrm{H}$ & 2.72430000 & -2.70790000 & 0.90070000 & \\
\hline $\mathrm{C}$ & 4.42450000 & -0.62560000 & 2.90690000 & \\
\hline $\mathrm{H}$ & 3.70130000 & 0.20190000 & 4.75230000 & \\
\hline $\mathrm{H}$ & 4.86080000 & -1.50030000 & 0.98810000 & \\
\hline $\mathrm{Rh}$ & -1.93710000 & -0.15540000 & 0.08640000 & \\
\hline $\mathrm{O}$ & -3.04470000 & 0.21520000 & 1.79640000 & \\
\hline $\mathrm{O}$ & -0.72400000 & -0.61490000 & -1.53700000 & \\
\hline $\mathrm{O}$ & -0.67410000 & 1.41390000 & 0.56680000 & \\
\hline $\mathrm{C}$ & -2.70620000 & -0.35200000 & 2.88020000 & \\
\hline $\mathrm{C}$ & 0.26550000 & -1.38970000 & -1.37770000 & \\
\hline $\mathrm{C}$ & 0.31350000 & 1.18800000 & 1.33190000 & \\
\hline $\mathrm{O}$ & -1.73180000 & -1.15800000 & 3.03850000 & \\
\hline $\mathrm{O}$ & 0.60950000 & -1.95520000 & -0.28530000 & \\
\hline $\mathrm{O}$ & 0.63190000 & 0.06370000 & 1.83750000 & \\
\hline $\mathrm{C}$ & 1.13860000 & -1.68440000 & -2.57390000 & \\
\hline $\mathrm{H}$ & 2.15350000 & -1.32780000 & -2.37520000 & \\
\hline $\mathrm{H}$ & 1.19350000 & -2.76560000 & -2.72720000 & \\
\hline $\mathrm{H}$ & 0.74720000 & -1.20070000 & -3.46830000 & \\
\hline $\mathrm{C}$ & -3.65370000 & -4.13750000 & -0.16400000 & \\
\hline $\mathrm{H}$ & -3.90630000 & -4.67730000 & 0.75180000 & \\
\hline $\mathrm{H}$ & -4.56130000 & -3.82310000 & -0.67860000 & \\
\hline $\mathrm{H}$ & -3.08940000 & -4.82080000 & -0.80620000 & \\
\hline $\mathrm{C}$ & -3.51080000 & -0.04000000 & 4.11960000 & \\
\hline $\mathrm{H}$ & -3.70940000 & -0.96000000 & 4.67360000 & \\
\hline $\mathrm{H}$ & -2.92220000 & 0.61800000 & 4.76680000 & \\
\hline $\mathrm{H}$ & -4.44540000 & 0.45660000 & 3.85890000 & \\
\hline $\mathrm{C}$ & 1.18650000 & 2.36040000 & 1.71220000 & \\
\hline
\end{tabular}




$\begin{array}{lrrr}\mathrm{H} & & & \\ \mathrm{H} & 2.75320000 & 2.84970000 & 2.59090000 \\ \mathrm{H} & 2.19060000 & 2.01970000 & 1.96780000 \\ \mathrm{C} & 1.22180000 & 3.08710000 & 0.89930000 \\ \mathrm{C} & 0.20200000 & -3.53930000 & 3.90350000 \\ \mathrm{C} & -1.16030000 & -3.89220000 & 3.94620000 \\ \mathrm{C} & 1.02550000 & -3.93760000 & 4.97960000 \\ \mathrm{H} & -1.68490000 & -4.57700000 & 5.04040000 \\ \mathrm{C} & -1.79780000 & -3.62690000 & 3.11490000 \\ \mathrm{H} & 0.50320000 & -4.62370000 & 6.06800000 \\ \mathrm{C} & 2.08540000 & -3.70730000 & 4.95110000 \\ \mathrm{H} & -0.85980000 & -4.94020000 & 6.10590000 \\ \mathrm{H} & -2.73980000 & -4.83280000 & 5.05770000 \\ \mathrm{H} & 1.15460000 & -4.91680000 & 6.88530000 \\ \mathrm{C} & -1.27000000 & -5.47660000 & 6.95600000 \\ \mathrm{C} & 0.75400000 & -2.84080000 & 2.72770000 \\ \mathrm{C} & 0.91400000 & -4.47300000 & 1.35690000 \\ \mathrm{H} & 1.86210000 & -5.16480000 & 2.08320000 \\ \mathrm{H} & 1.15700000 & -3.98330000 & 0.42190000 \\ \mathrm{H} & -0.13470000 & -4.70100000 & 1.48840000 \\ \mathrm{C} & 1.52720000 & -5.65380000 & 2.99370000 \\ \mathrm{C} & 3.28350000 & -5.20810000 & 1.84810000 \\ \mathrm{C} & 3.85650000 & -4.93270000 & 0.58680000 \\ \mathrm{C} & 4.13730000 & -5.49610000 & 2.93430000 \\ \mathrm{H} & 5.23590000 & -4.91280000 & 0.43100000 \\ \mathrm{C} & 3.21200000 & -4.74680000 & -0.26610000 \\ \mathrm{H} & 5.51830000 & -5.45960000 & 2.77820000 \\ \mathrm{C} & 3.70120000 & -5.72310000 & 3.90250000 \\ \mathrm{H} & 6.06950000 & -5.16460000 & 1.52770000 \\ \mathrm{H} & 5.66780000 & -4.70280000 & -0.54190000 \\ \mathrm{H} & 6.16590000 & -5.66220000 & 3.62470000 \\ \mathrm{Cl} & 7.14740000 & -5.14020000 & 1.40360000 \\ \mathrm{~N} & 1.14220000 & -0.80400000 & 5.13550000 \\ \mathrm{O} & 5.67490000 & 0.11370000 & 2.97160000 \\ \mathrm{O} & 6.47890000 & -0.02650000 & 2.04490000 \\ & 5.87310000 & 0.84270000 & 3.94860000\end{array}$




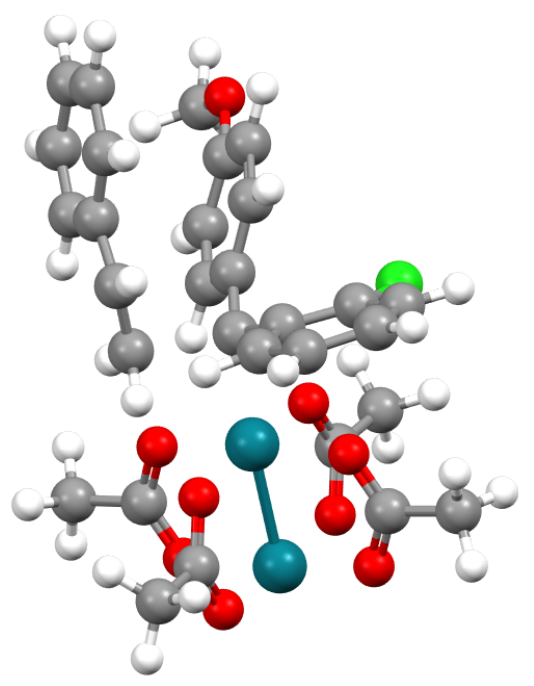

Entry SCF Energy (a. u.) Enthalpy (a. u.) Gibbs Free Energy (a. u.)

\begin{tabular}{|c|c|c|c|c|}
\hline \multirow{2}{*}{\multicolumn{2}{|c|}{$\begin{array}{l}\text { TS-3 for } \mathbf{8 g} \quad-2518 \\
\text { One imaginary frequency }\end{array}$}} & .46766058 & -2517.865021 & \multirow[t]{2}{*}{-2517.988174} \\
\hline & & \multirow[b]{2}{*}{-1.72380600} & \multirow[b]{2}{*}{1.40587100} & \\
\hline \multicolumn{2}{|c|}{$\mathrm{Rh} \quad-0.46956300$} & & & \\
\hline \multirow{2}{*}{$\begin{array}{l}\mathrm{O} \\
\mathrm{C}\end{array}$} & -1.82165100 & -3.21816300 & 0.86869300 & \\
\hline & -2.84415400 & -2.91256800 & 0.16380600 & \\
\hline $\mathrm{C}$ & 2.11128400 & -2.33631800 & 2.79118400 & \\
\hline \multirow{2}{*}{$\begin{array}{l}\mathrm{O} \\
\mathrm{C}\end{array}$} & -3.14078100 & -1.76263000 & -0.26854100 & \\
\hline & 2.35439700 & -1.36809200 & 3.79607800 & \\
\hline $\mathrm{C}$ & 3.11967700 & -2.44086400 & 1.80833800 & \\
\hline $\mathrm{C}$ & 3.53904200 & -0.63218900 & 3.85267000 & \\
\hline \multirow{2}{*}{$\begin{array}{l}\mathrm{C} \\
\mathrm{H}\end{array}$} & 4.29928200 & -1.71092500 & 1.85108800 & \\
\hline & 2.96071300 & -3.10312500 & 0.96975100 & \\
\hline $\mathrm{C}$ & 4.52594900 & -0.80666300 & 2.88955200 & \\
\hline $\mathrm{H}$ & 3.66562000 & 0.08966700 & 4.65157600 & \\
\hline \multirow{2}{*}{$\begin{array}{l}\mathrm{H} \\
\mathrm{Rh}\end{array}$} & 5.03932900 & -1.84753400 & 1.06870200 & \\
\hline & -1.89051000 & -0.15046000 & 0.14099400 & \\
\hline $\mathrm{O}$ & -2.92696200 & 0.21083500 & 1.89707000 & \\
\hline \multirow{2}{*}{$\begin{array}{l}\mathrm{O} \\
\mathrm{O}\end{array}$} & -0.74999200 & -0.60305100 & -1.53278300 & \\
\hline & -0.55953200 & 1.35392500 & 0.62788800 & \\
\hline $\mathrm{C}$ & -2.57378600 & -0.40452500 & 2.94791300 & \\
\hline $\mathrm{C}$ & 0.21696400 & -1.41257400 & -1.42758000 & \\
\hline \multirow{2}{*}{$\begin{array}{l}\mathrm{C} \\
\mathrm{O}\end{array}$} & 0.44410900 & 1.05907100 & 1.34626600 & \\
\hline & -1.63327100 & -1.25663800 & 3.04996300 & \\
\hline $\mathrm{O}$ & 0.57377100 & -2.03307500 & -0.37003200 & \\
\hline $\mathrm{O}$ & 0.74086300 & -0.09275800 & 1.79720300 & \\
\hline $\mathrm{C}$ & 1.05898200 & -1.66258200 & -2.65801000 & \\
\hline $\mathrm{H}$ & 2.00795600 & -1.12701400 & -2.55096900 & \\
\hline $\mathrm{H}$ & 1.28573900 & -2.72731400 & -2.74710300 & \\
\hline $\mathrm{H}$ & 0.54739600 & -1.30549100 & -3.55173800 & \\
\hline \multirow{2}{*}{$\begin{array}{l}\mathrm{C} \\
\mathrm{H}\end{array}$} & -3.76405000 & -4.05857800 & -0.19197500 & \\
\hline & -3.98618300 & -4.64701000 & 0.70167000 & \\
\hline $\begin{array}{l}\Pi \\
\mathrm{H}\end{array}$ & -4.68551800 & -3.68701700 & -0.63960600 & \\
\hline $\mathrm{H}$ & -3.25534000 & -4.71647400 & -0.90379700 & \\
\hline $\mathrm{C}$ & -3.31621000 & -0.09025100 & 4.22651400 & \\
\hline $\mathrm{H}$ & -3.44381900 & -1.00013900 & 4.81626100 & \\
\hline $\mathrm{H}$ & -2.71600500 & 0.60952900 & 4.81729600 & \\
\hline $\mathrm{H}$ & -4.28113100 & 0.36785800 & 4.00804800 & \\
\hline & & & S127 & \\
\hline
\end{tabular}




$\begin{array}{lrrr}\mathrm{C} & 1.37506700 & 2.18309800 & 1.73888100 \\ \mathrm{H} & 0.96632300 & 2.68258300 & 2.62365400 \\ \mathrm{H} & 2.36046100 & 1.78731500 & 1.98773700 \\ \mathrm{H} & 1.44154300 & 2.91742700 & 0.93438500 \\ \mathrm{C} & 0.21173500 & -3.66947000 & 3.82841100 \\ \mathrm{C} & -1.16402600 & -3.97374900 & 3.87115500 \\ \mathrm{C} & 0.98936700 & -4.05674500 & 4.94835400 \\ \mathrm{C} & -1.75181100 & -4.56506700 & 4.98411200 \\ \mathrm{H} & -1.77884000 & -3.72993600 & 3.01610100 \\ \mathrm{C} & 0.42310700 & -4.65401800 & 6.05624100 \\ \mathrm{H} & 2.05756500 & -3.87241200 & 4.93566700 \\ \mathrm{C} & -0.95897500 & -4.90727300 & 6.08732600 \\ \mathrm{H} & -2.81568800 & -4.76680800 & 4.97655500 \\ \mathrm{H} & 1.02420000 & -4.94830700 & 6.90925300 \\ \mathrm{H} & 5.44585200 & -0.23352100 & 2.94248100 \\ \mathrm{C} & 0.80157700 & -3.02875600 & 2.65338900 \\ \mathrm{C} & 0.89259600 & -4.49756100 & 1.27463400 \\ \mathrm{C} & 1.81947300 & -5.31987500 & 1.90456500 \\ \mathrm{H} & 1.19297900 & -3.92035900 & 0.40886900 \\ \mathrm{H} & -0.16040800 & -4.74891900 & 1.28196500 \\ \mathrm{H} & 2.87463000 & -5.11335000 & 1.74913700 \\ \mathrm{C} & 1.52702400 & -6.30174300 & 2.90710600 \\ \mathrm{C} & 2.57805900 & -6.77144700 & 3.72932500 \\ \mathrm{C} & 0.21381100 & -6.75202200 & 3.16929700 \\ \mathrm{C} & 2.32106800 & -7.61837000 & 4.79767800 \\ \mathrm{H} & 3.59089900 & -6.43297000 & 3.53044200 \\ \mathrm{C} & -0.03931800 & -7.59417900 & 4.24252300 \\ \mathrm{H} & -0.60145300 & -6.43026000 & 2.53404600 \\ \mathrm{C} & 1.00730300 & -8.01829600 & 5.06680700 \\ \mathrm{H} & 3.13396500 & -7.95668900 & 5.43211100 \\ \mathrm{H} & -1.05507200 & -7.91595600 & 4.44756400 \\ \mathrm{H} & 0.80141500 & -8.66797400 & 5.91176600 \\ \mathrm{Cl} & 1.13953000 & -0.92053600 & 4.99034600 \\ \mathrm{O} & -1.42554900 & -5.49825300 & 7.21647800 \\ \mathrm{C} & -2.81550300 & -5.79239000 & 7.29371700 \\ \mathrm{H} & -2.97223900 & -6.25131600 & 8.27009900 \\ \mathrm{H} & -3.42145900 & -4.88230600 & 7.21680800 \\ \mathrm{H} & -3.11859100 & -6.49452700 & 6.50777500\end{array}$




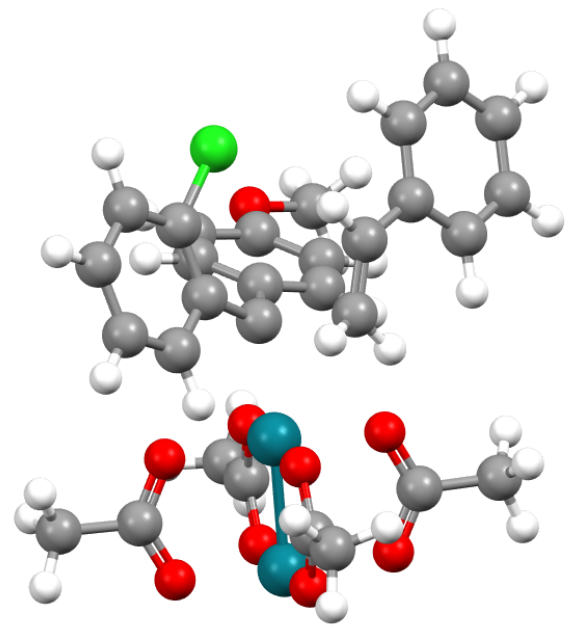

Entry SCF Energy (a. u.) Enthalpy (a. u.) $\quad$ Gibbs Free Energy (a. u.)

\begin{tabular}{|c|c|c|c|c|}
\hline \multirow{2}{*}{\multicolumn{2}{|c|}{$\begin{array}{l}\text { TS-4 for } 8 g \\
\text { One imaginary frequency }\end{array}$}} & \multirow[b]{3}{*}{-1.74564800} & \multirow{3}{*}{$\begin{array}{l}-2517.854127 \\
1.42966900\end{array}$} & \multirow[t]{2}{*}{-2517.979489} \\
\hline & & & & \\
\hline $\mathrm{Rh}$ & -0.44919400 & & & \\
\hline $\mathrm{O}$ & -1.74757600 & -3.31616200 & 1.00688000 & \\
\hline $\mathrm{C}$ & -2.72923300 & -3.11887400 & 0.21220800 & \\
\hline $\mathrm{C}$ & 2.01993600 & -2.14108100 & 3.02587700 & \\
\hline $\mathrm{O}$ & -3.01880000 & -2.03296700 & -0.36737300 & \\
\hline $\mathrm{C}$ & 2.25966500 & -1.41192500 & 4.21505900 & \\
\hline $\mathrm{C}$ & 2.88178600 & -1.89377300 & 1.94091900 & \\
\hline $\mathrm{C}$ & 3.31153200 & -0.52035900 & 4.31511800 & \\
\hline $\mathrm{H}$ & 1.58737800 & -1.52731600 & 5.05716600 & \\
\hline $\mathrm{C}$ & 3.97557200 & -1.04465000 & 2.04487800 & \\
\hline $\mathrm{H}$ & 2.66442700 & -2.34936200 & 0.98780000 & \\
\hline $\mathrm{C}$ & 4.19711800 & -0.34668400 & 3.23939000 & \\
\hline $\mathrm{H}$ & 3.47931200 & 0.04903300 & 5.22306500 & \\
\hline $\mathrm{H}$ & 4.62543700 & -0.91295300 & 1.18878300 & \\
\hline $\mathrm{Rh}$ & -1.83690500 & -0.34995000 & -0.06343200 & \\
\hline $\mathrm{O}$ & -2.97490600 & 0.15817000 & 1.58744000 & \\
\hline $\mathrm{O}$ & -0.60451600 & -0.92852900 & -1.62772800 & \\
\hline $\mathrm{O}$ & -0.56880900 & 1.23627300 & 0.32366600 & \\
\hline $\mathrm{C}$ & -2.63160400 & -0.28340200 & 2.72420900 & \\
\hline $\mathrm{C}$ & 0.35634300 & -1.71764100 & -1.40130700 & \\
\hline $\mathrm{C}$ & 0.39952400 & 1.04922700 & 1.12332400 & \\
\hline $\mathrm{O}$ & -1.65992800 & -1.07119300 & 2.97126100 & \\
\hline $\mathrm{O}$ & 0.66807000 & -2.22398500 & -0.27040100 & \\
\hline $\mathrm{O}$ & 0.69274500 & -0.03597800 & 1.71739800 & \\
\hline $\mathrm{C}$ & 1.22809700 & -2.12570300 & -2.56689100 & \\
\hline $\mathrm{H}$ & 1.09229700 & -3.19451900 & -2.75951100 & \\
\hline $\mathrm{H}$ & 0.96742600 & -1.55905600 & -3.46037700 & \\
\hline $\mathrm{H}$ & 2.27917000 & -1.96618600 & -2.31211200 & \\
\hline $\mathrm{C}$ & -3.60573700 & -4.31925800 & -0.06323800 & \\
\hline $\mathrm{H}$ & -3.04969300 & -5.03255300 & -0.67996300 & \\
\hline $\mathrm{H}$ & -3.85623100 & -4.81907300 & 0.87546600 & \\
\hline $\mathrm{H}$ & -4.51265500 & -4.01977300 & -0.58809400 & \\
\hline $\mathrm{C}$ & -3.42606000 & 0.18192800 & 3.92276500 & \\
\hline $\mathrm{H}$ & -3.47929600 & -0.61152100 & 4.67023000 & \\
\hline $\mathrm{H}$ & -2.91247000 & 1.03794800 & 4.37323300 & \\
\hline $\mathrm{H}$ & -4.42519900 & 0.49588500 & 3.61910400 & \\
\hline
\end{tabular}




\begin{tabular}{lrrr}
$\mathrm{C}$ & 1.28737700 & 2.23294700 & 1.43300800 \\
$\mathrm{H}$ & 0.88062800 & 2.75927100 & 2.30304900 \\
$\mathrm{H}$ & 2.29372200 & 1.89169900 & 1.68089000 \\
$\mathrm{H}$ & 1.30657600 & 2.92533100 & 0.59023500 \\
$\mathrm{C}$ & 0.17125100 & -3.55028600 & 4.06644200 \\
$\mathrm{C}$ & -1.23731100 & -3.66930200 & 4.08738800 \\
$\mathrm{C}$ & 0.83849900 & -4.10238900 & 5.19125000 \\
$\mathrm{C}$ & -1.93575300 & -4.19948100 & 5.16482200 \\
$\mathrm{H}$ & -1.78118800 & -3.32561700 & 3.22269700 \\
$\mathrm{C}$ & 0.14852500 & -4.63444000 & 6.28072900 \\
$\mathrm{C}$ & -1.24311600 & -4.66838500 & 6.27946400 \\
$\mathrm{H}$ & -3.01928900 & -4.25008500 & 5.12840300 \\
$\mathrm{H}$ & 0.71088300 & -5.03516600 & 7.11606700 \\
$\mathrm{H}$ & -1.77193800 & -5.08183800 & 7.13245700 \\
$\mathrm{C}$ & 0.79925500 & -2.92380400 & 2.86756300 \\
$\mathrm{C}$ & 0.93345500 & -4.55675700 & 1.58940100 \\
$\mathrm{C}$ & 2.01345900 & -5.20676000 & 2.15914200 \\
$\mathrm{H}$ & 1.03207700 & -4.06267200 & 0.63241000 \\
$\mathrm{H}$ & -0.07000200 & -4.88529500 & 1.82403900 \\
$\mathrm{H}$ & 1.84464800 & -5.79214400 & 3.05768000 \\
$\mathrm{C}$ & 3.38366000 & -5.11581700 & 1.71433200 \\
$\mathrm{C}$ & 3.73660500 & -4.57712700 & 0.45641200 \\
$\mathrm{C}$ & 4.41796300 & -5.58220700 & 2.55395100 \\
$\mathrm{C}$ & 5.06818600 & -4.48877300 & 0.07067500 \\
$\mathrm{H}$ & 2.95966000 & -4.24009300 & -0.22095500 \\
$\mathrm{C}$ & 5.74934800 & -5.48863700 & 2.16738300 \\
$\mathrm{H}$ & 4.15904200 & -6.00208200 & 3.52021500 \\
$\mathrm{C}$ & 6.08001900 & -4.93581600 & 0.92636700 \\
$\mathrm{H}$ & 5.32151900 & -4.07407600 & -0.90007900 \\
$\mathrm{H}$ & 6.53169900 & -5.84251400 & 2.83133100 \\
$\mathrm{H}$ & 7.11999500 & -4.86156100 & 0.62385400 \\
$\mathrm{Cl}$ & 2.59294100 & -4.26791100 & 5.28138500 \\
$\mathrm{O}$ & 5.22174100 & 0.52450700 & 3.44685100 \\
$\mathrm{C}$ & 6.13347100 & 0.74645500 & 2.37934100 \\
$\mathrm{H}$ & 6.86693300 & 1.46184600 & 2.75248800 \\
$\mathrm{H}$ & 6.64362200 & -0.17996600 & 2.08880900 \\
$\mathrm{H}$ & 5.62802800 & 1.16792800 & 1.50195800 \\
& & & \\
\hline & & & \\
& & &
\end{tabular}




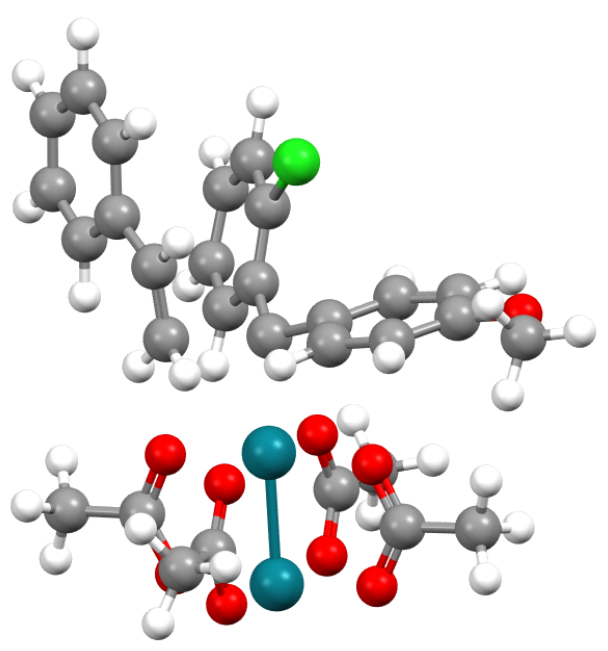

Entry SCF Energy (a. u.) Enthalpy (a. u.) $\quad$ Gibbs Free Energy (a.

\begin{tabular}{|c|c|c|c|c|}
\hline & & & & u.) \\
\hline TS-5 for $8 g$ & -2518. & .46144224 & -2517.859065 & -2517.983642 \\
\hline One imagin & ary frequency & & & \\
\hline $\mathrm{Rh}$ & -0.34120000 & -1.72360000 & 1.44440000 & \\
\hline $\mathrm{O}$ & -1.70140000 & -3.26390000 & 1.07910000 & \\
\hline $\mathrm{C}$ & -2.77180000 & -3.01200000 & 0.42440000 & \\
\hline $\mathrm{C}$ & 2.19710000 & -2.14690000 & 2.88870000 & \\
\hline $\mathrm{O}$ & -3.10450000 & -1.89680000 & -0.06610000 & \\
\hline $\mathrm{C}$ & 2.36550000 & -1.33500000 & 4.03630000 & \\
\hline $\mathrm{C}$ & 3.12700000 & -1.98630000 & 1.84870000 & \\
\hline $\mathrm{C}$ & 3.39070000 & -0.41190000 & 4.12550000 & \\
\hline $\mathrm{C}$ & 4.18810000 & -1.09290000 & 1.94340000 & \\
\hline $\mathrm{H}$ & 3.02010000 & -2.56390000 & 0.94010000 & \\
\hline $\mathrm{C}$ & 4.31830000 & -0.28490000 & 3.08140000 & \\
\hline $\mathrm{H}$ & 3.49910000 & 0.22270000 & 4.99870000 & \\
\hline $\mathrm{H}$ & 4.89290000 & -1.02020000 & 1.12430000 & \\
\hline $\mathrm{Rh}$ & -1.81600000 & -0.26990000 & 0.09120000 & \\
\hline $\mathrm{O}$ & -2.74540000 & 0.30060000 & 1.85270000 & \\
\hline $\mathrm{O}$ & -0.78720000 & -0.92480000 & -1.58530000 & \\
\hline $\mathrm{O}$ & -0.43780000 & 1.25470000 & 0.32640000 & \\
\hline $\mathrm{C}$ & -2.35000000 & -0.20550000 & 2.94410000 & \\
\hline $\mathrm{C}$ & 0.20630000 & -1.69450000 & -1.43820000 & \\
\hline $\mathrm{C}$ & 0.57190000 & 1.04020000 & 1.06440000 & \\
\hline $\mathrm{O}$ & -1.42090000 & -1.06770000 & 3.09090000 & \\
\hline $\mathrm{O}$ & 0.63520000 & -2.17690000 & -0.33650000 & \\
\hline $\mathrm{O}$ & 0.85830000 & -0.04610000 & 1.66030000 & \\
\hline $\mathrm{C}$ & 0.99200000 & -2.06680000 & -2.67490000 & \\
\hline $\mathrm{H}$ & 0.40000000 & -1.88480000 & -3.57200000 & \\
\hline $\mathrm{H}$ & 1.89580000 & -1.44990000 & -2.71670000 & \\
\hline $\mathrm{H}$ & 1.30150000 & -3.11270000 & -2.62540000 & \\
\hline $\mathrm{C}$ & -3.70310000 & -4.18400000 & 0.21450000 & \\
\hline $\mathrm{H}$ & -4.66340000 & -3.84670000 & -0.17500000 & \\
\hline $\mathrm{H}$ & -3.24710000 & -4.87680000 & -0.50010000 & \\
\hline $\mathrm{H}$ & -3.84120000 & -4.72230000 & 1.15530000 & \\
\hline $\mathrm{C}$ & -3.01720000 & 0.26650000 & 4.21570000 & \\
\hline $\mathrm{H}$ & -3.09660000 & -0.55790000 & 4.92670000 & \\
\hline $\mathrm{H}$ & -2.39600000 & 1.04550000 & 4.67020000 & \\
\hline $\mathrm{H}$ & -4.00020000 & 0.68520000 & 3.99810000 & \\
\hline $\mathrm{C}$ & 1.52670000 & 2.19070000 & 1.28880000 & \\
\hline
\end{tabular}




$\begin{array}{lrrr}\mathrm{H} & 1.62340000 & 2.78170000 & 0.37610000 \\ \mathrm{H} & 1.11830000 & 2.84040000 & 2.07000000 \\ \mathrm{H} & 2.49800000 & 1.81810000 & 1.61600000 \\ \mathrm{C} & 0.40380000 & -3.58270000 & 3.94610000 \\ \mathrm{C} & -0.99660000 & -3.74790000 & 4.05190000 \\ \mathrm{C} & 1.16530000 & -4.17040000 & 4.99540000 \\ \mathrm{C} & -1.60320000 & -4.36720000 & 5.13760000 \\ \mathrm{H} & -1.60660000 & -3.36940000 & 3.24790000 \\ \mathrm{C} & 0.56610000 & -4.80130000 & 6.08290000 \\ \mathrm{C} & -0.82130000 & -4.88850000 & 6.16560000 \\ \mathrm{H} & -2.68450000 & -4.45320000 & 5.17060000 \\ \mathrm{H} & 1.19390000 & -5.23860000 & 6.85010000 \\ \mathrm{C} & 0.94740000 & -2.89520000 & 2.75620000 \\ \mathrm{C} & 1.13840000 & -4.50660000 & 1.32260000 \\ \mathrm{C} & 1.87490000 & -5.42320000 & 2.03260000 \\ \mathrm{H} & 1.60630000 & -3.89530000 & 0.56530000 \\ \mathrm{H} & 0.06900000 & -4.62240000 & 1.20170000 \\ \mathrm{H} & 2.95840000 & -5.33240000 & 2.02730000 \\ \mathrm{C} & 1.32990000 & -6.45550000 & 2.88350000 \\ \mathrm{C} & 2.20660000 & -7.24480000 & 3.65900000 \\ \mathrm{C} & -0.05890000 & -6.67940000 & 3.00560000 \\ \mathrm{C} & 1.71720000 & -8.19840000 & 4.54160000 \\ \mathrm{H} & 3.27570000 & -7.07760000 & 3.57490000 \\ \mathrm{C} & -0.54540000 & -7.62930000 & 3.89400000 \\ \mathrm{H} & -0.75400000 & -6.09510000 & 2.41550000 \\ \mathrm{C} & 0.33710000 & -8.38660000 & 4.66980000 \\ \mathrm{H} & 2.40540000 & -8.78890000 & 5.13820000 \\ \mathrm{H} & -1.61630000 & -7.77700000 & 3.98960000 \\ \mathrm{H} & -0.04810000 & -9.12430000 & 5.36710000 \\ \mathrm{Cl} & 2.92590000 & -4.23410000 & 4.98310000 \\ \mathrm{H} & 1.65030000 & -1.41130000 & 4.84750000 \\ \mathrm{H} & -1.27790000 & -5.38090000 & 7.01830000 \\ \mathrm{O} & 5.29830000 & 0.64260000 & 3.26390000 \\ \mathrm{C} & 6.23240000 & 0.84210000 & 2.21160000 \\ \mathrm{H} & 6.90560000 & 1.62990000 & 2.55110000 \\ \mathrm{H} & 6.81110000 & -0.06740000 & 2.00980000 \\ \mathrm{H} & 5.73340000 & 1.16250000 & 1.28890000\end{array}$




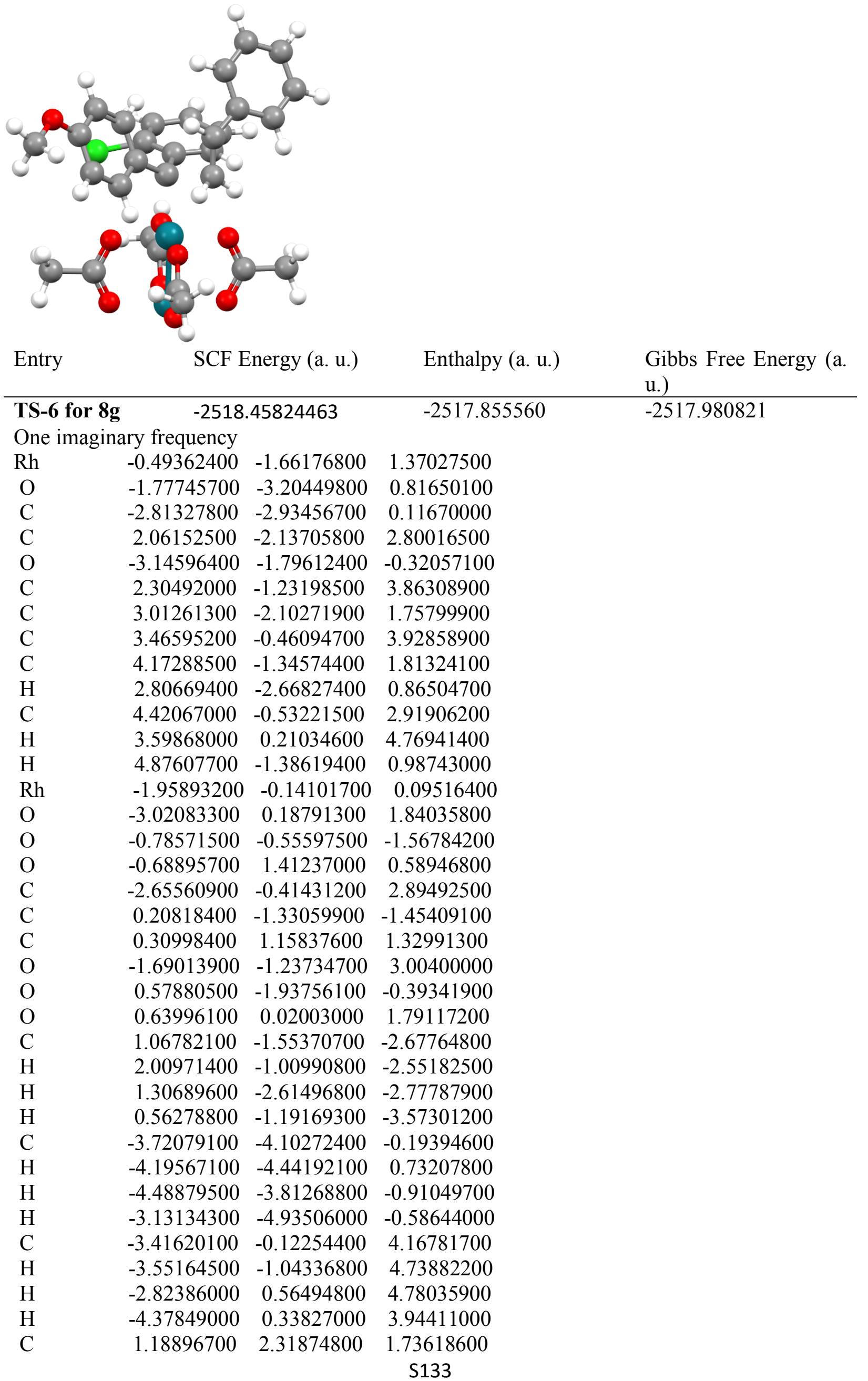




\begin{tabular}{lrrr}
$\mathrm{H}$ & 0.74439200 & 2.80459500 & 2.61123300 \\
$\mathrm{H}$ & 2.18430700 & 1.96302000 & 2.00497100 \\
$\mathrm{H}$ & 1.24176500 & 3.05282000 & 0.93045400 \\
$\mathrm{C}$ & 0.22399500 & -3.58189100 & 3.82345400 \\
$\mathrm{C}$ & -1.12875000 & -3.95736500 & 3.83980200 \\
$\mathrm{C}$ & 1.01505100 & -3.96613500 & 4.93111800 \\
$\mathrm{C}$ & -1.68955400 & -4.63735900 & 4.91898500 \\
$\mathrm{H}$ & -1.75136700 & -3.71287000 & 2.99056200 \\
$\mathrm{C}$ & 0.47828900 & -4.64831400 & 6.00575400 \\
$\mathrm{H}$ & 2.07310800 & -3.72523500 & 4.93287200 \\
$\mathrm{C}$ & -0.88883200 & -4.98069100 & 6.01451900 \\
$\mathrm{H}$ & -2.74080300 & -4.89611300 & 4.89282600 \\
$\mathrm{H}$ & 1.08854100 & -4.93829100 & 6.85445700 \\
$\mathrm{H}$ & 5.32480300 & 0.06450900 & 2.98424300 \\
$\mathrm{C}$ & 0.80725900 & -2.90919600 & 2.64506600 \\
$\mathrm{C}$ & 0.95932400 & -4.38361700 & 1.30162200 \\
$\mathrm{C}$ & 1.86787600 & -5.14747000 & 2.02779600 \\
$\mathrm{H}$ & 1.25266800 & -3.89221500 & 0.38178600 \\
$\mathrm{H}$ & -0.09035100 & -4.64394900 & 1.33994600 \\
$\mathrm{H}$ & 1.48285400 & -5.66280000 & 2.90278100 \\
$\mathrm{C}$ & 3.29466000 & -5.21537300 & 1.85856200 \\
$\mathrm{C}$ & 3.93745900 & -4.90395100 & 0.64002200 \\
$\mathrm{C}$ & 4.08975700 & -5.58826700 & 2.96431400 \\
$\mathrm{C}$ & 5.32218600 & -4.93709400 & 0.54402300 \\
$\mathrm{H}$ & 3.33965700 & -4.65334100 & -0.23013000 \\
$\mathrm{C}$ & 5.47602300 & -5.60632900 & 2.86900700 \\
$\mathrm{H}$ & 3.60090700 & -5.83853400 & 3.90132200 \\
$\mathrm{C}$ & 6.09564300 & -5.27746400 & 1.65958800 \\
$\mathrm{H}$ & 5.80476100 & -4.69990000 & -0.39874300 \\
$\mathrm{H}$ & 6.07592800 & -5.87619600 & 3.73219300 \\
$\mathrm{H}$ & 7.17827800 & -5.29573800 & 1.58264200 \\
$\mathrm{Cl}$ & 1.10212000 & -0.87425300 & 5.10111700 \\
$\mathrm{O}$ & -1.32816600 & -5.64231900 & 7.11603100 \\
$\mathrm{C}$ & -2.70467800 & -5.99753700 & 7.17870500 \\
$\mathrm{H}$ & -2.84266300 & -6.49852400 & 8.13710900 \\
$\mathrm{H}$ & -3.34771700 & -5.11079300 & 7.13434300 \\
$\mathrm{H}$ & -2.97829700 & -6.68207000 & 6.36707900 \\
& & & \\
\hline & & & \\
& & &
\end{tabular}




\section{X-ray Crystallographic Data}

\section{Cyclopropane-1,1,2-triyltribenzene (11a)}

\section{Crystal Data and Experimental}

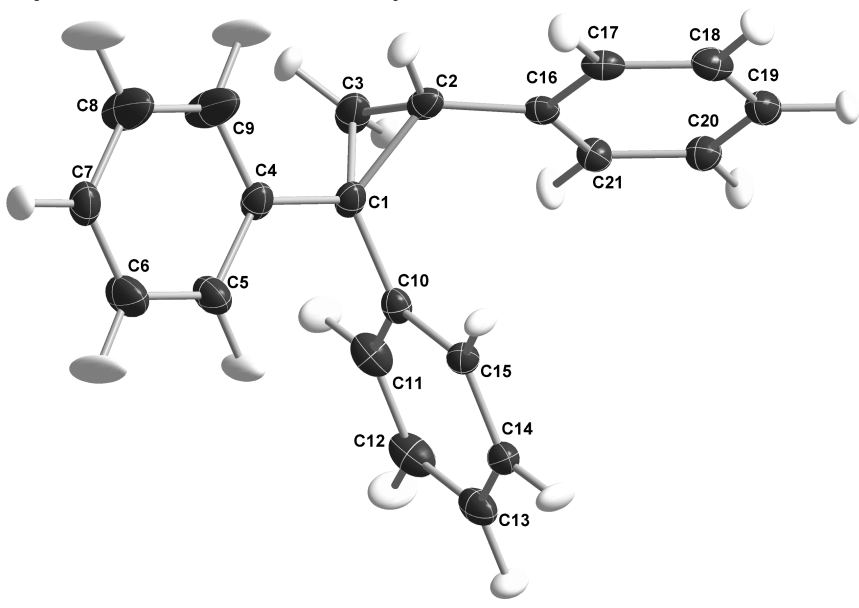

Experimental. Single colorless needle-shaped crystals of cyclopropane-1,1,2-triyltribenzene (11a) were chosen from the sample as supplied. A suitable crystal $0.53 \times 0.21 \times 0.09 \mathrm{~mm}^{3}$ was selected and mounted on a loop with paratone oil on an XtaLAB Synergy-S diffractometer. The crystal was kept at a steady $T=100.0(1) \mathrm{K}$ during data collection. The structure was solved with the SheIXT solution program using dual methods and by using Olex2 as the graphical interface. ${ }^{18}$ The model was refined with version of olex2.refine 1.3-alpha using Levenberg-Marquardt minimisation. ${ }^{19}$

Crystal Data. $\mathrm{C}_{21} \mathrm{H}_{18}, M_{r}=270.377$, orthorhombic, $P 2{ }_{1} 2{ }_{1} 2_{1}$ (No. 19), $a=5.76988(7) \AA, \quad b=11.91662(14) \AA, \quad c=$ 21.9056(2) $, \quad \alpha=\beta=\gamma=90^{\circ}, \quad V=1506.17(3) \AA^{3}, \quad T=$ 100.00(10) $\mathrm{K}, Z=4, Z^{\prime}=1, \mu\left(\mathrm{Cu} \mathrm{K}_{\alpha}\right)=0.505,16056$ reflections measured, 2565 unique $\left(R_{\text {int }}=0.0307\right)$ which were used in all calculations. The final $w R_{2}$ was 0.0667 (all data) and $R_{1}$ was $0.0267(I \geq \sigma(I))$. 


\begin{tabular}{|c|c|}
\hline Compound & $\begin{array}{l}\text { cyclopropane-1 } \\
\text { triyltribenzene } \\
\text { (11a) }\end{array}$ \\
\hline Formula & $\mathrm{C}_{21} \mathrm{H}_{18}$ \\
\hline$D_{\text {calc. }} / \mathrm{g} \mathrm{cm}^{-3}$ & 1.192 \\
\hline$\mu / \mathrm{mm}^{-1}$ & 0.505 \\
\hline Formula Weight & 270.377 \\
\hline Color & colorless \\
\hline Shape & needle \\
\hline Size $/ \mathrm{mm}^{3}$ & $0.53 \times 0.21 \times 0.09$ \\
\hline$T / \mathrm{K}$ & $100.00(10)$ \\
\hline Crystal System & orthorhombic \\
\hline Flack Parameter & $-0.14(4)$ \\
\hline Hooft Parameter & $0.0(4)$ \\
\hline Space Group & $P 2{ }_{1} 2_{1} 2_{1}$ \\
\hline$a / \AA$ & $5.76988(7)$ \\
\hline$b / \AA$ & $11.91662(14)$ \\
\hline$c / \AA ̊$ & $21.9056(2)$ \\
\hline$\left.\alpha\right|^{\circ}$ & 90 \\
\hline$\beta l^{\circ}$ & 90 \\
\hline$\gamma I^{\circ}$ & 90 \\
\hline$V / \AA^{3}$ & $1506.17(3)$ \\
\hline$Z$ & 4 \\
\hline$Z^{\prime}$ & 1 \\
\hline Wavelength/Å & 1.54184 \\
\hline Radiation type & $\mathrm{Cu} \mathrm{K} \alpha$ \\
\hline$\Theta_{\min } I^{\circ}$ & 4.04 \\
\hline$\Theta_{\max } /^{\circ}$ & 65.08 \\
\hline Measured Refl. & 16056 \\
\hline Independent Refl. & 2565 \\
\hline $\begin{array}{l}\text { Reflections with } \\
\mathrm{I} \geq \sigma(\mathrm{I})\end{array}$ & 2523 \\
\hline$R_{\text {int }}$ & 0.0307 \\
\hline Parameters & 354 \\
\hline Restraints & 264 \\
\hline Largest Peak & 0.2629 \\
\hline Deepest Hole & -0.2242 \\
\hline GooF & 1.0842 \\
\hline$w R_{2}$ (all data) & 0.0667 \\
\hline$w R_{2}$ & 0.0665 \\
\hline$R_{1}$ (all data) & 0.0272 \\
\hline$R_{1}$ & 0.0262 \\
\hline
\end{tabular}


Structure Quality Indicators

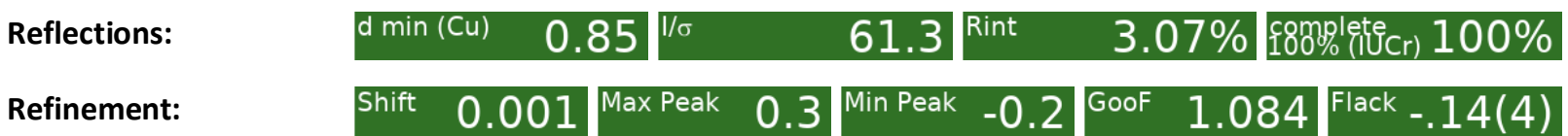

Experimental Extended. A colorless needle-shaped crystal with dimensions $0.53 \times 0.21 \times 0.09 \mathrm{~mm}^{3}$ was mounted on a loop with paratone oil. Data were collected using an XtaLAB Synergy, Dualflex, HyPix diffractometer equipped with an Oxford Cryosystems low-temperature device operating at $T=100.0(1) \mathrm{K}$.

Data were measured using $\omega$ scans of $0.5^{\circ}$ per frame for 1.0/0.2/6.6/4.0 s using $\mathrm{Cu} \mathrm{K}_{\alpha}$ radiation. The total number of runs and images was based on the strategy calculation from the program CrysAlisPro (Rigaku, V1.171.40.68a, 2019). The maximum resolution that was achieved was $\Theta=65.08^{\circ}(0.85 \AA)$.

The diffraction pattern was indexed and the total number of runs and images was based on the strategy calculation from the program CrysAlisPro (Rigaku, V1.171.40.68a, 2019). The unit cell was refined using CrysAlisPro (Rigaku, V1.171.40.68a, 2019) on 14976 reflections, 93\% of the observed reflections.

Data reduction, scaling and absorption corrections were performed using CrysAlisPro (Rigaku, V1.171.40.68a, 2019). The final completeness is $100.00 \%$ out to $65.08^{\circ}$ in $\Theta$. A numerical absorption correction based on Gaussian integration over a multifaceted crystal model was performed using CrysAlisPro 1.171.40.68a (Rigaku Oxford Diffraction, 2019). An empirical absorption correction using spherical harmonicsas implemented in SCALE3 ABSPACK was also used. The absorption coefficient $\mu$ of this material is $0.505 \mathrm{~mm}^{-1}$ at this wavelength $(\lambda=1.542 \AA)$ and the minimum and maximum transmissions are 0.488 and 1.000 .

The structure was solved and the space group $P 2{ }_{1} 2_{1} 2_{1}$ (\# 19) determined by the SHELXT structure solution program using Intrinsic Phasing and refined by Levenberg-Marquardt using version of olex2.refine 1.3-alpha. ${ }^{19}$ All atoms were refined anisotropically. Hydrogen atom positions were located and refined freely.

_refine_special_details: Refinement using NoSpherA2, an implementation of NOn-SPHERical Atom-formfactors in Olex2. ${ }^{20}$ NoSpherA2 makes use of tailor-made aspherical atomic form factors calculatedon-the-fly from a Hirshfeld-partitioned electron density (ED) - not fromspherical-atom form factors. The ED is calculated from a gaussian basis set single determinant SCFwavefunction - either Hartree-Fock or B3LYP - for a fragment of the crystal embedded inan electrostatic crystal field.The following options were used: SOFTWARE: Tonto METHOD: rks BASIS SET: STO-3G CHARGE: 0 MULTIPLICITY: 1 DATE: 2020-01-15_18-03-08 CLUSTER RADIUS: 0

There is a single molecule in the asymmetric unit, which is represented by the reported sum formula. In other words: $Z$ is 4 and $Z^{\prime}$ is 1.

The Flack parameter was refined to -0.14(4). Determination of absolute structure using Bayesian statistics on Bijvoet differences using the Olex2 results in 0.0(4). Note: The Flack parameter is used to determine chirality of the crystal studied, and the value near 0 means that the absolute structure is correct. 


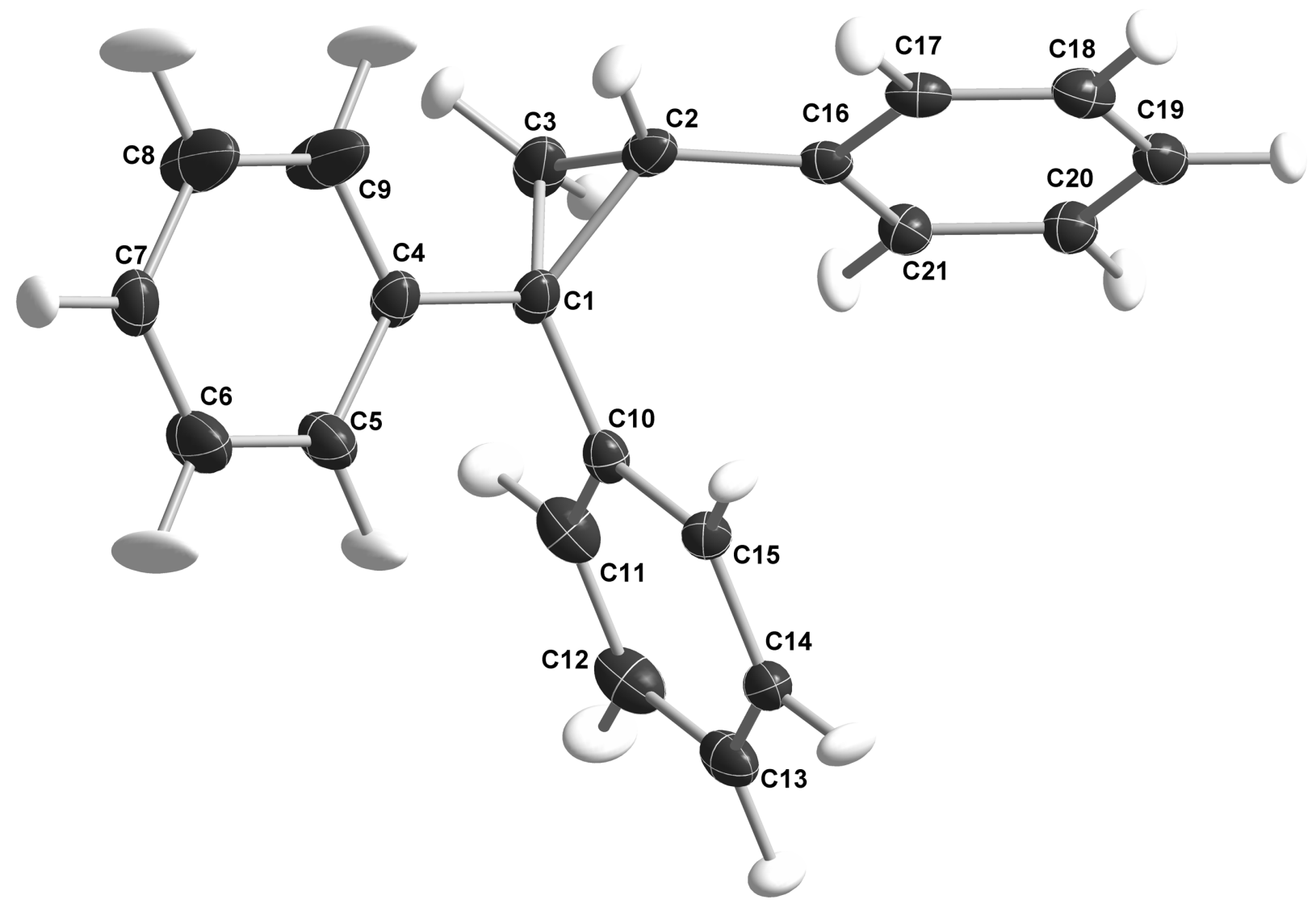

Figure S1:

Table S3: Fractional Atomic Coordinates $\left(\times 10^{4}\right)$ and Equivalent Isotropic Displacement Parameters $\left(\AA^{2} \times 10^{3}\right)$ for 11 . $U_{e q}$ is defined as $1 / 3$ of the trace of the orthogonalised $U_{i j}$.

\begin{tabular}{llccc}
\hline Atom & \multicolumn{1}{c}{$\mathbf{x}$} & $\mathbf{y}$ & $\mathbf{z}$ & $\boldsymbol{U}_{\text {eq }}$ \\
\hline C1 & $-7161(2)$ & $-4972.6(11)$ & $-3540.3(5)$ & $21.0(3)$ \\
C2 & $-6936(2)$ & $-3959.0(10)$ & $-3974.7(6)$ & $22.8(3)$ \\
C3 & $-8971(3)$ & $-4061.9(12)$ & $-3555.6(6)$ & $28.1(3)$ \\
C10 & $-7773(2)$ & $-6098.0(10)$ & $-3808.6(5)$ & $18.5(3)$ \\
C11 & $-9870(2)$ & $-6614.5(12)$ & $-3664.3(6)$ & $26.6(3)$ \\
C12 & $-10403(2)$ & $-7673.6(12)$ & $-3899.3(7)$ & $30.9(3)$ \\
C13 & $-8853(2)$ & $-8223.3(11)$ & $-4279.7(6)$ & $27.8(3)$ \\
C14 & $-6760(2)$ & $-7710.9(10)$ & $-4426.6(6)$ & $23.3(3)$ \\
C15 & $-6229(2)$ & $-6653.7(10)$ & $-4193.3(5)$ & $18.9(3)$ \\
C16 & $-7138.4(19)$ & $-4028.6(9)$ & $-4650.0(5)$ & $17.9(3)$ \\
C17 & $-5371(2)$ & $-3552.0(10)$ & $-5005.3(6)$ & $22.1(3)$ \\
C18 & $-5501(2)$ & $-3540.8(10)$ & $-5637.3(6)$ & $24.8(3)$ \\
C19 & $-7404(2)$ & $-4003.0(11)$ & $-5932.6(6)$ & $24.0(3)$ \\
C20 & $-9161(2)$ & $-4485.5(10)$ & $-5586.1(5)$ & $21.7(3)$ \\
C21 & $-9035(2)$ & $-4499.9(10)$ & $-4954.3(5)$ & $20.2(3)$ \\
C4 & $-5542.6(19)$ & $-4989.3(11)$ & $-2999.7(5)$ & $23.3(3)$ \\
C5 & $-4803(2)$ & $-5985.9(12)$ & $-2741.2(5)$ & $29.0(3)$ \\
C6 & $-3268(2)$ & $-5994.7(12)$ & $-2248.6(6)$ & $37.1(4)$ \\
C7 & $-2441(2)$ & $-5015.7(12)$ & $-2002.8(5)$ & $28.4(3)$ \\
C8 & $-3147(3)$ & $-4017.2(14)$ & $-2251.8(7)$ & $58.1(5)$ \\
C9 & $-4674(3)$ & $-4003.9(13)$ & $-2741.6(6)$ & $61.8(6)$
\end{tabular}

Table S4: Anisotropic Displacement Parameters $\left(\times 10^{4}\right)$ cyclopropane-1,1,2-triyltribenzene. The anisotropic 
displacement factor exponent takes the form: $-2 \pi^{2}\left[h^{2} a^{* 2} \times U_{11}+\ldots+2 h k a^{*} \times b^{*} \times U_{12}\right]$

\begin{tabular}{|c|c|c|c|c|c|c|}
\hline Atom & $U_{11}$ & $U_{22}$ & $U_{33}$ & $U_{23}$ & $U_{13}$ & $U_{12}$ \\
\hline$\overline{C 1}$ & $23.4(6)$ & $22.2(6)$ & $17.2(5)$ & $7.6(5)$ & $-1.1(4)$ & $-2.8(4)$ \\
\hline $\mathrm{C} 2$ & $27.0(6)$ & $17.9(6)$ & $23.5(6)$ & $6.3(5)$ & $-7.7(5)$ & $-3.1(5)$ \\
\hline C3 & $33.3(7)$ & $28.9(7)$ & $22.1(6)$ & $14.5(6)$ & $-3.0(6)$ & $-2.5(5)$ \\
\hline C10 & $17.1(6)$ & $22.0(6)$ & $16.6(5)$ & $3.4(5)$ & $-0.7(4)$ & $2.5(5)$ \\
\hline C11 & $15.8(6)$ & $33.1(7)$ & $30.9(7)$ & $1.6(5)$ & $0.0(5)$ & $8.8(5)$ \\
\hline C12 & $20.6(7)$ & $33.7(7)$ & $38.5(7)$ & $-5.2(5)$ & $-8.3(5)$ & $12.1(5)$ \\
\hline C13 & $32.7(7)$ & $24.4(6)$ & $26.3(6)$ & $-5.7(5)$ & $-12.2(5)$ & 7.1(5) \\
\hline C14 & $32.6(7)$ & $18.9(6)$ & $18.3(6)$ & $0.3(5)$ & $-3.8(5)$ & $0.8(4)$ \\
\hline C15 & $20.2(6)$ & $17.7(6)$ & $18.9(6)$ & $1.6(5)$ & $0.1(5)$ & $0.3(4)$ \\
\hline C16 & $17.5(5)$ & $14.1(5)$ & $22.2(6)$ & $2.3(4)$ & $-3.0(4)$ & $0.7(4)$ \\
\hline C17 & $17.5(6)$ & $15.6(6)$ & $33.3(7)$ & $0.2(5)$ & $-0.6(5)$ & $0.7(5)$ \\
\hline $\mathrm{C} 18$ & $23.8(6)$ & $19.4(6)$ & $31.0(7)$ & $0.5(5)$ & $5.4(5)$ & $2.8(5)$ \\
\hline C19 & $27.6(6)$ & $20.5(6)$ & $23.8(6)$ & $2.9(5)$ & $2.8(5)$ & $1.2(5)$ \\
\hline C20 & $20.7(6)$ & $21.1(6)$ & $23.4(6)$ & $-0.8(5)$ & $-2.6(5)$ & $-0.4(5)$ \\
\hline C21 & $18.3(6)$ & $20.1(6)$ & $22.2(6)$ & $-1.4(5)$ & $-2.0(4)$ & $1.1(4)$ \\
\hline $\mathrm{H} 21$ & $35(5)$ & $73(10)$ & $34(6)$ & $-26(3)$ & $3(2)$ & $5(4)$ \\
\hline $\mathrm{H} 17$ & $31(5)$ & $61(9)$ & $45(6)$ & $-22(3)$ & $-5(2)$ & $0(4)$ \\
\hline $\mathrm{H} 2 \mathrm{O}$ & $37(5)$ & $69(10)$ & $33(6)$ & $-26(3)$ & $-8(2)$ & $0(4)$ \\
\hline $\mathrm{H} 18$ & $37(5)$ & $55(9)$ & $47(6)$ & $-14(3)$ & $12(3)$ & $10(3)$ \\
\hline H19 & $61(8)$ & 47(9) & $25(2)$ & $-17(5)$ & $0.5(12)$ & $3.1(13)$ \\
\hline $\mathrm{H} 15$ & $27(3)$ & $33(6)$ & $44(8)$ & $-8(2)$ & $12(3)$ & $-12(4)$ \\
\hline $\mathrm{H} 13$ & $65(8)$ & $37(4)$ & $60(9)$ & $-22(3)$ & $6(5)$ & $-11(3)$ \\
\hline $\mathrm{H} 14$ & $59(6)$ & $34(7)$ & $62(9)$ & $-5(3)$ & $26(3)$ & $-16(4)$ \\
\hline H11 & $47(6)$ & $55(8)$ & $77(10)$ & $1(3)$ & $31(4)$ & $-8(4)$ \\
\hline $\mathrm{H} 12$ & $38(4)$ & $60(8)$ & $99(12)$ & $-23(3)$ & $17(3)$ & $-8(5)$ \\
\hline $\mathrm{H} 3 \mathrm{a}$ & $35(3)$ & $38(8)$ & $35(7)$ & $13(2)$ & $-5.7(18)$ & $-5(4)$ \\
\hline $\mathrm{H} 2$ & $52(6)$ & $51(7)$ & $43(8)$ & $-19(3)$ & $-5(4)$ & $-14(4)$ \\
\hline $\mathrm{H} 3 \mathrm{~b}$ & $59(8)$ & $49(7)$ & $33(4)$ & $16(4)$ & $-2(3)$ & $-16(3)$ \\
\hline C4 & $26.8(6)$ & $24.3(6)$ & $18.8(5)$ & $7.8(5)$ & $-3.5(4)$ & $-2.9(4)$ \\
\hline C5 & $35.0(7)$ & $25.6(7)$ & $26.5(7)$ & $-2.2(6)$ & $-8.7(6)$ & $6.8(5)$ \\
\hline H5 & $138(17)$ & $33(4)$ & $79(11)$ & $-18(3)$ & $-69(8)$ & $8(3)$ \\
\hline C6 & $48.6(9)$ & $29.9(7)$ & $32.9(7)$ & $1.8(7)$ & $-15.9(6)$ & $7.3(6)$ \\
\hline H6 & $201(19)$ & $33(4)$ & $135(13)$ & $14(3)$ & $-133(9)$ & $3(3)$ \\
\hline $\mathrm{C7}$ & $33.7(7)$ & $33.8(7)$ & $17.6(5)$ & $5.5(6)$ & $-4.0(5)$ & $-0.0(5)$ \\
\hline $\mathrm{H} 7$ & $56(7)$ & $49(7)$ & $33(5)$ & $5(4)$ & $-23(3)$ & $0(3)$ \\
\hline $\mathrm{C} 8$ & $95.1(14)$ & $31.6(8)$ & 47.7(9) & $11.9(8)$ & $-46.6(9)$ & $-8.4(7)$ \\
\hline H8 & $300(20)$ & $35(4)$ & $165(15)$ & $3(3)$ & $-190(11)$ & $-8(3)$ \\
\hline $\mathrm{C} 9$ & $105.1(16)$ & $27.6(8)$ & $52.6(10)$ & $19.5(10)$ & $-53.1(10)$ & $-11.8(7)$ \\
\hline H9 & $230(20)$ & $32(5)$ & $143(15)$ & $25(4)$ & $-150(11)$ & $-10(3)$ \\
\hline
\end{tabular}

Table S5: Bond Lengths in Å for 11a.

\begin{tabular}{|c|c|c|}
\hline Atom & Atom & Length/Å \\
\hline$\overline{C 1}$ & $\mathrm{C} 2$ & $1.5432(17)$ \\
\hline C1 & C3 & $1.5065(18)$ \\
\hline $\mathrm{C} 1$ & C10 & $1.5063(17)$ \\
\hline $\mathrm{C} 1$ & $\mathrm{C} 4$ & $1.5081(16)$ \\
\hline$C 2$ & $\mathrm{C} 3$ & $1.4956(19)$ \\
\hline C2 & C16 & $1.4862(16)$ \\
\hline C10 & C11 & $1.3938(17)$ \\
\hline C10 & C15 & $1.3938(16)$ \\
\hline C11 & C12 & $1.397(2)$ \\
\hline C12 & C13 & $1.387(2)$ \\
\hline C13 & C14 & $1.3906(19)$ \\
\hline C14 & C15 & $1.3938(17)$ \\
\hline
\end{tabular}

\begin{tabular}{lll}
\hline Atom & Atom & Length/Å \\
\hline C16 & C17 & $1.4031(17)$ \\
C16 & C21 & $1.3990(16)$ \\
C17 & C18 & $1.3865(18)$ \\
C18 & C19 & $1.3881(19)$ \\
C19 & C20 & $1.3911(18)$ \\
C20 & C21 & $1.3862(16)$ \\
C4 & C5 & $1.3832(18)$ \\
C4 & C9 & $1.396(2)$ \\
C5 & C6 & $1.3961(19)$ \\
C6 & C7 & $1.371(2)$ \\
C7 & C8 & $1.371(2)$ \\
C8 & C9 & $1.388(2)$
\end{tabular}


Table S6: Bond Angles in ${ }^{\circ}$ for 11a.

\begin{tabular}{llll}
\hline Atom & Atom & Atom & \multicolumn{1}{c}{ Angle $^{\circ}$} \\
\hline C3 & C1 & C2 & 58.72(9) \\
C10 & C1 & C2 & $118.42(9)$ \\
C10 & C1 & C3 & $118.04(11)$ \\
C4 & C1 & C2 & $116.25(10)$ \\
C4 & C1 & C3 & $117.15(10)$ \\
C4 & C1 & C10 & $116.09(10)$ \\
C3 & C2 & C1 & $59.41(8)$ \\
C16 & C2 & C1 & $124.30(10)$ \\
C16 & C2 & C3 & $122.99(11)$ \\
C2 & C3 & C1 & $61.86(8)$ \\
C11 & C10 & C1 & $120.54(11)$ \\
C15 & C10 & C1 & $120.60(11)$ \\
C15 & C10 & C11 & $118.84(12)$ \\
C12 & C11 & C10 & $120.42(12)$ \\
C13 & C12 & C11 & $120.39(13)$ \\
C14 & C13 & C12 & $119.45(12)$ \\
C15 & C14 & C13 & $120.20(12)$
\end{tabular}

\begin{tabular}{lllc}
\hline Atom & Atom & Atom & Angle/ $^{\circ}$ \\
\hline C14 & C15 & C10 & $120.69(12)$ \\
C17 & C16 & C2 & $118.19(11)$ \\
C21 & C16 & C2 & $123.93(11)$ \\
C21 & C16 & C17 & $117.83(11)$ \\
C18 & C17 & C16 & $121.21(12)$ \\
C19 & C18 & C17 & $120.31(12)$ \\
C20 & C19 & C18 & $119.08(11)$ \\
C21 & C20 & C19 & $120.77(12)$ \\
C20 & C21 & C16 & $120.80(11)$ \\
C5 & C4 & C1 & $121.59(12)$ \\
C9 & C4 & C1 & $121.95(12)$ \\
C9 & C4 & C5 & $116.45(12)$ \\
C6 & C5 & C4 & $121.24(13)$ \\
C7 & C6 & C5 & $121.21(13)$ \\
C8 & C7 & C6 & $118.60(12)$ \\
C9 & C8 & C7 & $120.41(14)$ \\
C8 & C9 & C4 & $122.08(14)$
\end{tabular}

Table S7: Torsion Angles in ${ }^{\circ}$ for 11a.

\begin{tabular}{llllc}
\hline Atom & Atom & Atom & Atom & Angle/ $^{\circ}$ \\
\hline C1 & C2 & C16 & C17 & $-128.57(12)$ \\
C1 & C2 & C16 & C21 & 54.21(14) \\
C1 & C3 & C2 & C16 & $113.37(8)$ \\
C1 & C10 & C11 & C12 & $-178.08(11)$ \\
C1 & C10 & C15 & C14 & $177.95(10)$ \\
C1 & C4 & C5 & C6 & $178.70(6)$ \\
C1 & C4 & C9 & C8 & $-178.68(7)$ \\
C2 & C16 & C17 & C18 & $-176.98(11)$ \\
C2 & C16 & C21 & C20 & $176.71(12)$ \\
C10 & C11 & C12 & C13 & $-0.06(14)$ \\
C10 & C15 & C14 & C13 & $0.31(13)$ \\
C11 & C12 & C13 & C14 & $-0.11(15)$ \\
C12 & C13 & C14 & C15 & $-0.02(14)$ \\
C16 & C17 & C18 & C19 & $0.14(14)$ \\
C16 & C21 & C20 & C19 & $0.08(14)$ \\
C17 & C18 & C19 & C20 & $-0.59(14)$ \\
C18 & C19 & C20 & C21 & $0.48(14)$ \\
C4 & C5 & C6 & C7 & $0.09(11)$ \\
C4 & C9 & C8 & C7 & $-0.12(11)$ \\
C5 & C6 & C7 & C8 & $-0.16(11)$ \\
C6 & C7 & C8 & C9 & $0.18(8)$ \\
& & & &
\end{tabular}


Table S8: Hydrogen Fractional Atomic Coordinates $\left(\times 10^{4}\right)$ and Equivalent Isotropic Displacement Parameters $\left(\AA^{2} \times 10^{3}\right)$ for 11a. $U_{e q}$ is defined as $1 / 3$ of the trace of the orthogonalised $U_{i j}$.

\begin{tabular}{lllll}
\hline Atom & \multicolumn{1}{c}{$\mathbf{x}$} & $\mathbf{y}$ & $\mathbf{z}$ & $\boldsymbol{U}_{\text {eq }}$ \\
\hline H21 & $-10470(20)$ & $-4854(14)$ & $-4701(6)$ & $48(4)$ \\
H17 & $-3920(30)$ & $-3208(13)$ & $-4782(7)$ & $46(4)$ \\
H20 & $-10600(30)$ & $-4854(14)$ & $-5795(6)$ & $46(4)$ \\
H18 & $-4160(30)$ & $-3144(13)$ & $-5898(7)$ & $46(4)$ \\
H19 & $-7560(30)$ & $-3996(13)$ & $-6421(6)$ & $45(4)$ \\
H15 & $-4540(20)$ & $-6272(11)$ & $-4295(6)$ & $35(3)$ \\
H13 & $-9340(30)$ & $-9039(13)$ & $-4462(7)$ & $54(4)$ \\
H14 & $-5570(30)$ & $-8124(12)$ & $-4728(7)$ & $52(4)$ \\
H11 & $-11090(30)$ & $-6191(13)$ & $-3366(8)$ & $59(5)$ \\
H12 & $-12060(30)$ & $-8050(15)$ & $-3784(9)$ & $65(5)$ \\
H3a & $-10540(20)$ & $-4270(11)$ & $-3743(6)$ & $36(3)$ \\
H2 & $-5680(30)$ & $-3388(12)$ & $-3849(7)$ & $49(4)$ \\
H3b & $-9140(30)$ & $-3536(12)$ & $-3182(6)$ & $47(4)$ \\
H5 & $-5440(40)$ & $-6749(13)$ & $-2921(8)$ & $83(7)$ \\
H6 & $-2650(50)$ & $-6769(15)$ & $-2080(10)$ & $123(9)$ \\
H7 & $-1290(30)$ & $-5008(13)$ & $-1631(6)$ & $46(4)$ \\
H8 & $-2530(60)$ & $-3237(16)$ & $-2053(12)$ & $165(11)$ \\
H9 & $-5250(50)$ & $-3212(14)$ & $-2912(10)$ & $135(10)$
\end{tabular}

\section{Citations}

O.V. Dolomanov and L.J. Bourhis and R.J. Gildea and J.A.K. Howard and H. Puschmann, Olex2: A complete structure solution, refinement and analysis program, J. Appl. Cryst., (2009), 42, 339-341. 


\section{Crystal Data and Experimental}

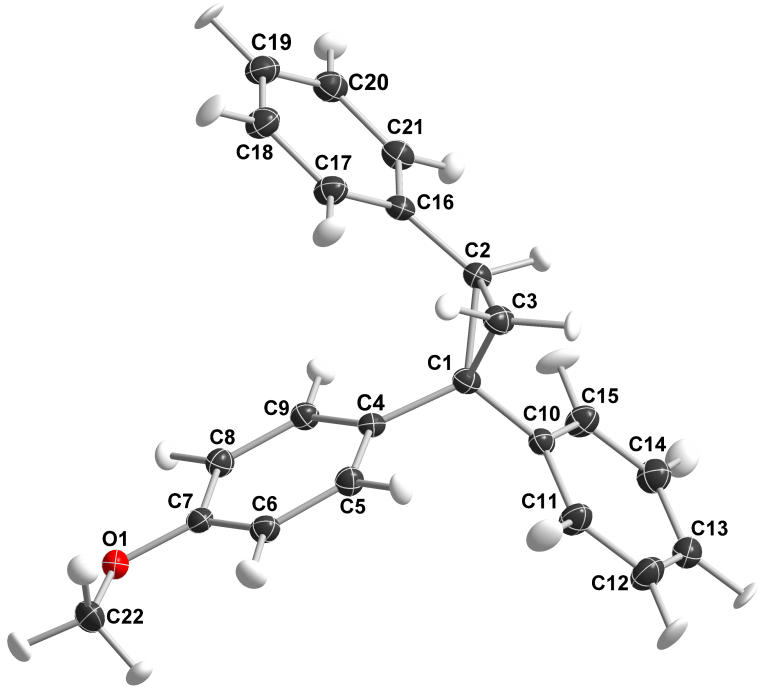

\begin{tabular}{|c|c|}
\hline Compound & $\begin{array}{l}\text { MeOphenylcyclopro } \\
\text { panedibenzene (11c) }\end{array}$ \\
\hline Formula & $\mathrm{C}_{22} \mathrm{H}_{20} \mathrm{O}$ \\
\hline$D_{\text {calc. }} / \mathrm{g} \mathrm{cm}^{-3}$ & 1.232 \\
\hline$\mu / \mathrm{mm}^{-1}$ & 0.567 \\
\hline Formula Weight & 300.403 \\
\hline Color & colorless \\
\hline Shape & prism \\
\hline Size $/ \mathrm{mm}^{3}$ & $0.45 \times 0.28 \times 0.17$ \\
\hline$T / \mathrm{K}$ & $100.3(6)$ \\
\hline Crystal System & monoclinic \\
\hline Flack Parameter & $-0.0(2)$ \\
\hline Hooft Parameter & $0.08(6)$ \\
\hline Space Group & $P 2_{1}$ \\
\hline$a / \AA ̊$ & $11.20707(10)$ \\
\hline$b / \AA$ & $5.82419(4)$ \\
\hline$c / \AA ̊$ & $12.84587(11)$ \\
\hline$\alpha l^{\circ}$ & 90 \\
\hline$\beta 1^{\circ}$ & 104.9956(9) \\
\hline$\left.\gamma\right|^{\circ}$ & 90 \\
\hline$V / \AA^{3}$ & $809.923(12)$ \\
\hline Z & 2 \\
\hline$Z^{\prime}$ & 1 \\
\hline Wavelength/Å & 1.54184 \\
\hline Radiation type & $\mathrm{Cu} \mathrm{K} \alpha$ \\
\hline$\Theta_{\min } /^{\circ}$ & 3.56 \\
\hline$\Theta_{\max } I^{\circ}$ & 76.99 \\
\hline Measured Refl's. & 24510 \\
\hline Ind't Refl's & 3230 \\
\hline Refl's with $I \geq \sigma(I)$ & 3163 \\
\hline$R_{\text {int }}$ & 0.0396 \\
\hline Parameters & 130 \\
\hline Restraints & 1 \\
\hline Largest Peak & 0.1068 \\
\hline Deepest Hole & -0.1396 \\
\hline GooF & 1.0592 \\
\hline$w R_{2}$ (all data) & 0.0554 \\
\hline$w R_{2}$ & 0.0551 \\
\hline$R_{1}$ (all data) & 0.0220 \\
\hline$R_{1}$ & 0.0214 \\
\hline
\end{tabular}


Structure Quality Indicators

Reflections:

$\mathrm{d} \min (\mathrm{Cu})$

0.79

$57.3^{\text {Rint }}$

$3.96 \%$ fomplete $100 \%$

Refinement:

Shift 0.001

Max Peak 0.1

Min Peak $\quad-0.1$

GooF 1.059

Flack $\quad-.0(2)$

A colourless prism-shaped crystal with dimensions $0.45 \times 0.28 \times 0.17 \mathrm{~mm}^{3}$ was mounted on a loop. Data were collected using a XtaLAB Synergy, Dualflex, HyPix diffractometer equipped with an Oxford Cryosystems lowtemperature device operating at $T=100.3(6) \mathrm{K}$.

Data were measured using $\omega$ scans using $\mathrm{Cu} \mathrm{K}_{\alpha}$ radiation. The diffraction pattern was indexed and the total number of runs and images was based on the strategy calculation from the program CrysAlisPro 1.171.40.68a (Rigaku OD, 2019). The maximum resolution that was achieved was $\Theta=76.99^{\circ}(0.79 \AA)$. The unit cell was refined using CrysAlisPro 1.171.40.68a (Rigaku OD, 2019) on 20958 reflections, $86 \%$ of the observed reflections.

Data reduction, scaling and absorption corrections were performed using CrysAlisPro 1.171.40.68a (Rigaku OD, 2019). The final completeness is $99.81 \%$ out to $76.99^{\circ}$ in $\Theta$. A numerical absorption correction based on Gaussian integration over a multifaceted crystal model was performed using CrysAlisPro 1.171.40.68a (Rigaku Oxford Diffraction, 2019). An empirical absorption correction using spherical harmonics, implemented in SCALE3 ABSPACK scaling algorithm was also applied. The absorption coefficient $\mu$ of this material is $0.567 \mathrm{~mm}^{-1}$ at this wavelength $(\lambda=1.54184 \AA)$ and the minimum and maximum transmissions are 0.420 and 1.000 .

The structure was solved and the space group $P 2_{1}(\# 4)$ determined by the ShelXT structure solution program using dual methods and refined by full matrix least squares minimisation on $\boldsymbol{F}^{\mathbf{2}}$ using version of olex2.refine 1.3 -alpha. ${ }^{19}$ All non-hydrogen atoms were refined anisotropically. Hydrogen atom positions were calculated geometrically and refined using the riding model.

_refine_special_details: Refinement using NoSpherA2, an implementation of NOn-SPHERical Atom-form-factors in Olex2. ${ }^{20}$ NoSpherA2 makes use of tailor-made aspherical atomic form factors calculatedon-the-fly from a Hirshfeldpartitioned electron density (ED) - not fromspherical-atom form factors. The ED is calculated from a gaussian basis set single determinant SCFwavefunction - either Hartree-Fock or B3LYP - for a fragment of the crystal embedded inan electrostatic crystal field.The following options were used: SOFTWARE: Tonto METHOD: rks BASIS SET: def2SVP CHARGE: 0 MULTIPLICITY: 1 DATE: 2020-01-21_17-15-31 CLUSTER RADIUS: 0

There is a single molecule in the asymmetric unit, which is represented by the reported sum formula. In other words: $Z$ is 2 and $Z^{\prime}$ is 1.

The Flack parameter was refined to -0.0(2). Determination of absolute structure using Bayesian statistics on Bijvoet differences using the Olex2 results in 0.08(6). Note: The Flack parameter is used to determine chirality of the crystal studied, the value should be near 0 , a value of 1 means that the stereochemistry is wrong and the model should be inverted. A value of 0.5 means that the crystal consists of a racemic mixture of the two enantiomers. 


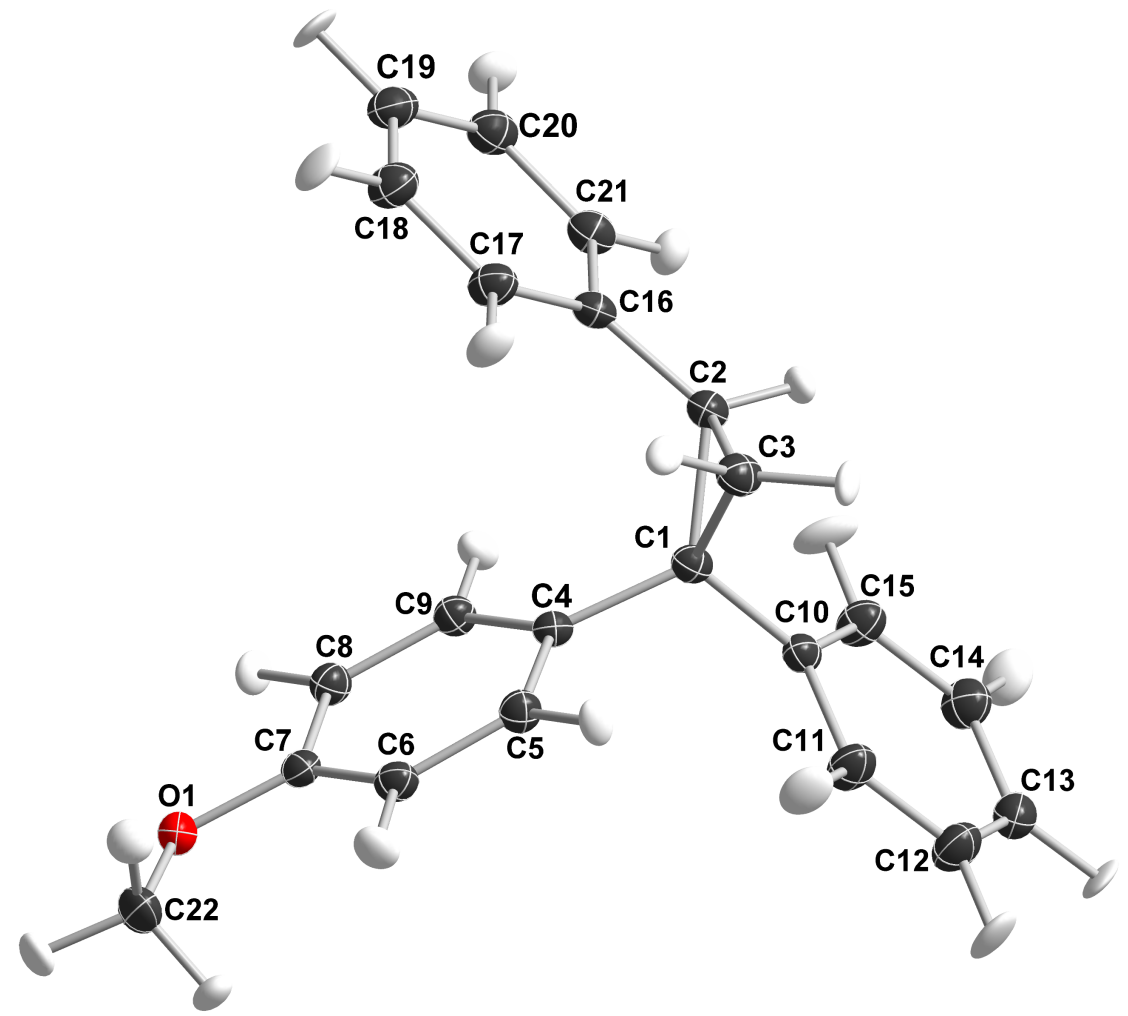

Figure S2: Thermal ellipsoid plot of the molecular structure.

\section{Data Plots: Diffraction Data}
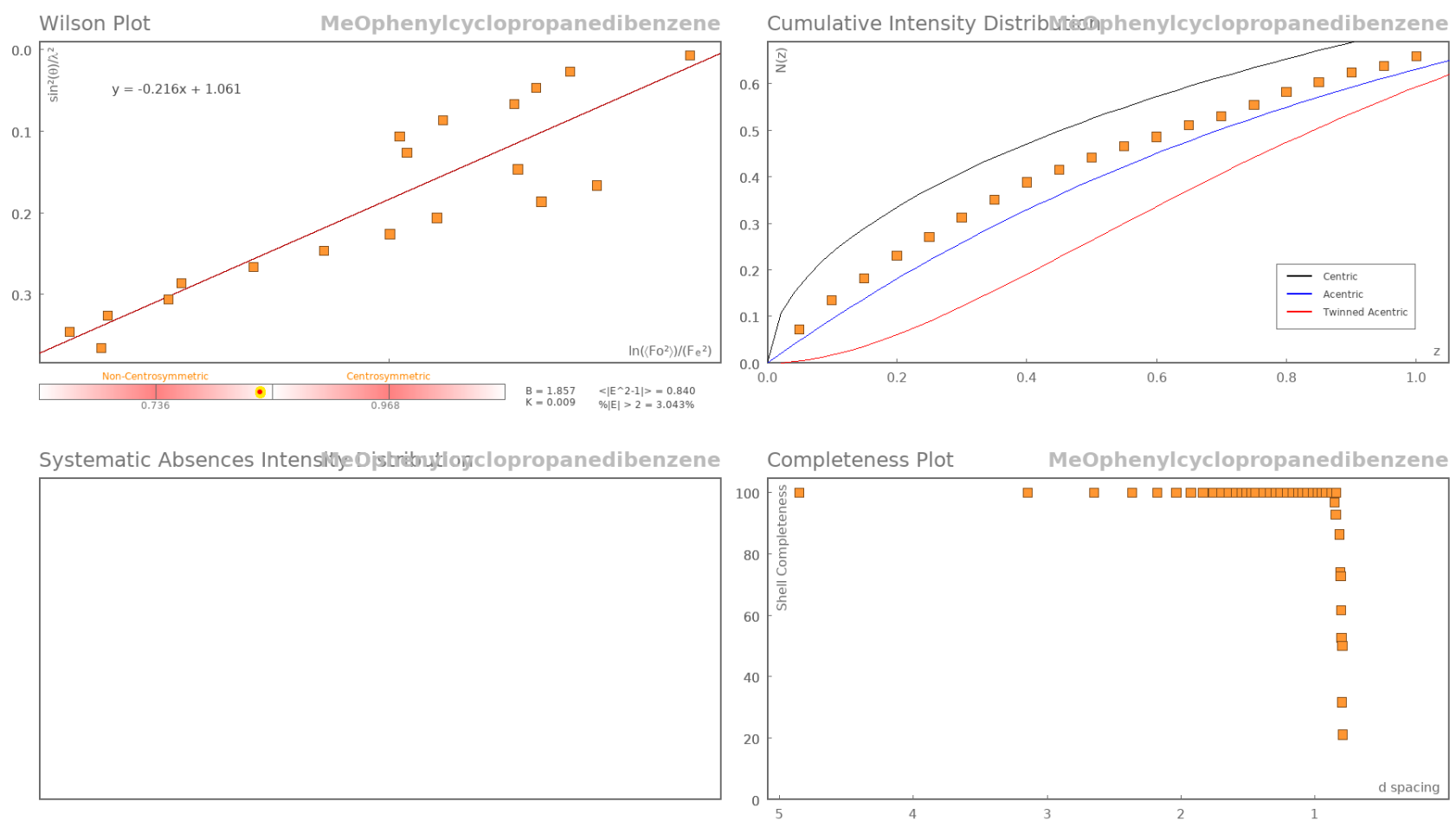


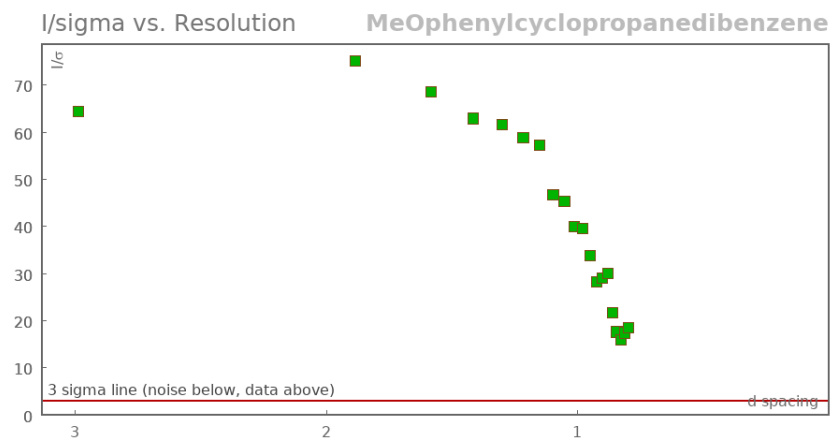

Data Plots: Refinement and Data
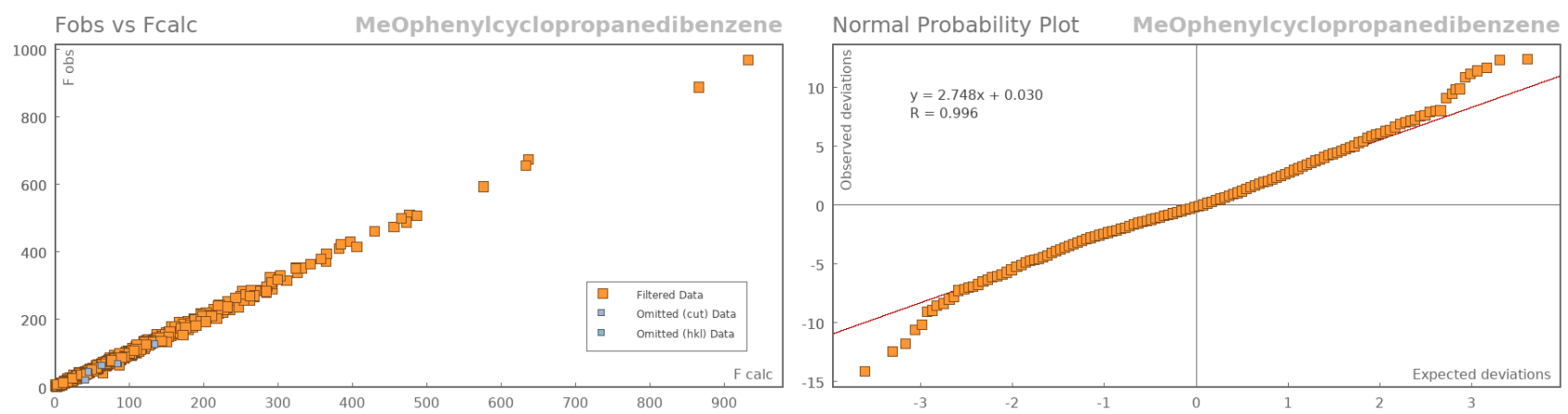

\section{Reflection Statistics}

Total reflections (after filtering) 24510

Completeness

$\mathrm{hkI}_{\max }$ collected

$\mathrm{hkl}_{\text {max }}$ used

Lim $d_{\text {max }}$ collected

$\mathrm{d}_{\text {max }}$ used

Friedel pairs

Inconsistent equivalents

$\mathrm{R}_{\text {sigma }}$

Omitted reflections

Multiplicity

Removed systematic absences

\subsection{4}

$(13,7,15)$

$(13,7,16)$

100.0

12.41

2724

9

0.0174

0

$394,251,162,71,21,11,5,2,1)$
Unique reflections

Mean $\mathrm{I} / \sigma$

$\mathrm{hk} \mathrm{I}_{\text {min }}$ collected

$\mathrm{hkl}_{\min }$ used

Lim $d_{\text {min }}$ collected

$d_{\text {min }}$ used

Friedel pairs merged

$R_{\text {int }}$

Intensity transformed

Omitted by user (OMIT hkl)

Maximum multiplicity

Filtered off (Shel/OMIT)
3231

43.07

$(-12,-7,-16)$

$(-13,-7,0)$

0.77

0.79

0

0.0396

0

13

20

0

\section{Images of the Crystal on the Diffractometer}
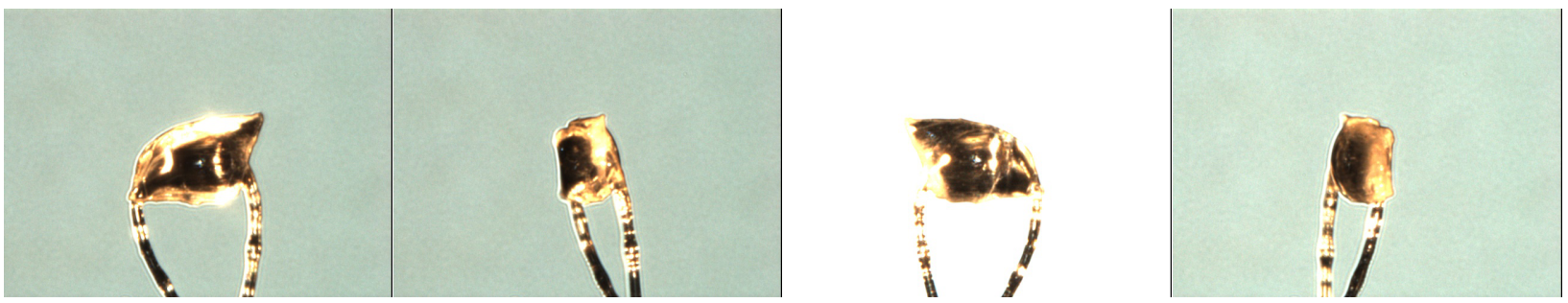

Table S9: Fractional Atomic Coordinates $\left(\times 10^{4}\right)$ and Equivalent Isotropic Displacement Parameters $\left(\AA^{2} \times 10^{3}\right)$ for 11 c. $U_{\text {eq }}$ is defined as $1 / 3$ of the trace of the orthogonalised $U_{i j}$. 


\begin{tabular}{lrrrl}
\hline Atom & \multicolumn{1}{c}{$\mathbf{x}$} & $\mathbf{y}$ & $\mathbf{z}$ & $\boldsymbol{U}_{\text {eq }}$ \\
\hline O1 & $3207.5(5)$ & $4599.3(10)$ & $536.0(4)$ & 21.461 \\
C7 & $4380.9(7)$ & $4371.1(14)$ & $1160.4(6)$ & 17.203 \\
C2 & $8489.5(7)$ & $4538.1(14)$ & $4352.0(6)$ & 18.596 \\
C16 & $7621.4(7)$ & $5109.8(14)$ & $5018.8(6)$ & 17.93 \\
C22 & $2673.1(8)$ & $2676.3(17)$ & $-95.6(7)$ & 27.244 \\
C1 & $8135.8(7)$ & $4197.8(13)$ & $3132.3(6)$ & 16.139 \\
C5 & $6320.9(7)$ & $2408.1(14)$ & $1819.2(6)$ & 18.029 \\
C17 & $6666.7(8)$ & $3651.9(15)$ & $5115.9(7)$ & 22.343 \\
C8 & $4848.1(8)$ & $6214.2(14)$ & $1846.0(6)$ & 18.708 \\
C10 & $9057.5(7)$ & $5033.7(14)$ & $2542.7(6)$ & 16.757 \\
C4 & $6810.7(7)$ & $4244.0(13)$ & $2492.9(6)$ & 14.952 \\
C3 & $8565.4(8)$ & $2192.5(15)$ & $3876.7(6)$ & 20.701 \\
C18 & $5922.0(8)$ & $4197.7(16)$ & $5795.2(7)$ & 24.791 \\
C21 & $7799.0(8)$ & $7151.8(14)$ & $5618.5(6)$ & 20.452 \\
C15 & $9729.5(8)$ & $7047.5(14)$ & $2831.0(7)$ & 22.406 \\
C13 & $10841.8(8)$ & $6423.9(16)$ & $1481.5(7)$ & 24.409 \\
C14 & $10611.1(8)$ & $7748.1(16)$ & $2305.9(7)$ & 25.268 \\
C6 & $5122.6(7)$ & $2455.6(14)$ & $1151.2(6)$ & 18.897 \\
C9 & $6045.7(7)$ & $6144.3(14)$ & $2496.7(6)$ & 17.263 \\
C11 & $9291.6(8)$ & $3725.7(16)$ & $1702.9(7)$ & 24.33 \\
C19 & $6120.1(8)$ & $6221.5(16)$ & $6397.7(7)$ & 24.632 \\
C12 & $10173.1(8)$ & $4402.1(17)$ & $1182.4(7)$ & 27.453 \\
C20 & $7061.4(8)$ & $7696.9(15)$ & $6302.8(7)$ & 23.491 \\
& & & &
\end{tabular}

Table S10: Anisotropic Displacement Parameters $\left(\times 10^{4}\right)$ for 11c. The anisotropic displacement factor exponent takes the form: $-2 \pi^{2}\left[h^{2} a^{* 2} \times U_{11}+\ldots+2 h k a^{*} \times b^{*} \times U_{12}\right]$

\begin{tabular}{|c|c|c|c|c|c|c|}
\hline Atom & $U_{11}$ & $U_{22}$ & $U_{33}$ & $U_{23}$ & $U_{13}$ & $U_{12}$ \\
\hline$\overline{01}$ & 16.07 & 29.39 & 19.08 & 1.59 & 4.83 & -0.65 \\
\hline $\mathrm{C} 7$ & 16.59 & 21.36 & 14.76 & -1.53 & 6.04 & -0.02 \\
\hline $\mathrm{C} 2$ & 17.74 & 22.08 & 15 & 0.95 & 2.49 & 0.28 \\
\hline C16 & 19.27 & 19.79 & 13.25 & 0.16 & 1.54 & 0.01 \\
\hline $\mathrm{C} 22$ & 18.23 & 39.55 & 22.05 & -4 & 1.78 & -6.25 \\
\hline $\mathrm{C} 1$ & 18.03 & 14.81 & 15.06 & 1.78 & 3.35 & -1.04 \\
\hline C5 & 18.72 & 16.39 & 19.28 & 0.39 & 5.46 & -2.83 \\
\hline C17 & 24.91 & 22.97 & 19.93 & -3.64 & 7.21 & -2.87 \\
\hline C8 & 18.83 & 19.25 & 18.35 & 1.87 & 5.36 & -0.3 \\
\hline C10 & 15.82 & 18.21 & 15.59 & 2.22 & 2.89 & -0.37 \\
\hline C4 & 16.72 & 15.14 & 13.14 & 0.05 & 4.12 & -0.3 \\
\hline C3 & 22.51 & 20.39 & 18.5 & 3.96 & 4.04 & 2.42 \\
\hline C18 & 26.83 & 26.94 & 22.83 & -2.98 & 10.44 & -0.79 \\
\hline C21 & 22.57 & 17.99 & 19.27 & -0.09 & 2.66 & -0.13 \\
\hline C15 & 25.91 & 19.84 & 23.28 & -2.68 & 9.63 & -4.53 \\
\hline C13 & 19.74 & 31.16 & 23.01 & 1.45 & 6.76 & 2.18 \\
\hline C14 & 25.26 & 25.08 & 26.4 & -4.85 & 8.37 & -0.68 \\
\hline C6 & 19.81 & 20.2 & 17.11 & -2.43 & 5.55 & -3.22 \\
\hline C9 & 18.61 & 16.11 & 17.01 & 0.4 & 4.5 & -2.3 \\
\hline C11 & 25.76 & 25.95 & 23.31 & -2.35 & 10.01 & -7.75 \\
\hline C19 & 27.97 & 28.1 & 18.9 & 3.18 & 8 & -0.26 \\
\hline C12 & 27.25 & 32.13 & 26.41 & -0.12 & 13.13 & -6.09 \\
\hline C20 & 26.61 & 22.12 & 20.74 & 3.29 & 4.32 & -2.63 \\
\hline $\mathrm{H} 2 \mathrm{O}$ & 58.92 & 35.5 & 45.4 & -1.42 & 19.21 & -16.9 \\
\hline H9 & 41.15 & 45.69 & 35.75 & 10.43 & 2.36 & -10.21 \\
\hline
\end{tabular}




\begin{tabular}{llllccc}
\hline Atom & \multicolumn{1}{c}{$\boldsymbol{U}_{\mathbf{1 1}}$} & $\boldsymbol{U}_{\mathbf{2 2}}$ & $\boldsymbol{U}_{\mathbf{3 3}}$ & $\boldsymbol{U}_{\mathbf{2 3}}$ & $\boldsymbol{U}_{\mathbf{1 3}}$ & $\boldsymbol{U}_{\mathbf{1 2}}$ \\
\hline $\mathrm{H} 5$ & 28.25 & 23.49 & 50.11 & 7.63 & 2.06 & -17.74 \\
H21a & 38.85 & 69.42 & 34.82 & -11.06 & 22.09 & -17.57 \\
H8 & 34.19 & 19.01 & 48.06 & 8.97 & 11.41 & -3.76 \\
H18 & 51.84 & 39.29 & 61.83 & -24.63 & 42.67 & -8.04 \\
H2 & 21.67 & 48.19 & 32.81 & -5.82 & 1.88 & -4.79 \\
H21b & 25.14 & 67.18 & 41.9 & -5.15 & -6.01 & -11.67 \\
H6 & 47.3 & 37.85 & 42.35 & -7.44 & 2.77 & -13.64 \\
H13 & 38.04 & 65.47 & 42.66 & -2.62 & 35.78 & 6.96 \\
H12 & 57.14 & 67.03 & 62.66 & -18.12 & 47.5 & -39.3 \\
H11 & 71.37 & 34.04 & 60.22 & -18.52 & 31.7 & -27.12 \\
H15 & 101.38 & 39.9 & 44.43 & -30.28 & 44.9 & -23.9 \\
H3a & 37.02 & 29 & 38.68 & 6.15 & 13.9 & 14.35 \\
H3b & 20 & 39.07 & 49.91 & 13.11 & 13.82 & 8.71 \\
H14 & 63.5 & 35.12 & 81.64 & -16.27 & 32.26 & -6.81 \\
H19 & 53.08 & 75.8 & 43.52 & 0.16 & 41.01 & -18.5 \\
H00E & 38.79 & 40.56 & 54.79 & -5.57 & 21.67 & -9.91 \\
H17 & 57.71 & 42.25 & 53.52 & -12.8 & 30.51 & -23.81 \\
H21c & 50.51 & 40.8 & 48.21 & -11.26 & 12.04 & -2.59
\end{tabular}

Table S11: Bond Lengths in Å for 11c.

\begin{tabular}{lll}
\hline Atom & Atom & Length/A \\
\hline O1 & C7 & $1.3581(9)$ \\
O1 & C22 & $1.4212(11)$ \\
C7 & C8 & $1.4012(11)$ \\
C7 & C6 & $1.3931(11)$ \\
C2 & C16 & $1.4907(11)$ \\
C2 & C1 & $1.5264(10)$ \\
C2 & C3 & $1.5077(12)$ \\
C16 & C17 & $1.3965(12)$ \\
C16 & C21 & $1.4029(11)$ \\
C1 & C10 & $1.5108(11)$ \\
C1 & C4 & $1.4998(10)$ \\
C1 & C3 & $1.5073(11)$ \\
C5 & C4 & $1.3956(11)$
\end{tabular}

\begin{tabular}{lll}
\hline Atom & Atom & Length/A \\
\hline C5 & C6 & $1.3943(11)$ \\
C17 & C18 & $1.3914(12)$ \\
C8 & C9 & $1.3861(11)$ \\
C10 & C15 & $1.3913(11)$ \\
C10 & C11 & $1.3997(11)$ \\
C4 & C9 & $1.4008(10)$ \\
C18 & C19 & $1.3959(13)$ \\
C21 & C20 & $1.3904(12)$ \\
C15 & C14 & $1.3937(12)$ \\
C13 & C14 & $1.3875(13)$ \\
C13 & C12 & $1.3953(13)$ \\
C11 & C12 & $1.3857(12)$ \\
C19 & C20 & $1.3902(13)$
\end{tabular}

Table S12: Bond Angles in for 11c.

\begin{tabular}{llll}
\hline Atom & Atom & Atom & Angle/ \\
\hline C22 & O1 & C7 & $117.42(6)$ \\
C8 & C7 & O1 & $116.32(7)$ \\
C6 & C7 & O1 & $124.20(7)$ \\
C6 & C7 & C8 & $119.48(7)$ \\
C1 & C2 & C16 & $125.73(6)$ \\
C3 & C2 & C16 & $123.27(7)$ \\
C3 & C2 & C1 & $59.57(5)$ \\
C17 & C16 & C2 & $122.99(7)$ \\
C21 & C16 & C2 & $118.94(7)$ \\
C21 & C16 & C17 & $118.00(7)$ \\
C10 & C1 & C2 & $116.30(6)$ \\
C4 & C1 & C2 & $121.14(6)$ \\
C4 & C1 & C10 & $115.35(6)$
\end{tabular}

\begin{tabular}{llll}
\hline Atom & Atom & Atom & \multicolumn{1}{c}{ Angle $^{\circ}$} \\
\hline C3 & C1 & C2 & $59.60(5)$ \\
C3 & C1 & C10 & $114.84(6)$ \\
C3 & C1 & C4 & $118.02(7)$ \\
C6 & C5 & C4 & $121.88(7)$ \\
C18 & C17 & C16 & $120.99(8)$ \\
C9 & C8 & C7 & $120.17(7)$ \\
C15 & C10 & C1 & $122.10(7)$ \\
C11 & C10 & C1 & $119.98(7)$ \\
C11 & C10 & C15 & $117.87(8)$ \\
C5 & C4 & C1 & $120.05(7)$ \\
C9 & C4 & C1 & $122.17(7)$ \\
C9 & C4 & C5 & $117.68(7)$ \\
C1 & C3 & C2 & $60.83(5)$
\end{tabular}




\begin{tabular}{lllr}
\hline Atom & Atom & Atom & Angle/ $^{\circ}$ \\
\hline C19 & C18 & C17 & $120.36(8)$ \\
C20 & C21 & C16 & $121.18(8)$ \\
C14 & C15 & C10 & $121.38(8)$ \\
C12 & C13 & C14 & $119.10(8)$ \\
C13 & C14 & C15 & $120.14(8)$ \\
C5 & C6 & C7 & $119.50(7)$
\end{tabular}

\begin{tabular}{llll}
\hline Atom & Atom & Atom & Angle/ $^{\circ}$ \\
\hline C4 & C9 & C8 & $121.28(7)$ \\
C12 & C11 & C10 & $121.09(8)$ \\
C20 & C19 & C18 & $119.26(8)$ \\
C11 & C12 & C13 & $120.41(8)$ \\
C19 & C20 & C21 & $120.20(8)$
\end{tabular}

Table S13: Torsion Angles in ${ }^{\circ}$ for 11c.

\begin{tabular}{llllr}
\hline Atom & Atom & Atom & Atom & \multicolumn{1}{c}{ Angle/ } \\
\hline O1 & C7 & C8 & C9 & $-178.80(6)$ \\
O1 & C7 & C6 & C5 & $179.39(7)$ \\
C7 & C8 & C9 & C4 & $-0.45(9)$ \\
C7 & C6 & C5 & C4 & $-0.79(9)$ \\
C2 & C16 & C17 & C18 & $176.35(8)$ \\
C2 & C16 & C21 & C20 & $-176.10(7)$ \\
C2 & C1 & C10 & C15 & $-38.16(8)$ \\
C2 & C1 & C10 & C11 & $139.40(7)$ \\
C2 & C1 & C4 & C5 & $-125.77(8)$ \\
C2 & C1 & C4 & C9 & $57.90(9)$ \\
C2 & C3 & C1 & C10 & $107.13(6)$ \\
C2 & C3 & C1 & C4 & $-111.53(5)$ \\
C16 & C17 & C18 & C19 & $-0.20(10)$ \\
C16 & C21 & C20 & C19 & $-0.55(9)$ \\
C1 & C10 & C15 & C14 & $177.46(8)$ \\
C1 & C10 & C11 & C12 & $-177.03(8)$ \\
C1 & C4 & C5 & C6 & $-175.16(7)$ \\
C1 & C4 & C9 & C8 & $175.71(7)$ \\
C5 & C4 & C9 & C8 & $-0.71(8)$ \\
C17 & C18 & C19 & C20 & $0.66(10)$ \\
C10 & C15 & C14 & C13 & $-0.44(10)$ \\
C10 & C11 & C12 & C13 & $-0.53(10)$ \\
C18 & C19 & C20 & C21 & $-0.29(10)$ \\
C15 & C14 & C13 & C12 & $0.55(10)$
\end{tabular}


Table S14: Hydrogen Fractional Atomic Coordinates $\left(\times 10^{4}\right)$ and Equivalent Isotropic Displacement Parameters $\left(\AA^{2} \times 10^{3}\right)$ for 11c. $U_{e q}$ is defined as $1 / 3$ of the trace of the orthogonalised $U_{i j}$.

\begin{tabular}{llrll}
\hline Atom & \multicolumn{1}{c}{$\mathbf{x}$} & $\mathbf{y}$ & $\mathbf{z}$ & \multicolumn{1}{c}{$\boldsymbol{U}_{\text {eq }}$} \\
\hline H20 & $7218(11)$ & $9290(20)$ & $6766(9)$ & 45.55 \\
H9 & $6409(11)$ & $7590(20)$ & $3029(9)$ & 42.266 \\
H5 & $6904(10)$ & $873(19)$ & $1808(9)$ & 35.443 \\
H21a & $3199(11)$ & $2220(30)$ & $-672(9)$ & 45.375 \\
H8 & $4248(10)$ & $7725(18)$ & $1858(9)$ & 33.611 \\
H18 & $5202(12)$ & $2960(20)$ & $5867(11)$ & 45.817 \\
H2 & $9389(9)$ & $5410(20)$ & $4632(8)$ & 35.179 \\
H21b & $1718(10)$ & $3170(20)$ & $-543(9)$ & 47.455 \\
H6 & $4786(11)$ & $930(20)$ & $621(9)$ & 44.132 \\
H13 & $11555(10)$ & $6880(30)$ & $1070(9)$ & 44.039 \\
H12 & $10339(12)$ & $3310(30)$ & $534(10)$ & 56.36 \\
H11 & $8774(12)$ & $2100(20)$ & $1480(10)$ & 52.497 \\
H15 & $9576(14)$ & $8110(20)$ & $3486(10)$ & 57.09 \\
H3a & $7857(10)$ & $955(19)$ & $3957(9)$ & 34.141 \\
H3b & $9490(10)$ & $1460(20)$ & $3916(10)$ & 35.444 \\
H14 & $11097(12)$ & $9340(20)$ & $2550(11)$ & 57.594 \\
H19 & $5540(11)$ & $6660(30)$ & $6942(9)$ & 52.195 \\
H00E & $8541(11)$ & $8370(20)$ & $5542(10)$ & 42.945 \\
H17 & $6482(12)$ & $1980(20)$ & $4648(10)$ & 48.18 \\
H21c & $2596(12)$ & $1230(20)$ & $429(10)$ & 46.642 \\
& & & &
\end{tabular}




\section{References}

(1) Davies, H. M.; Nagashima, T.; Klino, J. L., 3rd Stereoselectivity of methyl aryldiazoacetate cyclopropanations of 1,1-diarylethylene. Asymmetric synthesis of a cyclopropyl analogue of tamoxifen. Org. Lett. 2000, 2, 823.

(2) Mahdi, J.; Ankati, H.; Gregory, J.; Tenner, B.; Biehl, E. R. Synthesis of (2-chlorophenyl)(phenyl)methanones and 2-(2-chlorophenyl)1-phenylethanones by Friedel-Crafts acylation of 2-chlorobenzoic acids and 2-(2-chlorophenyl)acetic acids using microwave heating. Tetrahedron Lett. 2011, 52, 2594.

(3) (a) Li, H.; Xu, Y.; Shi, E.; Wei, W.; Suo, X.; Wan, X. Synthesis of arylketones by ruthenium-catalyzed cross-coupling of aldehydes with arylboronic acids. Chem. Commun. 2011, 47, 7880. (b) Yu, Z.; van Veldhoven, J. P.; t Hart, I. M.; Kopf, A. H.; Heitman, L. H.; AP, I. J. Synthesis and biological evaluation of negative allosteric modulators of the Kv11.1(hERG) channel. Eur.J. Med. Chem. 2015, 106, 50.

(4) Schmitt, E.; Landelle, G.; Vors, J.-P.; Lui, N.; Pazenok, S.; Leroux, F. R. A General Approach towards NH-Pyr-azoles That Bear Diverse Fluoroalkyl Groups by Means of Fluorinated Iminium Salts. Eur. J. Org. Chem. 2015, 2015, 6052.

(5) H Harry Szmant, C. M. H. Hydrazones of Nitrophenyl and Pyridyl Aldehydes and ketones. J. Am. Chem. Soc. 1958, 81, 962.

(6) Uchida, H. S. a. T. Monochromatic Light-induced reactions of Benzophenone Hydrazones and the N-Acetyl Derivatives in Carbon Tetrachloride in the Presence of Oxygen. Bull. Chem. Soc.Jpn. 1989, 53, 3225.

(7) Shaffer, M. W.; Leyva, E.; Soundararajan, N.; Chang, E.; Chang, D. H. S.; Capuano, V.; Platz, M. S. Contributions of quantum mechanical tunneling to the rate of benzylic hydrogen atom abstraction reactions of triplet diarylcarbenes in fluid solution. The Journal of Physical Chemistry 1991, 95, 7273.

(8) Nicolle, S. M.; Moody, C. J. Potassium N-iodo p-toluenesulfonamide (TsNIK, Iodamine-T): a new reagent for the oxidation of hydrazones to diazo compounds. Chemistry 2014, 20, 4420.

(9) Humphreys, R. W. R.; Arnold, D. R. Substituent effects on the zero-field splitting parameters of diarylmethylene. Evidence for merostabilization in appropriately substituted diphenylmethylenes. Can. J. Chem. 1979, 57, 2652.

(10) Liu, H.; Wei, Y.; Cai, C. Hypervalent-iodine(iii) oxidation of hydrazones to diazo compounds and one-pot nickel(ii)-catalyzed cyclopropanation. NewJ. Chem. 2016, 40, 674.

(11) [3+2] Cycloaddition of cyclopropane with vinyl ether via photoinduced electron transfer. Tetrahedron Lett. 1989, 30, 4685.

(12) Hideo, T.; Osamu, I. Effects of Aryl Substituents on Electron-Transfer-Mediated Photochemical Addition of Alcohol to 1,1,2Triarylcyclopropanes. Bull. Chem. Soc.Jpn. 1988, 61, 1404.

(13) Gaussian 09, Revision E.01, Frisch, M. J.; Trucks, G. W.; Schlegel, H. B.; Scuseria, G. E.; Robb, M. A.; Cheeseman, J. R.; Scalmani, G.; Barone, V.; Mennucci, B.; Petersson, G. A.; Nakatsuji, H.; Caricato, M.; Li, X.; Hratchian, H. P.; Izmaylov, A. F.; Bloino, J.; Zheng, G.; Sonnenberg, J. L.; Hada, M.; Ehara, M.; Toyota, K.; Fukuda, R.; Hasegawa, J.; Ishida, M.; Nakajima, T.; Honda, Y.; Kitao, O.; Nakai, H.; Vreven, T.; Montgomery, J. A., Jr.; Peralta, J. E.; Ogliaro, F.; Bearpark, M.; Heyd, J. J.; Brothers, E.; Kudin, K. N.; Staroverov, V. N.; Kobayashi, R.; Normand, J.; Raghavachari, K.; Rendell, A.; Burant, J. C.; Iyengar, S. S.; Tomasi, J.; Cossi, M.; Rega, N.; Millam, M. J.; Klene, M.; Knox, J. E.; Cross, J. B.; Bakken, V.; Adamo, C.; Jaramillo, J.; Gomperts, R.; Stratmann, R. E.; Yazyev, O.; Austin, A. J.; Cammi, R.; Pomelli, C.; Ochterski, J. W.; Martin, R. L.; Morokuma, K.; Zakrzewski, V. G.; Voth, G. A.; Salvador, P.; Dannenberg, J. J.; Dapprich, S.; Daniels, A. D.; Farkas, Ö.; Foresman, J. B.; Ortiz, J. V.; Cioslowski, J.; Fox, D. J. Gaussian, Inc., Wallingford CT, 2009.

(14) (a) Becke, A. D. Density-functional exchange-energy approximation with correct asymptotic behavior. Phys. Rev. A 1988, 38, 3098. (b) Lee, C.; Yang, W.; Parr, R. G. Development of the Colle-Salvetti correlation-energy formula into a functional of the electron density. Phys. Rev. $B$ 1988, 37, 785. (c) Becke, A. D. A new mixing of Hartree-Fock and local density-functional theories. J. Chem. Phys. 1993, $98,1372$.

(15) Grimme, S.; Antony, J.; Ehrlich, S.; Krieg, H. A consistent and accurate ab initio parametrization of density functional dispersion correction (DFT-D) for the 94 elements H-Pu. J. Chem. Phys. 2010, 132, 154104.

(16) (a) Hay, P. J.; Wadt, W. R. Ab initio effective core potentials for molecular calculations. Potentials for the transition metal atoms $\mathrm{Sc}$ to $\mathrm{Hg}$. J. Chem. Phys. 1985, 82, 270. (b) Hay, P.J.; Wadt, W. R. Ab initio effective core potentials for molecular calculations. Potentials for K to Au including the outermost core orbitals. J. Chem. Phys. 1985, 82, 299. (c) Wadt, W. R.; Hay, P. J. Ab initio effective core potentials for molecular calculations. Potentials for main group elements Na to Bi. J. Chem. Phys. 1985, 82, 284.

(17) (a) Barone, V.; Cossi, M. Quantum Calculation of Molecular Energies and Energy Gradients in Solution by a Conductor Solvent Model. J. Phys. Chem. A 1998, 102, 1995. (b) Cossi, M.; Rega, N.; Scalmani, G.; Barone, V. Energies, structures, and electronic properties of molecules in solution with the C-PCM solvation model. J. Comput. Chem. 2003, 24, 669.

(18) Sheldrick, G. SHELXT - Integrated space-group and crystal-structure determination. Acta Crystallographica Section A 2015, 71, 3.

(19) Bourhis, L. J.; Dolomanov, O. V.; Gildea, R. J.; Howard, J. A. K.; Puschmann, H. The anatomy of a comprehensive constrained, restrained refinement program for the modern computing environment - Olex2 dissected. Acta Crystallographica Section A 2015, 71, 59.

(20) Dolomanov, O. V.; Bourhis, L. J.; Gildea, R. J.; Howard, J. A. K.; Puschmann, H. OLEX2: a complete structure solution, refinement and analysis program. J. Appl. Crystallogr. 2009, 42, 339. 